\title{
WestVirginiaUniversity
}

THE RESEARCH REPOSITORY @ WVU

Graduate Theses, Dissertations, and Problem Reports

2018

\section{The Bobcats of West Virginia: How Many and Where Are They?}

Thomas F Rounsville Jr

Follow this and additional works at: https://researchrepository.wvu.edu/etd

\section{Recommended Citation}

Rounsville, Thomas F Jr, "The Bobcats of West Virginia: How Many and Where Are They?" (2018).

Graduate Theses, Dissertations, and Problem Reports. 7292.

https://researchrepository.wvu.edu/etd/7292

This Dissertation is protected by copyright and/or related rights. It has been brought to you by the The Research Repository @ WVU with permission from the rights-holder(s). You are free to use this Dissertation in any way that is permitted by the copyright and related rights legislation that applies to your use. For other uses you must obtain permission from the rights-holder(s) directly, unless additional rights are indicated by a Creative Commons license in the record and/ or on the work itself. This Dissertation has been accepted for inclusion in WVU Graduate Theses, Dissertations, and Problem Reports collection by an authorized administrator of The Research Repository @ WVU.

For more information, please contact researchrepository@mail.wvu.edu. 


\title{
The Bobcats of West Virginia: How Many and Where Are They?
}

Thomas F. Rounsville Jr.

Dissertation submitted

to The Davis College of Agriculture, Natural Resources, and Design

at West Virginia University

in partial fulfillment of the requirements for the degree of

Doctorate of Philosophy in

Forest Resources Science

\begin{abstract}
Amy B. Welsh, Ph.D., Chair
James T. Anderson, Ph.D.

Richard E. Rogers, M.S.

Christopher W. Ryan, Ph.D.

Daniel G. Panaccione, Ph.D.
\end{abstract}

School of Natural Resources

Morgantown, West Virginia

2018

Keywords: Bobcat, Lynx rufus, hair snares, hair snare cubby, occupancy modeling, population genetics, Royle-Nichols model, Feline Panleukopenia Virus, Canine Parvovirus

Copyright 2018 Thomas F. Rounsville Jr. 


\section{ABSTRACT \\ The Bobcats of West Virginia: How Many and Where Are They?}

Thomas F. Rounsville Jr.

Bobcats (Lynx rufus) are mesocarnivore felids that are now distributed nearly throughout the entire United States. However, unsustainable harvests in the 1800s and early 1900s caused massive population declines from which bobcats have only recently recovered. Populations have recovered enough that they are harvested for their fur in many States. Since bobcats were listed under Appendix II of the Convention on International Trade in Endangered Species of Flora and Fauna (CITES) in 1977, states that have bobcat harvests are required to have management plans in place to ensure that harvest rates are sustainable. In West Virginia the current model for bobcat management uses population and density estimates from locations surveyed in neighboring Virginia. In order to ensure that harvest rates remain sustainable, new population estimates are required, as well as information on the statewide distribution of bobcats. To collect samples from wild bobcats a new non-invasive hair snare was developed: the bobcat hair snare cubby. Over the course of two field seasons in March - August of 2015 and March - July of 2016, a total of 1,500 sites $10 \mathrm{~km}^{2}$ in size were sampled for bobcats. A total of 378 bobcat detections were recorded, for an overall rate of 0.9 bobcat detections per 100 trap nights. Occupancy models were constructed using the data collected only in 2015 and the combined 2015 - 2016 dataset. Average predicted probability of detection $(p)$ was $0.133 \pm 0.027$ and 0.098 \pm 0.014 , for the 2015 and the 2015-16 datasets, respectively. The average predicted occupancy probability $(\psi)$ was $0.858 \pm 0.057$ and $0.834 \pm 0.055$, for the 2015 and 2015-16 datasets, respectively. Bobcats were nearly ubiquitously distributed throughout West Virginia, and of the tested hypotheses, human influences on the landscape had the greatest impact on both the $p$ and $\psi$ of bobcats. To estimate the size of West Virginia's bobcat population the combined 2015 2016 presence-absence dataset with the redone sites removed, and the best occupancy and detection models from 2015 - 2016 was used to construct a Royle-Nichols model. The size of the population was estimated at was 10,926.51 $\pm 2,960.43$, with a $95 \%$ confidence interval of 6,018.2 - 19,838.26. Of the total 378 bobcat detections, 230 successfully produced microsatellite genotypes and 212 bobcat individuals were found to be detected once while 9 individuals were detected twice. From the 2014 - 2015 bobcat hunting and trapping season a total of 304 bobcat carcasses were collected from successful hunters and trappers. During necropsy, whole spleen samples were removed from each animal and a subsample was taken for genetic analyses. A nine locus microsatellite genotype profile was successfully constructed for 280 samples. Using three separate methods, no population genetic structure with a geographical basis was detected. The effective population size was calculated to be roughly 1,448.7 with a jackknifed 95\% confidence interval of $426.8-\infty$. Both the Sign Test and the Wilcoxon one-tailed test for heterozygote deficiency supported a recent, statistically significant population bottleneck in West Virginia's bobcats. Additional bobcat carcasses were collected from the 2015 - 2016 hunting and trapping seasons, from which spleen samples were also removed for a total of 528. DNA testing was completed on the spleens to test for the presence of Feline Panleukopenia Virus (FPV) and Canine parvovirus (CPV). A total of $17.6 \%$ of spleens were found to be infected and were DNA sequenced to determine the viral type. CPV-2 / CPV-2a was detected in $85.4 \%$ of all positive samples, $11.5 \%$ were infected with CPV-2b, and 3.1\% were infected with FPV. 


\section{DEDICATION}

First, I would like to thank Dr. Amy Welsh for her mentorship, guidance, and support. Your dedication to my professional development has been inspiring. Thanks for always being there to provide helpful advice after listening to all of the struggles I experienced in each stage of this project. I thank Dr. Jim Anderson for providing his expertise to so many facets of this project. I am particularly grateful for your editing skills and for always being able to contribute even when timelines were tight. Thanks for pushing me to make this project as good as it could be. I also thank Rich Rogers of the West Virginia Division of Natural Resources (WVDNR) for sharing his seemingly endless wisdom on furbearer management. Thanks for always being just a phone call away whenever I had a question with current management methods, or I just wanted to share a particularly neat result. Also, I thank you for your encouragement and for always pushing me to be more confident when talking about my research. I am definitely going to miss working with you. To Dr. Chris Ryan, also of the WVDNR, I thank you for your help and support, especially when gathering materials to build the first batch of cubbies. I am also appreciative of your feedback and comments through each step of the project that made it into what it is today. I would also like to thank Dr. Dan Panaccione for teaching me the many considerations to take into account when designing primers and for enhancing my skills in molecular biology.

Second, I would like to thank the other graduate students at WVU for providing a supportive environment, especially when times were tough. Stephanie Landry - it was a pleasure working with you on the bobcat project and I also enjoyed our friendship. Thanks for always taking the time to help me with building cubbies, doing field work, or helping out in the genetics lab. Darren Wood - thanks for your help and support through the daily ups and downs of being a 
graduate student. I always appreciated our conversations about life, learning, and random stuff. I will never forget the fun pranks, or our travels to conferences far and wide. I would also like to thank the "Graduate Student Sport Group" that always took some time out of our busy schedules to play volleyball, racquetball, Frisbee, or softball. This exercise was always a great way to take a step back and think about research problems while having fun together. I will definitely miss the comraderie.

I would like to thank my family for the support over the many long years it has taken me to reach this achievement. Mom and dad, I know how much you love reminding me of all the times in my childhood when I said that I hated school and wanted to quit. And, here I am, all of these years later, just finishing school. Thanks for being there for me and for pushing me to keep at it, even when things got tough. There is no way I could have made it this far without your love and encouragement. To Rachel - thanks for being a great sister and for being there to talk to when things were tough. You have also been an inspiration for me to try my best at anything I do.

Last, but by no means least, I would like to thank my wife Mary and my best friend Josh. I know that this whole experience has been pretty rough on our relationships since I have not been around much these past couple of years. Josh - thank you for being a great friend and for being there to listen to all of my rants about academic life. Thanks for taking the time to go hiking and biking and just to do anything to get my mind away from issues with my project to help me feel refreshed. To Mary, thanks for being the absolute best support system that I could ask for. Thanks for being patient and understanding all of the times when I had to leave and go to the lab and for all of the long nights I spent toiling on this project away from you. Your love and 
encouragement was often the only thing that kept me going strong through all the toils of the Ph.D. Thanks for always being there for me. 


\section{ACKNOWLEDGEMENTS}

Financial support for this dissertation was provided by the West Virginia Division of Natural Resources through a grant with the U.S. Fish and Wildlife Service Wildlife and Sport Fish Restoration Program (W-48-R) and matching state funds.

This project would not have been possible without the many tireless hours contributed by the personnel of the West Virginia Division of Natural Resources. Thank you for your efforts in contacting landowners and running cubbies every week. I would also like to thank the many landowners that participated in this project and allowed us to sample for bobcats on their many land holdings. For this I am truly grateful.

Finally, I would like to thank the staff of the West Virginia Wildlife Center for assisting me in testing out the prototype cubby designs with the facility's bobcats. 


\section{Table of Contents}

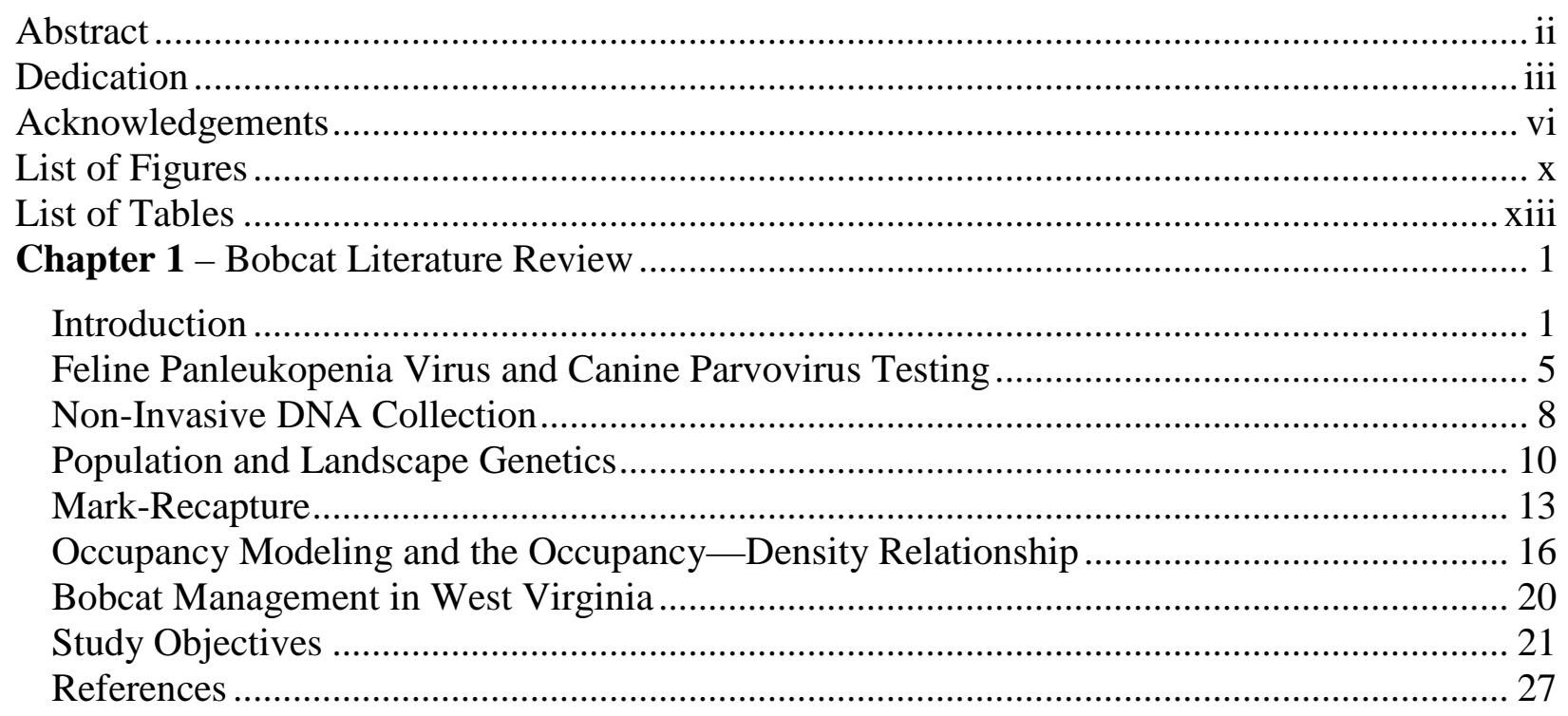

Chapter 2 - The Bobcat Hair Snare Cubby: A Non-Invasive Tool for Bobcat Population Monitoring ………………………………………………................................................. 35

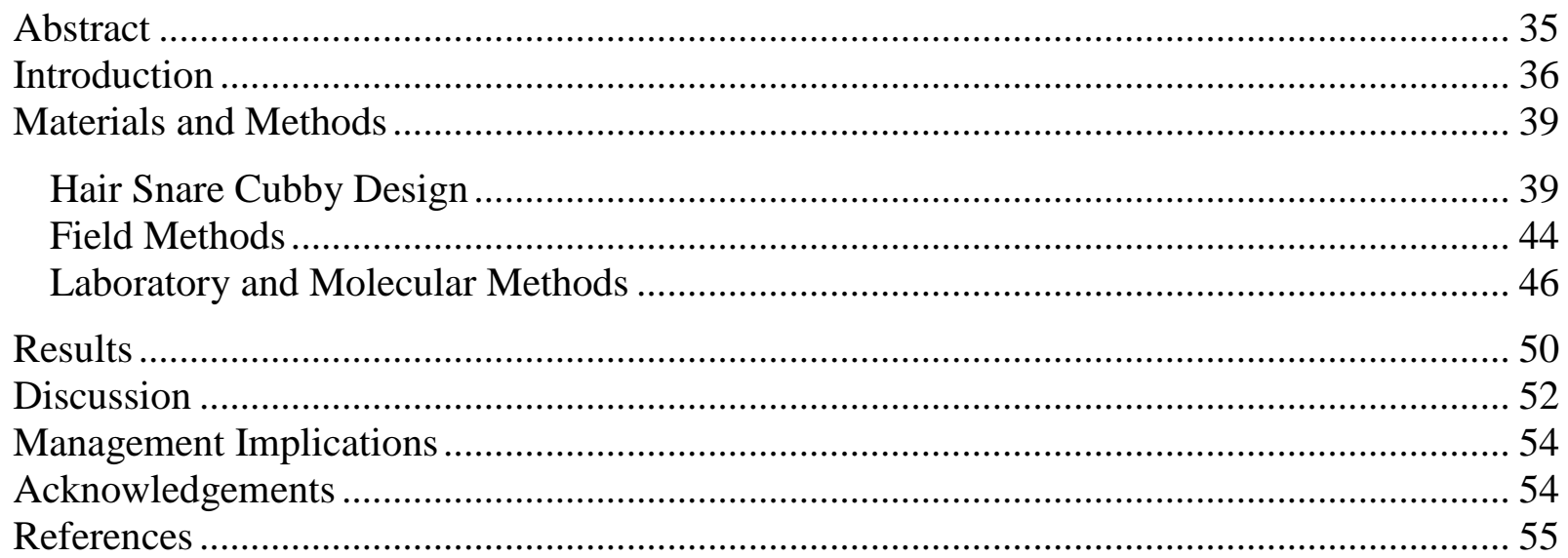

Chapter 3 - Modeling Bobcat Occupancy in West Virginia at the Landscape Scale ................... 57

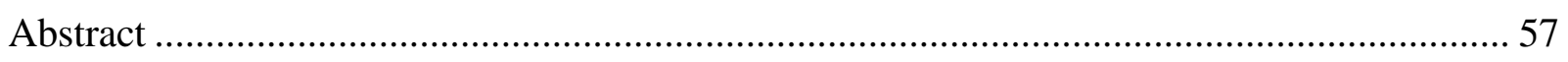

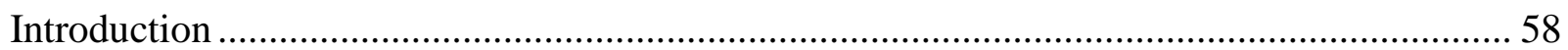

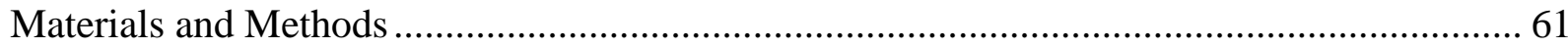

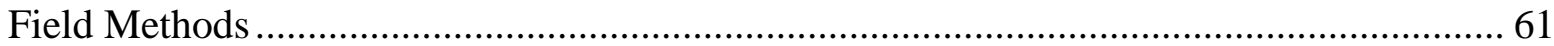

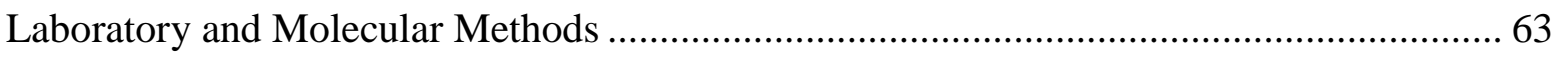

Occupancy Modeling........................................................................................................ 64

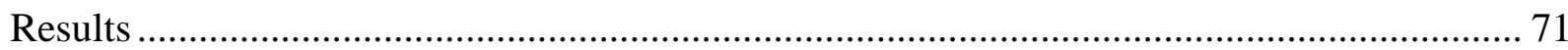

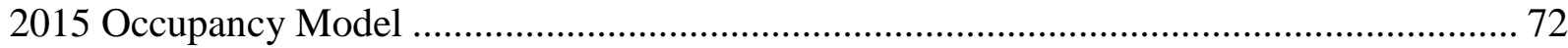

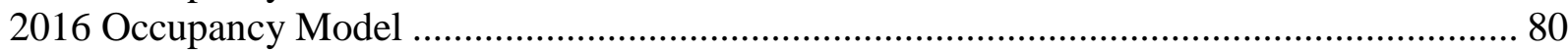

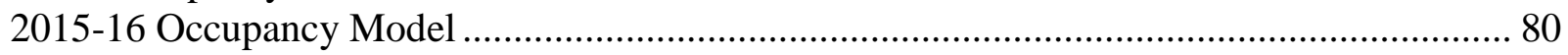




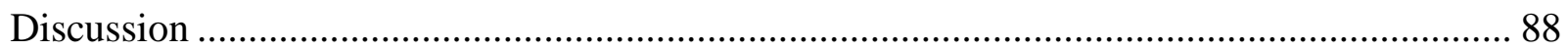

Predicted Estimates of Detection and Occupancy at the Landscape Scale ........................... 88

Best Covariates for Explaining Differences in Probability of Detection ................................ 89

Best Covariates for Explaining Variances in Bobcat Occupancy Probability......................... 91

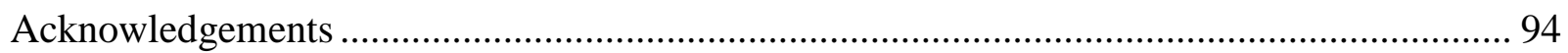

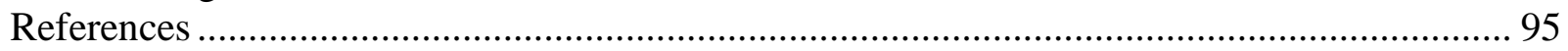

Chapter 4 - Estimating the Size of West Virginia’s Bobcat Population ..................................... 98

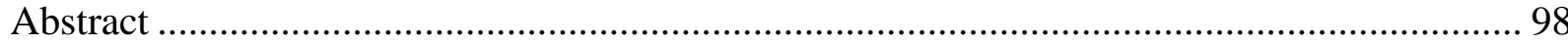

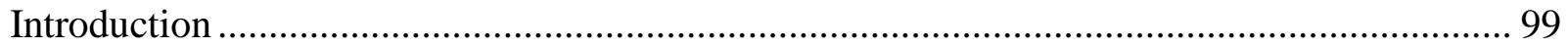

Spatially Explicit Capture-Recapture ........................................................................... 99

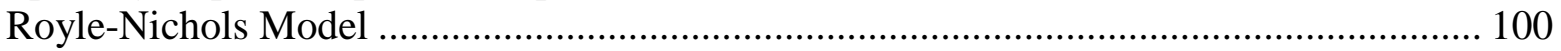

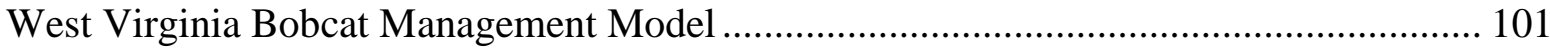

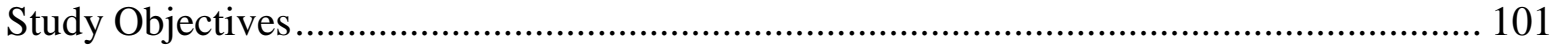

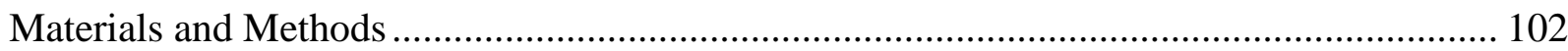

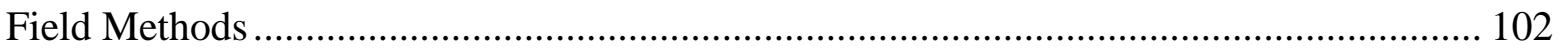

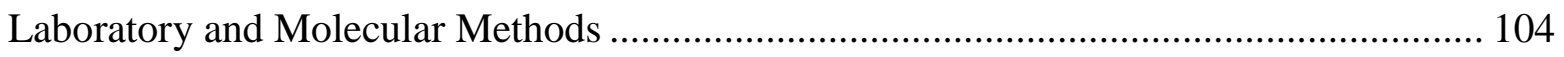

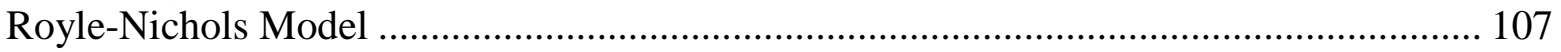

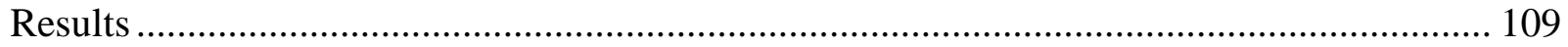

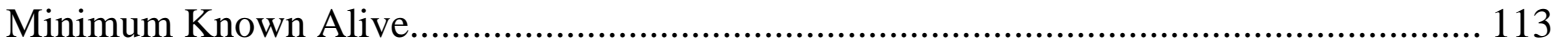

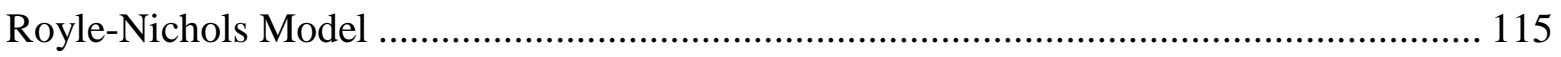

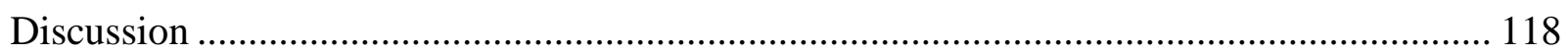

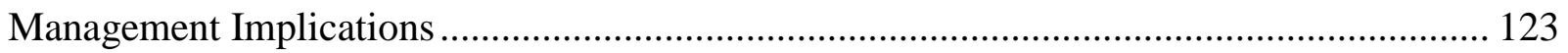

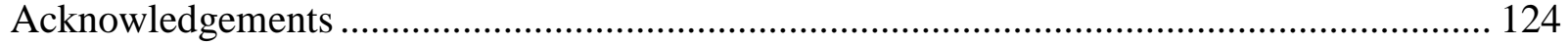

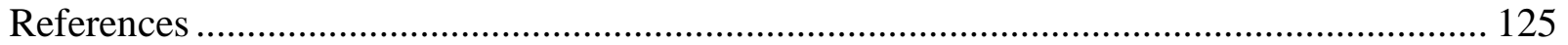

Chapter 5 - Population Genetics of West Virginia’s Bobcats ................................................... 128

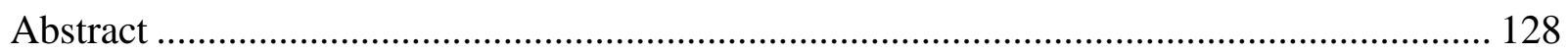

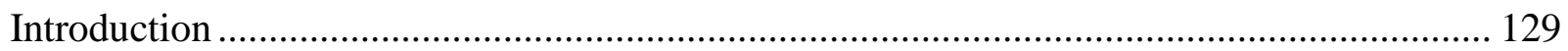

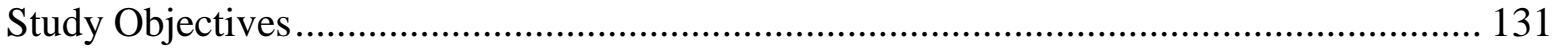

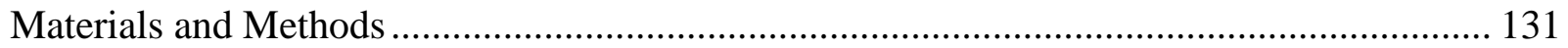

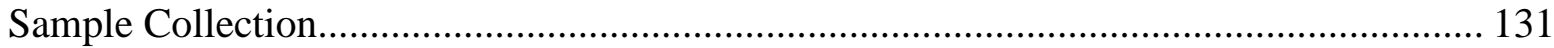

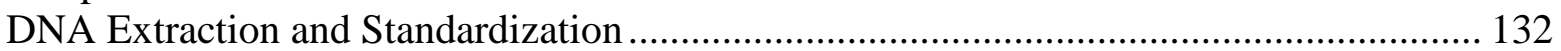

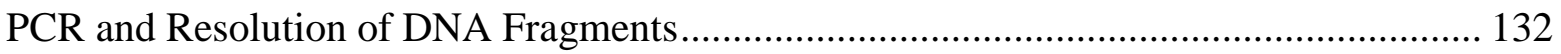

Genetically Distinct Population Identification .................................................................. 133

Basic Population Genetic Parameters............................................................................. 134

Effective Population Size Analysis .................................................................................. 135

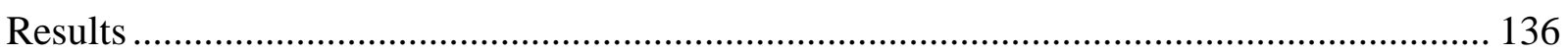

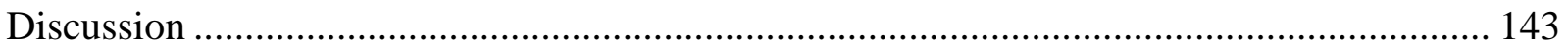

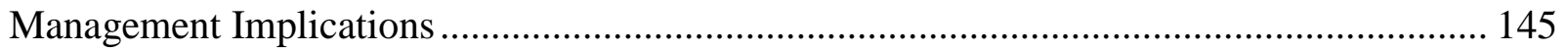

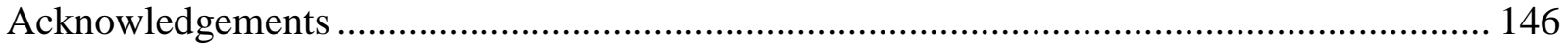

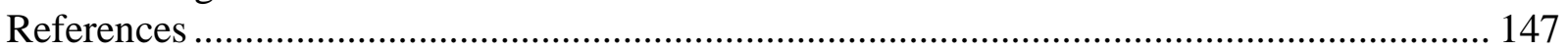


Chapter 6 - Prevalence and Molecular Characterization of Feline Panleukopenia Virus (FPV) and Canine Parvovirus (CPV) in Bobcats of West Virginia....................................................... 151

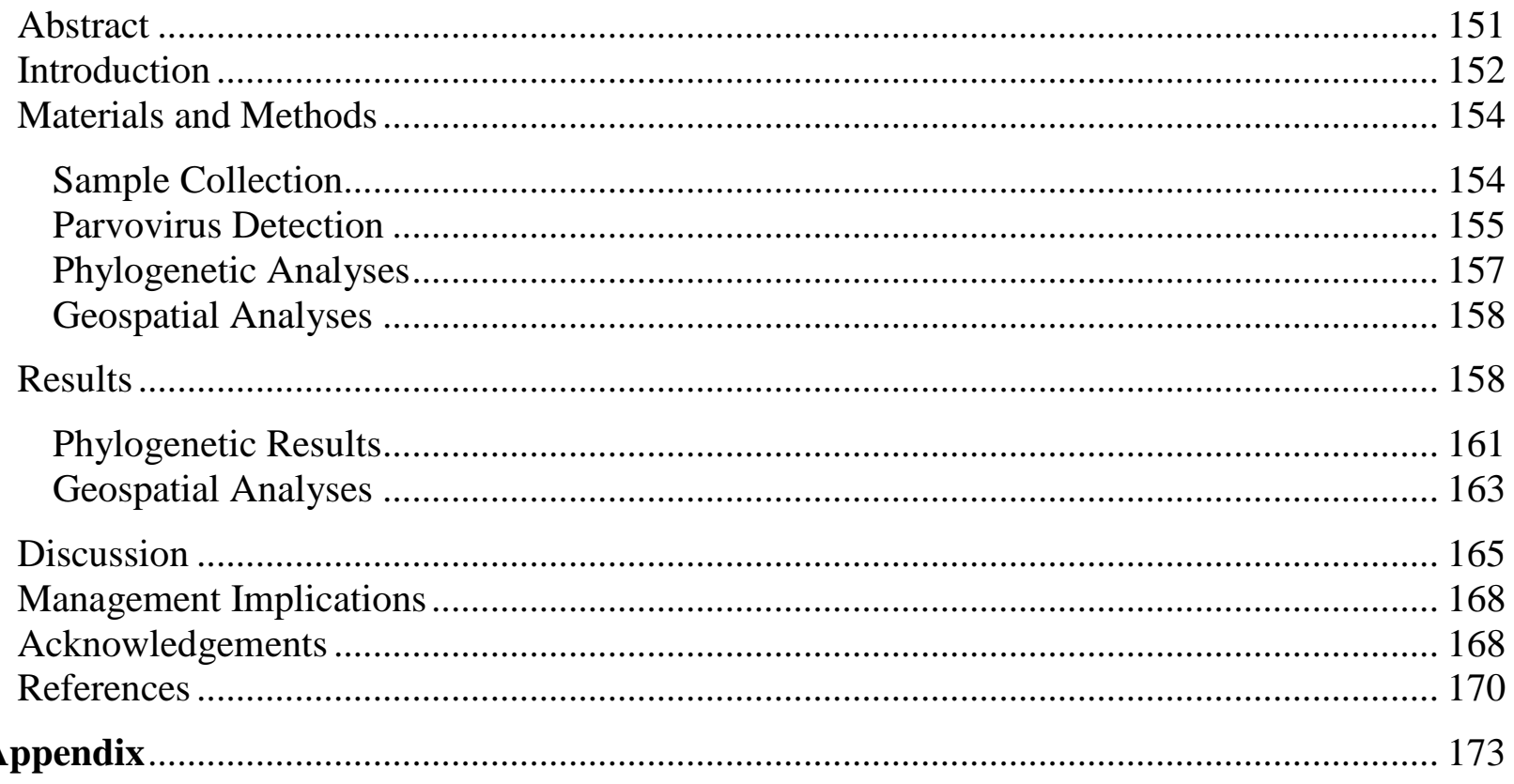




\section{List of Figures}

Figure 1 - Map depicting the current hunting and trapping yearly bag limits in West Virginia and all bordering states.

Figure 2 (A \& B) - (A) Bobcat hair snare cubby deployed in West Virginia. (B) Bobcat being sampled as it walks through the hair snare cubby.....

Figure 3 - Bobcat hair snare cubby construction schematic. The black dots on the collapsed view diagram indicate where perforations were made to affix the \#8-32 bolts to which the gun brushes were affixed. The bolt placement was staggered such that each lateral side of the cubby had one entrance where the bolt placement was low and one that was high. All bolts perforations were made at a distance of $2.6 \mathrm{~cm}$ from the outside edge of the sampling device. Solid black lines indicate where creases were made by folding the corrugated plastic against a solid object with a right angle, such as a laboratory benchtop. To create the lip needed for affixing the ground spikes, a second set of creased folds at the edge were required, on the opposite side of the corrugated plastic sheet as those used for the central folds. When erecting the cubby, spikes were driven through the four outside corners of the device at locations roughly $2.6 \mathrm{~cm}$ from any outside edges.

Figure 4 - Map of the sampled grid locations in West Virginia during the 2015 and 2016 field sampling seasons. Locations in blue were sampled only in 2015, orange sites were only sampled in 2016, and pink sites were sampled both in 2015 and 2016. A single hair snare cubby was placed in each of the cells of the 5x5 grid during sampling. Sampling took place at each location over the course of a four-week session. For both 2015 and 2016 a total of five sampling sessions were undertaken (Table 1). A total of six $5 \times 5$ grids were sampled simultaneously during the same session, with a total of 30 grids (7,500km2) being sampled in 2015 another 30 in 2016. .. 43

Figure 5 - A $10 \mathrm{~km}^{2}$ raster of West Virginia with pixels assigned the values of bobcat occupancy probability $(\psi)$ as predicted from the model-averaged 2015 occupancy model for covariates collected from centroid points of each polygon...................................................................... 78

Figure 6 - Inverse Distance Weighted (IDW) interpolated raster created using the values of bobcat occupancy probability ( $\psi$ ) predicted for each raster cell by the 2015 occupancy model. 79

Figure 7 - A $10 \mathrm{~km}^{2}$ raster of West Virginia with pixels assigned the values of the probability of bobcat occupancy $(\psi)$ as predicted from the 2015-16 occupancy model for the covariates collected from centroid points of each polygon. 86

Figure 8 - Inverse Distance Weighted (IDW) interpolated raster created using the values of predicted bobcat occupancy probability $(\psi)$ for each raster cell by the 2015-16 occupancy model. 
Figure 9 - Map of the locations surveyed for bobcats in West Virginia between March - August of 2015. Each 5x5 set of cells was sampled for a total of four weeks, with one bobcat hair snare cubby being placed in each cell. Cells marked with blue on the map are locations that were sampled but a bobcat was not detected at those locations. Cells marked in red indicate that at least one bobcat detection was recorded at that site. Background cells in tan were not sampled. The black lines on the map separate West Virginia into its separate ecological regions (appendix

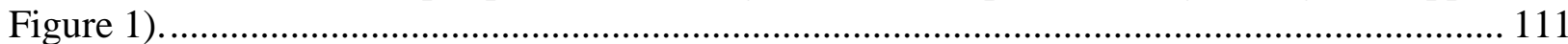

Figure 10 - Map of the locations surveyed for bobcats in West Virginia between March - July of 2016. Each $5 \times 5$ set of cells was sampled for a total of four weeks, with one bobcat hair snare cubby being placed in each cell. Cells marked with blue on the map are locations that were sampled but a bobcat was not detected at those locations. Cells marked in red indicate that at least one bobcat detection was recorded at that site. Background cells in tan were not sampled. The black lines on the map separate West Virginia into its separate ecological regions (appendix

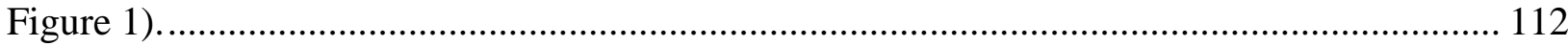

Figure 11 - Graph of $\Delta K$ and log likelihood $(\mathrm{LnP}(K))$ for each value of $K$ (hypothesized number of distinct genetic clusters) for 280 West Virginia bobcat samples genotyped at nine microsatellite loci. This graph was produced as the output of STRUCTURE Harvester (Earl and vonHoldt 2012) after analyzing the ten iterations completed for each value of $\mathrm{k}$ from $1-10$. However, values of $K>5$ were not graphed by STRUCTURE Harvester due to their significantly lower $\operatorname{LnP}(K)$ values. 137

Figure 12 (A \& B) - Output of the program STRUCTURE (Pritchard et al. 2000, Falush et al. 2003, Falush et al. 2007) when $K=3$ (a) and $K=2$ (b) for the 280 bobcats of West Virginia analyzed at nine microsatellite loci. Each bar represents one individual. The height of each color on the bar is the probability of assignment for that individual to that particular genetic cluster.138

Figure 13 - Principal Components Analysis (PCA) of Nei's pairwise genetic distance as calculated as a combined score across nine microsatellite loci from 280 bobcat samples from West Virginia. This PCA analysis was completed in GenAlEx v.6.503 (Peakall and Smouse 2012). Axis 1 contained 10.53\% of all variation and axis 2 contained 9.11\% of all variation, for a total of $19.64 \%$ of variation explained by the combination of these two axes. 140

Figure 14 - The Deviance Information Criterion (DIC) for each value of the maximum number of populations (Kmax) as outputted by TESS v2.3 (Chen et al. 2007, Durand et al. 2009) for 280 West Virginia bobcats genotyped at nine microsatellite loci.... 141

Figure 15 - Graphical output of population assignment probabilities for a second iteration of TESS v2.3 (Chen et al. 2007, Durand et al. 2009) for 280 West Virginia bobcats genotyped at nine microsatellite loci, when Kmax $=5$. 
Figure 16 - Map of West Virginia detailing the prevalence of Carnivore protoparvovirus 1 in each county from which bobcat carcasses were collected during 2014 - 2016. The size of the pie chart in each county is indicative of the number of samples collected from each county and the color of the pie slice indicates the prevalence rate of each viral type detected. The detailed information used to create this map can be found on Table 1 of the Appendix.

Figure 17 - Median-joining haplotype network as constructed using the program NETWORK 5.0.0.3 for Carnivore protoparvovirus 1 sequences amplified from bobcat spleens collected in West Virginia between 2014 - 2016. The size of each node is dependent on the number of samples that were found for each haplotype. Hashmarks on each linkage indicate the number of polymorphisms between the related haplotypes. Color of each pie slice is indicative of the ecological region (appendix Figure 1) from which each sample was collected. Outline color of the nodes indicates the viral typing of that particular haplotype. Nodes outlined in pink were determined to be FPV, those outlined in green were CPV-2b, and those outlined in black were CPV-2 / CPV-2a. 162

Figure 18 - Inverse Distance Weighted (IDW) interpolated map of Z-scores from the Getis-Ord Gi Hotspot Analysis of Carnivore protoparvovirus 1 prevalence rates for each county of West Virginia. Viral DNA sequences were amplified from bobcat spleens collected from 2014 - 2016. Locations in red indicate areas where prevalence of Carnivore protoparvovirus 1 is much greater than would be expected in an even distribution and locations in blue indicate areas where prevalence of Carnivore protoparvovirus 1 is less than what would be expected in an even distribution. Locations in yellow are those that have no statistically significant relationships.. 164

Appendix Figure 1 - Map of the ecological regions of West Virginia, as described by Uhlig and Wilson (1952). 173

Appendix Figure 2 - Graph of the correlation between the human population density of each West Virginia county from which the prevalence of Carnivore protoparvovirus 1 was calculated. Prevalence rates were determined as the number of bobcat spleens collected from 2014 - 2016 from which Carnivore protoparvovirus 1 DNA was successfully sequenced as compared to all samples tested. 


\section{List of Tables}

Table 1 - Sampling dates for each session for both the 2015 and the 2016 sampling seasons. .. 44

Table 2 - Comparison of the detection rate performance of the bobcat hair snare cubby with other hair snares specifically designed to sample bobcats.

Table 3 - Covariates used to model probability of detection $(p)$ and occupancy probability $(\psi)$ in bobcats of West Virginia. The predictor type is the parameter estimates for which that covariate was used. The expected result is the direction of the correlative relationship between each covariate and bobcat $p$, $\psi$, or both. Hypotheses associated with conclusions reached from other studies are marked with a superscript.

Table 4 - Selected models for detection probability $(p)$ using bobcat detections recorded in West Virginia during the 2015 season. -2 Ln L is 2 times the negative log likelihood of that particular model. $\triangle \mathrm{AIC}=$ Akaike's Information Criterion. $\omega \mathrm{r}$ is the model weight ratio, recalculated for these selected models. $\mathrm{K}^{*}$ is the number of parameters included in the model. This number is greater than the number of parameters listed under model because SESSION has to be coded as four separate variables (SESSION 1 was reference and absorbed into the intercept and SESSIONS 2, 3, 4, and 5 were included) in RPRESENCE. (For covariate definitions, see Table 3).

Table $5-\beta$ values (with standard errors) for the BEST model for detection probability ( $p$ ) of the 2015 dataset and the included covariates (Table 3). This was then held constant when modeling occupancy $(\psi)$ for the 2015 dataset. Values in bold indicate that the parameter estimate, when combined with the standard error, did not cross zero, and is a significant predictor.

Table 6 - Selected models for the probability of occupancy $(\psi)$ and the 2015 dataset of bobcat detections in West Virginia. -2 Ln L is 2 times the negative log likelihood of that particular model. $\triangle \mathrm{AIC}=$ Akaike's Information Criterion. $\omega \mathrm{r}$ is the model weight ratio, recalculated for these selected models. $\mathrm{K}^{*}$ is the number of parameters (Table 3 ) included in the model. This number is greater than the number of parameters listed under model because SESSION has to be coded as four separate variables (SESSION 1 was reference and absorbed into the intercept and SESSIONS 2, 3, 4, and 5 were included) in RPRESENCE.

Table $7-\beta$ values (with standard errors) for the selected models for occupancy probability ( $\psi$ ) of the 2015 West Virginia bobcat detection dataset and the included covariates (Table 3). Values in bold indicate that the parameter estimate, when combined with the standard error, did not cross zero, and is a significant predictor. $\mathrm{F}$ is the number of selected models in which that covariate is a significant predictor. 
Table 8 - Selected models for detection probability ( $p$ ) using the 2015-16 dataset of bobcat detections in West Virginia. -2 Ln L is 2 times the negative log likelihood of that particular model. $\triangle \mathrm{AIC}=$ Akaike's Information Criterion. $\omega \mathrm{r}$ is the model weight ratio, recalculated for these selected models. $\mathrm{K}^{*}$ is the number of parameters (Table 3 ) included in the model. This number is greater than the number of parameters listed under model because SESSION has to be coded as four separate variables (SESSION 1 was reference and absorbed into the intercept and SESSIONS 2, 3, 4, and 5 were included) in RPRESENCE.....

Table $9-\beta$ values (with standard errors) for the BEST model for detection probability ( $p$ ) of the 2015-16 dataset and included covariates (Table 3). This was then held constant when modeling the probability of occupancy $(\psi)$ for the 2015-16 West Virginia bobcat presence-absence dataset. Values in bold indicate that the parameter estimate, when combined with the standard error, did not cross zero, and is a significant predictor. 83

Table 10 - Selected models for occupancy probability ( $\psi$ ) using the 2015-16 West Virginia bobcat presence-absence dataset. $-2 \mathrm{Ln} \mathrm{L}$ is 2 times the negative log likelihood of that particular model. $\triangle \mathrm{AIC}=$ Akaike's Information Criterion. $\omega \mathrm{r}$ is the model weight ratio, recalculated for these selected models. $\mathrm{K}^{*}$ is the number of parameters (Table 3 ) included in the model. This number is greater than the number of parameters listed under model because SESSION has to be coded as four separate variables (SESSION 1 was reference and absorbed into the intercept and SESSIONS 2, 3, 4, and 5 were included) in RPRESENCE.................................................. 85

Table $11-\beta$ values (with standard errors) for the BEST model for probability of detection ( $p$ ) of the 2015-16 dataset of West Virginia bobcat presence-absence and the included covariates (Table 3 ). This was then held constant when modeling $\psi$ for the 2015-16 dataset. Values in bold indicate that the parameter estimate, when combined with the standard error, did not cross zero, and is a significant predictor. 85

Table 12 - Covariates used to model probability of detection $(p)$ and occupancy probability $(\psi)$ in the bobcats of West Virginia. The predictor type is the parameter estimates for which that covariate was used. The expected result is the direction of the correlative relationship between each coavariate and bobcat $\mathrm{p}, \psi$, or both. Hypotheses associated with conclusions reached from other studies are marked with a superscript.

Table 13 - The number of sites sampled, number of bobcat detections, number of unique individuals sampled, minimum bobcat density, mean ecoregion (appendix Figure 1) detection probability and minimum bobcat density averaged across each ecoregion when taking detection probability into account for bobcat hair samples collected in West Virginia between 2015 2016. 
Table 14 - Selected models for abundance $(\lambda)$ and detection probability $(p)$ using the 2015-16 and just 2015 West Virginia bobcat presence-absence dataset. -2 Ln L is 2 times the negative log likelihood of that particular model. $\triangle \mathrm{AIC}=$ Akaike's Information Criterion. $\omega r$ is the model weight ratio, recalculated for these selected models. $\mathrm{K}^{*}$ is the number of parameters (Table 12) included in the model. This number is greater than the number of parameters listed under model because SESSION has to be coded as four separate variables (SESSION 1 was reference and absorbed into the intercept and SESSIONS 2, 3, 4, and 5 were included) in RPRESENCE. .... 117

Table 15 - $\beta$ values (with standard errors) for the BEST model from the 2015 - 2016 combined dataset for probability of detection $(p)$ and occupancy $(\psi)$ of the West Virginia bobcat presenceabsence and the included covariates (Table 12). This model was then used to model abundance in a Royle-Nichols framework using the program PRESENCE. Values in bold indicate that the parameter estimate, when combined with the standard error, did not cross zero, and is a significant predictor.

Table 16 - Allelic richness (A), observed heterozygosity $\left(\mathrm{H}_{\mathrm{O}}\right)$, expected heterozygosity $\left(\mathrm{H}_{\mathrm{E}}\right)$, and p-value for test of Hardy-Weinberg Equilibrium deviation significance $\left(\mathrm{HWE}_{\mathrm{p}}\right)$ for each examined microsatellite locus of a 280 bobcat dataset collected in West Virginia between 2014 2015. $\mathrm{HWE}_{\mathrm{p}}$ values with an asterisk indicate those that were found to be significantly out of Hardy-Weinberg Equilibrium by CERVUS v.3.0 after using a Bonferroni correction $(\alpha=$ 0.0056).

Appendix Table 1 - For each location sampled during the 2015 - 2016 bobcat hair snaring seasons in West Virginia, the following data are recorded: the grid number, year the grid was sampled, the county sampled, the ecoregion in which this county is located (appendix Figure 1), the number of bobcat detections recorded by qPCR analysis, the calculated 4-week detection probability as estimated by the previously constructed occupancy model (Chapter 3 ) for that particular grid, the minimum known alive (MKA), the minimum density of bobcats calculated for $100 \mathrm{~km} 2$, the minimum number of bobcats calculated for $100 \mathrm{~km} 2$ when taking the detection probability for that grid into account (100km2 DET), and whether or not a location sampled in 2016 was previously sampled in 2016 (Redo).

Appendix Table 2 - A detailed account of the number of bobcat spleen samples tested from each participating West Virginia county and the findings of the tests. Spleen samples were collected from bobcats harvested between 2014 - 2016 and samples confirmed as testing positive produced a DNA sequence consistent with that of Carnivore protoparvovirus 1. 176

Appendix Table 3 - This table contains the raw data used to create the proportion charts on the Carnivore protoparvovirus 1 haplotype network constructed in this study (Chapter 6, Figure 2) for DNA sequences isolated from bobcat spleens collected in West Virginia between 2014 2016. A map of the ecological regions of West Virginia is included in this Appendix as figure 1. 


\section{Chapter 1 - Bobcat Literature Review}

\section{Introduction}

Lynx rufus, commonly known as the bobcat, is a medium sized member of the cat family (Felidae). Traditionally, the range of the bobcat encompassed the majority of the continent of North America from Southern Canada into Mexico (Ray 2000). Currently, the bobcat occupies much of this historical range due largely to its ability to adapt to areas of human disturbance.

While the historic and current range of bobcats may be similar, the prevalence of bobcats in these areas has varied significantly over the past century. In the 1800 s bobcats were considered a nuisance species because of their predatory habits. As a result, many states instituted bounty programs to reduce the overall population densities (Litvaitis et al. 2006). For example, a bobcat bounty program with no closed season in New Hampshire was instituted in 1809 and remained in effect nearly continuously until 1974 when bobcats were awarded game species status. The number of harvested bobcats dropped substantially in the 1960s and 1970s, leading to eventual protected status of bobcats in that state. West Virginia, the focal location of this study, had a similar bounty program in place until 1961 (personal communication: Rich Rogers, West Virginia Division of Natural Resources), with roughly 10 pelts per year sold by trappers in the state in the late 1960s. At that time, West Virginia's bobcat population was then considered to be consistently low based on lack of trapping success, and as such, bobcats were granted game species status with a four-month trapping season in 1974. Since being afforded these protections, the bobcat population within West Virginia has been increasing as evidenced by increasing nuisance complaints, harvests, and harvest rates (personal communication: Rich Rogers, WVDNR). 
Bobcat abundance in the United States has been on the rise since the 1980s (Roberts and Crimmins 2010), and since then many states have instituted more liberal harvest seasons and bag limits for bobcats. However, many states still lack credible bobcat density estimates. Roberts and Crimmins (2010) surveyed wildlife managers in the lower 48 states, as well as Canada and Mexico, asking managers to provide information about bobcats found within their jurisdictional areas. Of the 47 state wildlife management agencies that responded to the survey, 21 of these agencies had no available estimate for the number of bobcats residing within their state. Even though some states did not have an estimate of the number of bobcats within their jurisdiction, $96 \%$ of these management agencies estimated that the bobcat population in their state either had increased or stabilized since 1981, based on data collected using one or more of the following methods: public sightings; harvest analyses and population modeling; hunter surveys; and scent, scat, track, or road kill indices.

Regarding conservation status, the recent recovery of bobcat populations across North America has resulted in their classification as Least Concern by the International Union for Conservation of Nature and Natural Resources (IUCN) (Kelly et al. 2008). However, bobcats were included in the Convention on International Trade in Endangered Species of Wild Fauna and Flora (CITES) Appendix II in 1977, which requires careful monitoring of the hunting and trading of the pelts of these animals, since they are a look-alike species to the endangered Iberian lynx (Lynx pardinus) (CITES $15^{\text {th }}$ Meeting 2010). Recent proposals to remove the bobcat from CITES Appendix II by the United States government have been unsuccessful, even though bobcats are not deemed a species of conservation concern. While not afforded any protections at the federal level, bobcats are still a protected species in some states such as Ohio where the bobcat was recently removed from the state’s endangered species list (Ohio Department of 
Natural Resources 2014); however, as of 2018, a harvest season for eastern Ohio bobcats has been proposed by the Ohio Department of Natural Resources (Ohio Department of Natural Resources 2018).

While bobcats are one of the most widely distributed carnivores in North America (Roberts and Crimmins 2010), harvest management regimes deviate significantly from one location to another (Figure 1). For instance, West Virginia has a bobcat trapping and hunting season with a yearly bag limit of three individual bobcats (West Virginia Division of Natural Resources, 2014). West Virginia's neighboring states range from having closed bobcat seasons in Ohio (Ohio Department of Natural Resources, 2014) and Maryland (Maryland Department of Natural Resources, 2014) to a high of a 12 bobcat yearly bag limit in Virginia (Virginia Department of Game and Inland Fisheries, 2014). 


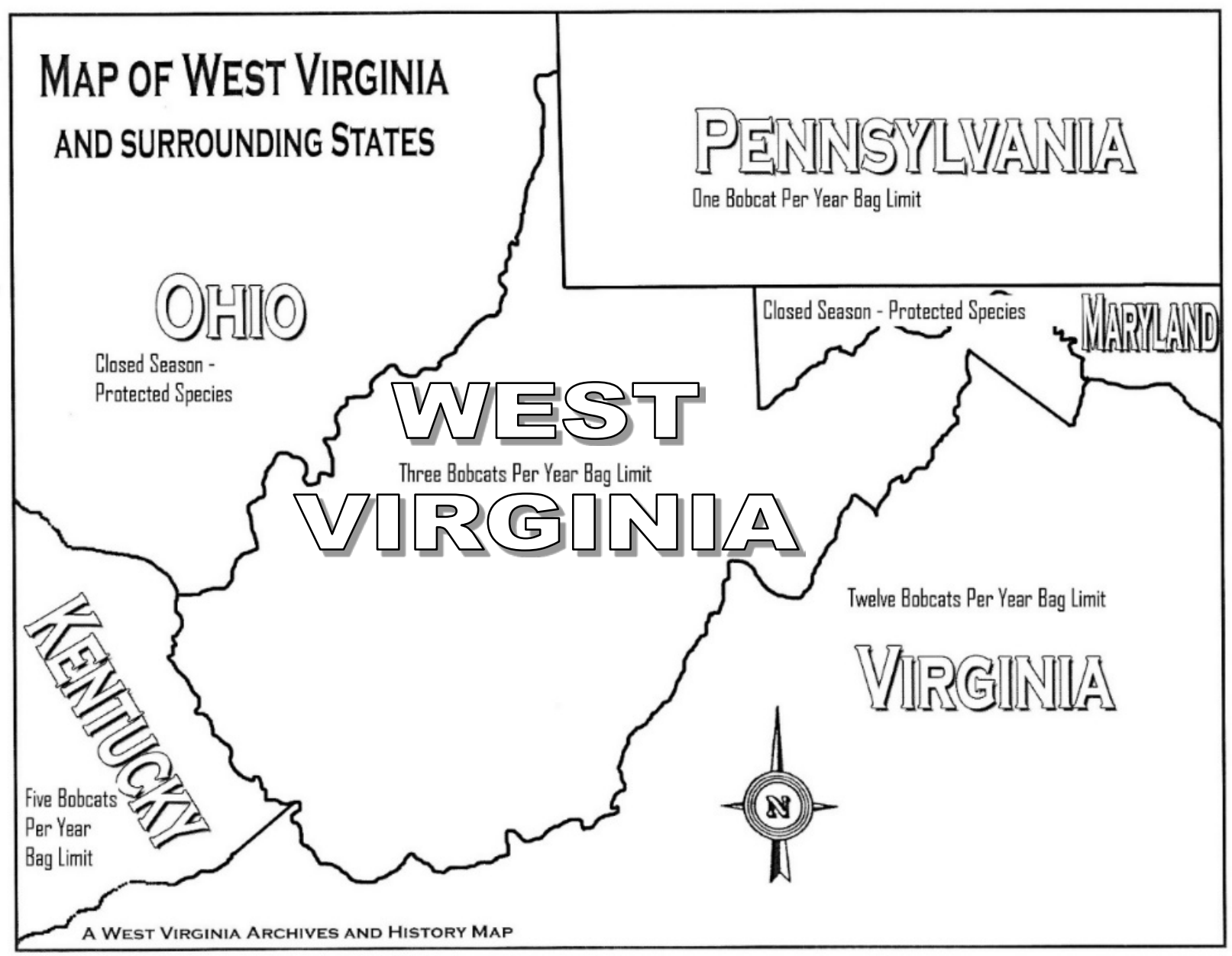

Figure 1 - Map depicting the current hunting and trapping yearly bag limits in West Virginia and all bordering states.

Bobcats live the majority of their lives in solitary home ranges that rarely overlap except for males overlapping with females in some areas (Bailey 1974). Home ranges are maintained with scent-marking and minimal interspecific conflict. When previously used home ranges become unoccupied due to the removal of the previous resident, another bobcat of the same gender will move into this area and establish a home range of roughly the same size (Lovallo and Anderson 1995, Benson et al. 2004). This was determined through experimental removal of bobcats in Mississippi and identification of other neighboring individuals expanding into this area (Benson et al., 2004). When juvenile bobcats disperse from their natal habitat, they may 
move into previously unused areas, if they are available (Kitchings and Story 1984). Dispersal occurs in both genders of juveniles, but males are more likely to disperse a greater distance than females, which exhibit some level of philopatry (Janečka et al. 2007, Croteau et al. 2010). Johnson et al. (2010) found that in south-central Indiana, only 42\% of dispersing juveniles were able to establish a home range. Additionally, individuals attempting to establish home ranges in fragmented habitats were often unsuccessful and were at risk of death by vehicle collisions.

When averaged across most available habitats in North America, male bobcats were found to have larger mean home ranges $\left(47.1 \pm 7.58 \mathrm{~km}^{2}\right)$ than females $\left(23.9 \pm 7.58 \mathrm{~km}^{2}\right)$, with the average bobcat home range irrespective of gender being $34.0 \pm 5.37 \mathrm{~km}^{2}$ (Elizalde-Arellano et al. 2012). However, at northern latitudes these numbers can vary significantly, even across seasons, as resource availability drives expansion or contraction of home ranges (Reed 2013). Of the radio-collared animals used in this study, females had a mean home range of $23.84 \mathrm{~km}^{2}$, while males had a mean home range size of $81.6 \mathrm{~km}^{2}$. The scent marking and home-range use patterns of bobcats indicate that they socially operate under a premise known as land-tenure (Benson et al. 2004). While extensive research has been conducted at the local scale to determine bobcat movement patterns and home ranges, little research focus has been paid to the large-scale movements of bobcats into areas from which they were previously extirpated.

\section{Feline Panleukopenia Virus and Canine Parvovirus Testing ${ }^{1}$}

The feline panleukopenia virus (FPV) is a member of the viral family Parvoviridae and was initially described in the 1920s (Siegl et al. 1985). Infection with FPV clinically manifests in felids as a severe hemorrhagic gastroenteritis that is often fatal—especially in young animals

\footnotetext{
${ }^{1}$ This section of the dissertation was co-authored by Stephanie M. Landry as a part of her M.S. degree at WVU
} 
(Schunck et al. 1995, Horiuchi et al. 1996, Kruse et al. 2010). Common symptoms of FPV include lethargy, leukopenia and lymphopenia (low white blood cell counts), fever, diarrhea, and vomiting. Once an infection becomes established, viral particles are shed at high titers in feces, urine, saliva, nasal and ocular discharge, and vomit for roughly three weeks post-infection (Csiza et al. 1971). Discharged viral particles remain infectious in the environment for up to a year (Wasieri et al. 2009, Foley et al. 2013). Viral transmission generally occurs via the fecal-oral route (Schunck et al. 1995) but can also occur through contact of mucous membranes with infected individuals or objects on which viruses have been shed (Wasieri et al. 2009).

While originally discovered in felids, mutations of this single-stranded DNA virus led to new FPV variants that infect a number of different host species (Allison et al. 2013). The most important of these variants is the virus known as canine parvovirus (CPV), which causes similar gastroenteritis symptoms and mortality in canids. CPV was originally discovered in the late 1970s (Decaro et al. 2010, Clegg et al. 2012, Allison et al. 2013) and at that time there was only one variant of the virus which was known as CPV-2. Since the discovery of CPV, the variant CPV-2 has disappeared from its worldwide distribution and it has been replaced by three new variants CPV-2a, CPV-2b, and CPV-2c (Decaro et al. 2010). The rapid evolution of CPV, as opposed to FPV, is thought to have been caused by the mutations in the capsid protein gene that differentiate the two viruses, and that this gene is under positive selection in CPV (Hoelzer et al. 2008).

The new variants of CPV are important to felids because they can cross the species barrier back into felids and cause disease, unlike the original CPV-2 (Battilani et al. 2007). Recent research has shown that many clinical cases of FPV are actually caused by a variant of CPV-2 in felids (Hoelzer et al. 2008, Decaro et al. 2010, Miranda et al. 2014). Ikeda et al. (2000) 
concluded that CPV may be spread more easily between felids than FPV, when they found that 80\% of viral isolates from domestic cats and leopard cats (Prionailurus bengalensis) were CPV2a/c, not FPV. Clegg et al. (2012) found that of the 230 felines sampled for FPV and CPV at an animal shelter, none were infected with FPV while roughly 33\% tested PCR-positive for CPV. CPV and FPV were traditionally considered to be different viruses; however, recent genetic systematics research has resulted in taxonomical changes and both viruses have been reclassified together as Carnivore protoparvovirus 1, along with the mink enteritis virus (MEV) and raccoon parvovirus (RaPV) (Cotmore et al. 2014).

While CPV and FPV appear to pose the greatest threat to domestic species, these viruses also pose an enormous threat to wild felids. Non-domestic felids can be infected by CPV and FPV and are susceptible to disease_-particularly young or already sick individuals. Wasieri et al. (2009) described a case study of an Eurasian lynx (Lynx lynx) and an European wildcat (Felis silvesteris) that died of the complications caused by an FPV infection spread from wild domestic cats living in the area. A serological survey of mountain lion adults in California found that 39\% had been exposed to FPV (Foley et al. 2013), which may be a threat to the survival of their offspring. An FPV outbreak in South Africa was responsible for the deaths of captive-bred nondomestic felids, and while not able to be directly proven, it was suspected that the source of the infection was feral domestic cats (Lane et al. 2016). The phylogenetic research of parvoviruses by Allison et al. (2012, 2013, 2014) concluded that FPV and CPV transmission between domestic and wild species appears to be commonplace and bi-directional, with the most likely route of transmission being predation events or scavenging. However, the extent of the threat that domestic animals present to wild felids is something that has not yet been quantified. 
Additional research needs to be conducted to determine how CPV and FPV is transferred between domestic and wild populations (Wasieri et al 2009, Foley et al. 2013, Lane et al. 2016). As human encroachment and development into wildlands continues, interactions between domestic and wild animals are becoming more common and the threat of domestic wildlife diseases negatively impacting wildlife populations becomes greater.

\section{Non-Invasive DNA Collection}

Over the past two decades, advances in genetic technology have resulted in a shift in the methodologies used to collect samples for wildlife studies. Non-invasive sampling techniques such as camera traps, scat transects, and hair snares are now the most commonly used to collect bobcat population information. Camera traps have been successfully used to document bobcat presence and estimate abundance or density when individual identification is possible from images (Heilbrun et al. 2003, 2006, Larrucea et al. 2007, Long et al. 2007, Symmank et al. 2008, Comer et al. 2011, Clare et al. 2015, Lesmeister et al. 2015, Lewis et al. 2015, Thornton and Pekins 2015, Lombardi et al. 2017). Camera trap costs have declined significantly since the first study conducted in 2003; however, using cameras to survey large areas still requires a sizable investment of resources. Scat transects, conducted using both human observers (Ruell et al. 2009, Morin et al. 2018) and detection dogs (Harrison 2006, Long et al. 2007), have also been successfully applied to the estimation of bobcat abundance, density, or occupancy. When directly comparing these two methods, scat detection dogs were found to be the most effective at finding bobcat samples, but they also had the greatest associated costs (Long et al. 2007, Harrison 2010), limiting the size of the overall study area. In contrast, hair snares have the lowest deployment 
costs, allowing for a much larger area to be surveyed with the same amount of expenditures. For large-scale studies, hair snares are often the only cost-effective option available.

The use of hair snares in wild felid research came into prominence after the development of the carpet scratch pad by McDaniel et al. (2000), which was successful in collecting hair samples from Canada lynx (Lynx canadensis). The device they developed was effectively used to study other species such as ocelot (Leopardus pardalis) (Weaver et al. 2005). Little success was reported when using the same carpet scratch pads to specifically target bobcats. In New Mexico, Harrison (2006) installed the carpet scratch pads as described in McDaniel et al. (2000) and achieved only one detection over 700 trap nights, even though bobcats were known to inhabit the study area. Long et al. (2007) deployed these same devices in known bobcat habitat in Vermont for 2,072 trap nights and failed to achieve any detections. More recently, Comer et al. (2011) collected hair from only one bobcat in 1,680 trap nights in eastern Texas, even though bobcats were detected by cameras at 15 carpet scratch pad sites. Comer et al. (2011) found that bobcats did not exhibit the rubbing response that was characteristic of Canada lynx, and thus were not sampled by the devices designed by McDaniel et al. (2000). Recent research using both cameras and the Canada lynx hair scratch pads found that hair snares had less than half the detection probability as compared to remote cameras, both located at the same sites (Crowley and Hodder 2017).

Due to the poor performance of the carpet scratch pads, most recent bobcat studies (Erb et al. 2012, Nogeire et al. 2013, Clare et al. 2015, Lesmeister et al. 2015, Lewis et al. 2015, Thornton and Pekins 2015, Wang et al. 2015, Lombardi et al. 2017) have used remote cameras, which is now the preferred sampling method. However, one recently published study has used a new approach to attempt to collect bobcat hair. In the upper peninsula of Michigan, Stricker et al. 
(2012) deployed a modified cable snare (Depue and Ben-David 2007) that was designed as a single-sample device placed in areas surrounding sites baited with deer carcasses. This device was constructed specifically as a passive device to collect hair samples without the required cheek rubbing behavior of the carpet scratch pad. Over the course of an 8-week study in a $278.5 \mathrm{~km}^{2}$ study area, a total of 230 hair samples were collected, of which 17 originated from bobcats, for an overall detection rate of 0.189 bobcats per 100 trap nights. This was the first study that documented the successful use of hair snares to sample wild bobcats and estimate population size by using a novel hair snaring method; however, their method is not feasible for heavily forested, mountainous terrain, like that found in West Virginia.

\section{Population and Landscape Genetics}

Population genetics is the study of allele frequencies within and between different populations of the same organism. The direct application of this field to wildlife management is that it can be used to determine genetically distinct subsets of a certain species or it can be used investigate how animals move across the landscape with much greater detail than can be done with traditional telemetry studies. Also of relevance to this project is a relatively new application of population genetics, landscape genetics, which is defined as the study of how heterogeneity of the landscape impacts allele frequencies in a population. Manel et al. (2003) published the first synthesis of techniques available to study this new field, with a specific emphasis on identifying how landscape features can enhance or prevent gene flow. While this paper represented the emergence of this new field, it was not until years later that the quantitative requirements of landscape genetics were made (Storfer et al. 2007). 
In the most recent synthesis on the topic, a textbook by Balkenhol et al. (2015), the authors establish three analytical requirements to meet when completing a landscape genetics study: 1) a concrete measurement of genetic variation amongst individuals, 2) a method to quantify landscape variation, and 3) a statistical method to quantitatively link genetic and landscape variation. The population and landscape genetics studies highlighted in this review will focus mainly on the detection of barriers to gene flow in bobcats in different locales across North America.

While not at the scale of resolution for regional management purposes, a significant study on the population genetics of bobcats across the United States was completed by Reding et al. (2012). This study analyzed portions of the mitochondrial genome (mtDNA) and 15 microsatellite loci for over 1,700 samples to determine the genetic differentiation in bobcats at the continental scale. Bobcats of the eastern and western United States were genetically divergent even though no formal boundary to gene flow was detected. At the regional scale including West Virginia, a Bayesian clustering analysis found a genetically differentiated population of bobcats in Pennsylvania, but West Virginia and the other surrounding states were clustered into a group known as "southeastern.” This evidence suggests that there is some potentially significant genetic differentiation between West Virginia and Pennsylvania, but not enough samples were collected from this transition zone to be conclusive. Reding et al. (2012) also speculated that, when looking at a continental scale, anthropogenic influences were most dramatic in the eastern United States and these eastern bobcats were not as genetically homogenous as western bobcats. It is this lack of genetic homogeneity in the eastern United States which may also indicate a patchy distribution of bobcats within and between the areas surrounding West Virginia. 
In another range-wide study of the population genetics of bobcats in North America, Croteau et al. (2012) used both microsatellite genotyping and mitochondrial haplotyping to investigate genetic differentiation. The authors found significant genetic structure between the bobcats in the eastern and western parts of North America using mtDNA. Genetic structure was also documented when comparing bobcats of the Midwest and eastern North America populations revealing the presence of barriers to gene flow at this large landscape scale. A microsatellite genotyping project conducted in Ohio found that the bobcats inhabiting eastern Ohio are genetically differentiated from neighboring populations in western Ohio, Pennsylvania, West Virginia, and Kentucky (Anderson et al. 2015). Rather than being the remnants of a reemerging population, the authors found evidence of a slight founder effect and hypothesized that the bobcat population now found in eastern Ohio is the result of movements of individuals in the area from source populations in other states.

In addition to bobcat population genetic studies at the regional scale, there have also been a few studies at more narrow geographic scales highlighting some potential barriers to gene flow. A study completed in California used microsatellites to highlight the movement of bobcats in relation to barriers across an urban landscape, found significant genetic distance when the I-5 highway was considered (Lee et al. 2013). Two other population genetics studies conducted in California also reached the same conclusion regarding major highways as significant barriers to gene flow in bobcats (Ruell et al. 2012, Serieys et al. 2015).

In contrast, a study in Michigan found no impact of roads on bobcat gene flow, but significant genetic structure was observed between the bobcats of the Upper versus Lower Peninsula of that state (Millions and Swanson 2007). Reding et al. (2013) used microsatellite genotyping and mtDNA haplotyping in Oregon to identify significant genetic differentiations in 
bobcats located on opposite sides of the Cascade Mountain Range. These studies allude to landscape features as important factors having an impact on bobcat gene flow at a regional level.

Other studies have found little genetic differentiation among populations. Reid (2006) used six microsatellite loci to investigate the genetic differences between four bobcat populations located in Georgia and Florida. Little genetic differentiation was observed between these four populations, suggesting panmixia of bobcats in these areas. No evidence of population structure was observed in southern Illinois (Croteau et al. 2010). These studies, along with those previously mentioned, suggest that at the local scale, unless a significant barrier is present, bobcat gene flow is frequent in an interconnected landscape.

Reding et al. (2013) completed one of the few bobcat studies to explicitly use landscape genetics and radio telemetry in an attempt to quantify the impact of landscape on gene flow. The authors fitted 23 bobcats with radio collars to collect movement and habitat association data. These data were then combined with microsatellite genotypes from 625 individuals to calculate the least-cost paths of movement in the area. Bobcats were found to be most associated with forested habitats that were adjacent to grasslands. In conclusion, Reding et al. (2013) failed to reject the isolation by distance hypothesis for explaining genetic variation on the landscape, which means that even though severely fragmented, the Iowa landscape was not impeding gene flow in bobcats.

\section{Mark-Recapture}

Mark-recapture, also known as capture-recapture, is a specialized technique that can be used in wildlife management to estimate the size or density of a population in a specified area. Advancements in model construction followed by new computational technologies allowed for 
the rapid evolution of this technique during the 1960s, 1970s, and 1980s (Amstrup et al. 2010). The models used for mark-recapture studies are traditionally broken into one of two categories: closed-population models and open population models. Use of the appropriate model is important to the study design since these models have certain assumptions that must be met during sample collection. Closed-population models assume that a population is closed to outside influence during the study period. This means that there are no deaths, no immigration, no emigration, and no births while samples are being collected. While these assumptions can be easily violated, a study of short duration can effectively meet these assumptions (Amstrup et al., 2010). Open-population models allow for movement of individuals outside of the study area, as well as allow for deaths, births, and immigration; however, these models require additional covariates that can make analysis cumbersome.

Historically, mark-recapture has been utilized as a bobcat census technique in some states using either photographic or DNA fingerprinting to identify individuals. Heilbrun et al. (2006) used automated camera systems to document the 15 bobcats on a 3,156-ha refuge in Texas. Ruell et al. (2009) used bobcat scats to calculate bobcat densities of 0.25 and 0.42 bobcats $/ \mathrm{km}^{2}$ for two locations in the Santa Monica mountains of California. Larrucea et al. (2007) used automated camera arrays in Northern California to determine bobcat densities ranging from $0.27-0.39$ bobcats $/ \mathrm{km}^{2}$. A recent study that used single capture modified cable hair snares in Michigan's Upper Peninsula, found that the study area of $278.5 \mathrm{~km}^{2}$ contained a bobcat density of roughly 0.03 bobcats $/ \mathrm{km}^{2}$ (Stricker et al. 2012). Most recently Clare et al. (2015) used nine camera grids and a spatially explicit capture-recapture (SECR) study design to construct a model, which found an overall bobcat abundance of 362 individuals in a $14,286 \mathrm{~km}^{2}$ study area in Wisconsin. This study was the first application of SECR to bobcat research. 
Spatially explicit capture-recapture is a newly developing field that combines the population estimation capabilities of mark-recapture studies while accounting for the spatial variation of the landscape and trap placement that may impact the sampling process. Since the historic capture-recapture models are non-spatial, it is thought that a significant amount of error is introduced into the density estimation process by ignoring the spatial aspects of data collection (Royle et al. 2013). SECR was developed to deal with these deficiencies in capture-recapture sampling.

Some of the earlier SECR models were likelihood-based and accounted for the fact that animals with home ranges located closer to the sampling sites are more likely to be sampled than those that are further away (Borchers and Efford 2008). The SECR models also account for individual heterogeneity in being sampled, as well as the landscape variables at each site that may influence capture events (Royle et al. 2013).

More recent SECR models also model the least-cost path of animals from their home range centers to the sampling sites, allowing for a SECR model to not only determine density, but also landscape connectivity (Royle et al. 2013). A recent simulation study has shown that SECR outperforms other methods of density estimations such as mean maximum distance moved (MMDM) and an estimator using telemetry information, especially in situations where detection probabilities are low (Ivan et al. 2013), which is the case with elusive carnivores, like bobcats.

Since the publication of Clare et al. (2015), most studies seeking to quantify the density of bobcats in a particular study area have used SECR models. A camera trapping mark-recapture study using SECR found that bobcat capture probabilities were positively correlated with forest cover located in a buffer around camera traps and that estimated density was lowest nearby to heavily developed areas (Thornton and Pekins 2014), estimating an average density of 13.2 
bobcats per $100 \mathrm{~km}^{2}$ across the study sites. In the Appalachian Mountains of Virginia, on the border of West Virginia, Morin et al. (2018) used multiple, single-sample scat transects and multistrata SECR to estimate the local bobcat population density, finding estimates ranging from 5.93 bobcats / $100 \mathrm{~km}^{2}$ to 20.27 bobcats / $100 \mathrm{~km}^{2}$, with summer density estimates lower than those in winter.

\section{Occupancy Modeling and the Occupancy—Density Relationship}

Knowledge of the spatial distribution of a species is important for wildlife management. However, this information can be difficult to collect, especially for elusive carnivores like bobcats. When a site is surveyed for a particular species, it is possible that failure to collect data is the outcome of two possible conditions: 1) the species is not located in the survey area or 2) the survey method employed has an imperfect rate of detection $(<1)$ and failed to detect the species of interest that was in the survey area. Due to these two potential outcomes, a negative result in a species distribution survey cannot be assumed to indicate the absence of that species in a particular location, unless the detection method is perfect. None of the common methods used to detect bobcats at field sites (e.g., camera trapping, hair snares, and scat transects) has a perfect rate of detection.

To account for the issues associated with imperfect detection, MacKenzie et al. (2002) introduced a modeling framework that uses a likelihood-based method to estimate the occupancy of sampled and unsampled sites. The basic occupancy model has two parameters that are estimated for each species that is sampled. The first is the probability of detection, which is represented by $p$, and can range from $0-1$. The simplest definition of $p$ is the probability that, 
given the species of interest is located at a sampling site, it would be successfully sampled by the employed method. Since most detection methods are imperfect, and animals move across the landscape during a sampling period, $p$ is assumed to be less than 1 . The value of $p$ is estimated based on a maximum likelihood estimator calculated from the locations of detections combined with site and sampling covariates, such as temperature, cover type, and time of day (MacKenzie et al. 2002). The second parameter that is estimated is the probability of occupancy, which is represented by $\psi$ (psi). This value is the probability that a given sampling site is occupied by the species of interest. $\psi$ is also calculated using a maximum likelihood framework and can be related to site covariates that can be used to elucidate the ecology of the species being sampled (MacKenzie et al. 2002).

While the exact methods employed have changed over the years, the goal of occupancy modeling remains the same- to use detection and non-detection data in combination with habitat, time, and environmental covariates to predict species occupancy at the landscape scale. Since occupancy modeling only requires detection-nondetection data, it is more cost effective than traditional capture-recapture studies at examining species distributions (MacKenzie et al. 2002). Another advantage of occupancy modeling is that the extrapolation of the model across areas that were not sampled provides crucial data about these areas without incurring the costs of additional surveys. Additionally, occupancy modeling can be used to evaluate habitat selection based on how various habitat covariates of sampling sites create disparate rates of occupancy.

Long et al. (2011) sampled bobcats along with fishers (Martes pennanti) and American black bears (Ursus americanus) throughout the state of Vermont using non-invasive techniques. Bobcats were only detected at $11.9 \%$ of the total sites, for a model-averaged mean predicted occupancy of 32.6\% when taking into account the probability of detection. However, many of 
these sites were only visited once, against the recommendations of Mackenzie and Royle (2005) for situations when detection rates and occupancy are low. They identified two variables that had an impact on bobcat occupancy. The first variable was the percentage of the landscape in mixed forest located within $1 \mathrm{~km}$ of the study site and the second was the percentage of the landscape in forested wetlands also located within $1 \mathrm{~km}$ of the study site. While these variables were found to impact bobcat occupancy on the landscape, the size of their coefficients indicated that this impact was minimal. In their conclusion, the authors argued that the lack of a clear best model for this dataset is most likely the result of a less than ideal sampling design for elusive carnivores like bobcats.

Another important bobcat occupancy modeling study was conducted in central Wisconsin by Clare et al. (2015). This study also incorporated spatially-explicit capture recapture (SECR) into the occupancy model in order to predict bobcat abundance at the landscape scale ( $>14,000 \mathrm{~km})$ by using occupancy as a density index. Over the course of 13,595 camera trap nights at a total of 316 camera sites, a total of 250 bobcat detections were recorded. Occupancy was found to be positively correlated with the amount of woody cover on the landscape as well as the amount of wetland edge near the camera site. Bobcat detection was found to be positively correlated with dirt roads, nearby wetlands, and natural funneling features and negatively associated with human activity.

Erb et al. (2012) constructed an occupancy model for bobcat distribution along the Appalachian Trail corridor in North Carolina through Pennsylvania. Out of the 447 sampled sites, 43 (9.8\%) successfully detected bobcats. The final averaged model had four covariates that were associated with bobcat occupancy: percentage of forest cover within $10 \mathrm{~km}$ of the site, level of nearby hunting, usage intensity of nearby trail, and distance to roads. Bobcat occupancy was 
found to be positively correlated with the percentage of forest land cover and the distance to the nearest roads. In contrast, bobcat occupancy was determined to be negatively correlated with intensive nearby hunting as well as heavy trail use by humans. Wang et al. (2015) investigated the mesocarnivore responses to pumas and human development in the Santa Cruz Mountains of California and found that as human development densities increased, bobcat detection and occupancy probabilities dropped. Lewis et al. (2015) reached a similar conclusion after constructing occupancy models for two sites in Colorado.

Lesmeister et al. (2015) used camera trap grids in southern Illinois to investigate the carnivore guilds of that area. At the local level of the sites, the authors found that habitat variables had very little explanatory power for bobcat occupancy and asserted that bobcat habitat use at the fine scale was most likely random. However, at the landscape scale, bobcat occupancy was again found to be negatively correlated with the intensity of human development, as well as paved road density, and the presence of structures. The authors concluded that in their research areas bobcats had a high overall predicted occupancy $(0.5-1.0)$, while the average probability of detection was low ( 0.20 per week), as was expected for this type of wide-ranging mesocarnivore.

The utility of presence-absence data is not limited to just determining the distribution of a particular species. Royle and Nichols (2003) developed a model where the abundance of a species could be estimated based on the location of detections and non-detections, after repeated surveys at the same sites. The bases of this model are the relationship between abundance and detection probability, and variation in detection as correlated to variance in abundance at individual sites. The authors found little evidence of bias or error in their abundance estimates when a large number of sites were used in the overall study. 
An application of this model to another low-density carnivore species, the fisher, found that population estimates derived from an SECR model were very similar to those obtained by fitting a Royle-Nichols model to the same dataset (Linden et al. 2017). The purpose of this research was to study fisher occupancy and abundance throughout a study area similar to the size of West Virginia $\left(70,069 \mathrm{~km}^{2}\right)$. The authors asserted that when the size of spatial grain for each sampling site was such that only one individual was assumed to be occupying each site, that a clear relationship between occupancy and abundance could be modeled. This study operated under a similar provision as had been used by Clare et al. (2015), where SECR was used to identify the point pattern of individuals. This pattern was then applied to determine the relationship between occupancy and density and used to project the estimated abundance across a larger study area, the entirety of which was not sampled.

\section{Bobcat Management in West Virginia}

The current bobcat population model used for management in West Virginia was adapted from Crowe (1975), which was originally constructed for exploited bobcat populations in Wyoming. This model predicts the yearly change in population size per year based on the survival rates of adults, the survival rates of the young, and the number of female young produced per female. The adult and juvenile survival rates for West Virginia bobcats, as well as the number of female young per female, were published by Fox and Fox (1982). However, this model has no power for determining the change in the population size without baseline population data, which to this point has been made available using one of two methods. The first is density estimates for Virginia in areas with similar habitats to West Virginia (Progulske, 1952, McCord and Cordoza, 1982). The second method is to use harvest data; however, harvest effort 
can bias data due to extreme weather events or fur price fluctuations both of which influence participation rate and effort (personal communication: Rich Rogers, WVDNR). Therefore, the current population model is based on assumptions which may no longer apply to the current population.

\section{Study Objectives}

At the state level, little data exist on bobcat gene flow and migration. At the level of local spatial scale in the mid-Atlantic region, very little research has been completed on bobcats using genetic methods to investigate population structure and dispersal. Due to the lack of knowledge on population genetic structure of bobcats in West Virginia and surrounding states, research on how genes flow in these animals was undertaken along with determining the size and density of the bobcat population. Also, no detailed population studies have been completed to date in West Virginia and the current population model used in making management decisions (Fox and Fox, 1982) is more than 30 years old — and needs to be reexamined for validation. The objectives and hypotheses of this study are listed in their relevant chapters listed below.

\section{Dissertation Chapter 2: Bobcat Hair Snare Cubby}

While most recent non-invasive bobcat studies have made use of camera surveys, the prohibitive cost of camera stations limits the overall size of area that can be sampled. One of the primary of this study was to evaluate the distribution and relative abundance of bobcats throughout the state of West Virginia. In order to make this possible, a new hair snare needed to be designed that would meet the unique needs of the West Virginia landscape. 


\section{Research Objectives:}

1. Design a new and effective hair snare for bobcats that meets the following criteria:

a. Able to be deployed regardless of terrain features;

b. Does not require a rubbing behavioral response to collect samples;

c. Easy to transport, deploy, and check;

d. Durable enough for long-term use (6+ months deployed continuously); and

e. Reliable in collecting hair samples from individual bobcats.

2. Evaluate the success of the new hair snare in terms of detections per 100 trap nights and compare to previous studies.

3. Provide recommendations to researchers looking to deploy this new hair snare based on knowledge gained from this study.

\section{Dissertation Chapter 3: Landscape-Scale Occupancy Model of the Bobcats of West Virginia}

Due to the relatively recent increase in bobcat populations from historical lows (Roberts and Crimmins, 2010), it is likely that there are areas within West Virginia and bordering states that currently do not have breeding bobcat populations. While habitat may be available, these areas are capable of being colonized by juveniles and yearlings from population sources (Kitchings and Story 1984, Croteau et al., 2010). Detection-nondetection data can be valuable in determining the distribution of elusive carnivore species, as well as particular landscape variables that determine bobcat location preference on a continuous landscape. While bobcats are harvested in every county of West Virginia, there are locations with acceptable habitat that have been historically devoid of sightings, sign, or harvest. Detection-nondetection data collected 
during the capture-recapture portion of this project will be used to evaluate the occupancy of bobcats at the landscape scale in West Virginia.

\section{Research Objectives:}

1. Construct a statewide occupancy model for bobcats in West Virginia.

2. Determine which environmental, landscape, and sampling variables impact bobcat detection probability or occupancy.

\section{Dissertation Chapter 4: Abundance and Density Estimates of West Virginia Bobcats}

Density estimates of bobcats in West Virginia have never been evaluated in a formal study. To assist the WVDNR in ensuring continued, sustainable bobcat harvests, abundance and density estimates for the bobcat population in West Virginia will be determined. This will be achieved using a combination of spatially-explicit capture-recapture and occupancy modeling.

\section{Research Objectives:}

1. Use spatially-explicit capture-recapture (SECR) (Borchers and Efford 2008) and occupancy modeling to estimate the abundance and density of bobcats across the study area in West Virginia. This is to be completed for the whole state, as well as each ecological region as described by Uhlig and Wilson (1952).

2. Compare abundance and density estimates between occupancy Royle-Nichols models (Royle and Nichols 2003) and SECR (Borchers and Efford 2008).

3. Compare and contrast the estimated population abundances and densities to those calculated using West Virginia’s current bobcat population model. 
4. Determine if a new population growth model would better represent West Virginia's bobcat population than what is currently used for management.

\section{Dissertation Chapter 5: Population and Genetics of West Virginia Bobcats}

The current population of bobcats in at least some of the six ecological regions of West Virginia (Uhlig and Wilson 1952) are suspected to have some definable population structure. While some studies have found panmixia in bobcats across small geographic scales (Reid 2006, Croteau et al. 2010), two studies involving the use of a roadway barrier found discernable population structure in California (Riley et al. 2006, Ruell et al. 2012, Serieys et al. 2015). However, another study conducted in Michigan found that roads were not a factor in the population structure of bobcats (Millions and Swanson 2007). While the majority of West Virginia is forested, human impacts may reduce geneflow and result in population structure. In addition, the population reduction of bobcats witnessed across the United States between the 1930s until the 1980s (Roberts and Crimmins 2010) is thought to have caused a genetic bottleneck in the bobcats of West Virginia. Since the population was at a low level for a period of roughly 20 years, genetic drift may have been a factor in the loss of alleles, which has the most pronounced impact in small, isolated populations (Allendorf 1986).

\section{Research Objectives:}

1. Determine whether or not population structure is present in the bobcats of West Virginia or if they are best characterized by panmixia.

2. Determine the effective population size $\left(\mathrm{N}_{\mathrm{e}}\right)$ of the bobcats of West Virginia and, if enough data are available, the $\mathrm{N}_{\mathrm{e}}$ for each identified population. 
3. Investigate whether or not barriers to gene flow are present on the landscape.

4. Determine whether bobcat populations in West Virginia were subjected to a recent genetic bottleneck.

5. In regard to maintaining gene flow, determine whether bobcats should be managed as smaller, distinct populations within West Virginia.

Dissertation Chapter 6: Parvovirus Infection Prevalence and Distribution Analysis of Bobcats of West Virginia

The extent to which Feline Panleukopenia Virus (FPV) or Canine Parvovirus (CPV) is having an impact on the bobcat populations in West Virginia is unknown. While it is suspected that domestic felids are a reservoir for these viruses on the landscape, this relationship has not yet been investigated. Molecular techniques will be used to evaluate necropsied bobcats for the presence of FPV or CPV infections and these data will be correlated to landscape variables to determine if any spatial patterns of infection exist.

\section{Research Objectives:}

1. Use molecular methods to investigate bobcat spleen samples for the presence of parvovirus DNA and determine overall statewide prevalence rate.

2. Sequence DNA from parvovirus positive samples to determine type of parvovirus infecting each bobcat found to be positive.

3. Use GIS techniques to determine if prevalence of bobcats with parvovirus infections is greater than would be expected by random chance in more urbanized counties of West Virginia. 
4. Investigate the presence of potential geographical patterns in different types of parvoviruses found in West Virginia's bobcats. 


\section{References}

Allendorf, F. W. 1986. Genetic Drift and the loss of Alleles Versus Heterozygosity. Zoo Biology 5:181-190.

Allison, A. B., C. E. Harbison, I. Pagan, K. M. Stucker, J. T. Kaelber, J. D. Brown, M. G. Ruder, M. K. Keel, E. J. Dubovi, E. C. Holmes, and C. R. Parrish. 2012. Role of Multiple Hosts in the Cross-Species Transmission and Emergence of a Pandemic Parvovirus. Journal of Virology 86:865-872.

Allison, A. B., D. J. Kohler, K. A. Fox, J. D. Brown, R. W. Gerhold, V. I. Shearn-Bochsler, E. J. Dubovi, C. R. Parrish, and E. C. Holmes. 2013. Frequent Cross-Species Transmission of Parvoviruses among Diverse Carnivore Hosts. Journal of Virology 87:2342-2347.

Allison, A. B., D. J. Kohler, A. Ortega, E. A. Hoover, D. M. Grove, E. C. Holmes, and C. R. Parrish. 2014. Host-Specific Parvovirus Evolution in Nature Is Recapitulated by In Vitro Adaptation to Different Carnivore Species. PLOS Pathogens 10:e1004475.

Amstrup, S. C., T. L. McDonald, and B. F. J. Manly, editors. 2010. Handbook of CaptureRecapture Analysis. Princeton University Press.

Anderson, C. S., S. Prange, and H. L. Gibbs. 2015. Origin and Genetic Structure of a Recovering Bobcat (Lynx rufus) Population. Canadian Journal of Zoology 93:889-899.

Bailey, T. N. 1974. Social Organization in a Bobcat Population. The Journal of Wildlife Management 38:435.

Battilani, M., L. Gallina, F. Vaccari, and L. Morganti. 2007. Co-Infection with Multiple Variants of Canine Parvovirus Type 2 (CPV-2). Veterinary Research Communications; Dordrecht 31:209-12.

Benson, J. F., M. J. Chamberlain, and B. D. Leopold. 2004. Land Tenure and Occupation of Vacant Home Ranges by Bobcats (Lynx rufus). Journal of Mammalogy 85:983-988.

Borchers, D. L., and M. G. Efford. 2008. Spatially Explicit Maximum Likelihood Methods for Capture-Recapture Studies. Biometrics 64:377-385.

Clare, J. D. J., E. M. Anderson, and D. M. MacFarland. 2015. Predicting Bobcat Abundance at a Landscape Scale and Evaluating Occupancy as a Density Index in Central Wisconsin. The Journal of Wildlife Management 79:469-480.

Clegg, S. R., K. P. Coyne, S. Dawson, N. Spibey, R. M. Gaskell, and A. D. Radford. 2012. Canine Parvovirus in Asymptomatic Feline Carriers. Veterinary Microbiology 157:78-85.

Comer, C. E., M. E. Symmank, and J. C. Kroll. 2011. Bobcats Exhibit Low Detection Rates at Hair Collection Stations in East Texas. Wildlife Biology in Practice 7(1):116-122. 
Cotmore, S. F., M. Agbandje-McKenna, J. A. Chiorini, D. V. Mukha, D. J. Pintel, J. Qiu, M. Soderlund-Venermo, P. Tattersall, P. Tijssen, D. Gatherer, and A. J. Davison. 2014. The Family Parvoviridae. Archives of Virology 159:1239-1247.

Convention on International Trade in Endangered Species of Wild Fauna and Flora (2010). Fifteenth meeting of the Conference of the Parties (2010). Conference of Parties Proposal II.

Croteau, E. K., E. J. Heist, and C. K. Nielsen. 2010. Fine-scale Population Structure and SexBiased Dispersal in Bobcats (Lynx rufus) from Southern Illinois. Canadian Journal of Zoology 88:536-545.

Croteau, E. K., E. J. Heist, C. K. Nielsen, J. R. Hutchinson, and E. C. Hellgren. 2012.

Microsatellites and Mitochondrial DNA Reveal Regional Population Structure in Bobcats (Lynx rufus) of North America. Conservation Genetics 13:1637-1651.

Crowe, D. M. 1975. A Model for Exploited Bobcat Populations in Wyoming. The Journal of Wildlife Management 39:408.

Crowley, S. M., and D. P. Hodder. 2017. An Assessment of the Efficacy of Rub Stations for Detection and Abundance Surveys of Canada lynx (Lynx canadensis). Canadian Journal of Zoology 95:885-890.

Csiza, C. K., A. D. Lahunta, F. W. Scott, and J. H. Gillespie. 1971. Pathogenesis of Feline Panleukopenia Virus in Susceptible Newborn Kittens II. Pathology and Immunofluorescence. Infection and Immunity 3:838-846.

Decaro, N., D. Buonavoglia, C. Desario, F. Amorisco, M. L. Colaianni, A. Parisi, V. Terio, G. Elia, M. S. Lucente, A. Cavalli, V. Martella, and C. Buonavoglia. 2010. Characterisation of Canine Parvovirus Strains Isolated from Cats with Feline Panleukopenia. Research in Veterinary Science 89:275-278.

Depue, J. E., and M. Ben-David. 2007. Hair Sampling Techniques for River Otters. The Journal of Wildlife Management 71:671-674.

Elizalde-Arellano, C., J. C. López-Vidal, L. Hernández, J. W. Laundré, F. A. Cervantes, and M. Alonso-Spilsbury. 2012. Home Range Size and Activity Patterns of Bobcats (Lynx rufus) in the Southern Part of Their Range in the Chihuahuan Desert, Mexico. The American Midland Naturalist 168:247-264.

Erb, P. L., W. J. McShea, and R. P. Guralnick. 2012. Anthropogenic Influences on Macro-Level Mammal Occupancy in the Appalachian Trail Corridor. PLOS ONE 7:e42574.

Foley, J. E., P. Swift, K. A. Fleer, S. Torres, Y. A. Girard, and C. K. Johnson. 2013. Risk Factors for Exposure to Feline Pathogens in California Mountain Lions (Puma concolor). Journal of Wildlife Diseases 49:279-293. 
Fox, L. B., and J. S. Fox. 1982. Population Characteristics and Food Habits of Bobcats in West Virginia. Proceedings of the Annual Conference of the Southeast Association of Fish and Wildlife Agencies.

Harrison, R. L. 2010. Ecological Relationships of Bobcats (Lynx rufus) in the Chihuahuan Desert of New Mexico. The Southwestern Naturalist 55:374-381.

Heilbrun, R. D., N. J. Silvy, M. J. Peterson, and M. E. Tewes. 2006. Estimating bobcat abundance using automatically triggered cameras. Wildlife Society Bulletin 34:69-73.

Heilbrun, R. D., N. J. Silvy, M. E. Tewes, and M. J. Peterson. 2003. Using Automatically Triggered Cameras to Individually Identify Bobcats. Wildlife Society Bulletin 31:748755.

Hines J.E. (2006) PRESENCE-Software to Estimate Patch Occupancy and Related Parameters. Available: http://www.mbr-pwrc.usgs.gov/software/presence.html.

Hoelzer, K., L. A. Shackelton, C. R. Parrish, and E. C. Holmes. 2008. Phylogenetic Analysis Reveals the Emergence, Evolution and Dispersal of Carnivore Parvoviruses. Journal of General Virology 89:2280-2289.

Horiuchi, M., K. Yuri, T. Soma, H. Katae, H. Nagasawa, and M. Shinagawa. 1996. Differentiation of Vaccine Virus from Field Isolates of Feline Panleukopenia Virus by Polymerase Chain Reaction and Restriction Fragment Length Polymorphism Analysis. Veterinary Microbiology 53:283-293.

Ikeda, Y., M. Mochizuki, R. Naito, K. Nakamura, T. Miyazawa, T. Mikami, and E. Takahashi. 2000. Predominance of Canine Parvovirus (CPV) in Unvaccinated Cat Populations and Emergence of New Antigenic Types of CPVs in Cats. Virology 278:13-19.

Ivan J. S., G. C. White, and T.M. Shenk. 2013. Using simulation to compare methods for estimating density from capture-recapture data. Ecology 94:817-826.

Janečka, J. E., T. L. Blankenship, D. H. Hirth, M. E. Tewes, C. W. Kilpatrick, and L. I. Grassman. 2006. Kinship and Social Structure of Bobcats (Lynx rufus) Inferred from Microsatellite and Radio-telemetry Data. Journal of Zoology 269:494-501.

Janečka, J. E., T. L. Blankenship, D. H. Hirth, C. William Kilpatrick, M. E. Tewes, and L. I. Grassman. 2007. Evidence for Male-biased Dispersal in Bobcats Lynx Rufus Using Relatedness Analysis. Wildlife Biology 13:38-47.

Johnson, S. A., H. D. Walker, and C. M. Hudson. 2010. Dispersal Characteristics of Juvenile Bobcats in South-Central Indiana. Journal of Wildlife Management 74:379-385.

Kitchings, J. T., and J. D. Story. 1984. Movements and Dispersal of Bobcats in East Tennessee. The Journal of Wildlife Management 48:957. 
Kruse, B. D., S. Unterer, K. Horlacher, C. Sauter-Louis, and K. Hartmann. 2010. Prognostic Factors in Cats with Feline Panleukopenia. Journal of Veterinary Internal Medicine 24:1271-1276.

Laake, J. 2013. RMark: An R Interface for Analysis of Capture-Recapture Data with MARK. AFSC Processed Report 2013-01.

Landry, Stephanie Michelle. 2017. Bobcat Population Ecology in West Virginia. West Virginia University. Ann Arbor: ProQuest.

Lane, E. P., H. Brettschneider, P. Caldwell, A. Oosthuizen, D. L. Dalton, L. du Plessis, J. Steyl, and A. Kotze. 2016. Feline Panleukopaenia Virus in Captive Non-domestic Felids in South Africa. Onderstepoort Journal of Veterinary Research 83:1-8.

Larrucea, E. S., G. Serra, M. M. Jaeger, and R. H. Barrett. 2007. Censusing Bobcats Using Remote Cameras. Western North American Naturalist 67:538-548.

Lee, J. S., E. W. Ruell, E. E. Boydston, L. M. Lyren, R. S. Alonso, J. L. Troyer, K. R. Crooks, and S. VandeWoude. 2012. Gene flow and Pathogen Transmission Among Bobcats (Lynx rufus) in a Fragmented Urban Landscape. Molecular Ecology 21:1617-1631.

Lesmeister, D. B., C. K. Nielsen, E. M. Schauber, and E. C. Hellgren. 2015. Spatial and Temporal Structure of a Mesocarnivore Guild in Midwestern North America. Wildlife Monographs 191:1-61.

Lewis, J. S., K. A. Logan, M. W. Alldredge, L. L. Bailey, S. VandeWoude, and K. R. Crooks. 2015. The Effects of Urbanization on Population Density, Occupancy, and Detection Probability of Wild Felids. Ecological Applications 25:1880-1895.

Linden, D. W., A. K. Fuller, J. A. Royle, and M. P. Hare. 2017. Examining the OccupancyDensity Relationship for a Low-density Carnivore. Journal of Applied Ecology 54:20432052.

Litvaitis, J. A., J. P. Tash, and C. L. Stevens. 2006. The Rise and Fall of Bobcat Populations in New Hampshire: Relevance of Historical Harvests to Understanding Current Patterns of Abundance and Distribution. Biological Conservation 128:517-528.

Lombardi, J. V., C. E. Comer, D. G. Scognamillo, and W. C. Conway. 2017. Coyote, Fox, and Bobcat Response to Anthropogenic and Natural Landscape Features in a Small Urban Area. Urban Ecosystems 20:1239-1248.

Long, R. A., T. M. Donovan, P. Mackay, W. J. Zielinski, and J. S. Buzas. 2007. Comparing Scat Detection Dogs, Cameras, and Hair Snares for Surveying Carnivores. The Journal of Wildlife Management 71:2018-2025.

Long, R. A., T. M. Donovan, P. MacKay, W. J. Zielinski, and J. S. Buzas. 2011. Predicting Carnivore Occurrence with Noninvasive Surveys and Occupancy Modeling. Landscape Ecology 26:327-340. 
Lovallo, M. J., and E. M. Anderson. 1995. Range Shift by a Female Bobcat (Lynx rufus) After Removal of Neighboring Female. American Midland Naturalist 134:409.

MacKenzie, D. I., and L. L. Bailey. 2004. Assessing the fit of site-occupancy models. Journal of Agricultural, Biological, and Environmental Statistics 9:300-318.

MacKenzie, D. I., J. D. Nichols, G. B. Lachman, S. Droege, J. A. Royle, and C. A. Langtimm. 2002. Estimating Site Occupancy Rates When Detection Probabilities Are Less Than One. Ecology 83:2248-2255.

Mackenzie, D. I., and J. A. Royle. 2005. Designing Occupancy Studies: General Advice and Allocating Survey Effort. Journal of Applied Ecology 42:1105-1114.

MacKenzie, D. I., L. L. Bailey, J. E. Hines, J. D. Nichols, K. H. Pollock, and J. A. Royle. 2005. Occupancy Estimation and Modeling: Inferring Patterns and Dynamics of Species Occurrence. Elsevier Science \& Technology, San Diego, United States.

Manel, S., M. K. Schwartz, G. Luikart, and P. Taberlet. 2003. Landscape Genetics: Combining Landscape Ecology and Population Genetics. Trends in Ecology \& Evolution 18:189197.

Maryland Department of Natural Resources (2014). Wildlife Heritage Service. Furbearers of Maryland - The Game Program.

McCord, C.M. and J.E. Cardoza. 1982. Bobcat and Lynx. Pages 728-766 in J.A. Chapman and G.A. Feldhammer, eds. Wild mammals of North America: Biology, Management, and Economics. Johns Hopkins Univ. Press, Baltimore, Md.

McDaniel, G. W., K. S. McKelvey, J. R. Squires, and L. F. Ruggiero. 2000. Efficacy of Lures and Hair Snares to Detect Lynx. Wildlife Society Bulletin 28:119-123.

Millions, D. G., and B. J. Swanson. 2007. Impact of Natural and Artificial Barriers to Dispersal on the Population Structure of Bobcats. Journal of Wildlife Management 71:96-102.

Miranda, C., C. R. Parrish, and G. Thompson. 2014. Canine Parvovirus 2c Infection in a Cat with Severe Clinical Disease. Journal of Veterinary Diagnostic Investigation 26:462-464.

Morin, D. J., L. P. Waits, D. C. McNitt, and M. J. Kelly. 2018. Efficient Single-survey Estimation of Carnivore Density Using Fecal DNA and Spatial Capture-recapture: A Bobcat Case Study. Population Ecology 60(3):197-209.

Nogeire, T. M., F. W. Davis, J. M. Duggan, K. R. Crooks, and E. E. Boydston. 2013. Carnivore Use of Avocado Orchards across an Agricultural-Wildland Gradient. PLOS ONE 8:e68025.

Ohio Bobcat Management Plan. 2018. Ohio Department of Natural Resources Division of Wildlife. Columbus, $\mathrm{OH}$. 
Ohio Department of Natural Resources. 2014. Biologists Propose Removing Bobcats from Ohio’s Threatened Species List.

Progulske, D. R. 1952. The bobcat and its relation to prey species in Virginia. M.S. Thesis. Virginia Polytechnic Institute, 135 pp.

Ray, J. C. 2000. Mesocarnivores of northeastern North America: status and conservation issues. Wildlife Conservation Society Working Paper No. 15.

Reding, D. M., A. M. Bronikowski, W. E. Johnson, and W. R. Clark. 2012. Pleistocene and Ecological Effects on Continental-scale Genetic Differentiation in the Bobcat (Lynx rufus). Molecular Ecology 21:3078-3093.

Reding, D. M., S. A. Cushman, T. E. Gosselink, and W. R. Clark. 2013. Linking Movement Behavior and Fine-scale Genetic Structure to Model Landscape Connectivity for Bobcats (Lynx rufus). Landscape Ecology 28:471-486.

Reed, G. C. 2013. Bobcats in New Hampshire: Understanding the Relationships Between Habitat Suitability, Connectivity and Abundance in a Changing Landscape. M.S. Thesis. University of New Hampshire, 126 pp.

Reid, A. E. 2006. Spatial Genetic Structure of Four Bobcat Populations in the Southeastern US. M.S. Thesis. University of Georgia, 110 pp.

Riley, S. P. D., J. P. Pollinger, R. M. Sauvajot, E. C. York, C. Bromley, T. K. Fuller, and R. K. Wayne. 2006. FAST-TRACK: A Southern California Freeway is a Physical and Social Barrier to Gene Flow in Carnivores. Molecular Ecology 15:1733-1741.

Roberts, N. M., and S. M. Crimmins. 2010. Bobcat Population Status and Management in North America: Evidence of Large-Scale Population Increase. Journal of Fish and Wildlife Management 1:169-174.

Royle, J. A., R. B. Chandler, R. Sollmann, and B. Gardner. 2013. Spatial Capture-Recapture. Academic Press.

Royle J. A., R. B. Chandler, K. D. Gazenski, and T.A. Graves. 2013. Spatial Capture-recapture Models for Jointly Estimating Population Density and Landscape Connectivity. Ecology 94:287-294.

Royle J. A., and J.D. Nichols. 2003. Estimating abundance from repeated presence-absence data or point counts. Ecology 84:777-790.

Ruell, E. W., S. P. D. Riley, M. R. Douglas, M. F. Antolin, J. R. Pollinger, J. A. Tracey, L. M. Lyren, E. E. Boydston, R. N. Fisher, and K. R. Crooks. 2012. Urban Habitat Fragmentation and Genetic Population Structure of Bobcats in Coastal Southern California. The American Midland Naturalist 168:265-280. 
Ruell, E. W., S. P. D. Riley, M. R. Douglas, J. P. Pollinger, and K. R. Crooks. 2009. Estimating Bobcat Population Sizes and Densities in a Fragmented Urban Landscape Using Noninvasive Capture-Recapture Sampling. Journal of Mammalogy 90:129-135.

Schunck, B., W. Kraft, and U. Truyen. 1995. A Simple Touch-down Polymerase Chain Reaction for the Detection of Canine Parvovirus and Feline Panleukopenia Virus in Feces. Journal of Virological Methods 55:427-433.

Serieys, L. E. K., A. Lea, J. P. Pollinger, S. P. D. Riley, and R. K. Wayne. 2015. Disease and Freeways Drive Genetic Change in Urban Bobcat Populations. Evolutionary Applications 8:75-92.

Siegl, G., R. C. Bates, K. I. Berns, B. J. Carter, D. C. Kelly, E. Kurstak, and P. Tattersall. 1985. Characteristics and Taxonomy of Parvoviridae. Intervirology 23:61-73.

Storfer, A., M. A. Murphy, J. S. Evans, C. S. Goldberg, S. Robinson, S. F. Spear, R. Dezzani, E. Delmelle, L. Vierling, and L. P. Waits. 2007. Putting the 'Landscape' in Landscape Genetics. Heredity 98:128-142.

Stricker, H. K., J. L. Belant, D. E. Beyer, J. Kanefsky, K. T. Scribner, D. R. Etter, and J. Fierke. 2012. Use of Modified Snares to Estimate Bobcat Abundance. Wildlife Society Bulletin 36:257-263.

Symmank, M., C. Comer, and J. Kroll. 2008. Estimating Bobcat Abundance in East Texas Using Infrared-triggered Cameras. Proceedings of the Annual Conference of the Southeastern Association of Fish and Wildlife Agencies 62:64-69.

Thornton, D. H., and C. E. Pekins. 2015. Spatially Explicit Capture-recapture Analysis of Bobcat (Lynx rufus) Density: Implications for Mesocarnivore Monitoring. Wildlife Research 42:394.

Uhlig, H. G., and H. L. Wilson. 1952. A Method of Evaluating an Annual Mast Index. The Journal of Wildlife Management. 16:338-343.

Virginia Department of Game and Inland Fisheries (2014). Furbearer trapping seasons and general regulations.

Wang, Y., M. L. Allen, and C. C. Wilmers. 2015. Mesopredator Spatial and Temporal Responses to Large Predators and Human Development in the Santa Cruz Mountains of California. Biological Conservation 190:23-33.

Wasieri, J., G. Schmiedeknecht, C. Förster, M. König, and M. Reinacher. 2009. Parvovirus Infection in a Eurasian Lynx (Lynx lynx) and in a European Wildcat (Felis silvestris silvestris). Journal of Comparative Pathology 140:203-207.

Weaver, J. L., P. Wood, D. Paetkau, and L. L. Laack. 2005. Use of Scented Hair Snares to Detect Ocelots. Wildlife Society Bulletin 33:1384-1391. 
West Virginia Division of Natural Resources (2014). General 2014-2015 Hunting regulations.

White, G. C., and K. P. Burnham. 1999. Program MARK: Survival Estimation from Populations of Marked Animals. Bird Study 46:S120-S139. 


\title{
Chapter 2 - The Bobcat Hair Snare Cubby: A Non-Invasive Tool for Bobcat Population Monitoring
}

\begin{abstract}
Over the past 20 years, non-invasive hair snare surveys have become common tools used in wildlife research and management. While these tools have been used to answer important research questions on a number of species, these techniques often fail to gather information on elusive carnivores, such as bobcats (Lynx rufus). Due to the mixed success of previous bobcat studies using hair snares, this technique has largely fallen out of use for this species. The goal of this study was to construct a novel bobcat hair snare that could be deployed regardless of terrain or vegetation features which would be effective for use in capture-recapture population estimation at a large spatial scale. This new hair snare was deployed at 1,500 total sites across West Virginia between two sampling seasons (2015 - 2016). Collected hair samples were analyzed with newly developed mitochondrial DNA primers and qPCR to determine species of origin. Over the two years of the study a total of 378 bobcat detections were recorded from 42,000 trap nights of sampling, for an overall rate of 0.9 detections per 100 trap nights. This study marks the development of a potentially important new technique for use in bobcat management.
\end{abstract}

IACUC Approval \#: 14-1108 (appendix Document 1) 


\section{Introduction}

The bobcat (Lynx rufus) is a moderately sized felid that is primarily carnivorous with a lifecycle completely dependent on the availability and abundance of prey species, which impacts bobcat habitat selection (Chamberlain et al. 1999, 2003). Being generalist predators they quickly switch their diets to take advantage of periodic or season population increases of certain prey species, thus mitigating the environmental impacts of such (Beasom and Moore 1977). The bobcat has established populations in each of the contiguous United States except for Delaware, making it one of the most widely distributed carnivores in North America (Roberts and Crimmins 2010). In some states, bobcats are currently hunted and trapped for their valuable fur; however, in others they are considered a protected species due to either unknown or low overall abundance.

Since the 1980s bobcat abundance across the United States has increased dramatically. Roberts and Crimmins (2010) discovered this trend when comparing the results of a questionnaire survey they submitted to each of the state wildlife agencies of the contiguous 48 states, to a similar survey conducted by the U.S. Fish and Wildlife Service in 1981. More than $80 \%$ of respondents indicated that bobcat populations in their respective states were either increasing or stable. However, only about $50 \%$ of state agencies reported an estimated population size or density for their bobcat populations. Bobcats are not provided any protections under federal law, but their harvests are governed by the Convention on International Trade in Endangered Species of Wild Fauna and Flora (CITES) under Appendix II, due similarity in appearance of pelts to the endangered Iberian lynx (Lynx pardinus) (United States Fish and Wildlife Service 1982). To maintain compliance with CITES, any state that allows hunting or trapping of bobcats is required to demonstrate sustainability of these activities, which requires 
detailed population information. Since bobcats are an elusive carnivore species, the collection of these data can be a difficult task.

Camera traps, scat transects, examination of harvest data, and hair snares are the noninvasive methods most commonly used to collect bobcat population information. Camera traps have been successfully used to document bobcat presence and estimate abundance, but only when the unique spot patterns of individuals can be identified (Clare et al. 2015, Heilbrun et al. 2003, Heilbrun et al. 2006, Larrucea et al. 2007, Long et al. 2007, Symmank et al. 2008, Thornton and Pekins 2015). Camera trap costs have declined significantly since the first study conducted in 2003; however, using cameras to survey large areas still requires a sizable investment of financial and personnel resources due to the need to use paired cameras for individual identification. Scat transects, conducted using both human observers (Ruell et al. 2009, Morin et al. 2018) and detection dogs (Harrison 2006, Long et al. 2007), have also been successfully applied to the estimation of bobcat abundance or occupancy. When directly comparing these two methods, scat detection dogs were found to be the most effective at finding bobcat samples, but they also had the greatest associated costs (Harrison 2006, Long et al. 2007), which can greatly reduce the area that can be surveyed when resources are limited. In contrast, hair snares have the lowest deployment costs, allowing for a much larger area to be surveyed for the same total costs. For large-scale studies, hair snares have often been the only cost-effective option available, in spite of low success.

The use of hair snares in wild felid research came into prominence after the development of the carpet scratch pad by McDaniel et al. (2000), which was successful in collecting hair samples from Canada lynx (Lynx canadensis). This device was effectively used to study other species such as ocelot (Leopardus pardalis) (Weaver et al. 2005); however, little success was 
reported when using the same carpet scratch pads to specifically target bobcats. In New Mexico, Harrison (2006) installed the carpet scratch pads, as described in McDaniel et al. (2000), and achieved only one detection over 700 trap nights, even though bobcats were known to inhabit the study sites. Long et al. (2007) deployed these same devices in known bobcat habitat in Vermont for 2,072 trap nights and failed to achieve any detections. More recently, Comer et al. (2011) collected hair from only one bobcat in 1,680 trap nights in eastern Texas, even though bobcats were detected by cameras at 15 carpet scratch pad sites. The authors of these three studies reached the same conclusion: hair snares are not successful at non-invasively sampling wild bobcats. More recently, the efficacy of the original carpet scratch pads for Canada lynx research has also come into question due to the variability between individuals in cheek rubbing behaviors required for sampling (Crowley and Hodder 2017).

Due to the poor performance of the carpet scratch pads for bobcat research, a more recent study has utilized a new approach to attempt to collect bobcat hair. In the upper peninsula of Michigan, Stricker et al. (2012) deployed a modified cable snare (DePue and Ben-David 2007) that was designed as a single-sample device placed in areas surrounding sites baited with deer carcasses. This device was constructed specifically as a passive device to collect hair samples without the required cheek rubbing behavior of the carpet scratch pad. Over the course of an 8week study in a $278.5 \mathrm{~km}^{2}$ study area, a total of 230 hair samples were collected, of which 17 originated from bobcats. This study documented the successful use of hair snares to sample wild bobcats and estimate population size by using a novel method.

Our project was completed as a portion of a study seeking to estimate relative abundances, and potentially, densities of bobcats across West Virginia $\left(63,000 \mathrm{~km}^{2}\right)$. Hair snares were selected as the only non-invasive method that would be economically feasible to sample 
such a large area. While the technique used by Stricker et al. (2012) was successful in collecting hair samples from bobcats, the topographic complexity of West Virginia makes the use of this method logistically prohibitive due to absence of travel corridors between patches of forest, as compared to the Upper Peninsula of Michigan. The first goal of this study was to develop a new and effective hair snare that is able to meet the following criteria: 1) able to be deployed independent of terrain features; 2) does not require behavioral rubbing to collect samples; 3) easy to transport, deploy, and check; 4) was durable enough for long-term use (6+ months deployed continuously); and 5) reliably collects hair samples from individual bobcats. The second goal of the study was to evaluate the success of the new hair snare in terms of detections per 100 trap nights and compare to previous studies. Finally, the third goal was to provide recommendations for researchers wanting to deploy this hair snare based on the knowledge gained from this study.

\section{Materials and Methods}

\section{Hair Snare Cubby Design}

To meet the specific needs of sampling bobcats in the central Appalachian Mountains a new hair snare was developed using what was described by Kendall et al. (2008) for sampling fisher (Martes pennanti) populations as a starting point for modifications. Firstly, the overall height and length of the device, as described by Kendall et al. (2008), was increased to accommodate the larger stature of bobcats. A second modification was that mesh was not added during construction to block one of the entranceways so that bobcats could walk entirely through

the device (Figure 2). Thirdly, gun brushes used for sampling, located in the entranceways, were staggered to allow the collection of samples from bobcats that either entered the device entirely, or placed only their heads in the device. For the remainder of the manuscript this device will be 
referred to as hair snare cubby, or more simply as cubby. Specific considerations that contributed to the final design of the device were the appeal of the cubby to bobcats, the ability to collect a hair sample without behavioral rubbing, the ease of deploying the cubby, and the ability to set up the cubby without requiring specific vegetation or terrain features. Many of the final design considerations were made after extensive consultation with a professional bobcat trapper (personal communication: Mercer Lawing, Camtrip Cages). Each cubby was equipped with four 0.30 caliber bronze gun brushes bent inwardly that were used to collect hair samples from bobcats.

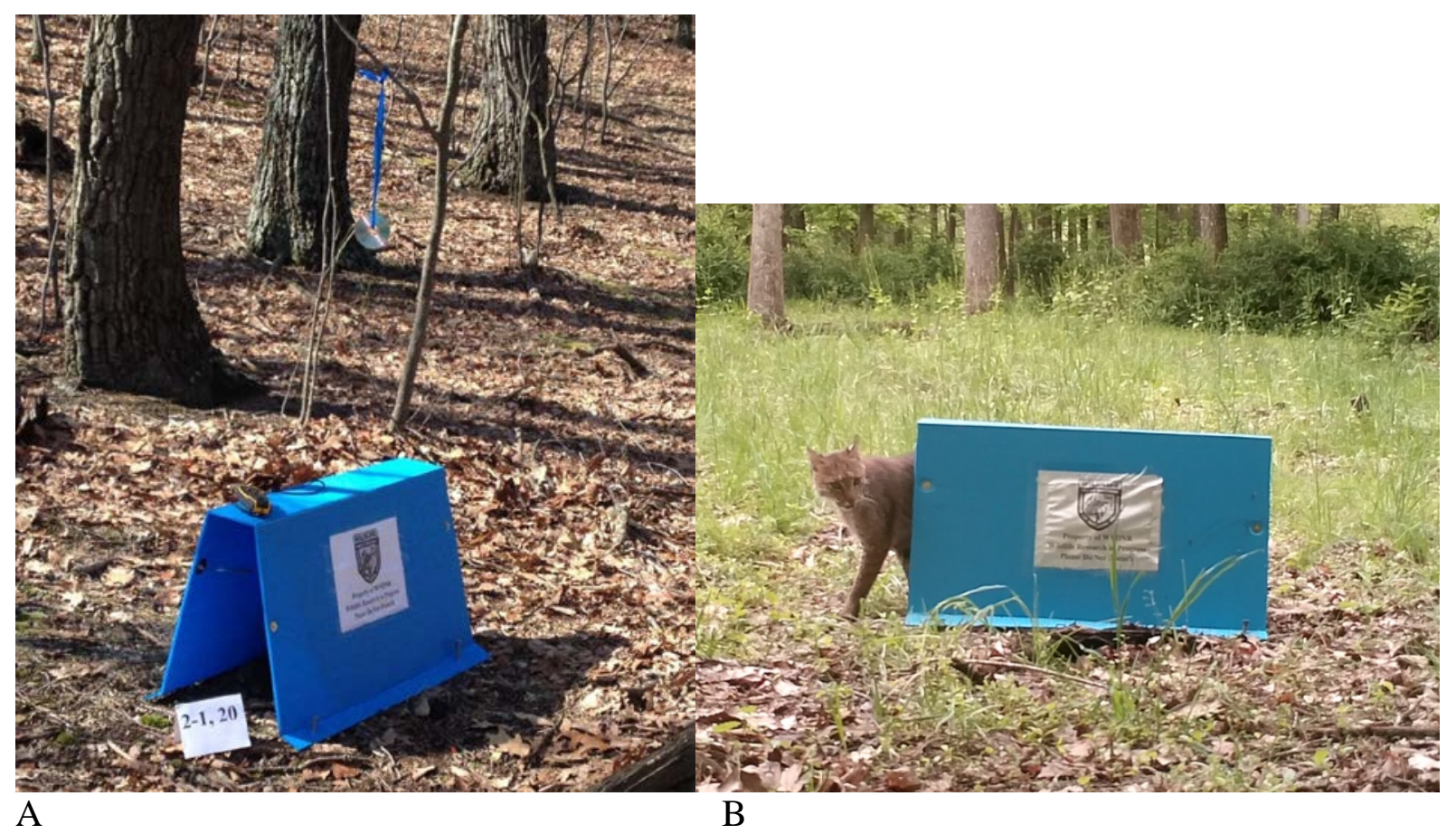

Figure 2 (A \& B) - (A) Bobcat hair snare cubby deployed in West Virginia. (B) Bobcat being sampled as it walks through the hair snare cubby.

Each hair snare cubby was constructed from a $111.7 \mathrm{~cm}$ x $81.3 \mathrm{~cm}$ pad of blue corrugated plastic pad of 9.5mm thickness (Figure 3). These plastic pads were then bent into a trapezoidal shape on the corner of a lab bench such that the flat apex of the cubby would stand roughly $44 \mathrm{~cm}$ from the ground—high enough for the average bobcat to enter without crouching. Each cubby 
was affixed with four $5.1 \mathrm{~cm}$ long \#8-32 bolts which were placed, two at each entrance, $2.6 \mathrm{~cm}$ from the outside edge and either $14.0 \mathrm{~cm}$ or $21.6 \mathrm{~cm}$ from the nearest apex crease. The bolts were staggered at each opening so that the equipped gun brushes would be at different heights to collect samples from animals even if varying entering postures were used. These bolts were then secured tightly using two sets of fender washers to provide support over as large of a surface area as possible and nylon-centered locking nuts. Once attached, the bolts were bent inward toward the center of the cubby and parallel to the ground so that the tip of the bolt was roughly $2.6 \mathrm{~cm}$ from the side wall of the cubby. Coupling nuts of \#8-32 thread and length $1.6 \mathrm{~cm}$ were then threaded onto the bolts and served as the attachment point for the gun brushes used in sampling. A $20.3 \mathrm{~cm}$ x $20.3 \mathrm{~cm}$ square piece of brown outdoor carpet was attached to the upper middle of the inside surface of each cubby using roughly 10 staples of approximately $9.5 \mathrm{~mm}$ in length. Supplies required for the construction and equipping of one cubby were roughly \$25 USD. 


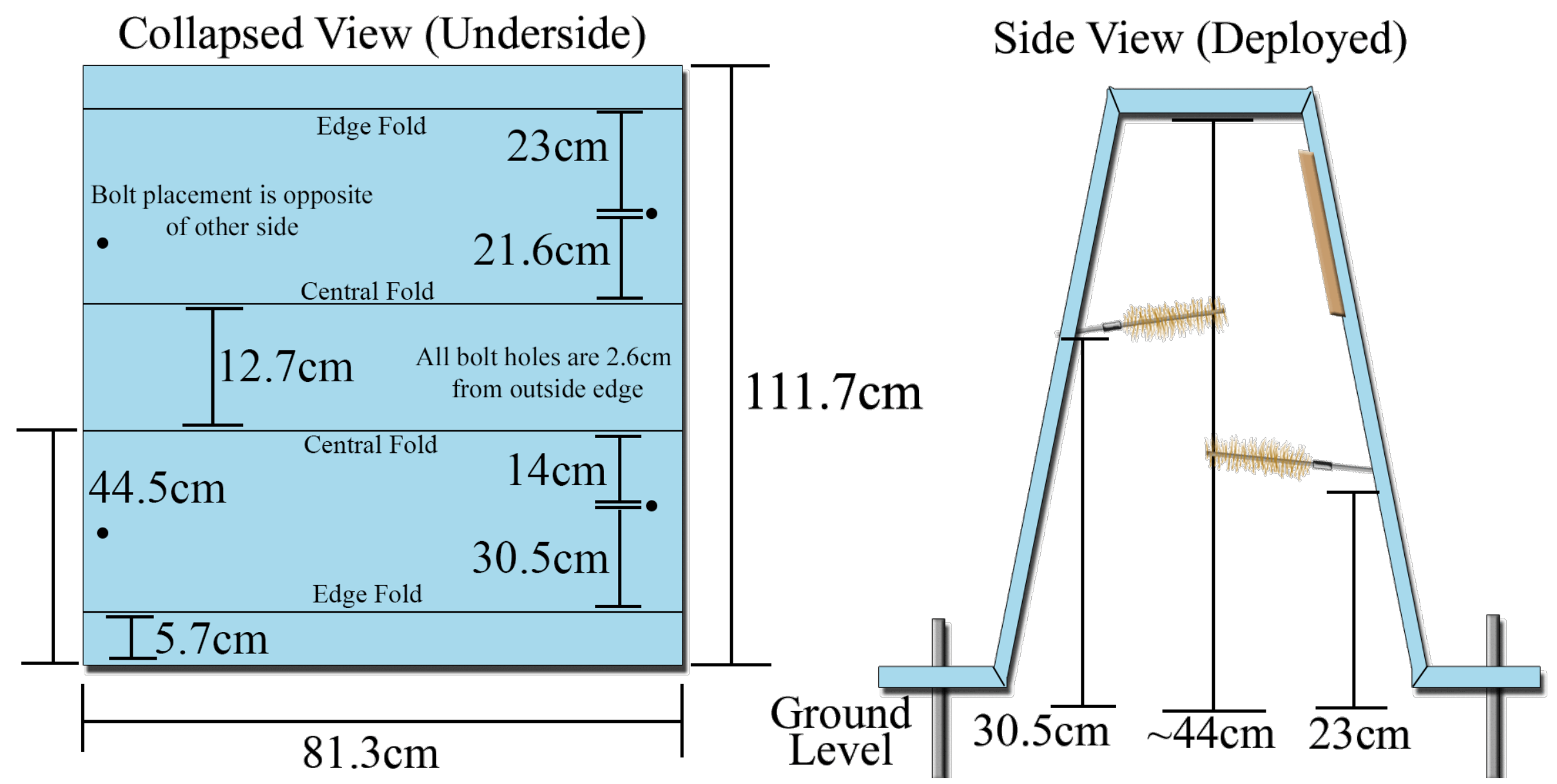

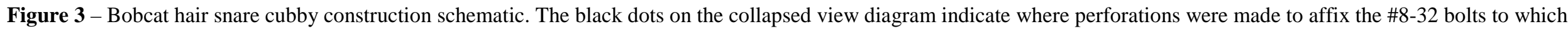

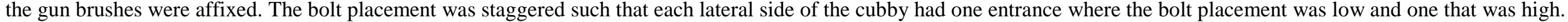

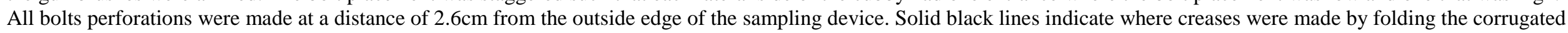

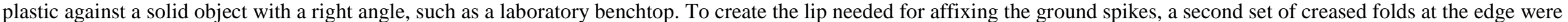

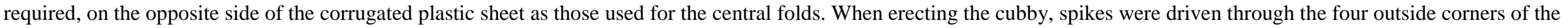
device at locations roughly $2.6 \mathrm{~cm}$ from any outside edges. 


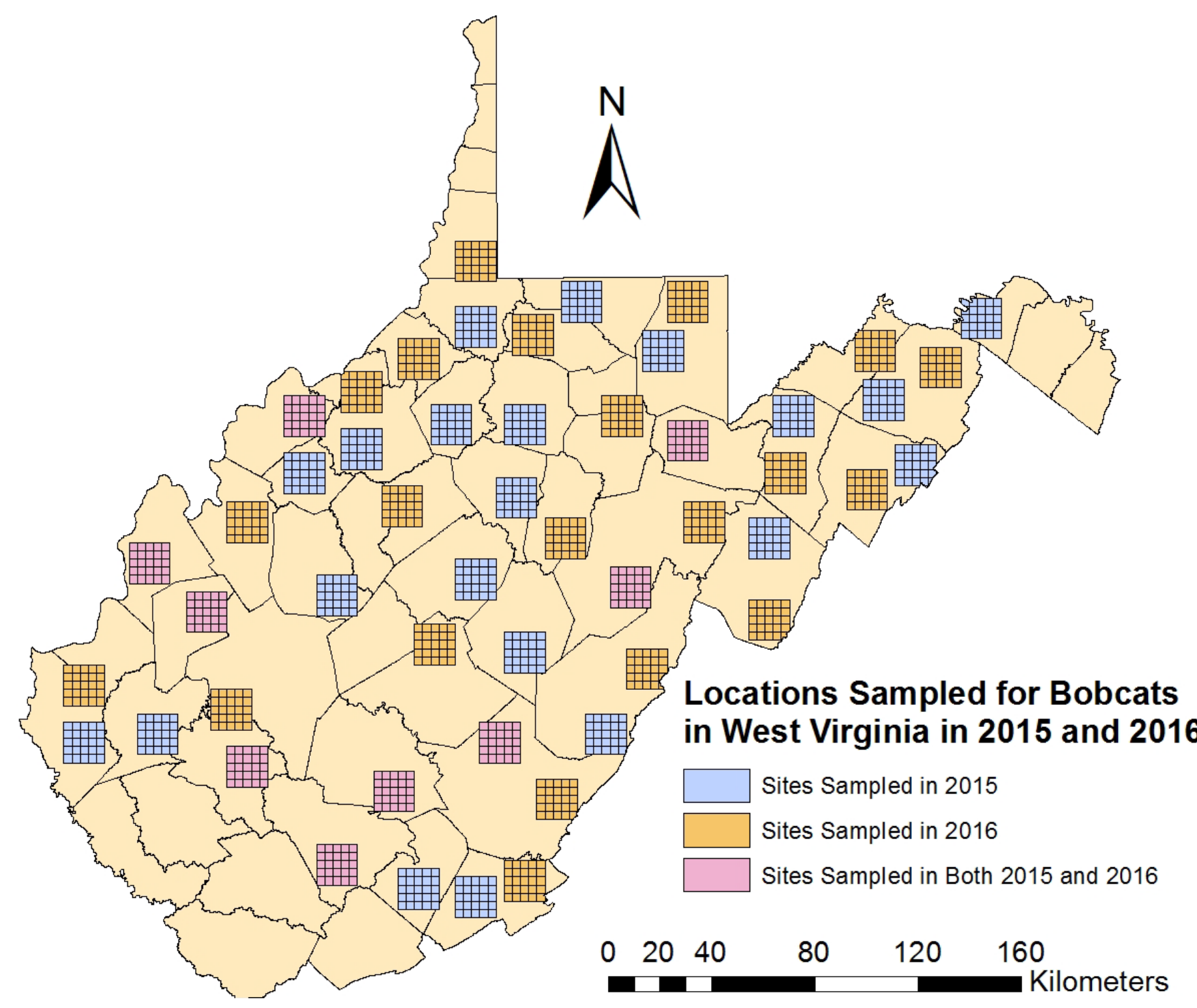

Figure 4 - Map of the sampled grid locations in West Virginia during the 2015 and 2016 field sampling seasons. Locations in blue were sampled only in 2015, orange sites were only sampled in 2016, and pink sites were sampled both in 2015 and 2016. A single hair snare cubby was placed in each of the cells of the 5x5 grid during sampling. Sampling took place at each location over the course of a four-week session. For both 2015 and 2016 a total of five sampling sessions were undertaken (Table 1). A total of six 5x5 grids were sampled simultaneously during the same session, with a total of 30 grids (7,500km2) being sampled in 2015 another 30 in 2016. 


\section{Field Methods}

Hair snare cubbies were deployed across the state of West Virginia as part of a study to assess the population model currently used to help manage West Virginia’s bobcat population. During the 2015 and 2016 field seasons, 30 individual 250 $\mathrm{km}^{2}$ study areas were sampled, for a total sampled area of 10,500 $\mathrm{km}^{2}$ across both years (Figure 4). Each of these locations were composed of a $5 \times 5$ grid of 25 equally sized $\left(10 \mathrm{~km}^{2}\right)$ cells to ensure even sampling efforts across each study area. Within each cell, a single hair snare cubby was placed over a 4-week session, for a total of 700 trap nights at a single site. Sampling took place simultaneously in six different grids with a total of five sessions taking place during March - August of 2015 and March - July of 2016 (Table 1). Cubby placement inside of each cell was opportunistic based on landowner approvals as well as available habitat and terrain types. When available, cubbies were selectively placed on or adjacent to game trails or dirt roads located within forested habitats with nearby edge, as recommended by Clare et al. (2015), for maximizing bobcat detection. However, many cubbies were deployed in suboptimal locations or habitats due to extraneous circumstances to provide useful habitat selection data for occupancy modeling and spatially-explicit capturerecapture (SECR).

Table 1 - Sampling dates for each session for both the 2015 and the 2016 sampling seasons.

\begin{tabular}{ccc}
\hline Session Number & 2015 & 2016 \\
\hline Session 1 & $3 / 23 / 15-4 / 20 / 15$ & $3 / 2 / 16-3 / 30 / 16$ \\
Session 2 & $4 / 21 / 15-5 / 19 / 15$ & $3 / 31 / 16-4 / 28 / 16$ \\
Session 3 & $5 / 20 / 15-6 / 17 / 15$ & $4 / 29 / 16-5 / 20 / 16$ \\
Session 4 & $6 / 18 / 15-7 / 16 / 15$ & $5 / 31 / 16-6 / 28 / 16$ \\
Session 5 & $7 / 17 / 15-8 / 14 / 15$ & $6 / 29 / 16-7 / 27 / 16$ \\
\hline
\end{tabular}


Once a site was selected for cubby deployment, a trowel was used to dig a $7.62 \mathrm{~cm}$ deep hole under what would become the center of the cubby. In this hole, $4 \mathrm{~g}$ of Caven's Minnesota Brand Bobcat Chunk Bait (Minnesota Trapline Products, Pennock, MN) was added as a food enticement for animals to enter the sampling device. Next, a handful of pillow stuffing was placed in the same hole and arranged as a visual attractant to look like the nest of a rodent. The cubby was then oriented over the mimic cache hole and one long side was secured to the ground using two $30.5 \mathrm{~cm}$ landscaping spikes driven directly through the edge flaps, roughly $2.5 \mathrm{~cm}$ from any outside edge. The cubby was then flipped and laid over the secured spikes such that the carpet was exposed.

Along the top half of the carpet square when the cubby was erected, $0.5 \mathrm{~g}$ of Light Skunk paste calling lure (Cage Magic Lures, Barstow, CA) was smeared. Along the bottom half of the carpet pad, $7 \mathrm{~mL}$ of a scent lure was added in an "S" pattern to cover as much surface area as possible. This lure was based on the recommendations of McDaniel et al. (2000) and was a mixture of 3,800mL of Beaver Castoreum Tincture (Kaatz Bros. Lures, Savanna, IL), 250mL glycerin, 250mL propylene glycol, and 250mL of Imitation Catnip Oil (Kaatz Bros. Lures, Savanna, IL). The glycerin and propylene glycol were added to the lure to prevent freezing. To each of the \#8-32 bolts inside of the cubby, a 0.30 caliber gun brush was attached using a $1.6 \mathrm{~cm}$ length threaded joining nut. The cubby was then erected such that the distance between the bottom of the two inside edges of each opening was $30.5 \mathrm{~cm}$, using a landscaping spike as a guide. Finally, two additional $30.5 \mathrm{~cm}$ landscaping spikes were driven through the unsecured side to firmly attach the cubby to the ground. As an additional visual attractant, a compact disc was hung from a nearby tree with long sight distance. The disc was tied to a roughly $61 \mathrm{~cm}$ length of 
colored flagging tape and oriented roughly $91.5 \mathrm{~cm}$ from the ground to ensure that wind would disturb the object and appeal to felid curiosity.

Deployed cubbies were checked every 7 days with all four gun brushes being collected and sealed in the same labeled manila envelope, regardless if hair was or was not visually apparent. During the weekly checks, scent baits and lures were also replaced along with the gun brushes. Following the second week of data collection, the cubbies were moved roughly 15m to alleviate a potential trap avoidance response due to familiarity (Kendall et al. 2008). Any unconsumed baits and visual attractants were removed from the old site and the previous cache hole was filled in with earth. At the conclusion of each four-week sampling period, all cubbies were removed from sampling locations and were reused to establish a sampling grid in a new location.

\section{Laboratory and Molecular Methods}

Gun brushes collected in the field were examined under a dissecting microscope for the presence of animal hair. If animal hair was observed, it was examined morphologically and the putative species of the sample was assigned using hair identification keys and resources of Hairdatabase.com (Knecht et al. 2015). Snared hairs were then individually removed from the gun brushes using a cleaned forceps. Hairs collected from brushes at the same site and week were combined and placed into a single $1.5 \mathrm{~mL}$ microcentrifuge tube containing $100-125 \mu \mathrm{L}$ of molecular grade distilled water. Both hair shafts and hair follicles were retained to ensure that as much DNA as possible was available for genetic analyses. The hair samples were then stored at $20^{\circ} \mathrm{C}$ until DNA extraction procedures. 
Used gun brushes were cleaned and reused throughout the project. The cleaning procedures consisted of an initial water wash with manual agitation in a plastic bin to remove any remaining coarse vegetative debris. The bin was filled and emptied of water a total of 5 times. Next, brushes were stacked on a grill grate and a laboratory burner was passed slowly underneath to burn off any missed hair fragments. The brushes were then flipped and the same process was repeated to the side previously unexposed to the flame. Once flame-treated, the brushes were washed again in the previously described manner another 5 times. To remove any remaining DNA that could contaminate new hair samples, entire gun brushes (except for the screw threads since they were easily damaged) were submerged in $3 \%$ hydrogen peroxide for a total of 10 minutes. The gun brushes were removed promptly to ensure that metal degradation did not occur. Following an additional 3 washing cycles, a laboratory burner was used to partially dry the brushes before they were stacked on aluminum foil to air dry. The cleaned brushes were then packaged and reused in field procedures.

DNA extraction was completed using a Qiagen (Bethesda, MD) DNeasy 96 Blood \& Tissue kit with a modified protocol to accommodate the hair samples. To each tube containing hair samples and water, $20 \mu \mathrm{L}$ of proteinase $\mathrm{K}(20 \mathrm{mg} / \mathrm{mL}), 30 \mu \mathrm{L}$ of $1 \mathrm{M}$ DTT (dithiothreitol), and $250 \mu \mathrm{L}$ of buffer ATL were added. The samples were then incubated at $56^{\circ} \mathrm{C}$ on a slowly rotating platform between 5 and 12 hours until the hairs were completely dissolved. The remainder of the extraction procedures followed the manufacturer's suggested protocol except that the final elution step was comprised of two $100 \mu \mathrm{L}$ buffer AE elution steps. Following elution, the 96-well extraction plates were dehydrated in a vacuum centrifuge at $60^{\circ} \mathrm{C}$ until roughly $75 \mu \mathrm{L}$ of buffer AE remained in each well of the plate (roughly 6 hours) to further concentrate the DNA. 
The polymerase chain reaction (PCR) was used to determine which of the collected hair samples originated from bobcats. Previous non-invasive felid studies identified species of origin by amplifying 300—1,000nt fragments of mitochondrial DNA (mtDNA) using universal primers followed by restriction enzyme digest or DNA sequencing (Foran et al. 1997, Mills et al. 2000, Weaver et al. 2005). Non-invasively collected samples often yield low quantity and low-quality DNA, and more recently, Stricker et al. (2012) targeted smaller DNA fragments since these are more likely to remain intact even after being exposed to the environment. Even with the use of smaller DNA amplicons, a high percentage of collected hair samples still fail in DNA sequencing when using traditional methods. As a part of this study, new quantitative PCR (qPCR) primers, specifically for bobcat research, were developed to reliably speciate samples of both high and marginal quality. qPCR also can be used to determine species of origin from mixed species samples, unlike the traditional methods that require DNA sequencing.

Two separate qPCR reactions using newly developed primer pairs were applied to determine species of origin from hair samples collected in this study. The first primer pair (FelidV-F: 5'-CCTATTTAACCTACCACACCCACAAG-3' and FelidV-R: 5'-GCCAGATGCTTTGTTTAAGCTACATC-3') was designed specifically to amplify a 105nt fragment of mtDNA from most wild felids native to or commonly found in North America including the bobcat, Canada lynx, cougar (Puma concolor), domestic cat (Felis catus), ocelot, and jaguarondi (Puma yagouaroundi). The second primer pair (LynX-F: 5’GCAAACATCAGCACCTCCGTT-3' and LynX-R: 5'CTAGTAGGGTGAAGACGTAGG CTTG-3') was developed to amplify a 109nt fragment of mtDNA from only two felid species native to North America — the bobcat and Canada lynx. The two-step species identification procedure began by using primer set FelidV to determine which hair samples were derived from 
a felid source. Next, primer set LynX was used on each sample testing positive for felid DNA to determine which samples originated from bobcats. Since the study area is not located within the known range of the Canada lynx, any sample which produced a PCR product for primer set LynX was considered to have been collected from a bobcat.

Preliminary testing of the technique using known tissue and hair samples revealed that both primer sets reliably amplified bobcat DNA extracts created from only two small ( $\sim 5 \mathrm{~mm}$ ) hairs without follicles. PCR reagents and reaction conditions for primer sets FelidV and LynX were identical: $5 \mu \mathrm{L}$ of (2x) Bio-Rad iQ SYBR Green Supermix (Hercules, CA), $1 \mu \mathrm{L}$ of nuclease-free distilled water, $0.5 \mu \mathrm{L}$ each of the appropriate two primers $(10 \mu \mathrm{M}$ concentration), and $3 \mu \mathrm{L}$ of DNA template- $\mathrm{a} 10 \mu \mathrm{L}$ total reaction size. Thermal cycling and PCR product quantification took place on a Bio-Rad CFX96 Touch Real-Time PCR Detection System under the following conditions: $95^{\circ} \mathrm{C}$ for 3 minutes followed by 40 cycles of $95^{\circ} \mathrm{C}$ for 10 seconds and $60^{\circ} \mathrm{C}$ for 30 seconds with a quantification image taken at the conclusion of each step. Samples were considered positive for primer set FelidV if the CQ value of the sample was $\geq 33.5$ and samples were considered positive for primer set LynX if the CQ value of the sample was $\geq 32.5$. These cutoff values were determined based on the CQ values produced by a positive control sample of two bobcat hairs, which was considered to be the lower threshold limit to the sensitivity of this analysis. On each plate of hair samples analyzed, at least three replicates of two bobcat hair positive controls were run to ensure the consistent accuracy of these cutoff thresholds. Due to budgetary constraints, only samples suspected by microscopy to be of felid origin (Hairdatabase.com 2015), those containing five or more hairs, or samples of less than five hairs with entire follicles were extracted and used for further analyses. 


\section{Results}

Of the total 2,014 hair samples collected in the 2015 sampling season, 1,641 yielded a sample suitable for genetic analysis (i.e., containing at least five or more hairs or a single hair with a follicle). In 2016, a total of 2,108 hair samples were collected, of which 1,301 were suitable for genetic analysis. Of the 6,000 individual site-week samplings that took place, $49 \%$ $(2,942 / 6,000)$ yielded a hair sample that was used in qPCR. qPCR using primer set FelidV revealed that in 2015, $21.7 \%(438 / 2,014)$ of the collected hair samples were derived from a felid source, as compared to the $16.0 \%(337 / 2,108)$ of 2016 . Subsequent analysis of these felidpositive samples with primer set LynX determined that 278 (63.5\%) and 100 (29.7\%) of these samples originated from bobcats, for the 2015 and 2016 sampling seasons, respectively. The remaining felid-positive samples that did not have a CQ $\geq 32.5$ for primer set LynX were assumed to have been collected from domestic cats. However, the actual number of positive individuals for LynX and felidV is possibly higher than recorded since no universal mtDNA control was used for each DNA extract to validate a positive qPCR reaction during testing procedures.

Since each site was analyzed for a total of 700 trap nights, and a total of 60 sites were examined, the performance of the bobcat hair snare cubby was evaluated for 21,000 trap nights each for both the 2015 and 2016 seasons. The 2015 season produced 278 confirmed bobcat detections, for a rate of 1.32 per 100 trap nights. The 2016 sampling season produced 100 confirmed bobcat detections, for a rate of 0.47 per 100 trap nights. When considering both sampling seasons together, a total of 378 bobcat detections were recorded for 42,000 trap nights, a rate of 0.9 detections per 100 trap nights (Table 2). 
Table 2 - Comparison of the detection rate performance of the bobcat hair snare cubby with other hair snares specifically designed to sample bobcats.

\begin{tabular}{|c|c|c|c|}
\hline Hair Snare Type & $\begin{array}{c}\text { Total Bobcat } \\
\text { Detections }\end{array}$ & $\begin{array}{c}\text { Trap } \\
\text { Nights }\end{array}$ & $\begin{array}{c}\text { Bobcat Detections / } 100 \\
\text { Trap Nights }\end{array}$ \\
\hline 2015 Season & 278 & 21,000 & 1.32 \\
\hline 2016 Season & 100 & 21,000 & 0.47 \\
\hline 2015-2016 Combined & 378 & 42,000 & 0.9 \\
\hline Cable Snares ${ }^{1}$ & 17 & 9,016 & 0.189 \\
\hline Scratch Pads ${ }^{2}$ & 0 & 2,072 & 0.000 \\
\hline Scratch Pads ${ }^{3}$ & 1 & 700 & 0.143 \\
\hline Scratch Pads ${ }^{4}$ & 1 & 1,680 & 0.060 \\
\hline
\end{tabular}

${ }^{1}$ Stricker et al. (2012) ${ }^{2}$ Long et al. (2007) ${ }^{3}$ Harrison (2006) ${ }^{4}$ Comer et al. (2011)

While a total of $26.3 \%(775 / 2,942)$ of all samples were collected from felid sources, additional DNA tests to determine species of origin were not conducted on the remaining nonfelid samples to reduce overall project costs. However, putative species of origin was assigned to these samples during hair removal procedures from the gun brushes. Anecdotally, the most common bycatch species were identified as follows, in order of descending occurrence: Virginia opossum (Didelphis virginiana), raccoon (Procyon lotor), black bear (Ursus americanus), and fisher (Martes pennanti). Despite the presence of coyotes (Canis latrans) at study sites, as recorded by camera traps, only a small number of samples were collected from this species. Anecdotal video evidence from these same camera traps revealed that when coyotes encountered a hair snare cubby, they frequently adopted a head-lowered posture of cautious curiosity, circled the device slowly, and then ran away. 


\section{Discussion}

The bobcat hair snare cubby is a newly developed tool that can be utilized by wildlife managers to collect hair samples from wild bobcats. When compared to other recent bobcat hair snare studies (Table 2), the bobcat hair snare cubby has between 2 - 6 times the detection rate per unit effort. However, bobcat density will impact detection per unit effort so these comparative results may not be correct in all situations. An example of this is the 2016 dataset from this study, where we sampled a number of locations thought to be poor bobcat habitat. These sites produced minimal numbers of bobcat detections and reduced the apparent effectiveness of the hair snare cubby, especially for the 2016 season. In contrast, the data collected from the 2015 field season was mainly from locations thought to be high quality bobcat habitat, which is reflected in the much higher number of detections per 100 trap nights.

While this device does require certain behaviors for an animal to be sampled, the curiosity to either completely enter the cubby, or at least investigate enough to come into contact with the gun brushes at the entryway, appears to be a fairly common behavior in bobcats, as compared to carpet scratch pads. However, advantages of using the bobcat hair snare cubby are not limited to increased detection rates when compared to other methods. Since hair samples are collected on gun brushes inside of the cubby, samples are not exposed to DNA-damaging moisture or UV light (Kendall et al. 2008) before sample retrieval, unlike the cable snares in Stricker et al. (2012), or the hair scratch pads first developed by McDaniel et al. (2000). The bobcat hair snare cubby is not a single-capture device, which is advantageous when collecting non-invasive samples from elusive carnivores. Single-capture devices only allow for the collection of genetic material from one individual before being inaccessible to the next, even if the initial capture is an undesired bycatch species. While samples consisting of DNA from 
multiple individuals of the same species can cause genotyping errors (Pauli et al. 2008), singlecapture devices run the risk of being triggered by more common species and then being unavailable to rarer ones, like bobcats. Since cubbies were deployed with four gun brushes at different heights at each of the two entrances, individual behavioral heterogeneity when interacting with the cubby was thought to reduce the possibility of sample cross-contamination.

The use of quantitative PCR is a highly sensitive approach to rapidly determine which collected hair samples originated from bobcats. The technique was found to be sensitive enough in this study to determine species of origin from samples as small as two small hairs without follicles. Following species identification, samples found to have originated from bobcats could then be genotyped for use in capture-recapture studies. Also, with a qPCR imaging device that can read more than one detector dye at a time, specific probes could be developed to identify multiple species of interest simultaneously in a single qPCR.

While the hair snare cubby was successful in collecting samples from wild bobcats, video observations made using camera-traps during field trials have resulted in suggestions that may improve capture success. Most bobcats were found to rub their cheek scent glands on the outside corner of the entryway of the cubby, regardless of whether they decided to enter the device. Affixing a hair snaring device to the entryways would result in greater capture success or greater DNA quantity than just using the four gun brushes already inside of the cubby. Also, as part of this project, many black bear bycatch samples were collected. When interacting with the cubbies, black bears often pulled the device from the ground or flattened it, rendering the cubby unable to collect bobcat samples until reset or replaced. It is recommended that use of cubbies in areas of high black bear density be scheduled for times of year when black bears are less active. 


\section{Management Implications}

The bobcat hair snare cubby is an effective device that can be deployed throughout the United States for use in non-invasive monitoring of bobcat populations or estimating abundance. Cubby deployment or checking can be completed in less than 10 minutes per site, regardless of the presence of trees or nearby terrain features. The newly described primers for use in qPCR provide a highly sensitive method to determine whether collected hair samples originated from bobcats.

\section{Acknowledgements}

We thank the many landowners of West Virginia for allowing us to sample their property for bobcats. We also thank the many members of the West Virginia Division of Natural Resources that worked on this project and made such a large-scale data collection effort possible_-especially Steve Rauch, Rob Silvester, Colin Carpenter, Eric Richmond, and Jeff McCrady. Finally, we thank Avery Owers, Brent Mays, Jarrod Ball, Brittany Demski, Lauren Schumacher, Brittney Watson, Peter Ries, Steven Wilson, Matt Stevens, Shelby Sherman, Kyle Matt, Sheila Harris, Darren Wood, and Paige Pritt from West Virginia University for their assistance in collecting hair samples from gun brushes and processing them in the laboratory. Partial financial support for this dissertation was provided by the West Virginia Division of Natural Resources through a grant with the U.S. Fish and Wildlife Service Wildlife and Sport Fish Restoration Program (W-48-R) and matching state funds. 


\section{References}

Beasom, S. L., and R. A. Moore. 1977. Bobcat Food Habit Response to a Change in Prey Abundance. The Southwest Naturalist 21:451-457.

Chamberlain, M. J., B. D. Leopold, L. W. Burger, B. W. Plowman, and L. M. Conner. 1999. Survival and Cause-Specific Mortality of Adult Bobcats in Central Mississippi. The Journal of Wildlife Management 63:613.

Chamberlain, M. J., B. D. Leopold, and L. M. Conner. 2003. Space Use, Movements and Habitat Selection of Adult Bobcats (Lynx rufus) in Central Mississippi. The American Midland Naturalist 149:395-405.

Clare, J. D. J., E. M. Anderson, and D. M. MacFarland. 2015. Predicting Bobcat Abundance at a Landscape Scale and Evaluating Occupancy as a Density Index in Central Wisconsin. The Journal of Wildlife Management 79:469-480.

Comer, C. E., M. E. Symmank, and J. C. Kroll. 2011. Bobcats Exhibit Low Detection Rates at Hair Collection Stations in East Texas. Wildlife Biology in Practice 7:116-122.

Crowley, S. M. and D. P. Hodder. 2017. An Assessment of the Efficacy of Rub Stations for Detection and Abundance Surveys of Canada Lynx (Lynx canadensis). Canadian Journal of Zoology 95:885-890.

DePue, J.E. and M. Ben-David. 2007. Hair Sampling Techniques for River Otters. Journal of Wildlife Management 71:671-674.

Foran, D. R., K. R. Crooks, and S. C. Minta. 1997. Species Identification from Scat: An Unambiguous Genetic Method. Wildlife Society Bulletin 25:835-839.

Harrison, R. L. 2006. A Comparison of Survey Methods for Detecting Bobcats. Wildlife Society Bulletin 34:548-552.

Heilbrun, R. D., N. J. Silvy, M. J. Peterson, and M. E. Tewes. 2006. Estimating Bobcat Abundance Using Automatically Triggered Cameras. Wildlife Society Bulletin 34:69-73.

Heilbrun, R. D., N. J. Silvy, M. E. Tewes, and M. J. Peterson. 2003. Using Automatically Triggered Cameras to Individually Identify Bobcats. Wildlife Society Bulletin 31:748755.

Kendall, K. C., K. S. McKelvey, and others. 2008. Hair collection. Noninvasive Survey Methods for Carnivores. Island Press, Washington, DC 141-182.

Knecht, L., T.F. Rounsville Jr., and J.E. Huffman. 2015. Dynamic Hair Identification Key Version 1.0B. Available at: http://www.hairdatabase.com. 
Larrucea, E. S., G. Serra, M. M. Jaeger, and R. H. Barrett. 2007. Censusing Bobcats Using Remote Cameras. Western North American Naturalist 67:538-548.

Long, R. A., T. M. Donovan, P. Mackay, W. J. Zielinski, and J. S. Buzas. 2007. Comparing Scat Detection Dogs, Cameras, and Hair Snares for Surveying Carnivores. The Journal of Wildlife Management 71:2018-2025.

McDaniel, G. W., K. S. McKelvey, J. R. Squires, and L. F. Ruggiero. 2000. Efficacy of Lures and Hair Snares to Detect Lynx. Wildlife Society Bulletin (1973-2006) 28:119-123.

Mills, L. S., K. L. Pilgrim, M. K. Schwartz, and K. McKelvey. 2000. Identifying Lynx and Other North American felids Based on MtDNA Analysis. Conservation Genetics 1:285-288.

Morin, D. J., L. P. Waits, D. C. McNitt, and M. J. Kelly. 2018. Efficient Single-survey Estimation of Carnivore Density Using Fecal DNA and Spatial Capture-Recapture: A Bobcat Case Study. Population Ecology 60:197-209.

Pauli, J. N., M. B. Hamilton, E. B. Crain, and S. W. Buskirk. 2008. A Single-sampling Hair Trap for Mesocarnivores. Journal of Wildlife Management 72:1650-1652.

Roberts, N. M., and S. M. Crimmins. 2010. Bobcat Population Status and Management in North America: Evidence of Large-Scale Population Increase. Journal of Fish and Wildlife Management 1:169-174.

Ruell, E. W., S. P. D. Riley, M. R. Douglas, J. P. Pollinger, and K. R. Crooks. 2009. Estimating Bobcat Population Sizes and Densities in a Fragmented Urban Landscape Using Noninvasive Capture-Recapture Sampling. Journal of Mammalogy 90:129-135.

Stricker, H. K., J. L. Belant, D. E. Beyer, J. Kanefsky, K. T. Scribner, D. R. Etter, and J. Fierke. 2012. Use of Modified Snares to Estimate Bobcat Abundance. Wildlife Society Bulletin 36:257-263.

Symmank, M., C. Comer, and J. Kroll. 2008. Estimating Bobcat Abundance in East Texas Using Infrared-triggered Cameras. Proceedings of the Annual Conference of the Southeastern Association of Fish and Wildlife Agencies 62:64-69.

Thornton, D. H., and C. E. Pekins. 2015. Spatially Explicit Capture-recapture Analysis of Bobcat (Lynx rufus) Density: Implications for Mesocarnivore Monitoring. Wildlife Research 42:394-404.

U.S. Fish and Wildlife Service. 1982. Proposal to Remove the Bobcat from Appendix II of the Convention on International Trade in Endangered Species of Wild Fauna and Flora. Federal Register 47:1242-1246.

Weaver, J. L., P. Wood, D. Paetkau, and L. L. Laack. 2005. Use of Scented Hair Snares to Detect Ocelots. Wildlife Society Bulletin 33:1384-1391. 


\title{
Chapter 3 - Modeling Bobcat Occupancy in West Virginia at the Landscape
}

\section{Scale}

\begin{abstract}
Occupancy models constructed using presence-absence data can be useful in determining the distribution of a species as well as the environmental and habitat variables that impact site selection at the landscape scale. Bobcats (Lynx rufus) are medium sized felids that are native to North America and are of conservation concern to wildlife management agencies due to their inclusion under CITES Appendix II. Since the bobcat is a wide-ranging and elusive species it can be difficult to collect distribution information, especially at the landscape spatial scale. This study used a newly developed bobcat hair snare cubby to collect hair samples from wild bobcats over the course of two field seasons. In 2015 and 2016 a total of 278 and 100 bobcat detections were recorded, respectively. Two occupancy models were constructed from this dataset, one from the 2015 data alone, and a second from the 2015-16 dataset combined. Average predicted probability of detection $(p)$ was $0.133 \pm 0.027$ and $0.098 \pm 0.014$, for the 2015 and the 2015-16 datasets, respectively. The average predicted occupancy probability $(\psi)$ was $0.858 \pm 0.057$ and $0.834 \pm 0.055$, for the 2015 and 2015-16 datasets, respectively. Bobcats were nearly ubiquitously distributed throughout West Virginia, and of the tested hypotheses, human influences on the landscape had the greatest negative impact on both the $p$ and $\psi$ of bobcats. However, bobcats were also found to be positively correlated with minor levels of disturbance and human activity, suggesting that bobcats may selectively occupy habitats with some disturbance due to the benefits of additional prey density and diversity afforded by small-scale environmental manipulations.
\end{abstract}




\section{Introduction}

Knowledge of the distribution of a species on the landscape is crucial to making informed management decisions. However, the collection of this information from rare or elusive carnivore species, like bobcats (Lynx rufus), can be a difficult undertaking. Even when sampling conditions are ideal, a survey can still fail to detect bobcats that are present in a particular area. Due to this lack of perfect detection, the data from sites that have bobcats that fail to be detected and sites that do not have bobcats can become confounded. To account for these issues with imperfect detection, a modeling framework was originally introduced by MacKenzie et al. (2002), and was coined as an “occupancy model.” The original occupancy model used a likelihood-based method to estimate species occupancy in sampled and unsampled locations by correlating the detection probability $(p)$ and occupancy probability $(\psi)$ with covariate descriptors, such as habitat type. While occupancy models have evolved, the occupancy model principles remain unchanged.

The most important steps in creating occupancy models useful to wildlife management lie in the strategies used to collect the presence and absence dataset for the model. Mackenzie and Royle (2005) published a guide on the proper techniques to use when collecting data to be used in occupancy modeling. According to these authors, site selection and sampling strategy are the two most important factors to consider. If only locations with perceived excellent habitat and high densities of the desired species are selected to be sampled, very little useful data will be collected regarding particular habitat covariates that may influence occupancy at specific sites.

The sampling strategy is highly dependent on the focal species of the research. For those species that have a high probability of being detected when a site is surveyed, the number of optimal surveys at the same site needed to gather accurate data is much less than for those 
species that have a low probability of detection. For species that have a high level of probability of occupancy ( $\psi$ ), only a few surveys are adequate, whereas those with a low level of $\psi$ require additional surveys. For rare and elusive carnivores that have a wide distribution but low density, like bobcats, Mackenzie and Royle (2005) recommended sampling more sites less intensively, rather than repeated surveys at the same locations. However, a minimum of 3 surveys at the same location was recommended to reduce the error associated with any single sampling event.

Since their introduction, occupancy models have been constructed for bobcats in a variety of locations and ecotypes. In Vermont, bobcat occupancy was best predicted by the percentage of mixed forest and forested wetlands located within $1 \mathrm{~km}$ of the site locations (Long et al. 2011). In Wisconsin, a camera trapping study found occupancy was most closely associated with percentage of woody cover and wetland edge near the camera site and that detection was positively correlated with nearby dirt road travel corridors (Clare et al. 2015). A camera-trapping study of the Appalachian Trail corridor found that bobcat occupancy was positively correlated with percentage of forest and distance to nearest road and negatively correlated with human activity (Erb et al. 2012). Wang et al. (2015) found bobcat occupancy and detection to be negatively associated with human landscape impact and activity at camera sites in California, and the same conclusion was reached by Lewis et al. (2015) in Colorado and Lesmeister et al. (2015) in southern Illinois. In an urban landscape of east Texas, Lombardi et al. (2017) reported that bobcat occupancy was best predicted by the percentage of forested habitat and the distance to the nearest building.

The majority of previous bobcat occupancy modeling studies, as described above, have determined that bobcat occupancy and detection probability ( $\psi$ and $p$ ) are most strongly influenced by the amount of forest cover and human impact on a landscape. While the amount of 
human impact on the landscape is also predicted to be of importance to $\psi$ and $p$ in West Virginia, forest cover is not expected to be as much of an important predictor. Unlike many of the previously discussed study locations, West Virginia is one of the most densely forested locations in the United States, with roughly $78 \%$ of the state's total area being comprised of mostly mature forests (Randall et al. 2016). Previous bobcat resource use research has documented that bobcats appear to select home ranges on the local scale based on abundance of prey, and not any particular habitat type (McCord 1974, Litvaitis et al. 1986, Chamberlain et al. 1999, 2003, Lesmeister et al. 2015). Within larger core forested tracts, bobcats are known to select early successional subsets as their primary locations of activity, presumably due to the greater prey density in these areas, as opposed to mature forests (Litvaitis et al. 1986, Chamberlain et al. 2003). Since bobcats selectively choose their home ranges based on prey availability, habitat complexity (not percentage of mature forests) is predicted to be the dominant determinant of bobcat $\psi$ in West Virginia, with human impacts being an important predictor for both $p$ and $\psi$.

Since the bobcat population of West Virginia has only relatively recently recovered from historical lows of the 1960s and 1970s (Roberts and Crimmins, 2010), it is likely that there are areas within West Virginia and the bordering states that currently do not have breeding bobcat populations. While habitat may be available, these areas are probably still being colonized by juveniles and yearlings from stable population sources (Kitchings and Story, 1984, Croteau et al., 2010). Detection-nondetection data can be valuable in determining the distribution of elusive carnivore species, as well as particular landscape variables influence bobcat occupancy in specific locations on a continuous landscape. Bobcats are assumed to range across the entirety of West Virginia, but the exact extent is unknown. Detection-nondetection data will be used to evaluate occupancy of bobcats at the landscape scale in West Virginia. The objectives of this 
research are to 1) construct a statewide occupancy model for the bobcats in West Virginia and 2) determine which environmental, landscape, and sampling variables impact bobcat detection probability or occupancy.

\section{Materials and Methods}

\section{Field Methods}

The dataset used for this study was collected as part of a WVDNR project to validate the population model currently used for management decisions. To collect presence and absence information from wild bobcat individuals throughout West Virginia, non-invasive hair snares were deployed statewide. The particular hair snare used, the hair snare cubby (see Chapter 2), was developed specifically for the WVDNR Bobcat Project (see Chapter 2). The hair snare cubby is constructed of thin, blue corrugated plastic and is erected like a tent such that an adult bobcat is able to move directly through the device. The hair snare cubby uses four 0.30 -caliber gun brushes, with two fixed to each entranceway, to collect hair from animals that enter from either side. A carpet pad affixed to the inside of the device was coated with two scent lures: a mixture of beaver castoreum and artificial catnip oil and a Light Skunk paste calling lure (Cage Magic Lures, Barstow, CA). In addition to the scent lures, a food bait of Caven’s Minnesota Brand Bobcat Chunk Bait (Minnesota Trapline Products, Pennock, MN) was placed in a mock cache hole dug directly under the center of the hair snare cubby.

To determine the locations to be sampled statewide, West Virginia $\left(\sim 63,000 \mathrm{~km}^{2}\right)$ was first divided into a latticework of cells $10 \mathrm{~km}^{2}$ in size. This cell size was selected according to recommendations of White (1982) for capture-recapture surveys, that at least two sampling devices should be placed into the smallest possible home range for the species of interest. While 
no direct home range data were available in West Virginia, average home range size of a female bobcat was estimated to be roughly $20 \mathrm{~km}^{2}$ in similar habitat in nearby Virginia (Progulske 1952, McCord and Cordoza 1982). From this latticework, a total of 30 individual $250 \mathrm{~km}^{2}$ clumped sampling sites were selected for intensive study, for both the 2015 and 2016 field seasons, for a total of 60 sites. Each $250 \mathrm{~km}^{2}$ study site was comprised of a $5 \mathrm{x} 5$ grid of 25 total $10 \mathrm{~km}^{2}$ cells, to ensure even sampling at each selected site. Clumped sampling locations were selected based on a number of criteria, the most important being an attempt to evenly spread out sampled locations and minimize the distance between them to maximize the accuracy of the final extrapolation. Other important criteria considered were ensuring that a diversity of habitat types and locations with both high and low suspected bobcat densities were sampled.

Within each $10 \mathrm{~km}^{2}$ cell selected for study, a single hair snare cubby was placed opportunistically based on available habitat types and landowner permissions if not placed on public land. When available, cubbies were selectively placed on or adjacent to game trails or dirt roads in forested habitats, as recommended by Clare et al. (2015) for increasing $p$. Due to extraneous circumstances such as issues in gaining landowner permissions, some cubbies were deployed in what was thought to be less than ideal bobcat habitat. Cubbies were left on site for a total of four 1-week periods, which will be referred to henceforth as a session. At the end of each week the four gun brushes from a single cubby were collected and placed in a manila envelope, whether or not a hair sample was visually apparent. Baits and scent lures were replenished along with the gun brushes each week of sampling. Also, between the second and the third weeks of the study the hair snare cubbies were moved $\sim 15 \mathrm{~m}$ in an attempt to alleviate a potential trap avoidance response (Kendall et al. 2008). Over the course of the 2015 and 2016 field seasons a total of five sampling sessions, of four weeks each were completed. These took place between 
March - August in 2015 and March - July in 2016, with six different 25-cell grids being deployed statewide at any given time (Table 1). The 2016 sampling season was shifted earlier in the year to minimize potential bear damage to cubbies during sampling. At the conclusion of a session, each cubby was removed and placed in a new sampling location.

\section{Laboratory and Molecular Methods}

The gun brushes collected and sealed in the field were examined for the presence of hair under a dissection microscope. Entire hairs were removed from each of the four gun brushes from a single cubby and placed into a $1.5 \mathrm{~mL}$ microcentrifuge tube containing $100-125 \mu \mathrm{L}$ of molecular grade distilled water. Hair samples were stored at $-20^{\circ} \mathrm{C}$ until DNA extraction was completed with a Qiagen (Bethesda, MD) DNeasy 96 Blood \& Tissue kit with a modified protocol. Into each tube containing hair samples and water, $20 \mu \mathrm{L}$ of proteinase $\mathrm{K}(20 \mathrm{mg} / \mathrm{mL})$, $30 \mu \mathrm{L}$ of $1 \mathrm{M}$ DTT (dithiothreitol), and $250 \mu \mathrm{L}$ of buffer ATL were added. Samples were then incubated at $56^{\circ} \mathrm{C}$ for $5-10$ hours until the entire hairs were completely dissolved. The remaining extraction procedures followed the manufacturer's suggested protocol except that the final elution step was comprised of two $100 \mu \mathrm{L}$ buffer AE elution steps. The second elution was used to ensure that as much DNA as possible was collected from the filter. Once eluted, sample plates were dried on a vacuum centrifuge at $60^{\circ} \mathrm{C}$ until about $75 \mu \mathrm{L}$ of buffer $\mathrm{AE}$ remained in each well of the plate (roughly 6 hours) to further concentrate the DNA. Due to budgetary constraints, only samples suspected by microscopy to be of felid origin (Hairdatabase.com 2015), those containing five or more hairs, or samples of less than five hairs with entire follicles were extracted and used for further analyses. 
Species identification of collected hair samples was completed using the polymerase chain reaction (PCR). Two new primer pairs were specifically developed for this study to allow for reliable qPCR species identification from degraded or fragmented hair samples that might otherwise fail to be Sanger sequenced, yet still have enough DNA for qPCR speciation (see Chapter 2). The first primer pair, FelidV was used to determine which samples were of felid origin, and the second primer pair LynX, only amplifies the mitochondrial DNA (mtDNA) of the bobcat and Canada lynx (Lynx canadensis). To identify species, a two-step PCR procedure was used: an initial qPCR with FelidV identified which samples were of felid origin and then a separate qPCR with primer set LynX was used to determine which felid samples originated from a bobcat. Positive and negative controls were run with each qPCR reaction, and were used to establish a CQ cutoff for a positive result. After multiple runs, it was determined that a control sample of two bobcat hairs (the minimum considered to be needed to produce a usable sample), which became the positive cutoff, would reliably have a CQ of roughly 33.5 for the FelidV primer set and a CQ of roughly 32.5 for the LynX primer set. As such, samples producing a CQ value greater than these critical thresholds were considered positive for that particular reaction. Samples producing a positive result for the $\mathrm{V}$ primer set, but a negative result for $\mathrm{X}$ were assumed to have originated from domestic cats (Felis catus), since this is the only other felid species known to inhabit the sampling areas. Canada lynx are not known to inhabit West Virginia, so a positive result for qPCR using primer set LynX was recorded as a bobcat detection.

\section{Occupancy Modeling}

Landscape and sampling covariate data were collected to explain hypotheses generated that best explain the detection probabilities ( $p$ ) and occupancy $(\psi)$ of bobcats across West 
Virginia. These hypotheses are explained in detail in the following section. The habitat covariates used in this study for the occupancy modeling procedures originated from a number of sources (Table 3). The road dataset used in this study was derived from the 2015 TIGER line roads dataset, as available from the United States Census Bureau (2017). The primary (S1100), secondary (S1200), and minor (S1300) road categories were left unmodified and were used in this study for each of these respective classes. A new road category, DST_TRL, or the distance to trail was created by combining the roads classified as $4 \times 4$ trail (S1500), walkway / pedestrian (S1710), private roadway (driveways, logging roads, gas well roads) (S1740), and bike path or trail (S1820) into a single dataset. The distance to each of these types of roads was calculated using the Distance to Feature Tool of ArcGIS v.10.4 (ESRI, Redlands, CA). The overall road density for West Virginia $\left(\mathrm{km} / \mathrm{km}^{2}\right)$ (ROAD_DEN) was calculated using a combined dataset of the above road types and the Line Density tool from the ArcGIS v.10.4 Spatial Analyst package. A pixel-meter raster cell size was used ( $30 \mathrm{x} 30)$ and from each of these pixels the road density within $2,500 \mathrm{~m}$ was calculated. The road density value used for the analysis was taken from the pixel that intersected the hair snare cubby's physical location.

The West Virginia Land-Use Land Cover dataset (LULC) (Maxwell et al. 2012), which is a $9 \mathrm{~m}^{2}$ raster of major landcover classes, was used to create a new raster of all open water (lakes, streams, and certain areas of wetlands) for use in calculating the distance from each hair snare cubby location to the nearest source of water (DST_WA) with first the Raster to Points tool, and then the Distance to Points Tool of ArcGIS v.10.4. The elevation data of each cubby location (ELEV) was collected using the Raster to Points Tool of ArcGIS v.10.4 and the 2016 elevation DEMs constructed by the United States Geological Survey (USGS) for West Virginia. 
The landscape percentage covariates, Simpson’s Diversity Index (SIDI), deciduous forest largest patch index (LPI_DFOR), and edge density (ED) were all calculated using FRAGSTATS v4 (McGarigal et al. 2012). The source data for these analyses was the 2011 National Land Cover Dataset produced by USGS (Homer et al. 2015). To simplify the number of covariates included in the analysis, a number of categories of the NLCD were combined. Firstly, categories 21 (development open) and 22 (development low intensity) were combined to create the LOWDEV class. Next, categories 23 (development medium) and 24 (development high) were combined to create the HIDEV class. Categories 81 (pasture / hay) and 82 (cultivated crops) were combined into the AG class, and categories 90 (woody wetlands) and 95 (emergent herbaceous wetlands) were combined to the new WETL class. However, due to the low overall percentages of wetland landcover in West Virginia, this covariate was not considered in further analysis.

The FRAGSTATS analysis was completed at a fixed radius of either $1 \mathrm{~km}$ or $2.5 \mathrm{~km}$ around each cubby hair snare site. These buffer sizes were selected since they were roughly either $20 \%$ or $100 \%$ of the suspected home range size of an adult female bobcat in West Virginia, as was used by Lesmeister et al. (2015) in southern Illinois. The average home range size of bobcats in West Virginia is estimated to be roughly $20 \mathrm{~km}^{2}$, based on the work of Progulske (1952) in similar habitats of Virginia. A $1 \mathrm{~km}$ buffer radius produces a total sampled area of roughly $3 \mathrm{~km}^{2}$ and a $2.5 \mathrm{~km}$ buffer produces a total sampled area of nearly $20 \mathrm{~km}^{2}$. A $20 \%$ and $100 \%$ buffer percentage were calculated for each of the habitat type variable used in the analysis. However, Pearson's correlation analyses discovered that each of the pairs of buffer sizes were highly correlated ( $\mathrm{R}>0.6$ ), so only the $2.5 \mathrm{~km}$ buffer sizes were used in this study, except for the percentage of deciduous forest. However, both the $1 \mathrm{~km}$ and $2.5 \mathrm{~km}$ values were not 
used in the same model. Since the goal of this chapter is to create a statewide occupancy model, a larger buffer size $(2.5 \mathrm{~km})$ is more appropriate to discover the macro-scale relationship of bobcats to the landscape rather than fine-scale habitat selection.

Additional covariate information was provided by the WVDNR in the form of ecoregional mast data collected using the methods of Uhlig and Wilson (1952) for 2010 - 2016. These mast values were used as a proxy for estimated differences in prey abundance across the ecological regions of West Virginia.

A final correlation analysis of all covariates collected for use in the study was completed to remove any potential sources of multicollinearity. For any covariate pairs found to be significantly correlated $(\mathrm{R}>0.6)$, one of the covariates explaining the same phenomena was removed from the study. PER_LOWDEV was removed from the study in this manner since it was found to be highly correlated with ROAD_DEN, but ROAD_DEN was retained because it was thought to hold more important information for modeling. 
Table 3 - Covariates used to model probability of detection $(p)$ and occupancy probability $(\psi)$ in bobcats of West Virginia. The predictor type is the parameter estimates for which that covariate was used. The expected result is the direction of the correlative relationship between each covariate and bobcat $p, \psi$, or both. Hypotheses associated with conclusions reached from other studies are marked with a superscript.

\begin{tabular}{|c|c|c|c|}
\hline Covariate & Description & $\begin{array}{l}\text { Predictor } \\
\text { Type }\end{array}$ & $\begin{array}{l}\text { Expected } \\
\text { Result }\end{array}$ \\
\hline BearDAM & $\begin{array}{l}\text { A categorical variable for whether or not hair snare cubby at a site was damaged (1) or not (0) by } \\
\text { an encounter with an American black bear (Ursus americanus) during an entire session. }\end{array}$ & $p$ & - \\
\hline DST_MR & Linear distance (m) to nearest minor paved road. & $p, \psi$ & $+1,2$ \\
\hline DST_PR & $\begin{array}{l}\text { Linear distance }(\mathrm{m}) \text { to nearest interstate highway. These roads are travelled by large volumes of } \\
\text { traffic in certain areas but also pass through otherwise remote locations. }\end{array}$ & $p, \psi$ & $+1,2$ \\
\hline DST_SR & Linear distance (m) to nearest secondary road, such as state routes. & $p, \psi$ & $+1,2$ \\
\hline DST_TRL & Linear distance (m) to nearest foot path, off-road vehicle trail, or dirt road. & $p$ & -1 \\
\hline DST_UC & $\begin{array}{l}\text { Linear distance (m) to nearest urban cluster, or locations with populations }>2,500 \text {, as defined by } \\
\text { the U.S. Census Bureau for } 2015 \text {. }\end{array}$ & $p, \psi$ & $+^{2}$ \\
\hline DST_WA & Linear distance (m) to nearest source of open water, whether streams, lakes, or wetlands. & $\psi$ & -1 \\
\hline ED & Measurement of edge density calculated within a 2,500m radius from hair snare cubby location. & $\psi$ & + \\
\hline ELEV & Elevation (m) of hair snare cubby location. & $\psi$ & + \\
\hline LPI_DFOR & $\begin{array}{l}\text { Largest patch index of deciduous forest located within a radius of 2,500m of hair snare cubby } \\
\text { location. }\end{array}$ & $\psi$ & + \\
\hline MAST & $\begin{array}{l}\text { Calculated mast index using the method of Uhlig and Wilson (1952) by West Virginia Division of } \\
\text { Natural Resources for each of the state's six ecological regions between 2010-2016. Locations } \\
\text { with higher mast indices are thought to be correlated to greater prey density. }\end{array}$ & $p, \psi$ & + \\
\hline PER_AF & $\begin{array}{l}\text { Percentage of landscape that is covered with any type of forest within a radius of } 2,500 \mathrm{~m} \text { of the } \\
\text { cubby hair snare location. }\end{array}$ & $\psi$ & $+1,2,3$ \\
\hline PER_AG & Buffer $(2,500 \mathrm{~m})$ calculated percentage of land cleared for and being used to grow crops. & $\psi$ & - \\
\hline $\begin{array}{l}\text { PER_DFOR } \\
-1000\end{array}$ & $\begin{array}{l}\text { Percentage of the landscape that is deciduous forest within a radius of } 1,000 \mathrm{~m} \text { from hair snare } \\
\text { cubby location. This covariate was found to be highly correlated }(\mathrm{R}>0.6) \text { with } \\
\text { PER_DFOR_2500. However, it was included in further analyses, but only those that did not } \\
\text { contain both variables simultaneously. }\end{array}$ & $p$ & + \\
\hline $\begin{array}{l}\text { PER_DFOR } \\
2500\end{array}$ & $\begin{array}{l}\text { Percentage of the landscape that is deciduous forest within a radius of } 2,500 \mathrm{~m} \text { from hair snare } \\
\text { cubby location. }\end{array}$ & $\psi$ & + \\
\hline PER_EFOR & Percentage of evergreen forest within a radius of 2,500m of hair snare cubby location. & $\psi$ & $0^{5}$ \\
\hline PER_GRASS & $\begin{array}{l}\text { Buffer calculated percentage of land }(2,500 \mathrm{~m} \text { radius from hair snare cubby location) that is either } \\
\text { a natural grassland or a pasture for livestock. }\end{array}$ & $\psi$ & - \\
\hline PER_HIDEV & $\begin{array}{l}\text { Buffer calculated percentage of high intensity human development. This class is reserved } \\
\text { specifically for urban centers or areas of intense human usage. }\end{array}$ & $\psi$ & $-2,4,6$ \\
\hline PER_LOWDEV & $\begin{array}{l}\text { Buffer calculated percentage of low intensity human development. Common examples are minor } \\
\text { paved roads and single-family dwellings. }\end{array}$ & $\psi$ & $-2,4,6$ \\
\hline PER_MFOR & Percentage of mixed forest within a radius of $2,500 \mathrm{~m}$ of hair snare cubby location. & $\psi$ & $0^{5}$ \\
\hline REDO & $\begin{array}{l}\text { Categorical variable of whether or not a particular cell was run in } 2015 \text { and rerun in } 2016 \text {. The } \\
2016 \text { cells run in the same location as } 2015 \text { were coded with a } 1 \text { and all others } 0 \text {. }\end{array}$ & & \\
\hline ROAD_DEN & $\begin{array}{l}\text { Road density }\left(\mathrm{km} / \mathrm{km}^{2}\right) \text { of roads as calculated around pixel-meter }(30 \times 30) \text { raster cell at a radius } \\
\text { of 2,500m from the U.S. Census Bureau } 2015 \text { TIGER roads dataset. }\end{array}$ & $p, \psi$ & 2,6 \\
\hline SESSION & $\begin{array}{l}\text { A categorical variable with } 5 \text { classes that correspond to the } 4 \text {-week period, during which, each } \\
\text { location was sampled. More information on the session dates can be found in dissertation chapter } \\
\text { 2. Session } 1 \text { was encoded as zero to be compared with } 2,3,4 \text {, and } 5 \text {. }\end{array}$ & $p$ & - \\
\hline SIDI & $\begin{array}{l}\text { Simpson's Diversity Index, calculated for a 2,500m radius from hair snare cubby location. } \\
\text { Locations with more habitat types will receive a higher SIDI value. }\end{array}$ & $\psi$ & + \\
\hline YEAR & A categorical variable of whether a particular site was sampled in 2015 (0) or 2016 (1). & $p$ & 0 \\
\hline
\end{tabular}

${ }^{1}$ Clare et al. (2015), ${ }^{2}$ Lesmeister et al. (2015), ${ }^{3}$ Erb et al. (2012), ${ }^{4}$ Wang et al. (2015)

${ }^{5}$ McCord (1974), Litvaitis et al. (1986), Chamberlain et al. (1999, 2003), Long et al. (2011), Lesmeister et al.

(2015), ${ }^{6}$ Lombardi et al. (2017). 
Before covariate data collection occurred, three main a priori hypotheses were constructed to investigate which factors best explain bobcat $\psi$ in West Virginia at the landscape scale: (1) bobcat $\psi$ is a response to human disturbance, (2) bobcat $\psi$ is best explained as habitat selection for certain habitat types most associated with forested or contiguous environments, or (3) bobcat $\psi$ is a function of habitat selection for locations with more abundant or diverse prey. The covariates selected to model each hypothesis were thought to be the best descriptors out of the potential pool of all covariates. Each of these hypotheses were investigated for the samples collected in 2015, those from 2016, and a combined 2015-16 dataset to determine if the different sampled locations influenced the final conclusions of the study.

To model human disturbance of the landscape, the following covariates were selected to be run together: DST_UC, DST_SR, DST_PR, PER_HIDEV, ROAD_DEN, ELEV, and PER_AF (Table 3). It was hypothesized that bobcats would show a negative response to roads and development and a positive response to the percentage of forest in a buffer around the sampling locations.

To model bobcat habitat selection based on certain habitat characteristics, the following covariates were selected to be run together: PER_AF, PER_GRASS, DST_WA, PER_MFOR, PER_EFOR, PER_DFOR_2500, and LPI_DFOR (Table 3). Bobcats were hypothesized to show a positive response to the overall percentage of forest, but were not expected to select any particular location based on habitat type.

To model the final hypothesis, that the most important factors to bobcat $\psi$ were locations which had a prevalence or diversity of prey, the following covariates were used: MAST, ED, SIDI, DST_WA, and PER_GRASS (Table 3). It was hypothesized that areas with high edge or, diversity, or early successional forests offer the greatest amount of prey to bobcats and were 
most important factors in bobcat $\psi$ at the landscape scale. However, no early successional forest landcover type dataset exists so these covariates were used to surrogates for these types of habitats.

All modeling procedures were completed using the computer program R (R Core Team, 2013) package version of the program PRESENCE (Hines 2006), RPRESENCE (MacKenzie and Hines 2014). To model bobcat $\psi$ using these hypotheses, first, the factors impacting bobcat $p$ needed to be determined. The presence-absence data collected from the field were entered into the program using the following coding: for a week when no bobcats were detected at a site a 0 was entered. If a bobcat was detected at a particular site during a particular week, a 1 was entered. Thus, since each site was sampled for 4 weeks consecutively, a site at which a bobcat was detected during the second and last sampling occasion would be coded as 0101. Any sites for which samples could not be collected for a particular week were coded as a blank and were estimated by RPRESENCE based on covariate data.

The following covariates were selected to be modeled since they were thought to have the greatest impact on bobcat detection rates: SESSION, DST_TRL, DST_MR, DST_SR, PER_DFOR_1000, DST_UC, DST_PR, BearDAM, YEAR, and REDO (Table 3). To determine which of these variables were of greatest importance to bobcat $p$ all linear combinations of these covariates were run using the modCombos function of RPRESENCE, while holding $\psi$ constant as the dot model $\psi($.$) . However, the SESSION variables were included in each model since they$ were suspected to be of great importance due to the differential detection rates during each session. A total of 1,024 models were run for 2015, 2016, and 2015-16 to determine which variables were most important for $p$. Akaike’s Information Criterion (AIC) was used in model selection procedures to identify the models with the most evidence that had a $\triangle \mathrm{AIC} \geq 2$ 
(Burnham and Anderson 2002). The model for $p$ with the best evidence ratio was then held constant and used to run models for $\psi$ because no well-supported method exists for modelaveraging the $\beta$ values of $p$ (Clare et al. 2015, Lesmeister et al. 2015).

The same information-theoretic approach used for $p$ was employed to find the models with the greatest evidence ratios for $\psi$. For both the human avoidance hypothesis and habitat selection hypotheses, 128 unique models were run, and a total of 32 models were run for the prey habitat selection hypothesis. Models with a $\triangle \mathrm{AIC} \geq 2$ were considered to have sufficient evidence to be included in the final analyses completed for each of the datasets. To calculate the final predicted values of $\psi$ for each modeling situation, the modAvg function of RPRESENCE was used to combine the data from each of the selected models and create one final output.

Final interpolated maps were created for each dataset by overlaying the entirety of West Virginia with $10 \mathrm{~km}^{2}$ cells, just as were used for sampling sites in the field portion of this study. New covariate data were collected from the centroid points of these cells using the same methods previously described to be used in predictive modeling. Predicted values of $\psi$ were then applied to each cell in ArcGIS v.10.4 to create a predictive model. Finally, the Inverse Distance Weighted tool (IDW) was used to create an interpolated raster with a $1 \mathrm{~km}$ cell size of this dataset.

\section{Results}

Of the 2,014 hair samples collected in 2015, 1,641 were tested via qPCR, and 278 (13.8\%) were found to have originated from a bobcat. In 2016, a total of 2,108 hair samples were collected, 1,301 were tested via qPCR, of which 100 (4.7\%) were of bobcat origin. For both 2015 and 2016, a total of 21,000 trap nights of surveys were completed, since 30 grids of 25 cells 
were sampled four weeks each year. For 2015 an average bobcat detection rate of 1.3 bobcats per 100 trap nights was observed and for 2016 it was 0.47 bobcats per 100 trap nights. However, sites of marginal bobcat habitat were selected and sampled to gather covariates for this dataset, so the trap efficiency of the bobcat hair snare cubby in ideal bobcat habitat is probably much higher. Over the course of the two-year study a total of 1,275 unique sites were examined for bobcat presence or absence, 225 were visited twice, and a total of 378 bobcat detection events were recorded, for an overall bobcat detection rate of 0.9 detections per 100 trap nights.

\section{Occupancy Model}

From the 2015 candidate set of 1,024 bobcat models for $p$, only four had a $\Delta \mathrm{AIC} \leq 2$ and were selected for use in further analyses (Table 4). These four models were comprised of combinations of the variables DST_SR, DST_MR, DST_UC, SESSION, DST_TRL, and ROAD_DEN. Since no accepted method exists for averaging models of $p$ (Clare et al. 2015, Lesmeister et al. 2015), the detection model with the greatest evidence ratio used to construct the 2015 occupancy candidate models $(p$ DST_SR + DST_MR + DST_UC + SESSION, which is referred to as $p(\mathrm{BEST})$ was chosen. The estimates of $p(\mathrm{BEST})$ ranged from $0.037-0.389$ for each 1-week sampling period, with an overall mean $p$ of $0.133 \pm 0.027$, and an average sampling session $p$ of 0.532 . Of the $\beta$ estimates produced for $p$ (BEST), six had parameter estimates, when combined with the standard error, that did not cross zero, and were determined to be significant predictors for the model (Table 5). These covariates were DST_SR, DST_MR, DST_UC, SESSION 1, SESSION 2, and SESSION 5. DST_SR (-0.191 \pm 0.081$)$, DST_UC (-0.165 \pm $0.080)$, SESSION $1(-1.903 \pm 0.202)$, and SESSION 5 (-0.453 \pm 0.239$)$ were negatively correlated with $p$, while DST_MR $(0.191 \pm 0.073)$ and SESSION 2 (0.522 \pm 0.218$)$ were 
positively correlated with $p$. These results suggest that at the local scale, bobcat detection drops in proximity to nearby minor paved roads (DST_MR), yet detections are more likely in locations closer to secondary roads and urban clusters than completely undisturbed landscapes. The significance of the SESSION covariates document that as the sampling season progressed, $p$ did not remain the same.

Table 4 - Selected models for detection probability $(p)$ using bobcat detections recorded in West Virginia during the 2015 season. $-2 \mathrm{Ln} \mathrm{L}$ is 2 times the negative $\log$ likelihood of that particular model. $\Delta \mathrm{AIC}=$ Akaike's Information Criterion. $\omega r$ is the model weight ratio, recalculated for these selected models. $\mathrm{K}^{*}$ is the number of parameters included in the model. This number is greater than the number of parameters listed under model because SESSION has to be coded as four separate variables (SESSION 1 was reference and absorbed into the intercept and SESSIONS 2, 3, 4, and 5 were included) in RPRESENCE. (For covariate definitions, see Table 3).

\begin{tabular}{|c|c|c|c|c|c|}
\hline & Model & $-2 \operatorname{Ln} L$ & $\Delta \mathrm{AIC}$ & $\omega^{\mathrm{r}}$ & $\mathrm{K}^{*}$ \\
\hline 1 & $\psi \sim \underset{\text { SESSION }}{\psi \sim 1, p \sim \text { DST_SR + DST_MR + DST_UC + }}+$ & 1800.38 & 0.00 & 0.41 & 9 \\
\hline 2 & $\begin{array}{c}\psi \sim 1, p \sim \text { DST_SR + DST_MR + PER_DFOR_1000 } \\
\text { +DST_UC + SESSION }\end{array}$ & 1799.18 & 0.80 & 0.27 & 10 \\
\hline 3 & $\begin{array}{c}\psi \sim 1, p \sim \text { DST_SR + DST_UC + DST_MR + } \\
\text { DST_TRL + SESSION }\end{array}$ & 1800.21 & 1.83 & 0.16 & 10 \\
\hline 4 & $\begin{array}{c}\psi \sim 1, p \sim \text { DST_SR + DST_UC + DST_MR + } \\
\text { ROAD_DEN + SESSION }\end{array}$ & 1800.37 & 1.99 & 0.15 & 10 \\
\hline
\end{tabular}

Table 5 - $\beta$ values (with standard errors) for the BEST model for detection probability ( $p$ ) of the 2015 dataset and the included covariates (Table 3). This was then held constant when modeling occupancy ( $\psi$ ) for the 2015 dataset. Values in bold indicate that the parameter estimate, when combined with the standard error, did not cross zero, and is a significant predictor.

\begin{tabular}{|c|c|c|}
\hline & Model & $\psi \sim 1, p \sim$ DST_SR + DST_MR + DST_UC + SESSION \\
\hline \multirow{8}{*}{ 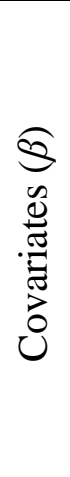 } & DST_SR & $-0.191 \pm 0.081$ \\
\hline & DST_MR & $0.191 \pm 0.073$ \\
\hline & DST_UC & $-0.165 \pm 0.080$ \\
\hline & SESSION 1 & $-1.903 \pm 0.202$ \\
\hline & SESSION 2 & $0.522 \pm 0.218$ \\
\hline & SESSION 3 & $-0.204 \pm 0.236$ \\
\hline & SESSION 4 & $-0.070 \pm 0.246$ \\
\hline & SESSION 5 & $-0.453 \pm 0.239$ \\
\hline
\end{tabular}


Of the 288 model candidate pool for $\psi, 13$ models with a $\Delta \mathrm{AIC} \leq 2$ were selected for use in further analyses (Table 6). The covariates that comprised these 13 models only related to those describing the level of human impacts on a particular landscape. Each of the seven covariates chosen to model the human impact hypothesis appeared in at least one of the selected models. The 13 selected models were then averaged to produce the final 2015 occupancy model. The model averaged estimates of $\psi$ ranged from $0.005-1.000$, with a mean $\psi$ of $0.858 \pm 0.057$. The 2015 occupancy model predicts very high levels of bobcat occupancy along the Appalachian Mountain corridor running through the center of the state and moderate to low levels of occupancy in the western portion and eastern panhandle (Figures 5, 6). Cells located within or in close proximity to urban areas have very low predicted values of $\psi$.

The values of $\beta$ for each model were not averaged together since no consensus method exists for completing this procedure on covariates not present in every selected model (Clare et al. 2015). Each covariate used in every model of $\psi$ for 2015 was considered to be significant because the estimates, when combined with the standard error, did not cross zero (Table 7). The covariates appearing in the most models, and thus suspected of being the best predictors of $\psi$ at the landscape scale, were ELEV and ROAD_DEN. ELEV was positively correlated with $\psi$, indicating that bobcats are more likely to inhabit sites at higher elevations where overall human impacts may be less. ROAD_DEN was negatively correlated with $\psi$, which means that bobcats were more likely to occupy sites with lower road densities. While these were the most significant predictors, $\psi$ was also negatively correlated with PER_AF and DST_UC, indicating that bobcats were more likely to occupy locations with less percentage total forest and that were closer to urban clusters. The mixture of both positive and negative correlations with certain covariates of 
human impact suggest that bobcats may have different responses to human disturbance at varying spatial scales. 
Table 6 - Selected models for the probability of occupancy $(\psi)$ and the 2015 dataset of bobcat detections in West Virginia. -2 Ln L is 2 times the negative log likelihood of that particular model. $\triangle \mathrm{AIC}=$ Akaike's Information Criterion. $\omega \mathrm{r}$ is the model weight ratio, recalculated for these selected models. $\mathrm{K}^{*}$ is the number of parameters (Table 3) included in the model. This number is greater than the number of parameters listed under model because SESSION has to be coded as four separate variables (SESSION 1 was reference and absorbed into the intercept and SESSIONS 2, 3, 4, and 5 were included) in RPRESENCE.

\begin{tabular}{|c|c|c|c|c|c|}
\hline & Model & $-2 \operatorname{Ln} L$ & $\triangle \mathrm{AIC}$ & $\omega^{\mathrm{r}}$ & $\mathrm{K}^{*}$ \\
\hline 1 & $\psi \sim$ DST_UC + PER_HIDEV + ROAD_DEN + ELEV + PER_AF, $p \sim($ BEST) & 1770.35 & 0.00 & 0.149 & 14 \\
\hline 2 & $\psi \sim$ DST_SR + ROAD_DEN + ELEV + PER_AF, $p \sim($ BEST) & 1773.45 & 1.11 & 0.086 & 13 \\
\hline 3 & $\psi \sim$ DST_SR + DST_PR + ROAD_DEN + ELEV + PER_AF, $p \sim(\mathrm{BEST})$ & 1771.48 & 1.13 & 0.085 & 14 \\
\hline 4 & $\psi \sim$ DST_UC + DST_PR + PER_HIDEV + ROAD_DEN + ELEV + PER_AF, $p \sim($ BEST) & 1769.62 & 1.28 & 0.079 & 15 \\
\hline 5 & $\psi \sim$ DST_UC + ROAD_DEN + ELEV + PER_AF, $p \sim(\mathrm{BEST})$ & 1773.64 & 1.29 & 0.078 & 13 \\
\hline 6 & $\psi \sim$ DST_UC + DST_SR + ROAD_DEN + ELEV + PER_AF, $p \sim($ BEST) & 1771.70 & 1.35 & 0.076 & 14 \\
\hline 7 & $\psi \sim$ DST_UC + ROAD_DEN + ELEV,$p \sim(\mathrm{BEST})$ & 1775.88 & 1.53 & 0.069 & 12 \\
\hline 8 & $\psi \sim$ DST_UC + DST_PR + ROAD_DEN + ELEV,$p \sim(\mathrm{BEST})$ & 1773.91 & 1.56 & 0.068 & 13 \\
\hline 9 & $\psi \sim$ ELEV + DST_SR + PER_HIDEV+ROAD_DEN + ELEV + PER_AF, $p \sim($ BEST) & 1772.00 & 1.66 & 0.065 & 14 \\
\hline 10 & $\psi \sim$ DST_SR + PER_HIDEV + ROAD_DEN + ELEV + PER_AF, $p \sim($ BEST $)$ & 1772.00 & 1.66 & 0.065 & 14 \\
\hline 11 & $\psi \sim \mathrm{DST} \_\mathrm{UC}+\mathrm{DST} \_\mathrm{SR}+\mathrm{DST} \_\mathrm{PR}+\mathrm{PER} \_\mathrm{HIDEV}+\mathrm{ROAD}$ DEN + ELEV + PER_AF, $p \sim(\mathrm{BEST})$ & 1768.08 & 1.73 & 0.063 & 16 \\
\hline 12 & $\psi \sim$ DST_UC + DST_SR + DST_PR + ROAD_DEN + ELEV + PER_AF, $p \sim($ BEST) & 1770.17 & 1.83 & 0.06 & 15 \\
\hline 13 & $\psi \sim$ DST_UC + DST_SR + DST_PR + ROAD_DEN + ELEV,$p \sim(\mathrm{BEST})$ & 1772.19 & 1.84 & 0.059 & 14 \\
\hline
\end{tabular}


Table $7-\beta$ values (with standard errors) for the selected models for occupancy probability ( $\psi$ ) of the 2015 West Virginia bobcat detection dataset and the included covariates (Table 3). Values in bold indicate that the parameter estimate, when combined with the standard error, did not cross zero, and is a significant predictor. F is the number of selected models in which that covariate is a significant predictor.

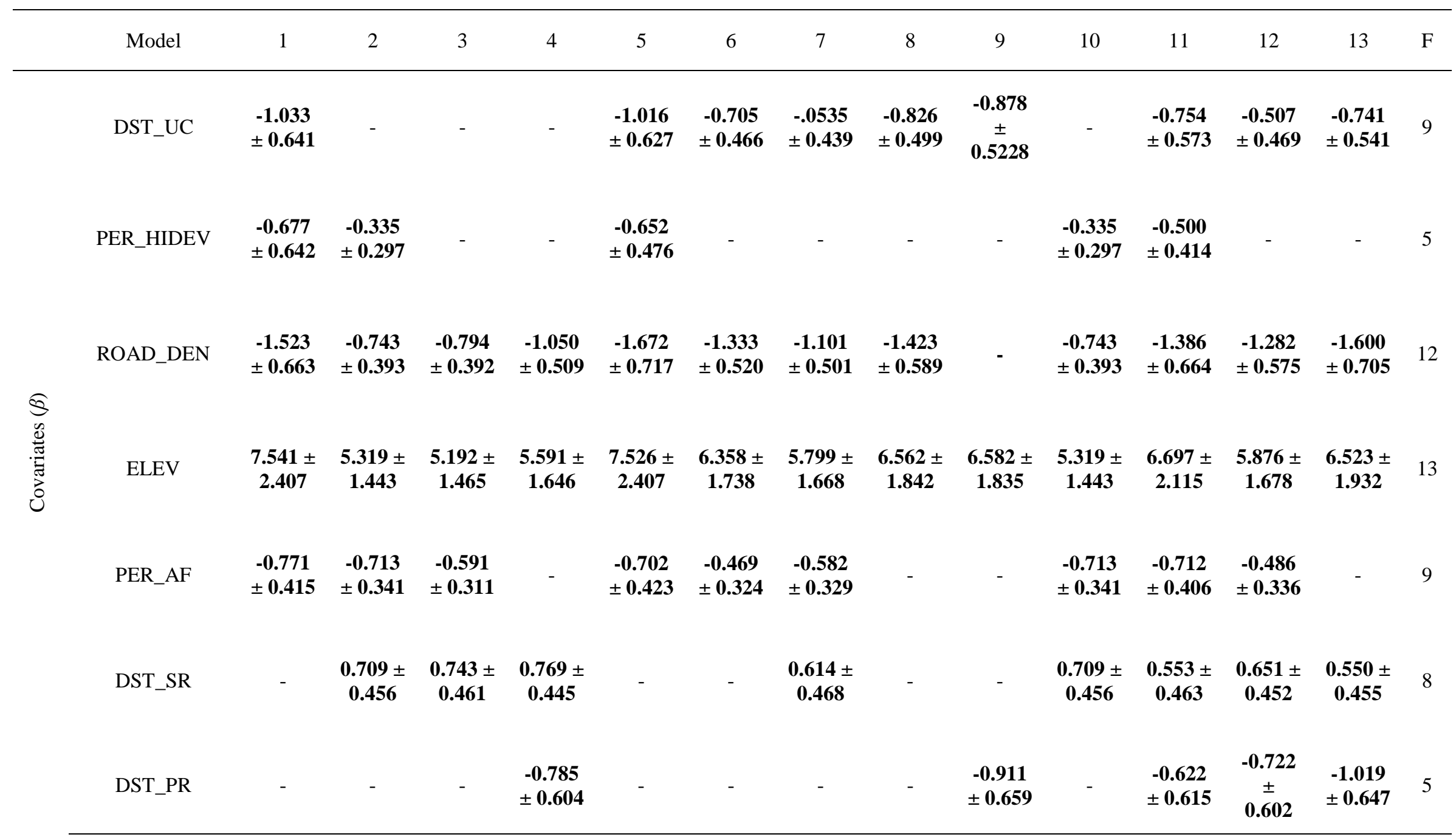




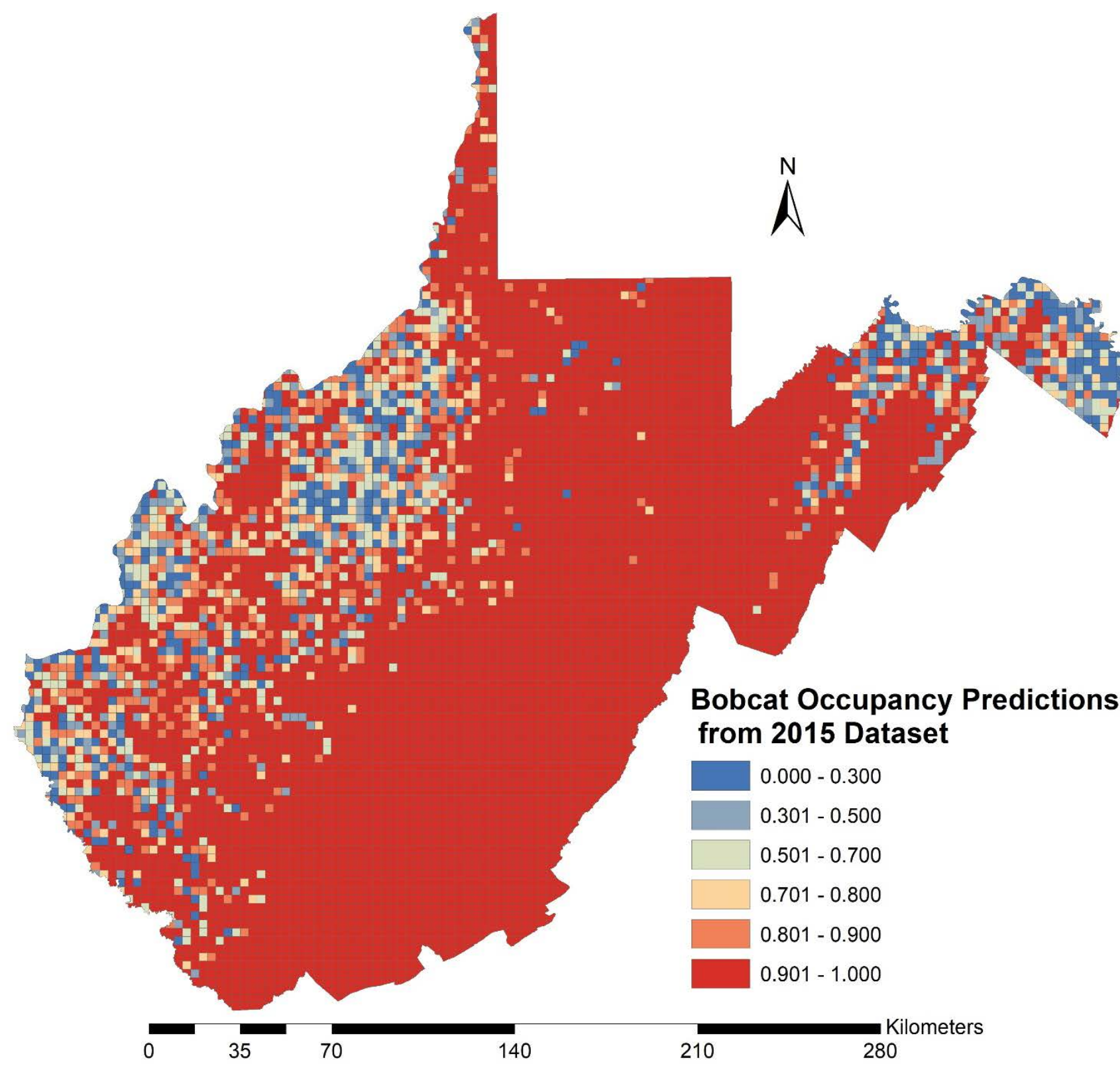

Figure 5 - A 10 $\mathrm{km}^{2}$ raster of West Virginia with pixels assigned the values of bobcat occupancy probability $(\psi)$ as predicted from the model-averaged 2015 occupancy model for covariates collected from centroid points of each polygon. 


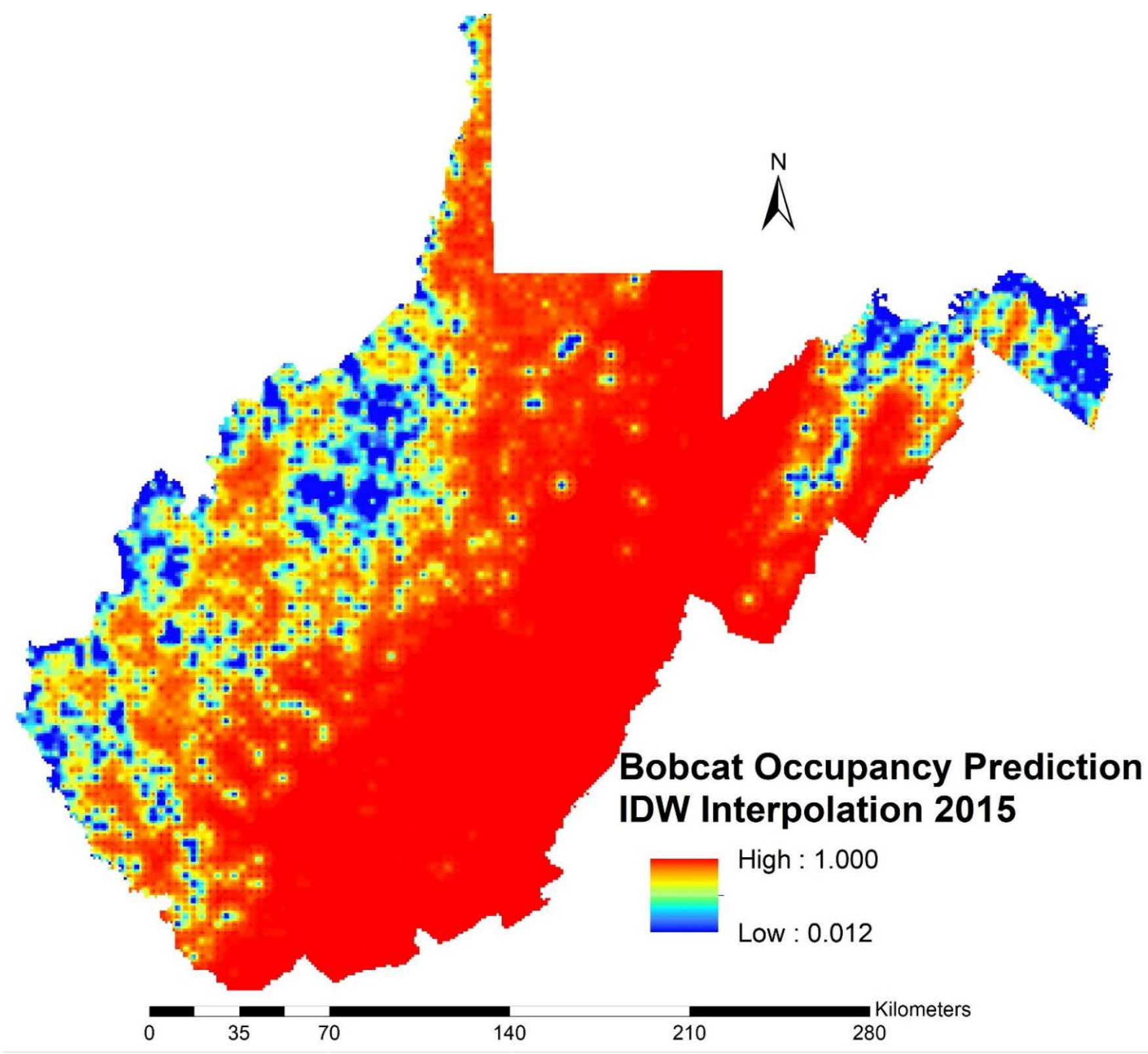

Figure 6 - Inverse Distance Weighted (IDW) interpolated raster created using the values of bobcat occupancy probability ( $\psi$ ) predicted for each raster cell by the 2015 occupancy model. 


\section{Occupancy Model}

Of the 1,024 models of $p$ examined for 2016, more than $80 \%$ failed to converge, and for those that converged, the standard errors of parameter estimates were 0.000 . These results are indicative of an underlying problem with this dataset. These issues were most likely a result of the sparseness of the 2016 dataset, when considered on its own, since only 100 bobcat detections were recorded. This problem is not uncommon in occupancy modeling when a dataset does not have sufficient statistical power to discern amongst covariates when the dataset used to initially estimate parameters is limited. This exact problem was encountered by Lesmeister et al. (2015) when examining the camera-cluster scale data when including certain covariates. Due to the high probability of constructing an erroneous occupancy model, the 2016 dataset, on its own, was not used for any additional analyses.

\section{5-16 Occupancy Model}

Of the 1,024 candidate models examined for $p$ for the 2015-16 dataset, 12 had a $\Delta \mathrm{AIC} \leq$ 2 (Table 8). However, only the top detection model was used for further analyses, as described previously, and was held as a constant for use in modeling $\psi(p \sim$ DST_MR + YEAR + REDO + SESSION). The estimates of $p$ for the best model ranged from $0.031-0.241$, with a mean of $0.098 \pm 0.014$, and a per-session average $p$ of 0.392 . The $\beta$ values for the 8 covariates included in $p(\mathrm{BEST})$ were all found to be important to the model since the parameter estimate of each covariate, combined with their standard error, did not cross zero (Table 9). DST_MR (0.117 \pm 0.056) and SESSION 2 (0.231) were positively correlated with $p$, while YEAR (-0.932 \pm 0.140$)$, REDO (-0.763 \pm 0.272$)$, SESSION 1 (-1.617 \pm 0.177$)$, SESSION $3(-3.53 \pm 0.186)$, SESSION 4 $(-0.523 \pm 0.192)$, and SESSION $5(-0.656 \pm 0.200)$ were negatively correlated with $p$. Just as was 
in the 2015 dataset, bobcat $p$ was reduced in close proximity to minor roads. Again, $p$ was not constant across the sampling sessions and was greatest in SESSION 2. Bobcat $p$ was also reduced in 2016, as compared to 2015, and $p$ was also less in 2016 at the same sites that were also sampled in 2015, suggesting a potential trap-aversion response of previously exposed bobcats. 
Table 8 - Selected models for detection probability ( $p$ ) using the 2015-16 dataset of bobcat detections in West Virginia. $-2 \mathrm{Ln} \mathrm{L}$ is 2 times the negative log likelihood of that particular model. $\Delta \mathrm{AIC}=$ Akaike's Information Criterion. $\omega r$ is the model weight ratio, recalculated for these selected models. $\mathrm{K}^{*}$ is the number of parameters (Table 3) included in the model. This number is greater than the number of parameters listed under model because SESSION has to be coded as four separate variables (SESSION 1 was reference and absorbed into the intercept and SESSIONS 2, 3, 4, and 5 were included) in RPRESENCE.

\begin{tabular}{|c|c|c|c|c|c|}
\hline & Model & $-2 \operatorname{Ln} L$ & $\Delta \mathrm{AIC}$ & $\omega$ & $\mathrm{K}$ \\
\hline 1 & $p \sim$ DST_MR + YEAR + REDO + SESSION & 2700.97 & 0.00 & 0.08 & 9 \\
\hline 2 & $p \sim$ DST_PR + YEAR + REDO + SESSION & 2701.21 & 0.23 & 0.07 & 9 \\
\hline 3 & $\begin{array}{c}p \sim \text { DST_MR + PER_DFOR_1000 + YEAR + REDO + } \\
\text { SESSION }\end{array}$ & 2699.51 & 0.54 & 0.06 & 10 \\
\hline 4 & $p \sim$ DST_MR + DST_PR + YEAR + REDO + SESSION & 2699.54 & 0.57 & 0.06 & 10 \\
\hline 5 & $p \sim$ DST_MR + BearDAM + YEAR + REDO + SESSION & 2699.58 & 0.61 & 0.06 & 10 \\
\hline 6 & $p \sim \mathrm{YEAR}+\mathrm{REDO}+\mathrm{SESSION}$ & 2703.60 & 0.63 & 0.06 & 8 \\
\hline 7 & $p \sim$ DST_UC + DST_PR + YEAR + REDO + SESSION & 2699.75 & 0.78 & 0.06 & 10 \\
\hline 8 & $p \sim$ DST_MR + DST_PR + BearDAM + YEAR + REDO + & 2697.83 & 0.86 & 0.05 & 11 \\
\hline 9 & $p \sim$ DST_PR + BearDAM + YEAR + REDO + SESSION & 2700.03 & 1.06 & 0.05 & 10 \\
\hline 10 & $p \sim$ DST_MR + DST_UC + DST_PR + YEAR + REDO + & 2698.19 & 1.21 & 0.05 & 11 \\
\hline 11 & $\begin{array}{c}p \sim \text { DST_MR + PER_DFOR_1000 + BearDAM + YEAR + } \\
\text { REDO + SESSION }\end{array}$ & 2698.33 & 1.36 & 0.04 & 11 \\
\hline 12 & $\begin{array}{c}p \sim \text { DST_MR + PER_DFOR_1000 + DST_PR + YEAR + } \\
\text { REDO + SESSION }\end{array}$ & 2698.35 & 1.37 & 0.04 & 11 \\
\hline 13 & $\begin{array}{c}p \sim \text { PER_DFOR_1000 + DST_PR + YEAR + REDO + } \\
\text { SESSION }\end{array}$ & 2700.59 & 1.62 & 0.04 & 10 \\
\hline 14 & $p \sim$ DST_MR + DST_SR + YEAR + REDO + SESSION & 2700.6 & 1.62 & 0.04 & 10 \\
\hline 15 & $p \sim$ DST_SR + DST_PR + YEAR + REDO + SESSION & 2700.64 & 1.67 & 0.04 & 10 \\
\hline 16 & $\begin{array}{c}p \sim \text { DST_MR + DST_UC + DST_PR + BearDAM + YEAR + } \\
\text { REDO + SESSION }\end{array}$ & 2696.83 & 1.86 & 0.03 & 12 \\
\hline 17 & $p \sim$ PER_DFOR_1000 + YEAR + REDO + SESSION & 2702.85 & 1.88 & 0.03 & 9 \\
\hline 18 & $p \sim$ DST_MR + DST_UC + YEAR + REDO + SESSION & 2700.85 & 1.88 & 0.03 & 10 \\
\hline 19 & $\begin{array}{c}p \sim \text { DST_UC + DST_PR + BearDAM + YEAR + REDO + } \\
\text { SESSION }\end{array}$ & 2698.86 & 1.89 & 0.03 & 11 \\
\hline 20 & $p \sim$ BearDAM + YEAR + REDO + SESSION & 2702.86 & 1.89 & 0.03 & 10 \\
\hline 21 & $\begin{array}{c}p \sim \text { DST_MR + PER_DFOR_1000 + DST_PR + BearDAM + } \\
\text { YEAR + REDO + SESSION }\end{array}$ & 2696.88 & 1.91 & 0.03 & 12 \\
\hline
\end{tabular}


Table 9 - $\beta$ values (with standard errors) for the BEST model for detection probability ( $p$ ) of the 2015-16 dataset and included covariates (Table 3). This was then held constant when modeling the probability of occupancy ( $\psi$ ) for the 2015-16 West Virginia bobcat presence-absence dataset. Values in bold indicate that the parameter estimate, when combined with the standard error, did not cross zero, and is a significant predictor.

\begin{tabular}{|c|c|c|}
\hline & Model & $p \sim$ DST_MR + YEAR + REDO + SESSION \\
\hline \multirow{8}{*}{ 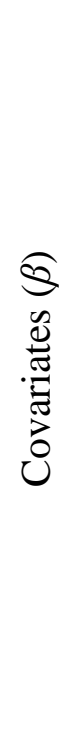 } & DST_MR & $0.117 \pm 0.056$ \\
\hline & YEAR & $-0.932 \pm 0.140$ \\
\hline & REDO & $-0.763 \pm 0.272$ \\
\hline & SESSION 1 & $-1.617 \pm 0.177$ \\
\hline & SESSION 2 & $0.231 \pm 0.172$ \\
\hline & SESSION 3 & $-0.353 \pm 0.186$ \\
\hline & SESSION 4 & $-0.523 \pm 0.192$ \\
\hline & SESSION 5 & $-0.656 \pm 0.200$ \\
\hline
\end{tabular}

From the 288 candidate models constructed for $\psi$ using the 2015-16 dataset, a total of five models were selected and included in further analyses ( $\triangle \mathrm{AIC} \leq 2)$ (Table 10). The five models were comprised of combinations of all seven covariates included in the original hypotheses to describe the varying levels of human impact on the landscape. The model averaged estimates of $\psi$ ranged from $0.062-1.000$, with a mean $\psi$ of $0.834 \pm 0.055$. The 201516 occupancy model predicts very high levels of bobcat occupancy throughout the Appalachian Mountain corridor running through the center of the state, just as the 2015 model did (Figures 7, 8). However, the western portion of the state and eastern panhandle appear to have lower overall predicted bobcat occupancies. This is most likely as a result of the greater number of nondetections in the 2016 dataset, particularly in the western portions of the state. Cells located within or in close proximity to urban areas have reduced predicted occupancy, just as was found 
in 2015. Just as was found using only the 2015 - 2016 dataset, bobcats are modeled to have a statewide distribution.

The $\beta$ values calculated for each model of the 2015-16 dataset were not averaged together, just as described in the previous section. Of the seven covariates included in the selected five models, only four were found to be significant because their parameter estimates combined with standard errors did not cross zero (Table 11). ROAD_DEN, ELEV, and PER_AF were included in all five of the selected models with DST_PR was only in four. A positive relationship between $\psi$ was documented for ELEV, indicating that, generally, bobcats preferred locations at higher elevations. DST_PR, PER_AF, and ROAD_DEN were negatively correlated with $\psi$, meaning that bobcats selected locations in closer overall proximity to larger roads, but avoided locations with high road density. Also, bobcats were more likely to occur in locations with lower percentages of total forest, indicating that bobcats favored areas with some level of human disturbance. 
Table 10 - Selected models for occupancy probability $(\psi)$ using the 2015-16 West Virginia bobcat presence-absence dataset. -2 Ln L is 2 times the negative log likelihood of that particular model. $\triangle \mathrm{AIC}=$ Akaike's Information Criterion. $\omega$ r is the model weight ratio, recalculated for these selected models. $\mathrm{K} *$ is the number of parameters (Table 3) included in the model. This number is greater than the number of parameters listed under model because SESSION has to be coded as four separate variables (SESSION 1 was reference and absorbed into the intercept and SESSIONS 2, 3, 4, and 5 were included) in RPRESENCE.

\begin{tabular}{|c|c|c|c|c|c|}
\hline & Model & $-2 \operatorname{Ln} L$ & $\triangle \mathrm{AIC}$ & $\omega^{\mathrm{r}}$ & $\mathrm{K}$ \\
\hline 2 & $\psi \sim$ DST_UC + DST_PR + ROAD_DEN + ELEV + PER_AF,$p \sim($ BEST $)$ & 2641.98 & 1.55 & 0.18 & 14 \\
\hline 3 & $\psi \sim$ ROAD_DEN + ELEV + PER_AF, $p \sim($ BEST) & 2646.27 & 1.84 & 0.15 & 12 \\
\hline 4 & $\psi \sim$ DST_SR + DST_PR + ROAD_DEN + ELEV + PER_AF,$p \sim(\mathrm{BEST})$ & 2642.41 & 1.98 & 0.14 & 14 \\
\hline
\end{tabular}

Table 11 - $\beta$ values (with standard errors) for the BEST model for probability of detection ( $p$ ) of the 2015-16 dataset of West Virginia bobcat presence-absence and the included covariates (Table 3). This was then held constant when modeling $\psi$ for the 2015-16 dataset. Values in bold indicate that the parameter estimate, when combined with the standard error, did not cross zero, and is a significant predictor.

\begin{tabular}{|c|c|c|c|c|c|c|c|}
\hline & Model & 1 & 2 & 3 & 4 & 5 & $\mathrm{~F}$ \\
\hline \multirow{7}{*}{ 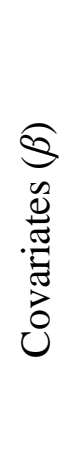 } & DST_PR & $-0.856 \pm 0.453$ & $-0.791 \pm 0.446$ & & $-0.856 \pm 0.452$ & $-0.856 \pm 0.454$ & 4 \\
\hline & ROAD_DEN & $-0.837 \pm 0.361$ & $-0.842 \pm 0.350$ & $-0.582 \pm 0.299$ & $-0.830 \pm 0.364$ & $-0.838 \pm 0.369$ & 5 \\
\hline & ELEV & $4.798 \pm 1.431$ & $4.816 \pm 1.385$ & $4.328 \pm 1.447$ & $4.792 \pm 1.429$ & $4.799 \pm 1.438$ & 5 \\
\hline & PER_AF & $-0.748 \pm 0.432$ & $-0.647 \pm 0.426$ & $-0.760 \pm 0.442$ & $-0.750 \pm 0.434$ & $-0.747 \pm 0.458$ & 5 \\
\hline & DST_UC & - & $-0.214 \pm 0.319$ & - & - & - & 0 \\
\hline & DST_SR & - & - & - & $0.042 \pm 0.326$ & - & 0 \\
\hline & PER_HIDEV & - & - & - & - & $0.003 \pm 0.336$ & 0 \\
\hline
\end{tabular}




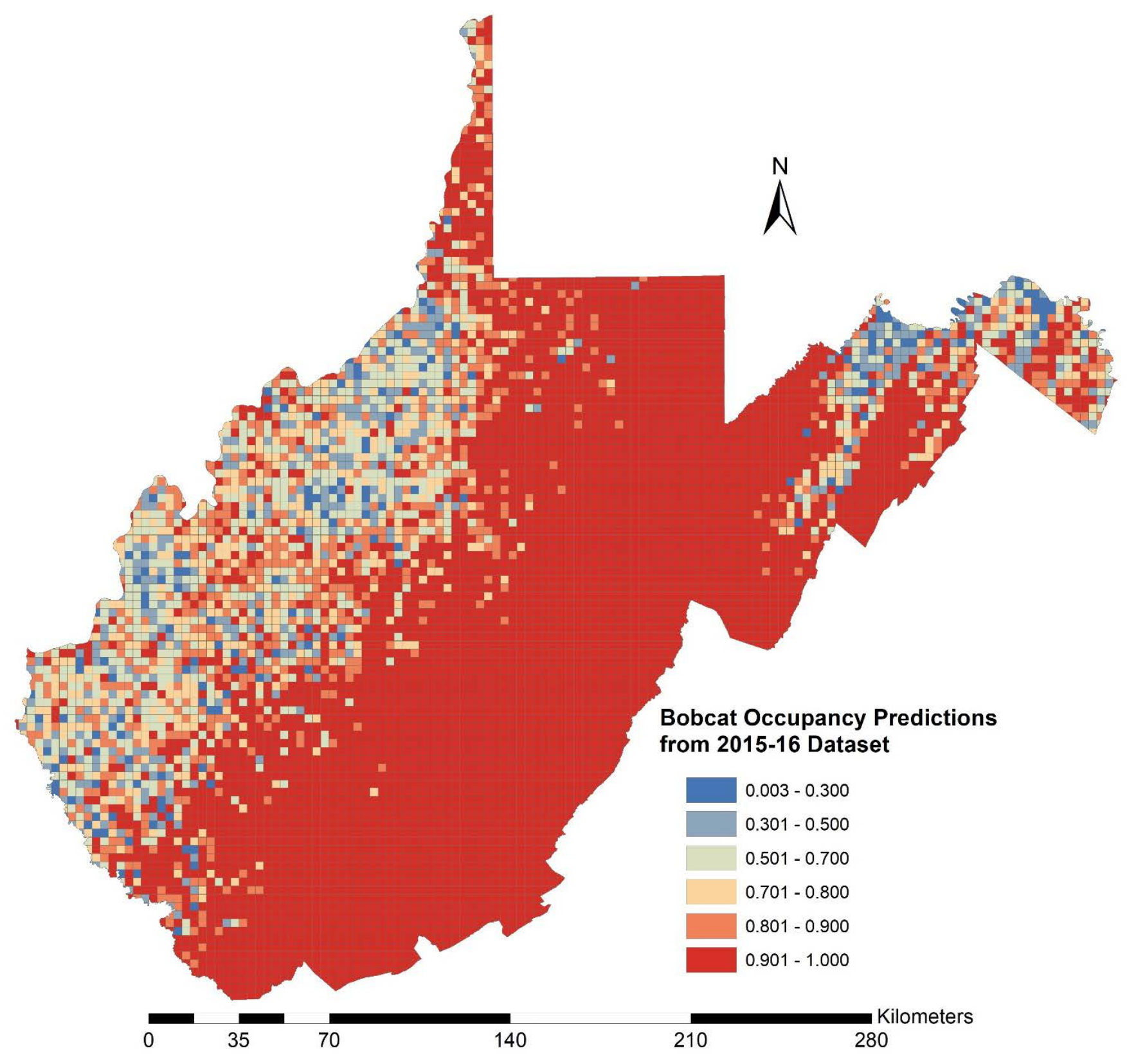

Figure 7 - A 10km² raster of West Virginia with pixels assigned the values of the probability of bobcat occupancy ( $\psi$ ) as predicted from the 2015-16 occupancy model for the covariates collected from centroid points of each polygon. 


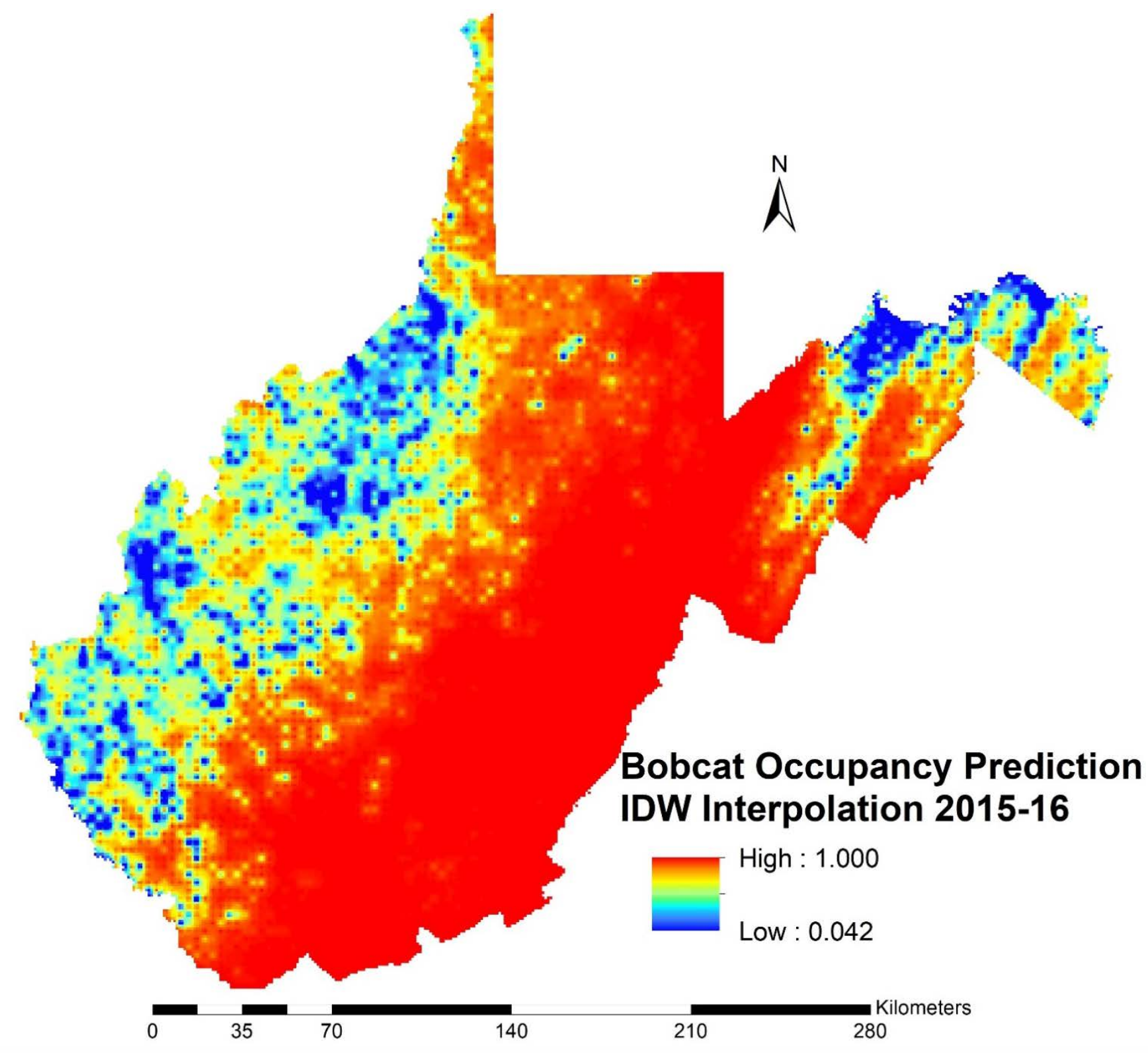

Figure 8 - Inverse Distance Weighted (IDW) interpolated raster created using the values of predicted bobcat occupancy probability $(\psi)$ for each raster cell by the 2015-16 occupancy model. 


\section{Discussion}

\section{Predicted Estimates of Detection and Occupancy at the Landscape Scale}

The average predicted bobcat $\psi$ was $0.858 \pm 0.057$ and $0.834 \pm 0.055$, for the 2015 and 2015-16 datasets, respectively. As was expected with a continuously-ranging mesocarnivore species like the bobcat, the average $\psi$ is relatively high, indicating that bobcats likely inhabit most acceptable habitats that are currently available in West Virginia. Occupancy is either 1, or very close to 1, along the heavily forested core in and surrounding the Appalachian Mountains and Monongahela National Forest, and is reduced heavily in the more populated areas along the Ohio River and the far eastern panhandle. These predicted average values of $\psi$ are similar to what was found by Lesmeister et al. (2015) in a camera-trapping survey in southern Illinois (0.75), but much higher than the survey of Clare et al. (2015) in central Wisconsin (0.40), Erb et al. (2012) along the Appalachian Trail corridor (0.32), and Long et al. (2011) in Vermont (0.326). A direct comparison of these modeled occupancy values suggests that bobcats are much more ubiquitous in West Virginia than most of these other sampled locations.

The average predicted bobcat $p$ was $0.133 \pm 0.027$ and $0.098 \pm 0.014$, for the 2015 and the 2015-16 datasets, respectively. These values are within what would be expected for a highly mobile, yet elusive species that can be difficult to sample. Over the course of a session, each individual cell was predicted to have a $53.2 \%$ or $39.2 \%$ probability of successfully sampling a bobcat, if one was present in the sampling cell, for the 2015 and 2015-16 datasets, respectively. The average predicted weekly $p$ from this study is similar to what was found using camera traps in other locations, such as Erb et al. (2012) along the Appalachian Trail corridor (0.098). However, the single-week estimate of $p$ was lower than other camera-trapping studies in central Wisconsin (Clare et al. 2015) (0.216), southern Illinois (Lesmeister et al. 2015) (0.20), and a scat 
detection dog survey in Vermont (Long et al. 2011) (0.27). This study is the first in recent years to complete large-scale occupancy modeling using a felid hair-snare so a direct comparison to other studies cannot be made. Hair snares are assumed to be less effective at sampling present animals, as compared to camera-trapping, because an interaction between the animal to be sampled and the device is required. However, many of these studies were completed in locations with much more defined travel routes between areas of acceptable bobcat habitat that is surrounded by mediocre habitat. As such, these travel routes may artificially increase the calculated value of $p$, since habitat features like these are uncommon in West Virginia, due to the complex topography and the well above-average percentage of forested overstory. However, the calculated values of $p$ from this study still remain comparable to those of camera studies. The success of the hair snare cubby should be evaluated in additional localities to gain a better understanding of the strengths and weaknesses of the device in diverse settings.

\section{Best Covariates for Explaining Differences in Probability of Detection}

For the 2015 dataset, the most important covariates in regards to predicting $p$ were the human impact covariates of DST_SR (distance to secondary road), DST_MR (distance to minor road), DST_UC (distance to urban cluster), and the sampling covariates of SESSIONS 1, 2, and 5. DST_SR and DST_UC were both negatively correlated with bobcat $p$, indicating that bobcat detections were negatively impacted by the distance to a secondary road or urban cluster, and were higher in close proximity to these features. However, bobcat $p$ was positively correlated with DST_MR, meaning that bobcat detections increased as the distance to a minor road also increased. The relationships of these variables to bobcat $p$ suggest that bobcat detection is affected by human impact at different spatial scales. At the local scale, bobcats avoid minor 
paved roads and were more likely to be detected in locations not adjacent to these roads.

However, at the larger spatial scale, bobcats were more likely to be detected in areas with some level of human impact, such as the proximity of secondary or primary roads. Perhaps bobcats moved around the landscape more in these disturbed locations and were thus more likely to be exposed to the sampling devices. Erb et al. (2012), Clare et al. (2015), and Lewis et al. (2015) used similar human disturbance covariates in their studies that were also found to be negatively correlated with bobcat $p$.

Finally, the session in which the sampling took place was found to significantly impact $p$. The probability of detection was greatest during SESSION 2, which took place from April through early May (Table 1), appears to be a time when overall bobcat movement is the greatest, as the juveniles leave the home ranges of their mothers and disperse to search for an unoccupied home range (Kitchings and Story 1984). However, it is also possible that the locations with the greatest densities of bobcats, which would artificially increase $p$, were all coincidentally sampled during SESSION 2, or that the nearly three-week difference in the session start date between 2015 and 2016 created a confounding response. SESSIONS 1 and 5 had significant negative $\beta$ 's $(-1.903,-0.453)$ indicating that at these times of the year, bobcat detections were less than what was expected based on what was found during SESSION 2. Future studies may want to avoid sampling during SESSIONS 1 and 5, which are times when the same level of sampling effort will yield significantly fewer bobcat detections, as compared to SESSIONS 2, 3, and 4 (April June). While included as a potential covariate for the models of $p$, BearDAM (bear damage) was not in the best supported during the model selecting process, and thus was not included in construction of the final model. While bears damaging the sampling stations may have had an 
impact on the overall collection of bobcat samples, this impact was not so significant that it stood out beyond other covariates during the modeling procedures.

For the combined 2015-16 dataset, the most important covariates that impacted $p$ were the human impact covariates of DST_MR (distance to minor road), YEAR, REDO, and the sampling covariates of SESSIONS 1, 2, 3, 4, and 5. DST_MR was found again to be positively correlated to the distance to minor roads indicating that as the distance to minor paved roads became greater, so did bobcat $p$. This suggests that bobcats are likely to use areas adjacent to roads less frequently. Both YEAR and REDO were found to be negatively correlated with bobcat detections. Bobcat detections much lower at sites sampled in 2016 than in 2015, and was also lower at sites that were sampled in 2015 and redone in 2016. This result is important because it documents a drop in detections when resampling the same areas, or that the recapture success of the bobcat hair snare may be limited, even when $p$ is relatively high for a hair snare and naïve encounters. The same patterns of the impact of particular sessions were also observed in the 2015-16 dataset in that the best detection rates occurred during SESSIONS 2, 3, and 4, with SESSIONS 1 and 5, having lower predicted values of $p$.

\section{Best Covariates for Explaining Variances in Bobcat Occupancy Probability}

Through model selection, it was determined that the most important factors determining bobcat $\psi$ in West Virginia are those associated with human impacts-for both the 2015 and the 2015-2016 datasets. Each of the seven human impact covariates tested was found in at least one model with a $\triangle \mathrm{AIC} \geq 2$ and were a part of the final averaged model. However, since there is no consensus method on model averaging $\beta$ estimates for parameters (Clare et al. 2015), this was not completed, and the following discussion will be of trends discovered in the $\beta$ 's from multiple 
models. For the 2015 dataset, the covariates of ELEV and ROAD_DEN appeared in 13 and 12 of the final 13 selected models, respectively, and were considered to be the most important variables predicting bobcat occupancy. Elevation was a strong positive predictor of bobcat $\psi$, presumably because human impacts are greatest in the many valleys of West Virginia and areas of higher elevation are often less disturbed and offer more contiguous habitat for bobcats. Road density was another strong predictor of bobcat $\psi$, but with a negative relationship, meaning that bobcat $\psi$ was reduced in areas with more roads. A similar conclusion was also reached by Lesmeister et al. (2015), as roads were also some of the most important predictors of bobcat $\psi$ in their study.

Other moderate predictors of bobcat $\psi$ for 2015 were DST_UC (distance to urban cluster), PER_AF (percentage all forest), and DST_SR (distance to secondary road). Bobcat $\psi$, was negatively correlated with DST_UC and PER_AF. Bobcats were more likely to occupy areas closer to urban clusters than not and they also selectively occupied areas with a lower percentage of total forest (mean forest percentage for studied sites was roughly 80\%). The response of bobcat $\psi$ to these covariates is likely the result of their selection of habitats with some form of disturbance near urban clusters that creates early successional habitats to increase prey diversity and density. Previous studies have found that bobcats are not particularly associated with certain habitat types, but choose their home ranges based on prey density (McCord 1974, Litvaitis et al. 1986, Chamberlain et al. 1999, 2003, Long et al. 2011, Lesmeister et al. 2015). While a prey selection hypothesis was included in this study, the model selection procedures did not find these covariates to be as effective at predicting bobcat $\psi$ in West Virginia as those associated with human impacts. However, this is most likely due to the lack of fine-scale data on bobcat prey availability on the scale of the entire state, rather than lack of support for this 
hypothesis. DST_SR is positively correlated with $\psi$, which means that bobcat $\psi$ increases at greater distances from secondary roads. As described previously, bobcats appear to be selecting for locations that have some form of minimal human impact and environmental manipulation to increase prey diversity; however, they selectively avoid areas of substantial disturbance. In contrast to the other covariates, PER_HIDEV (percentage of high intensity development) and DST_PR (distance to primary road) are minor predictors of bobcat $\psi$ and only appear in a few of the selected models. Bobcats were found to be less likely to occupy areas of intense human development (PER_HIDEV), but there was a negative relationship between $\psi$ and DST_PR, meaning that bobcats were more likely to occupy areas closer to primary roads than those that were not. Both of these relationships seem to support the hypothesis introduced previously in that bobcats selectively occupy areas with some human disturbance over areas of pure forest.

In contrast to the large number of models selected for $\psi$ for the 2015 dataset, only five were selected for inclusion in the 2015-16 dataset. However, the selected models shared the same covariates as the 2015 dataset, indicating that for 2016, similar trends were observed for bobcat $\psi$, as was documented in 2015. The most important variables in predicting bobcat $\psi$ for the 2015-2016 dataset were again ELEV and PER_AF, with the same exact trends as the 2015 data. Just as in the 2015 dataset, bobcat $\psi$ was positively associated with areas of higher elevations, presumably due to the lessened human impact; however, bobcats were again negatively associated with the percentage of total forest. Both DST_PR and ROAD_DEN appear to be moderate factors describing bobcat $\psi$ for this combined dataset, with a negative bobcat association with areas of increased road density and a decrease in $\psi$ in locations far away from primary roads. Bobcats appear to be selecting locations near primary roads so there is some form of development but avoiding areas where road density is high. However, many primary interstate 
highways also pass through relatively undeveloped or remote locations in West Virginia, so this may be the cause of some error for this predictor. DST_UC, DST_SR, and PER_HIDEV, while included in final models, did not have effects on bobcat $\psi$ that were deemed significant once their standard errors were taken into account. In conclusion, the factors having the greatest impact on bobcat $\psi$ are the same in both the 2015 and the 2015-16 datasets, adding to the weight of evidence for this hypothesis of bobcats selectively occupying habitats of minor human disturbance, rather than those located primarily in wooded areas.

\section{Acknowledgements}

We thank the many landowners of West Virginia for allowing us to sample their property for bobcats, and the many members of the West Virginia Division of Natural Resources that worked on this project and made such a large-scale data collection effort possible—especially Steve Rauch, Rob Silvester, Colin Carpenter, Eric Richmond, and Jeff McCrady. We also thank Avery Owers, Brent Mays, Jarrod Ball, Brittany Demski, Lauren Schumacher, Brittney Watson, Peter Ries, Steven Wilson, Matt Stevens, Shelby Sherman, Kyle Matt, Sheila Harris, Darren Wood, and Paige Pritt of West Virginia University for their assistance in collecting hair samples from gun brushes and processing them in the laboratory. Financial support for this dissertation was provided by the West Virginia Division of Natural Resources through a grant with the U.S. Fish and Wildlife Service Wildlife and Sport Fish Restoration Program (W-48-R) and matching state funds. 


\section{References}

Burnham, K. P., and D. R. Anderson. 2002. Model Selection and Multimodel Inference: A Practical Information Theoretic Approach. Springer-Verlag, New York, New York, USA.

Broman, D. J. A., J. A. Litvaitis, M. Ellingwood, P. Tate, and G. C. Reed. 2014. Modeling Bobcat Lynx rufus Habitat Associations Using Telemetry Locations and Citizen-Scientist Observations: Are the Results Comparable? Wildlife Biology 20:229-237.

Croteau, E. K., E. J. Heist, and C. K. Nielsen. 2010. Fine-scale population structure and sexbiased dispersal in bobcats (Lynx rufus) from southern Illinois. Canadian Journal of Zoology 88:536-545.

Chamberlain, M. J., B. D. Leopold, L. W. Burger, B. W. Plowman, and L. M. Conner. 1999. Survival and Cause-Specific Mortality of Adult Bobcats in Central Mississippi. The Journal of Wildlife Management 63:613.

Chamberlain, M. J., B. D. Leopold, and L. M. Conner. 2003. Space Use, Movements and Habitat Selection of Adult Bobcats (Lynx rufus) in Central Mississippi. The American Midland Naturalist 149:395-405.

Clare, J. D. J., E. M. Anderson, and D. M. MacFarland. 2015. Predicting Bobcat Abundance at a Landscape Scale and Evaluating Occupancy as a Density Index in Central Wisconsin. The Journal of Wildlife Management 79:469-480.

Erb, P. L., W. J. McShea, and R. P. Guralnick. 2012. Anthropogenic Influences on Macro-Level Mammal Occupancy in the Appalachian Trail Corridor. PLOS ONE 7:e42574.

Knecht, L., T.F. Rounsville Jr., and J.E. Huffman. 2015. Dynamic Hair Identification Key Ver. 1.0B. http://www.hairdatabase.com.

Hines J.E. (2006) PRESENCE-Software to estimate patch occupancy and related parameters. Available: http://www.mbr-pwrc.usgs.gov/software/presence.html.

Homer, C.G., Dewitz, J.A., Yang, L., Jin, S., Danielson, P., Xian, G., Coulston, J., Herold, N.D., Wickham, J.D., and Megown, K., 2015, Completion of the 2011 National Land Cover Database for the conterminous United States-Representing a decade of land cover change information. Photogrammetric Engineering and Remote Sensing. 81(5):345-354.

Kendall, K. C., K. S. McKelvey, et. al. 2008. Hair collection. Noninvasive Survey Methods for Carnivores. Island Press, Washington, DC 141-182.

Kitchings, J. T., and J. D. Story. 1984. Movements and Dispersal of Bobcats in East Tennessee. The Journal of Wildlife Management 48:957. 
Lesmeister, D. B., C. K. Nielsen, E. M. Schauber, and E. C. Hellgren. 2015. Spatial and Temporal Structure of a Mesocarnivore Guild in Midwestern North America. Wildlife Monographs 191:1-61.

Lewis, J. S., K. A. Logan, M. W. Alldredge, L. L. Bailey, S. VandeWoude, and K. R. Crooks. 2015. The Effects of Urbanization on Population Density, Occupancy, and Detection Probability of Wild Felids. Ecological Applications 25:1880-1895.

Litvaitis, J. A., J. A. Sherburne, and J. A. Bissonette. 1986. Bobcat Habitat Use and Home Range Size in Relation to Prey Density. The Journal of Wildlife Management 50:110.

Lombardi, J. V., C. E. Comer, D. G. Scognamillo, and W. C. Conway. 2017. Coyote, Fox, and Bobcat Response to Anthropogenic and Natural Landscape Features in a Small Urban Area. Urban Ecosystems 20:1239-1248.

Long, R. A., T. M. Donovan, P. MacKay, W. J. Zielinski, and J. S. Buzas. 2011. Predicting Carnivore Occurrence with Noninvasive Surveys and Occupancy Modeling. Landscape Ecology 26:327-340.

MacKenzie D., and Hines J.E. (2014). PPRESENCE: R Interface for the Program PRESENCE. http://www.mbr-pwrc.usgs.gov/software/presence.html.

MacKenzie, D. I., J. D. Nichols, G. B. Lachman, S. Droege, J. A. Royle, and C. A. Langtimm. 2002. Estimating Site Occupancy Rates When Detection Probabilities Are Less Than One. Ecology 83:2248-2255.

Mackenzie, D. I., and J. A. Royle. 2005. Designing Occupancy Studies: General Advice and Allocating Survey Effort. Journal of Applied Ecology 42:1105-1114.

Maxwell, A. E., M. Strager, C. Yuill, E. Austin, and W. Kordek. 2012. Creation of High Resolution Land Cover and Forest Cover for West Virginia Relative to 2011 NAIP Orthophotography. Proceedings of the 2012 West Virginia GIS Conference, West Virginia GIS Technical Center.

McCord, C. M. 1974. Selection of Winter Habitat by Bobcats (Lynx rufus) on the Quabbin Reservation, Massachusetts. Journal of Mammalogy 55:428.

McCord, C.M. and J.E. Cardoza. 1982. Bobcat and Lynx. Pages 728-766 in J.A. Chapman and G.A. Feldhammer, eds. Wild mammals of North America: biology, management, and economics. Johns Hopkins Univ. Press, Baltimore, Md.

McGarigal, K., S. A. Cushman, and E. Ene. 2012. FRAGSTATS v4: Spatial Pattern Analysis Program for Categorical and Continuous Maps. Computer software program produced by the authors at the University of Massachusetts, Amherst. URL: http://www.umass.edu/landeco/research/fragstats/fragstats.html 
Progulske, D.R. 1952. The Bobcat and its Relation to Prey Species in Virginia. M.S. Thesis. Virginia Poly. Inst., Blacksburg, Va. 135 pp.

$R$ Core Team (2012). R: A Language and Environment for Statistical Computing. $R$ Foundation for Statistical Computing, Vienna, Austria. ISBN 3-900051-07-0, URL: http://www.Rproject.org/

Randall, S. M., M. D. Grant, and F. W. Brian. 2016. Forests of West Virginia, 2016. Resource Update FS-123. Newton Square, PA; U.S. 4.

Uhlig, H. G., and H. L. Wilson. 1952. A Method of Evaluating an Annual Mast Index. The Journal of Wildlife Management 16:338-343.

United States Census Bureau. 2017. TIGER/Line Shapefiles (2016). URL: https://www.census.gov/geo/maps-data/data/tiger-line.hmtl.

Wang, Y., M. L. Allen, and C. C. Wilmers. 2015. Mesopredator Spatial and Temporal Responses to Large Predators and Human Development in the Santa Cruz Mountains of California. Biological Conservation 190:23-33.

White, G. C. 1982. Capture-Recapture and Removal Methods for Sampling Closed Populations. Los Alamos National Laboratory. 


\title{
Chapter 4 - Estimating the Size of West Virginia's Bobcat Population
}

\begin{abstract}
Sustainable management of harvested wildlife species can be difficult without reliable estimates of the population densities. Bobcats (Lynx rufus) are medium-sized felids of conservation concern to states in which these animals are harvested since they are included under the Convention on International Trade in Endangered Species of Wild Fauna and Flora (CITES) Appendix II. The objective of this research was to use the newly developed bobcat hair snare cubby to estimate abundance and density of bobcats in West Virginia. Between the 2015 and 2016 field seasons a total of 1,500 different $10 \mathrm{~km}^{2}$ locations were sampled for bobcats. A total of 378 bobcat detections were recorded, and of these, $60.8 \%(n=230)$ were successfully genotyped. These detections originated from 212 individual bobcats that were captured once, and 9 individuals that were captured twice. However, this quantity of recaptures was not sufficient for use in spatially explicit capture-recapture (SECR) so a combination of minimum known alive (MKA) and a Royle-Nichols model were used to estimate relative bobcat density between ecological regions and abundance, respectively. The Royle-Nichols model estimated abundance was $10,926.51 \pm 2,960.43$, with a $95 \%$ confidence interval of $6,018.11-19,838.26$. Based on the lowest estimated population size, as calculated by the standard error of the Royle-Nichols model ( $n=7,966$ ), and a post-harvest population, an average harvest of roughly 1,700 individuals per year should result in a stable or growing bobcat population. Finally, the current management model used for projecting population change was validated empirically and should be updated with more current demographic data.
\end{abstract}




\section{Introduction}

The bobcat is a medium sized member of the Felidae family that is native to North America. Bobcats are found throughout the United States, except for Delaware (Roberts and Crimmins 2010), and in many of the states in which they reside, bobcats are hunted and trapped, mainly for their fur. However, bobcats remain a conservation concern for states that allow regulated hunting and trapping due to their inclusion in the Convention on International Trade in Endangered Species of Wild Fauna and Flora (CITES) Appendix II in 1977, since they are a look-alike species to the endangered Iberian lynx (Lynx pardinus) (CITES 17 ${ }^{\text {th }}$ Meeting 2016). Compliance with CITES regulations requires state wildlife agencies to report that all harvests are sustainable and will not have long-term detrimental impacts on the population in question in addition to any changes in bobcat hunting and trapping regulations. To ensure that these obligations under CITES are met, state wildlife agencies require demographic information and population estimates, to make informed management decisions.

\section{Spatially Explicit Capture-Recapture}

Spatially explicit capture-recapture (SECR) (Borchers and Efford 2008) is a relatively new type of modeling that combines the population estimation capabilities of a capture-recapture model with parameters that are accounted for in physical space. One of the major drawbacks of traditional capture-recapture modeling was that study sites located near the edge of the sampling area had increased error since spatial processes off the sampling grid were ignored (Royle et al. 2013). Traditional methods also did not account for the fact that proximity of the detector to an animal's home range significantly impacted detection probabilities (Borchers and Efford 2008).

SECR models evaluate the relative position of individual animal home ranges on a sampling grid 
based on spatial recaptures, or recaptures that occur at different sites. However, without these spatial recaptures, the method is unable to provide any density estimation. SECR models have already been applied to estimate bobcat density in Texas (Thornton and Pekins 2015), central Wisconsin (Clare et al. 2015), and the Appalachian Mountains of Virginia, directly on the border with West Virginia (Morin et al. 2018). While these studies used SECR to estimate bobcat abundance, none used hair snares for bobcat detection. SECR models are more powerful than traditional capture-recapture methods, but they still require many recapture events, which may be difficult to achieve using hair-snares.

\section{Royle-Nichols Model}

The Royle-Nichols modeling framework uses repeated detection-nondetection data to arrive at an estimate of abundance (Royle and Nichols 2003). This model is based on the premise that detection probability varies due to abundance of the species of interest at each site. The pattern of repeated detections is then used to fit estimated abundances to a probability distribution, with a Poisson distribution being the most common. While this model is advantageous in that it does not require capture-recapture data to estimate abundances, the three important assumptions of this model can limit its utility: 1) detections must be independent, 2) the number of animals at a site cannot vary between survey periods (assumed population closure), and 3) the detection probability of each animal is constant over time. These requirements limit the use of this type of model in estimating animal abundances; however, a recent capture-recapture study of the fisher (Martes pennanti) found that density estimates were nearly identical between the Royle-Nichols and a SECR model when the size of the sampling 
unit was roughly equivalent to, or slightly smaller than, average home range size (Linden et al. 2017).

West Virginia Bobcat Management Model

The current method used in West Virginia to determine the yearly change in population size is based on Crowe's (1975) model, originally designed for a harvested bobcat population in Wyoming. Currently, the WVDNR uses this model with bobcat demographic data collected in the late 1970s and early 1980s (Fox and Fox 1982). The yearly change in population size is predicted by Crowe's model $\mathrm{Y}=\mathrm{S}_{\mathrm{a}}+\mathrm{F}\left(\mathrm{S}_{\mathrm{y}}\right)$, where $\mathrm{Y}$ is the yearly change in population size, $\mathrm{S}_{\mathrm{a}}$ is the adult survival, $\mathrm{F}$ is the number of female young produced per female, and $\mathrm{S}_{\mathrm{y}}$ is juvenile survival. Based on this model and the data collected in 1982, as well as in other localities that manage bobcats, $21 \%$ of the total yearly surplus can be harvested, while still leaving sufficient numbers for sustainable population growth (personnel communication: Rich Rogers, WVDNR). However, these demographic data collected in 1982 may no longer be reflective of the current West Virginia bobcat population. Importantly, another demographic and health study was recently completed on West Virginia’s bobcats (Landry 2017), and these data are available for use in modeling.

\section{Study Objectives}

The density of bobcats, as derived from nonharvest data, in West Virginia has never been evaluated in a formal study. To assist the WVDNR in ensuring continued, sustainable bobcat harvests, abundance and density estimates for the bobcat population in West Virginia were generated using a combination of spatially-explicit capture-recapture and occupancy modeling. 
The following were the objectives of this research project: 1) Estimate the abundance and density of bobcats across the study area in West Virginia, as well as each ecological region (Uhlig and Wilson 1952, appendix Figure 1); 2) compare abundance and density estimates between occupancy Royle-Nichols models (Royle and Nichols 2003) and SECR; 3) compare and contrast the estimated population abundances and densities to those calculated using West Virginia's current bobcat population model; and 4) determine if a new population growth model would better represent West Virginia’s bobcat population than what is currently used for management.

\section{Materials and Methods}

\section{Field Methods}

Both the bobcat presence-absence and individual identity data for this project originated from a statewide hair snare survey. The hair snare used for this study was developed specifically for the West Virginia Division of Natural Resources Bobcat Project (see Chapter 2). It was constructed of thin, blue corrugated plastic and was erected like a tent tall enough that an adult bobcat could move directly through the device without crouching. Four 0.30-caliber gun brushes were affixed to each entranceway of the cubby to forcibly remove hair from any animals entering the device. A carpet pad affixed to the inside of the device was coated with two scent lures: a mixture of beaver (Castor canadensis) castoreum and artificial catnip oil and a separate Light Skunk paste calling lure (Cage Magic Lures, Barstow, CA). In addition to the scent lures, a small food bait of Caven’s Minnesota Brand Bobcat Chunk Bait (Minnesota Trapline Products, Pennock, MN) was placed in a mock cache hole that was dug directly under the center of the erected cubby. 
Sampling locations were selected after using an artificial grid latticework of $10 \mathrm{~km}^{2}$ cells to divide West Virginia into discrete sampling units. This cell size was specifically selected based on the recommendations of White (1982), that for capture-recapture studies, at least two sampling devices should be placed in the smallest possible home range. Female bobcats generally have smaller home ranges than males (Lovallo and Anderson 1996), and their average home range size in West Virginia was thought to be $\sim 20 \mathrm{~km}^{2}$, based on work completed in similar habitats of neighboring Virginia (Progulske 1952, McCord and Cordoza 1982). A total of 60 clumped groupings of 25 cells $\left(5 \times 5,250 \mathrm{~km}^{2}\right)$ were surveyed, with 30 surveyed in 2015 and the second 30 in 2016. However, nine locations sampled in 2015 were resampled in 2016 as a direct comparison of sampling performance between seasons. Each clumped survey site $\left(250 \mathrm{~km}^{2}\right)$ was selected based on numerous criteria, with the most important being an attempt to evenly minimize distances between trapping grids and maximize the accuracy of the final interpolated population estimate. Sampling sites were also purposefully selected to include both suspected high quality and marginal bobcat habitat, as well as locations with highly varied bobcat densities. Within each $10 \mathrm{~km}^{2}$ cell selected for study, a single hair snare cubby was placed opportunistically based on available habitat types and landowner permissions. When available, cubbies were selectively placed on or adjacent to game trails or dirt roads in forested habitats, as recommended by Clare et al. (2015) for increasing the probability of detecting bobcats. Cubbies were also selectively placed near natural funnels such as rock formations, when available. To collect data on bobcat habitat selection, many cubbies were deployed either deliberately, or due to extraneous circumstances, in what was suspected to be suboptimal bobcat habitat. Each site was sampled for a four-week period referred to henceforth as a session, which was further broken down into four 1-week samplings. At the end of every week, the gun brushes from each 
cubby were collected, regardless of whether a hair sample was visible. The scent lures and bait were also refreshed every week when the gun brushes were replaced. Between the second and third weeks of sampling, the cubbies were moved roughly $15 \mathrm{~m}$ from the original location, to reduce a potential trap-avoidance response (Kendall et al. 2008). During each field season a total of five trapping sessions were held. Each session was comprised of four weeks of continuous sampling at the same location. The sessions took place between March-August in 2015 and March — July in 2016, with six different 25-cell grids being deployed statewide at any given time (Table 1). At the conclusion of a session, each cubby was removed and placed in a new sampling location.

\section{Laboratory and Molecular Methods}

Gun brushes collected in the field were examined for the presence of hair samples under a dissecting microscope. Entire hairs were removed from each of the four gun brushes collected from a single cubby and were placed into a single $1.5 \mathrm{~mL}$ microcentrifuge tube containing $100-$ $125 \mu \mathrm{L}$ of molecular grade distilled water. Collected hair samples were stored at $-20^{\circ} \mathrm{C}$ until DNA extraction with a Qiagen (Bethesda, MD) DNeasy 96 Blood \& Tissue kit using a modified protocol. To completely digest the entire hair samples, $20 \mu \mathrm{L}$ of proteinase $\mathrm{K}(20 \mathrm{mg} / \mathrm{mL}), 30 \mu \mathrm{L}$ of 1M DTT (dithiothreitol), and 250 $\mu \mathrm{L}$ of buffer ATL were added. The samples were then incubated at $56^{\circ} \mathrm{C}$ until completely dissolved, which occurred within $5-10$ hours. The additional DNA extraction procedures were completed following the manufacturer's recommendation except that the final elution step was modified to consist of two $100 \mu \mathrm{L}$ buffer AE elutions. This second elution was used to ensure the maximum possible recovery of DNA from the filter of the spin column. Following elution, sample plates were dried on a vacuum 
centrifuge at $60^{\circ} \mathrm{C}$ for six hours until roughly $75 \mu \mathrm{L}$ of buffer $\mathrm{AE}$ remained in each well. Due to budgetary constraints, only samples that were suspected to be of felid origin by microscopy (Hairdatabase.com 2015), those containing five or more hairs, or samples of less than five hairs with entire follicles were extracted.

Species identification of collected hair samples was completed using the quantitative polymerase chain reaction (qPCR). Two new primer pairs were specifically developed for this study to allow for the reliable qPCR species identification from degraded or fragmented hair samples that might otherwise fail to be Sanger sequenced, yet still have enough DNA for qPCR speciation (see Chapter 2). The first primer pair, FelidV was used to determine which samples were of felid origin, and the second primer pair LynX, only amplifies the mitochondrial DNA (mtDNA) of the bobcat and Canada lynx (Lynx canadensis). To identify species, a two-step PCR procedure was used: an initial qPCR with FelidV identified which samples were of felid origin and then a separate qPCR with primer set LynX was used to determine which felid samples originated from a bobcat.

Positive and negative controls were run with each qPCR reaction, and were used to establish a CQ cutoff for a positive result. After multiple runs, it was determined that a control sample of two bobcat hairs (the minimum considered to be needed to produce a usable sample), which became the positive cutoff, would reliably have a CQ of roughly 33.5 for the FelidV primer set and a CQ of roughly 32.5 for the LynX primer set. As such, samples producing a CQ value greater than these critical thresholds were considered positive for that particular reaction. Samples producing a positive result for the $\mathrm{V}$ primer set, but a negative result for $\mathrm{X}$ were assumed to have originated from domestic cats (Felis catus), since this is the only other felid species known to inhabit the sampling areas. Canada lynx are not known to inhabit West 
Virginia, so a positive result for qPCR using primer set LynX was recorded as a bobcat detection. Universal mtDNA controls were not used to verify PCR functionality.

Samples positively determined to have originated from a bobcat were preliminarily screened with a three-locus multiplex of highly polymorphic microsatellites (FCA008, FCA90, and FCA77) (Menotti-Raymond and O’Brien 1995, Menotti-Raymond et al. 1997, 1999). Based on population genetics research on West Virginia's bobcat population (Chapter 5) it was determined that the non-exclusion probability of identity of these three loci was $2.279 \times 10^{-4}$, with a $p$-sib of 0.047 . The total reaction volume for each multiplex PCR was $10 \mu \mathrm{L}$ and consisted of $5 \mu \mathrm{L}$ of the 2x Qiagen Multiplexing Master Mix (Germantown, MD), 300nmol of each individual primer, and at least 10ng of DNA template. PCR reaction conditions were as follows: 15-minute hot-start at $95^{\circ} \mathrm{C}, 45$ cycles of $94^{\circ} \mathrm{C}$ for 30 s, $57^{\circ} \mathrm{C}$ for 90 s, and $72^{\circ} \mathrm{C}$ for 90 s, with a final extension of $72^{\circ} \mathrm{C}$ for 10 minutes. Amplified DNA fragments were resolved on a BeckmanCoulter GeXP Genetic Analysis System (AB SCIEX, Washington, D.C.) and fragment sizes were recorded using the associated GeXP fragment analysis software.

After two initial PCRs, samples failing to produce genotypes for at least two of the three loci were removed from the project. Additional PCRs were completed on the included samples such that each allele call needed to be seen in at least two consecutive PCRs before it was recorded as part of a final genotype for a sample. Some samples were amplified four or more times in this manner to produce replicates of the same genotypes. The program GENECAP v1-2 (Wilberg and Dreher 2004) was then used to find samples with matching genotypes. These were then amplified using the additional microsatellite loci of multiplexes A (FCA23, FCA25) and C (FCA43, 6HDZ700, and FCA45) (Menotti-Raymond and O’Brien 1995, Menotti-Raymond et al. 1997, 1999, Williamson et al. 2002). Once a combination of loci had been amplified for each 
sample pair where $p$-sib $<10^{-3}$, samples found to still be matching at all loci were then considered to be recaptures. Samples failing to provide a complete match, or a mismatch greater than one allele, were considered to have been derived from separate individuals.

\section{Minimum Known Alive}

Relative minimum densities were calculated for each ecological region based on the raw number of bobcat genotypes detected in each location, which is known as the minimum known alive (MKA). MKA is a calculation based on the number of unique animals captured on the landscape during the survey, and is useful for direct comparisons between sampled locations. It is not a measurement of the true population abundance or density, since it is much lower than what is actually expected to be on the landscape. The relative minimum densities were calculated by dividing the number of unique bobcat detections in a particular ecological region by the total area surveyed in that same region. This same procedure was then completed for the number of detections when taking into account the mean detection probability of each survey grid, as calculated in Chapter 3.

\section{Royle-Nichols Model}

The program PRESENCE v.12.6 (Hines 2006) was used to fit a Royle-Nichols model based on the collected bobcat presence-absence data. However, the entire dataset was not used in order to reduce the amount of error in model construction. Since final abundance estimates are contingent on capture probabilities of each individual staying the same over time, the nine sites that were sampled in both 2015 and 2016 were removed, leaving a total of 51 survey grids in the remaining dataset. Due to the previous occupancy modeling work completed (Dissertation 
Chapter 3), the best models for detection ( $p$ ) and occupancy ( $\psi$ ) for the 2015 and the 2015 2016 datasets, both important parameters for the Royle-Nichols model, were already known (Tables 8, 10, 12). However, since this dataset is not entirely the same as the others, both the best 2015 and the 2015 - 2016 models were run to determine which model had the most support. The best model was then selected based on $\triangle \mathrm{AIC}=0$. PRESENCE was then used to run a goodness of fit test on the selected model with a null hypothesis that the model adequately fit the data ( $\alpha=$ $0.05)$. 
Table 12 - Covariates used to model probability of detection $(p)$ and occupancy probability $(\psi)$ in bobcats of West Virginia. The predictor type is the parameter estimates for which that covariate was used. The expected result is the direction of the correlative relationship between each coavariate and bobcat $\mathrm{p}, \psi$, or both. Hypotheses associated with conclusions reached from other studies are marked with a superscript.

\begin{tabular}{|c|c|c|c|}
\hline Covariate & Description & $\begin{array}{l}\text { Predictor } \\
\text { Type }\end{array}$ & $\begin{array}{l}\text { Expected } \\
\text { Result }\end{array}$ \\
\hline DST_MR & Linear distance (m) to nearest minor paved road. & $p, \psi$ & $+1,2$ \\
\hline DST_PR & $\begin{array}{l}\text { Linear distance (m) to nearest interstate highway. These roads are travelled } \\
\text { by large volumes of traffic in certain areas but also pass through otherwise } \\
\text { remote locations. }\end{array}$ & $p, \psi$ & $+1,2$ \\
\hline DST_SR & Linear distance (m) to nearest secondary road, such as state routes. & $p, \psi$ & $+1,2$ \\
\hline DST_UC & $\begin{array}{l}\text { Linear distance }(m) \text { to nearest urban cluster, or locations with populations } \\
>2,500 \text {, as defined by the U.S. Census Bureau for } 2015 \text {. }\end{array}$ & $p, \psi$ & $++^{2}$ \\
\hline ELEV & Elevation (m) of hair snare cubby location. & $\psi$ & + \\
\hline PER_AF & $\begin{array}{l}\text { The percentage of landscape that is covered with any type of forest within } \\
\text { a radius of } 2,500 \mathrm{~m} \text { of the cubby hair snare location. }\end{array}$ & $\psi$ & $+1,2,3$ \\
\hline PER_HIDEV & $\begin{array}{l}\text { Buffer calculated percentage of high intensity human development. This } \\
\text { class is reserved specifically for urban centers or areas of intense human } \\
\text { usage. }\end{array}$ & $\psi$ & $-2,4,6$ \\
\hline ROAD_DEN & $\begin{array}{l}\text { Road density }\left(\mathrm{km} / \mathrm{km}^{2}\right) \text { of roads as calculated around pixel-meter }(30 \times 30) \\
\text { raster cell at a radius of 2,500m from the U.S. Census Bureau } 2015 \text { TIGER } \\
\text { roads dataset. }\end{array}$ & $p, \psi$ & $-2,6$ \\
\hline SESSION & $\begin{array}{l}\text { A categorical variable with } 5 \text { classes that correspond to the } 4 \text {-week period, } \\
\text { during which, each location was sampled. More information on the session } \\
\text { dates can be found in dissertation chapter } 2 \text {. Session } 1 \text { was encoded as zero } \\
\text { to be compared with } 2,3,4 \text {, and } 5 \text {. }\end{array}$ & $p$ & - \\
\hline YEAR & $\begin{array}{l}\text { A categorical variable of whether a particular site was sampled in } 2015 \text { (0) } \\
\text { or } 2016 \text { (1). }\end{array}$ & $p$ & 0 \\
\hline
\end{tabular}

${ }^{1}$ Clare et al. (2015), ${ }^{2}$ Lesmeister et al. (2015), ${ }^{3}$ Erb et al. (2012), ${ }^{4}$ Wang et al. (2015)

${ }^{5}$ McCord (1974), Litvaitis et al. (1986), Chamberlain et al. (1999, 2003), Long et al. (2011), Lesmeister et al. (2015), ${ }^{6}$ Lombardi et al. (2017).

\section{Results}

Of the 2,014 hair samples collected in 2015, 1,641 were tested via qPCR, and 278 (13.8\%) were found to have originated from a bobcat. In 2016, a total of 2,108 hair samples were collected, 1,301 were tested via qPCR, of which 100 (7.7\%) were of bobcat origin. For both 
2015 and 2016, a total of 21,000 trap nights of surveys were completed, since 30 grids of 25 cells were sampled four weeks each year. For 2015 an average bobcat detection rate of 1.3 bobcats per 100 trap nights was observed and for 2016 it was 0.47 bobcats per 100 trap nights. However, sites of marginal bobcat habitat were selected and sampled to gather covariates for this dataset, so the trap efficiency of the bobcat hair snare cubby in ideal bobcat habitat is probably much higher. Over the course of the two-year study a total of 1,275 unique sites were examined for bobcat presence or absence, 225 were visited twice, and a total of 378 bobcat detection events were recorded, for an overall bobcat detection rate of 0.9 detections per 100 trap nights (Figures $9,10)$. 


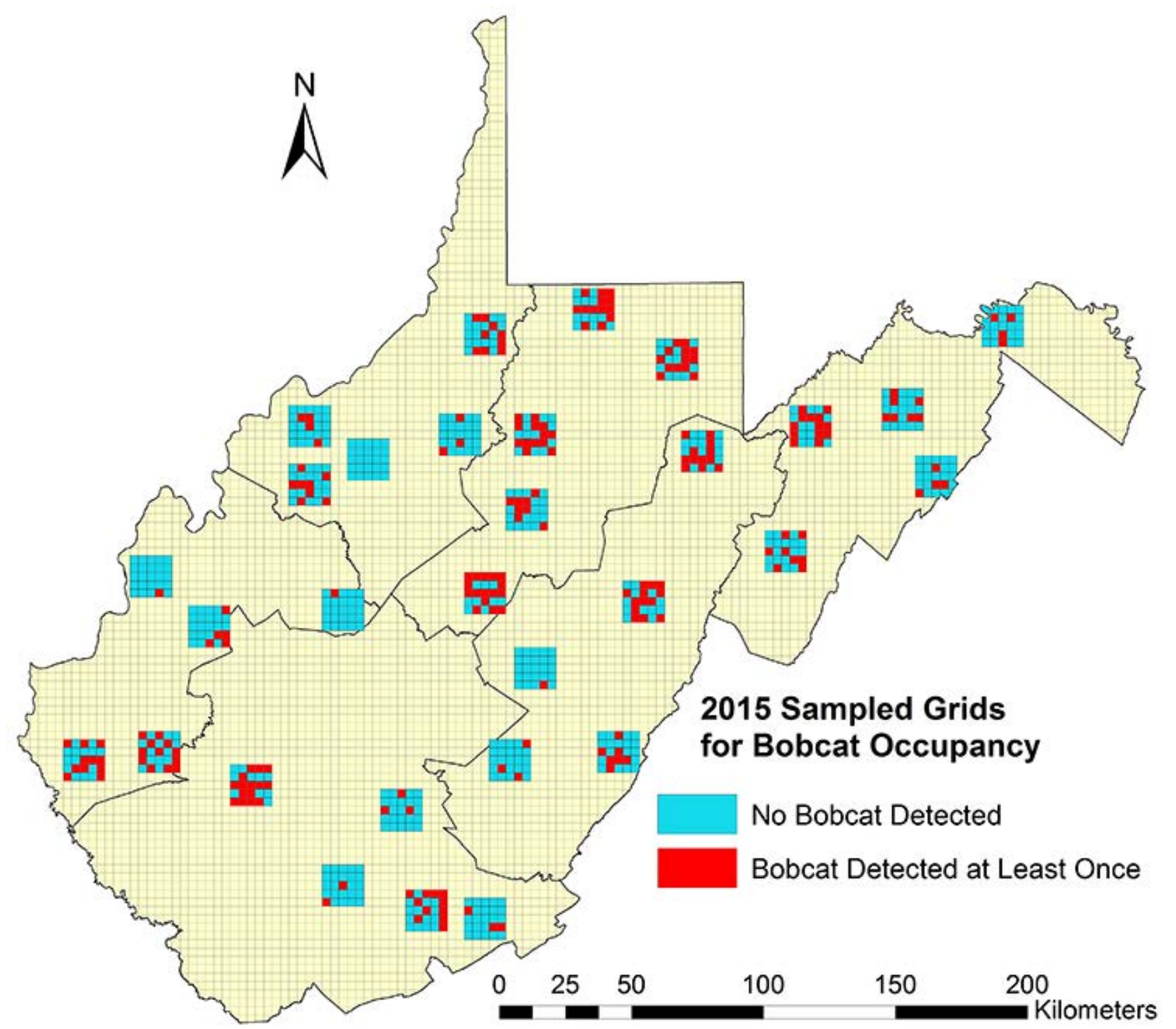

Figure 9 - Map of the locations surveyed for bobcats in West Virginia between March - August of 2015. Each $5 \times 5$ set of cells was sampled for a total of four weeks, with one bobcat hair snare cubby being placed in each cell. Cells marked with blue on the map are locations that were sampled but a bobcat was not detected at those locations. Cells marked in red indicate that at least one bobcat detection was recorded at that site. Background cells in tan were not sampled. The black lines on the map separate West Virginia into its separate ecological regions (appendix Figure 1). 


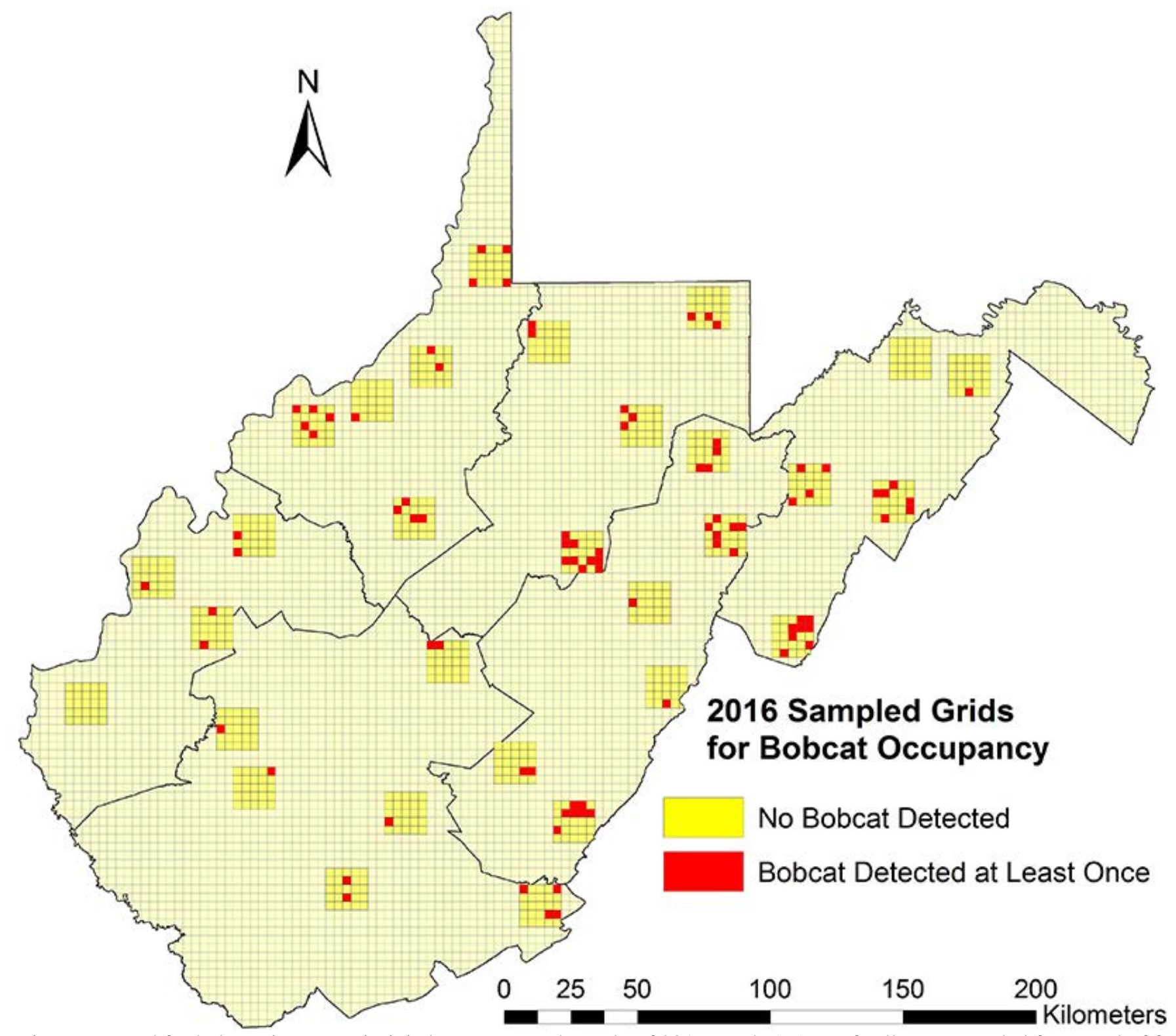

Figure 10 - Map of the locations surveyed for bobcats in West Virginia between March - July of 2016. Each $5 x 5$ set of cells was sampled for a total of four weeks, with one bobcat hair snare cubby being placed in each cell. Cells marked with blue on the map are locations that were sampled but a bobcat was not detected at those locations. Cells marked in red indicate that at least one bobcat detection was recorded at that site. Background cells in tan were not sampled. The black lines on the map separate West Virginia into its separate ecological regions (appendix Figure 1). 
From the 378 samples that originated from bobcats, a total of 230 (60.8\%) were successfully genotyped for at least two of the three loci of the initial sample screening multiplex (FCA008, FCA90, and FCA77). Only a small number of samples ( 3\%) appeared to have been collected from mixed felid species, or multiple bobcat individuals, and were removed from the study. Duplicate genotypes were found for 18 samples with a $p$-sib $<0.001$, indicating that these samples were recaptures. Of the 230 samples, 212 bobcats were captured once and 9 bobcats were captured twice. No bobcat was recaptured at the same site where it was originally captured. Also, 55.6\% of all recaptures occurred during the same sampling week that an individual had already been captured at a nearby site. Most (55.6\%) recaptures occurred a distance of three or less sampling cells from the original capture location. Due to the insufficient number of spatial recaptures recorded during different sampling weeks, spatially-explicit capture-recapture models could not be run on the dataset. Instead, comparisons of relative abundance and density between sampled sites were made using Minimum Known Alive (MKA), since individual identification information for each bobcat was available.

\section{Minimum Known Alive}

Of the 221 bobcat individuals identified during this hair snare survey, the distribution of individuals between sampling sites was not even. Eight sites did not produce any successful bobcat genotypes, and in contrast, 21 individual bobcats were captured during the 4-week 2015 study of Braxton County in ecoregion 4, with no recaptures (appendix Table 1). The minimum bobcat density of this study site, post-harvest, was calculated as 8.40 individuals / $100 \mathrm{~km}^{2}$. If different animals did not have overlapping home ranges, the average home range at this site was estimated at $11.90 \mathrm{~km}^{2}$ per bobcat, roughly $59.5 \%$ of the predicted $20 \mathrm{~km}^{2}$ average bobcat home 
range size. However, the density calculated for this site is the minimum value, and does not take into account the imperfect detection of the hair snare cubby. Using the 4-week average detection probability for this particular site (Dissertation Chapter 3) of $0.567 \pm 0.066$, the minimum density, when adjusted for detection probability is estimated at $15.18 \pm 1.001$ individuals / $100 \mathrm{~km}^{2}$. To accommodate this many individuals within the $250 \mathrm{~km}^{2}$ study grid, the average nonoverlapping home range size would have to be reduced to roughly $6.75 \mathrm{~km}^{2}$, or only $33.8 \%$ of the predicted $20 \mathrm{~km}^{2}$. While this site had the greatest minimum density, it was not the only one with a fairly large calculated minimum density. At the 2015 site in Boone County of ecoregion 3, a minimum of 16 unique individuals ( 6.4 bobcats / 100 $\mathrm{km}^{2}$ ) was detected, with no recaptures. Surprisingly, this site was revisited in 2016, and only two unique individuals were detected (with one recapture). Neither of these 2016 captured samples had genotype profiles matching the 16 individual animals identified at this location in 2015.

When examined ecoregionally, the estimated minimum density ranged from $1.09-2.76$ bobcat individuals / $100 \mathrm{~km}^{2}$ (Table 13). However, these estimates did not take into account the imperfect detection of the hair snare cubby. Using the 4-week average capture probability of a bobcat (Dissertation Chapter 3) as calculated for the combined 2015 - 2016 dataset and each ecoregion as a correction factor, the estimated minimum density ranged from $2.87 \pm 0.072-6.90$ \pm 0.108 individuals $/ 100 \mathrm{~km}^{2}$, with the greatest minimum density being located in ecoregion 4 , or north-central West Virginia (Table 13). 
Table 13 - The number of sites sampled, number of bobcat detections, number of unique individuals sampled, minimum bobcat density, mean ecoregion (appendix Figure 1) detection probability and minimum bobcat density averaged across each ecoregion when taking detection probability into account for bobcat hair samples collected in West Virginia between 2015 - 2016. Minimum Known Alive (MKA) is not a measurement of actual census population size or density.

\begin{tabular}{|c|c|c|c|c|c|c|}
\hline Ecological Region & 1 & 2 & 3 & 4 & 5 & 6 \\
\hline Sites Sampled $\left(250 \mathrm{~km}^{2}\right)$ & 10 & 11 & 12 & 9 & 10 & 8 \\
\hline Bobcat Detections & 63 & 64 & 62 & 98 & 50 & 41 \\
\hline Minimum Known Alive (MKA) & 34 & 30 & 36 & 62 & 28 & 22 \\
\hline MKA bobcats / 100km² & 1.36 & 1.09 & 1.20 & 2.76 & 1.12 & 1.10 \\
\hline $\begin{array}{c}\text { Mean Ecoregional Detection } \\
\text { Probability }\end{array}$ & $\begin{array}{c}0.420 \pm \\
0.066\end{array}$ & $\begin{array}{c}0.379 \pm \\
0.066\end{array}$ & $\begin{array}{c}0.374 \pm \\
0.065\end{array}$ & $\begin{array}{c}0.403 \pm \\
0.039\end{array}$ & $\begin{array}{c}0.376 \pm \\
0.034\end{array}$ & $\begin{array}{r}0.365 \pm \\
0.034\end{array}$ \\
\hline $\begin{array}{l}\text { MKA bobcats / } 100 \mathrm{~km}^{2} \text { Accounting } \\
\text { for Detection Probability }\end{array}$ & $\begin{array}{l}3.24 \pm \\
0.090\end{array}$ & $\begin{array}{l}2.87 \pm \\
0.072\end{array}$ & $\begin{array}{l}3.24 \pm \\
0.078\end{array}$ & $\begin{array}{l}6.90 \pm \\
0.108\end{array}$ & $\begin{array}{l}2.95 \pm \\
0.038\end{array}$ & $\begin{array}{l}3.06 \pm \\
0.037\end{array}$ \\
\hline
\end{tabular}

Royle-Nichols Model

The Royle-Nichols model for the 2015 - 2016 dataset was determined to be superior to the 2015 model alone (Table 14). The average value of $\lambda$ was calculated to be $1.84 \pm 0.60$, and it ranged from $1.17-3.85$. This parameter is the abundance of each study cell $\left(10 \mathrm{~km}^{2}\right)$, averaged across all sites. The combined average estimated abundance was 2,341.55 \pm 634.42 bobcats, with a range of 1,491.80 - 4,908.45 and an average 95\% confidence interval of 1,289.68 -4,251.34. The null hypothesis of the goodness of fit test of PRESENCE was not rejected $(p=0.37)$ since the model adequately fit the data. The total sampled area, which included both purported excellent and mediocre bobcat habitat, was $12,750 \mathrm{~km}^{2}$. West Virginia has roughly $59,500 \mathrm{~km}^{2}$ of bobcat habitat (personal communication, Rich Rogers, WVDNR), so extrapolating this estimate out over areas that were not sampled, the estimated bobcat population size for the entire state 
was $10,926.51 \pm 2,960.43$, with a $95 \%$ confidence interval of 6,018.11 - 19,838.26. Population estimates for each ecological region were calculated using the amount of bobcat habitat available in each ecological region as a proportion of the total RN estimated abundance. The population estimates for each ecological region were: 1) 1,611.52 \pm 436.62 , 2) $1,835.07 \pm 497.20$, 3) $3,067.97 \pm 831.24$, 4) $1,575.37 \pm 426.83$, 5) $1,502.59 \pm 407.11$, 6) 1,335.16 \pm 361.75 .

The covariates of DST_PR (distance to primary road), ELEV (elevation), and PER_AF (percent all types of forest) were found to be significant for $\lambda$ (parameter estimates did not cross 0) and YEAR and S1 - S5 were significant for estimating detection (Table 15). Bobcat abundance $(\lambda)$ was positively correlated to the distance from primary roads (DST_PR), elevation (ELEV), and negatively correlated with the total percentage of all forest types (PER_AF).

Bobcat detection was negatively associated with the 2016 dataset, indicating a significant drop in detection between years. Also, detection was negatively associated with all session variables except for S2. Finally, detection was positively correlated to the distance to minor roads with detections increasing at greater distances from minor roads. 
Table 14 - Selected models for abundance $(\lambda)$ and detection probability $(p)$ using the 2015-16 and just 2015 West Virginia bobcat presence-absence dataset. -2 Ln L is 2 times the negative log likelihood of that particular model. $\triangle \mathrm{AIC}=$ Akaike's Information Criterion. $\omega \mathrm{r}$ is the model weight ratio, recalculated for these selected models. $\mathrm{K}^{*}$ is the number of parameters (Table 12) included in the model. This number is greater than the number of parameters listed under model because SESSION has to be coded as four separate variables (SESSION 1 was reference and absorbed into the intercept and SESSIONS 2, 3, 4, and 5 were included) in RPRESENCE.

\begin{tabular}{|c|c|c|c|c|c|}
\hline Year & Model & $-2 \operatorname{Ln} L$ & $\Delta \mathrm{AIC}$ & $\omega^{\mathrm{r}}$ & $\mathrm{K}^{*}$ \\
\hline \multirow[t]{2}{*}{ 2015-2016 } & $p \sim$ DST_MR + YEAR + SESSION, & 2493.82 & 0.00 & 1.00 & 13 \\
\hline & $\begin{array}{c}\lambda \sim \text { DST_PR + ROAD_DEN }+ \text { ELEV + } \\
\text { PER_AF }\end{array}$ & & & & \\
\hline 2015 & $\begin{array}{c}p \sim \text { DST_SR + DST_MR + DST_UC + } \\
\text { SESSION } \\
\lambda \sim \text { DST_UC + PER_HIDEV + } \\
\text { ROAD_DEN + ELEV + PER_AF }\end{array}$ & 2540.51 & 49.69 & 0.00 & 14 \\
\hline
\end{tabular}

Table 15 - $\beta$ values (with standard errors) for the BEST model from the 2015 - 2016 combined dataset for probability of detection $(p)$ and occupancy $(\psi)$ of the West Virginia bobcat presence-absence and the included covariates (Table 12). This model was then used to model abundance in a Royle-Nichols framework using the program PRESENCE. Values in bold indicate that the parameter estimate, when combined with the standard error, did not cross zero, and is a significant predictor.

\begin{tabular}{|c|c|c|c|}
\hline & Model & $p$ & $\lambda$ \\
\hline \multirow{11}{*}{ 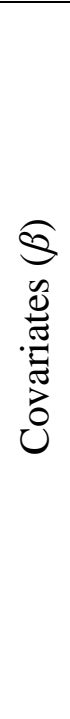 } & DST_PR & - & $0.086 \pm 0.063$ \\
\hline & ROAD_DEN & - & $0.008 \pm 0.074$ \\
\hline & ELEV & - & $0.173 \pm 0.066$ \\
\hline & PER_AF & - & $-0.128 \pm 0.057$ \\
\hline & DST_MR & $0.109 \pm 0.059$ & \\
\hline & YEAR & $-0.964 \pm 0.142$ & - \\
\hline & S1 & $-2.693 \pm 0.354$ & - \\
\hline & S2 & $0.376 \pm 0.175$ & - \\
\hline & S3 & $-0.376 \pm 0.183$ & - \\
\hline & S4 & $-0.467 \pm 0.194$ & - \\
\hline & S5 & $-0.636 \pm 0.204$ & - \\
\hline
\end{tabular}




\section{Discussion}

While a sufficient number of recaptures were not collected, precluding the use of SECR to calculate density, population estimates were still calculated using other methods. From the starting dataset of 378 bobcat detections and 221 uniquely genotyped bobcats, MKA was used to develop relative minimum densities for comparisons of relative abundance between ecoregions and the Royle-Nichols model was used to estimate the actual census size, which was 10,926.51 \pm 2,960.43, with a 95\% confidence interval of 6,018.11 - 19,838.26. However, the Royle-Nichols model may be slightly overestimating abundance, due to the decreased detection probabilities in some locations of West Virginia where bobcat occupancy is sparse.

The overall genotyping success rate of this study (60.8\%) is comparable to the $56 \%$ success rate of the only other recent non-invasive study of bobcats using hair snares (Stricker et al. 2012). The success rate of amplifying genotypes from scat samples at 4 microsatellite loci was also similar at 41\% (Morin et al. 2018). While this study had greater genotyping success, it is probably due to the use of a smaller number of microsatellites. The three microsatellite loci used in this project to first screen all samples were highly polymorphic so these genotype profiles had ample power to screen between individuals without introducing the potential error from null alleles and allelic dropout at each additional locus. As compared to these other studies where capture-recapture was used for final population estimation, this study used raw data. A consequence of this is that the missing samples, either from imperfect detection, or those lost in genotyping, impacted the final outcome of the population estimates. The numbers herein are used as minimum values, and the actual census densities are expected to be relatively larger. The MKA values for density should be used as the minimum bound for any census estimates. 
Of the six ecological regions of West Virginia, ecoregion 4, or north-central West Virginia had both the highest number of bobcat detections and the greatest estimated minimum bobcat density. While this relationship may have been driven partially by the comparatively large number of detections at the Braxton County site in 2015, the overall numbers for this ecological region are roughly twice that of any other ecological region. Plus, nearly all ecological regions except for 4 had similar numbers of detections. While ecoregion 4 is mostly forested, it has more agricultural disturbance than locations like the Monongahela National Forest (ecoregion 2). This landscape disturbance is thought to increase the local prey diversity and abundance, which in turn, allows for greater bobcat densities, as opposed to monotypic habitats being specifically selected by bobcats (McCord 1974, Litvaitis et al. 1986, Chamberlain et al. 1999, 2003, Long et al. 2011, Lesmeister et al. 2015). Ecoregion 4 has conditions that appear optimal for bobcat population growth: contiguous forest for cover interspersed with areas of early successional habitat and enhanced hunting opportunity. The location with the second greatest minimum number of animals was located in Boone County in ecoregion 3. However, this particular site was located on and adjacent to former mining lands that are in the process of reclamation. These early successional habitats interspersed by mature woodlands again appear to be a factor in the increased bobcat density at this location as compared to the rest of ecoregion 3.

Once accounting for error due to imperfect detection, the average minimum densities, as calculated for each ecoregion, are similar to actual densities that were found in other localities using capture-recapture techniques. This indicates that the actual census size of the West Virginia bobcat population is likely much greater than these locations. In the Upper Peninsula of Michigan, the bobcat density was calculated at $3.0 / 100 \mathrm{~km}^{2}$ (Stricker et al. 2012), in central Wisconsin it was averaged at $2.53 / 100 \mathrm{~km}^{2}$ (Clare et al. 2015), and most recently in neighboring 
Virginia, density estimates ranged from $6.65-25.84$ / 100km², with a mean of 12.5 individuals / $100 \mathrm{~km}^{2}$ (Morin et al. 2018). These density estimates are similar to what was calculated for individual sites, both before and after accounting for the detection probabilities. The interpolated output of the Royle-Nichols model estimated the census size of West Virginia's bobcat population at 10,926.51 individuals. If these bobcats were distributed evenly across the suspected $59,500 \mathrm{~km}^{2}$ of bobcat habitat the average density would be 18.36 bobcats $/ 100 \mathrm{~km}^{2}$, with each bobcat having a roughly $5.44 \mathrm{~km}^{2}$ home range, which is much smaller than expected. However, yearling and juvenile individuals are expected to have smaller home ranges than adults and they were found to comprise roughly 50\% of the overall population (Landry 2017). If these yearlings and juveniles, on average, have home range sizes that are between $35-50 \%$ of an adult, the average adult female home range size is predicted to be roughly $8-10 \mathrm{~km}^{2}$. The lack of spatial recaptures in this study may also be a result of the fact that most bobcats were only ever exposed to a single sampling device located in their home range. If this is true, then the average adult home range size is more likely to be $10 \mathrm{~km}^{2}$, as opposed to the $20 \mathrm{~km}^{2}$ for females assumed at the beginning of this study.

The census value estimated by the Royle-Nichols model likely has a slight upwards bias, due to the reduced secondary capture rate for a bobcat after it had already been sampled once, which is a violation of an assumption of the model. The overall impact of violating this assumption is unknown and the model will have to be validated empirically by future research. However, the use of 1,275 individual $10 \mathrm{~km}^{2}$ sites in constructing this model will reduce the impact of this potential bias.

While average density values may be useful for management, the results of this study show that density values vary tremendously between different locations and habitat types. Also, 
the number of bobcats on the landscape appeared to change significantly between the 2015 and 2016 field seasons. This trend was not limited to bobcats, either, as the number of black bear (Ursus americanus) disturbance events recorded at hair snare cubbies in 2016 was roughly 10\% of that in 2015. This reduction of the bobcat population in 2016 is likely the result of reduced juvenile survival, which was observed by Landry (2017) when analyzing the 2015 - 2016 bobcat harvest data.

The relatively low recapture rate of $4 \%$ for this study is disconcerting since it limits the usefulness of the bobcat hair snare cubby in SECR analyses. However, it is also possible that this low number of recaptures is reflective of an overall high density of bobcats on the landscape since the cubby avoidance rate is unknown. Hair snare studies of bobcats commonly have low capture and recapture rates (Long et al. 2007, Ruell and Crooks 2007, Comer et al. 2011, Stricker et al. 2012). The sites that were sampled twice (REDOs) demonstrated the prevalence of bobcat “trap shyness” to the cubbies after the initial sampling event. Sites like the Boone County location, from which 16 unique individuals were detected in 2015, and only two in 2016, provide compelling evidence that bobcats actively avoid being resampled, even when they are known to be present. The sites that were redone, in general, only recorded a small fraction of the samples as compared to their original sampling (appendix Table 1). The failure to achieve sufficient recaptures, however, does not mean that this method is not worthwhile. In maintain the interest of bobcats that have already been sampled, different scent baits should be used between the sampling weeks. Additionally, the use of new visual attractants could make individuals curious enough to reenter the sampling device. With some modification, the bobcat hair snare cubby should become an increasingly valuable asset for collecting samples specifically for use in population estimation. 
As estimated by the Royle-Nichols model the actual population size of West Virginia bobcats was 10,926.51 $\pm 2,960.43$, with a 95\% confidence interval of 6,018.11 - 19,838.26. Using a conservative interpretation of Crowe’s (1975) model for exploited bobcat populations and data from Fox and Fox (1982), a yearly surplus of 1.26 bobcats per individual (21\%) is produced in West Virginia. As long as the harvest remains below this level, the bobcat population will grow or, at minimum, remain stable. During the $2014-2015$, and the 2015 2016 hunting and trapping seasons in West Virginia, 1,898 bobcats and 1,346 bobcats, respectively were harvested. In order to remain below the target $21 \%$ harvest threshold, the population size needed to be at least 9,038 individuals to sustain a harvest of 1,898 and 6,410 to sustain a harvest of 1,346 individuals.

If the Royle-Nichols estimate of the population size is correct, then the current population is of sufficient size to sustain harvests as occurred in the 2014 - 2015 hunting and trapping season. However, when taking into account the standard error of the Royle-Nichols population estimate, it is possible that the population size is as low as roughly 7,966.08 animals. A harvest of 1,898 individuals is roughly $23 \%$ of the total population and may lead to a decline using the conservative $21 \%$ yearly surplus. If the true population size is at the lower end of this range, then harvests should be limited to roughly 1,700 individuals per season to maintain a growing or stable population. Additional study in the form of bobcat trapping and radio-collaring should help to reduce the error range of the current population estimate. In regard to the efficacy of the current management strategy for bobcats in West Virginia, an independent analysis using the Crowe model and habitat covariates estimated the bobcat population size to be roughly 7,500 10,000 (personal communication: Rich Rogers, WVDNR). The population estimates produced by this study provided evidence that the Crowe model is a useful predictive tool for the West 
Virginia bobcat population; however, it should be updated with the new demographic data as calculated by Landry (2017).

\section{Management Implications}

The bobcat hair snare cubby is a successful device for collecting hair samples from bobcats with adequate quality for individual identification. However, the overall recapture rate of the cubby was considered lower than expected and steps should be taken to change the scent and visual attractants used between sampling weeks. The Royle-Nichols model generated a population estimate similar to West Virginia's currently used bobcat management model. Once updated with current demographic data, this model should be sufficient to ensure sustainable bobcat management in West Virginia. Until an empirical evaluation of the population estimate produced by this study can be undertaken, an increase in bobcat harvest is inadvisable and a yearly harvest limit of, on average, 1,700 individuals should result in a stable or growing bobcat population.

Another significant finding of this study is that bobcats are not evenly distributed across the landscape. Densities were greatest in ecoregion 4, or north-central West Virginia. Bobcats were also found to be clumped in locations of assumed greater prey densities, even in ecoregions with overall lower bobcat abundances. The density of bobcats in these locations makes the previously estimated average home range size of $20 \mathrm{~km}^{2}$ appear incorrect. The average adult bobcat home range size, at least for females, appears to be closer to $10 \mathrm{~km}^{2}$.

Finally, some of the most highly productive locations for bobcats could support higher harvest rates than a blanket statewide approach to management; however, differing harvest rates throughout the state may create logistical complications. 


\section{Acknowledgements}

We thank the many landowners of West Virginia for allowing us to sample their properties for bobcats, as well as the many members of the West Virginia Division of Natural Resources that worked on this project and made such a large-scale data collection effort possible_-especially Steve Rauch, Rob Silvester, Colin Carpenter, Eric Richmond, and Jeff McCrady. We also thank Avery Owers, Brent Mays, Lauren Schumacher, Jarrod Ball, Brittany Demski, Lauren Schumacher, Brittney Watson, Peter Ries, Steven Wilson, Matt Stevens, Shelby Sherman, Kyle Matt, Sheila Harris, Darren Wood, and Paige Pritt of West Virginia University for their assistance in collecting hair samples from gun brushes and processing them in the laboratory. Financial support for this dissertation was provided by the West Virginia Division of Natural Resources through a grant with the U.S. Fish and Wildlife Service Wildlife and Sport Fish Restoration Program (W-48-R) and matching state funds. 


\section{References}

Borchers, D. L., and M. G. Efford. 2008. Spatially Explicit Maximum Likelihood Methods for Capture-Recapture Studies. Biometrics 64:377-385.

Chamberlain, M. J., B. D. Leopold, L. W. Burger, B. W. Plowman, and L. M. Conner. 1999. Survival and Cause-Specific Mortality of Adult Bobcats in Central Mississippi. The Journal of Wildlife Management 63:613.

Chamberlain, M. J., B. D. Leopold, and L. M. Conner. 2003. Space Use, Movements and Habitat Selection of Adult Bobcats (Lynx rufus) in Central Mississippi. The American Midland Naturalist 149:395-405.

CITES $17^{\text {th }}$ Meeting. 2016. Seventeenth Meeting of the Conference of the Parties. Johannesburg, South Africa.

Clare, J. D. J., E. M. Anderson, and D. M. MacFarland. 2015. Predicting bobcat abundance at a landscape scale and evaluating occupancy as a density index in central Wisconsin. The Journal of Wildlife Management 79:469-480.

Comer, C. E., M. E. Symmank, and J. C. Kroll. 2011. Bobcats Exhibit Low Detection Rates at Hair Collection Stations in East Texas. Wildlife Biology in Practice 7:116-122.

Crowe, D. M. 1975. A Model for Exploited Bobcat Populations in Wyoming. The Journal of Wildlife Management 39:408.

Erb, P. L., W. J. McShea, and R. P. Guralnick. 2012. Anthropogenic Influences on Macro-Level Mammal Occupancy in the Appalachian Trail Corridor. PLOS ONE 7:e42574.

Fox, L. B., and J. S. Fox. 1982. Population Characteristics and Food Habits of Bobcats in West Virginia. Proceedings of the Annual Conference of the Southeast Association of Fish and Wildlife Agencies.

Hines J.E. (2006) PRESENCE-Software to Estimate Patch Occupancy and Related Parameters. Available: http://www.mbr-pwrc.usgs.gov/software/presence.html.

Kendall, K. C., K. S. McKelvey, and others. 2008. Hair collection. Noninvasive survey methods for carnivores. Island Press, Washington, DC 141-182.

Knecht, L., T.F. Rounsville Jr., and J.E. Huffman. 2015. Dynamic Hair Identification Key Ver. 1.0B. http://www.hairdatabase.com.

Lesmeister, D. B., C. K. Nielsen, E. M. Schauber, and E. C. Hellgren. 2015. Spatial and Temporal Structure of a Mesocarnivore Guild in Midwestern North America. Wildlife Monographs 191:1-61. 
Linden, D. W., A. K. Fuller, J. A. Royle, and M. P. Hare. 2017. Examining the OccupancyDensity Relationship for a Low-density Carnivore. Journal of Applied Ecology 54:20432052.

Litvaitis, J. A., J. A. Sherburne, and J. A. Bissonette. 1986. Bobcat Habitat Use and Home Range Size in Relation to Prey Density. The Journal of Wildlife Management 50:110.

Landry, S. M. 2017. Bobcat Population Ecology in West Virginia. M.S. Thesis. West Virginia University. pp. 1-179.

Lombardi, J. V., C. E. Comer, D. G. Scognamillo, and W. C. Conway. 2017. Coyote, Fox, and Bobcat Response to Anthropogenic and Natural Landscape Features in a Small Urban Area. Urban Ecosystems 20:1239-1248.

Long, R. A., T. M. Donovan, P. Mackay, W. J. Zielinski, and J. S. Buzas. 2007. Comparing Scat Detection Dogs, Cameras, and Hair Snares for Surveying Carnivores. The Journal of Wildlife Management 71:2018-2025.

Long, R. A., T. M. Donovan, P. MacKay, W. J. Zielinski, and J. S. Buzas. 2011. Predicting carnivore occurrence with noninvasive surveys and occupancy modeling. Landscape Ecology 26:327-340.

Lovallo, M. J., and E. M. Anderson. 1996. Bobcat (Lynx rufus) Home Range Size and Habitat Use in Northwest Wisconsin. American Midland Naturalist 135:241.

Menotti-Raymond, M. A., and S. J. O’Brien. 1995. Evolutionary Conservation of Ten Microsatellite Loci in Four Species of Felidae. Journal of Heredity 86:319-322.

Menotti-Raymond, M., V. A. David, L. A. Lyons, A. A. Schäffer, J. F. Tomlin, M. K. Hutton, and S. J. O’Brien. 1999. A Genetic Linkage Map of Microsatellites in the Domestic Cat (Felis catus). Genomics 57:9-23.

Menotti-Raymond, M., V. A. David, J. C. Stephens, L. A. Lyons, and S. J. O’Brien. 1997. Genetic Individualization of Domestic Cats Using Feline STR Loci for Forensic Applications. Journal of Forensic Sciences 42:1039-1051.

McCord, C. M. 1974. Selection of Winter Habitat by Bobcats (Lynx rufus) on the Quabbin Reservation, Massachusetts. Journal of Mammalogy 55:428.

McCord, C.M. and J.E. Cardoza. 1982. Bobcat and Lynx. Pages 728-766 in J.A. Chapman and G.A. Feldhammer, eds. Wild mammals of North America: biology, management, and economics. Johns Hopkins Univ. Press, Baltimore, Md.

Morin, D. J., L. P. Waits, D. C. McNitt, and M. J. Kelly. 2018. Efficient single-survey estimation of carnivore density using fecal DNA and spatial capture-recapture: a bobcat case study. Population Ecology 60:197-209. 
Progulske, D.R. 1952. The Bobcat and its Relation to Prey Species in Virginia. M.S. Thesis. Virginia Poly. Inst., Blacksburg, Va. 135 pp.

Roberts, N. M., and S. M. Crimmins. 2010. Bobcat Population Status and Management in North America: Evidence of Large-Scale Population Increase. Journal of Fish and Wildlife Management 1:169-174.

Royle, J. A., and J. D. Nichols. 2003. Estimating Abundance from Repeated Presence-Absence Data or Point Counts. Ecology 84:777-790.

Royle, J. A., R. B. Chandler, R. Sollmann, and B. Gardner. 2013. Spatial Capture-Recapture. Academic Press.

Ruell, E. W., and K. R. Crooks. 2007. Evaluation of Noninvasive Genetic Sampling Methods for Felid and Canid Populations. The Journal of Wildlife Management 71:1690-1694.

Stricker, H. K., J. L. Belant, D. E. Beyer, J. Kanefsky, K. T. Scribner, D. R. Etter, and J. Fierke. 2012. Use of modified snares to estimate bobcat abundance. Wildlife Society Bulletin 36:257-263.

Thornton, D. H., and C. E. Pekins. 2015. Spatially explicit capture-recapture analysis of bobcat (Lynx rufus) density: implications for mesocarnivore monitoring. Wildlife Research 42:394.

Wang, Y., M. L. Allen, and C. C. Wilmers. 2015. Mesopredator Spatial and Temporal Responses to Large Predators and Human Development in the Santa Cruz Mountains of California. Biological Conservation 190:23-33.

White, G. C. 1982. Capture-recapture and removal methods for sampling closed populations. Los Alamos National Laboratory.

Wilberg, M. J., and B. P. Dreher. 2004. genecap: a program for analysis of multilocus genotype data for non-invasive sampling and capture-recapture population estimation. Molecular Ecology Notes 4:783-785. 


\title{
Chapter 5 - Population Genetics of West Virginia's Bobcats
}

\begin{abstract}
Knowledge of population structure, effective population size $\left(\mathrm{N}_{\mathrm{e}}\right)$, and occurrence of a recent genetic bottleneck can be useful when designing management strategies for wildlife. However, these basic genetic data are often unavailable, even for species that are regularly harvested. In West Virginia, spleen samples were collected from 304 bobcat (Lynx rufus) carcasses donated during the 2014 - 2015 hunting and trapping season. These samples were then examined with nine polymorphic microsatellites for the presence of population structure, to estimate $\mathrm{N}_{\mathrm{e}}$, and to determine if a recent bottleneck event occurred for each identified population. A total of $92.1 \%(n=280)$ of bobcat spleens produced microsatellite genotypes at $\geq$ 5 loci and were included in the final analyses. Three separate methods (STRUCTURE, a PCA, and TESS) failed to produce any biologically relevant evidence of population structure. Further analyses were conducted condensing the bobcats of West Virginia as one larger population. The effective population size $\left(\mathrm{N}_{\mathrm{e}}\right)$ of West Virginia's bobcat population was 1,448.7, with a 95\% parametric confidence interval of 535.6 - $\infty$ and a jackknifed $95 \%$ confidence interval across all loci of $426.8-\infty$. Both the Sign Test and the Wilcoxon one-tailed test for heterozygote deficiency supported a recent, statistically significant population bottleneck in West Virginia’s bobcats. Due to the lack of genetic population structure, bobcats in West Virginia may continue to be managed as one, large population.
\end{abstract}




\section{Introduction}

Genetic diversity is important to long-term viability of wildlife populations since it provides a mechanism to continually evolve in a constantly changing environment (Lacy 1997). Lack of sufficient genetic diversity can reduce the overall fitness of a population by increasing susceptibility to disease, causing developmental defects, or leading to deleterious recessive alleles to accumulate in homozygous form. In small, isolated populations, genetic drift causes genetic diversity to be lost much more quickly than in larger populations (Allendorf 1986). While the potential negative impacts that the lack of genetic diversity can have on a wildlife population have been documented, information on local genetic variation does not exist for many species that are regularly harvested, such as bobcats (Lynx rufus) in West Virginia. Also, these animals are thought to have undergone a severe population reduction during the late 1950s and 1960s (personal communication, Rich Rogers, West Virginia Division of Natural Resources), which may have resulted in a long-lasting genetic impact.

The first nearly range-wide study to sample West Virginia bobcats used a combination of mitochondrial DNA (mtDNA) haplotyping and genotyping at 15 microsatellite loci to investigate patterns of genetic differentiation (Reding et al. 2012). At the continental scale of North America, West Virginia bobcats were classified as most similar to those from the southeastern United States; however, genetic differentiation between West Virginia and Pennsylvania was observed. When comparing the eastern and western United States populations, Reding et al. (2012) found that western bobcats were much more genetically homogenous than those in the east. This was hypothesized to be a result of the greater anthropogenic habitat impacts in the eastern United States and that bobcats in the east were much more patchily distributed. Another 
rangewide study also reached the same conclusion when sampling bobcats at the landscape scale (Croteau et al. 2012).

At the local scale, only Anderson et al. (2015) have studied the genetic variation of West Virginia bobcats, and only in the context of examining putative populations of origin for the recovering population of bobcats in Ohio. While the overall sample numbers from different localities were small, Anderson et al. (2015) found that West Virginia’s bobcats were genetically distinct from those of eastern Ohio, but similar to samples collected from western Kentucky, southern Ohio, and western Pennsylvania. When examined together, the southern OH, WV, western and eastern KY, and western PA group was found to have undergone a genetic bottleneck event, indicative of their historic population reduction and recent recovery.

Anthropogenic landscape features have also been shown to significantly impact bobcat gene flow, which may be an issue in West Virginia. Primary highways have been identified as major barriers to gene flow (Riley et al. 2006, Lee et al. 2012, Ruell et al. 2012, Serieys et al. 2015). A meta-analysis of population genetic studies of many carnivore species found that fenced highways are a major barrier to wildlife movement (Holderegger and Di Giulio 2010). However, roads may not always have a significant impact on bobcat gene flow (Millions and Swanson 2007) and even highways may not be a complete barrier (Holderegger and Di Giulio 2010). In the absence of anthropogenic barriers, genetic differentiation between bobcats sampled in different locations remains relatively low, as was observed in southern Illinois (Croteau et al. 2010) and the interconnected region between Georgia and Florida (Reid 2006). Unless major interstate highways or urbanized areas of West Virginia are functioning as significant barriers, it is expected that bobcat gene flow across the state will be sufficient to remove any signatures of population structure. 
To make the most informed conservation or management decisions, delineation of genetic population structure is important. The identification of groups that are genetically distinct, even in contiguously distributed species, is needed to ensure that the evolutionary potential of these species is conserved (Moritz 1994). Knowledge of these separate population clusters can also allow for the putative identification of areas where gene flow is significantly reduced. Management or conservation actions can then be proposed to mitigate these barriers and restore gene flow to the landscape.

\section{Study Objectives}

The objectives of this research were to: 1) identify the number of genetically distinct bobcat populations in West Virginia; 2) use identified genetically distinct population clusters to investigate anthropogenic or landscape features of West Virginia that may be barriers to gene flow; 3) calculate basic population genetic parameters for identified populations which may provide additional information on the biology of the species; 4) estimate effective population sizes $\left(\mathrm{N}_{\mathrm{e}}\right)$ for each identified population; 5) determine whether or not there is genetic evidence of a recent population bottleneck in West Virginia bobcats.

\section{Materials and Methods}

\section{Sample Collection}

During the 2014 - 2015 hunting and trapping seasons (Nov. - Feb.) the West Virginia Division of Natural Resources (WVDNR) collected bobcat carcasses donated by successful hunters and trappers. These carcasses were the source of data for this and other portions (Landry 2017) of the WVDNR bobcat project. A necropsy was completed on each of the 304 donated 
bobcat carcasses and entire bobcat spleens were removed for the population genetic analyses as well as Carnivore protoparvovirus 1 testing (Chapter 6). Once removed from the carcasses, the bobcat spleens were stored individually at $-20^{\circ} \mathrm{C}$ until DNA extraction.

\section{DNA Extraction and Standardization}

DNA extractions were begun by thawing the spleen samples collected at necropsy and removing a 10 - 15mg subsample from the interior of the tissue with a razor blade. The same blade was used for multiple samples, but it was cleaned thoroughly with a laboratory wipe coated in 95\% ethanol after each collection. Subsamples were placed into a 1.5mL microcentrifuge tube in preparation for use with the ThermoFisher (Pittsburgh, PA) GeneJet Tissue and Cells DNA Extraction Kit. The manufacturer’s suggested protocol was used for the DNA extraction procedures, except that samples were only incubated at $56^{\circ} \mathrm{C}$ for 1.5 hours, and $30 \mu \mathrm{L}$ of $1 \mathrm{M}$ DTT was added to each sample to assist in denaturation of splenic proteins. Once completed, the DNA extracts were normalized to a 10ng/ $\mu \mathrm{L}$ concentration using a ThermoFisher NanoDrop Lite spectrophotometer.

\section{PCR and Resolution of DNA Fragments}

Microsatellite genotype profiles were constructed for each bobcat sample using ten microsatellite loci: FCA008, FCA23, FCA26, FCA43, FCA45, FCA77, FCA90 (MenottiRaymond and O’Brien 1995, Menotti-Raymond et al. 1997, 1999), 6HDZ057, 6HDZ463, and 6HDZ700 (Williamson et al. 2002). These microsatellite loci were amplified using a series of three multiplex reactions: A (FCA90, FCA23, and FCA26), B (6HDZ057, 6HDZ463, FCA 008, and FCA77), and C (FCA43, 6HDZ700, and FCA45). Total reaction volume for each multiplex 
PCR was $10 \mu \mathrm{L}$ and consisted of $5 \mu \mathrm{L}$ of the 2x Qiagen Multiplexing Master Mix (Germantown, MD), 330nmol (A, C) or 260nmol (B) of each individual primer, and 20ng of DNA template. PCR reaction conditions were as follows: 15 -minute hot-start at $95^{\circ} \mathrm{C}, 35$ cycles of $94^{\circ} \mathrm{C}$ for 30 s, $57^{\circ} \mathrm{C}$ for 90 s, and $72^{\circ} \mathrm{C}$ for 90 s, with a final extension of $72^{\circ} \mathrm{C}$ for 10 minutes. Amplified DNA fragments were resolved on a Beckman-Coulter GeXP Genetic Analysis System (AB SCIEX, Washington, D.C.) and fragment sizes were recorded using the associated GeXP fragment analysis software. Samples failing to amplify at least 5 microsatellite loci were eliminated from further analysis to reduce the potential of low-quality samples biasing the results. Genotypes constructed for each remaining sample were screened with MICRO-CHECKER (Van Oosterhout et al. 2004) to find and eliminate genotyping errors.

\section{Genetically Distinct Population Identification}

Before additional analyses were completed, the first step was to determine the most likely number of genetically distinct populations in West Virginia. Once this was determined, all subsequent analyses were applied to each individual population that was discovered. The program STRUCTURE (Pritchard et al. 2000, Falush et al. 2003, Falush et al. 2007) was used to investigate the presence of genetic population structure in West Virginia's bobcats. Ten iterations of a 10,000 repetition burn-in followed by 100,000 Markov chain Monte Carlo (MCMC) repeats of the admixture correlated model was completed for each hypothesis for $K$ (the number of distinct population clusters) from $1-10$. The output models of STRUCTURE were then analyzed by STRUCTURE Harvester (Earl and vonHoldt 2012) to determine the most likely value of $K$. 
The second method used to elucidate population genetic structure was a principal components analysis (PCA). GenAlEx v.6.503 (Peakall and Smouse 2012) was used to calculate a matrix of Nei's pairwise genetic distance for each individual microsatellite locus and a combined pairwise genetic distance across all loci. A PCA analysis was then undertaken on these combined pairwise genetic distances using the standardized-distance PCA method of GenAlEx.

The third method used to investigate potential genetic population structure was TESS v2.3 (Chen et al. 2007, Durand et al. 2009), which uses a similar Bayesian clustering algorithm as STRUCTURE that makes assignments that minimize HWE deviations, but takes into account the physical landscape location from which each sample was collected when making these assignments. To calculate geographical assignments of each collected sample, ArcMap v.10.4 (ESRI, Redlands, CA) was used to create centroids of each of the counties from which bobcat carcasses were donated. Each genotype profile was then associated with the centroid point of the county of its origin in geographical space. These combined data were then used in TESS v2.3 to create geographically explicit population models using the admixture correlated model and a kmax (maximum number of hypothesized populations) of 10. A total of 50 iterations were run for each hypothesis of kmax and output files were combined using CLUMPP v1.1.2 (Jakobsson and Rosenberg 2007) to evaluate overall trends in population assignment probabilities for each sample.

\section{Basic Population Genetic Parameters}

The program CERVUS v3.0 (Kalinowski et al. 2007) was used to calculate basic population genetic parameters for each amplified microsatellite locus, including the unique number of alleles $(\mathrm{k})$, the observed $\left(\mathrm{H}_{\mathrm{O}}\right)$ and expected $\left(\mathrm{H}_{\mathrm{E}}\right)$ heterozygosity, and whether or not 
the genotype frequencies of each amplified locus were within the expectations of HardyWeinberg Equilibrium (HWE). CERVUS v3.0 calculates significance for HWE testing using a standard Bonferroni correction, where the value of $\alpha$ is divided by the overall number of tests, which in this case is the total number of microsatellite loci. Before the Bonferroni correction, the value of $\alpha$ was selected as 0.05 .

\section{Bottleneck Analysis}

The program BOTTLENECK (Piry et al. 1999) was used to investigate whether or not each identified West Virginia bobcat population was subjected to a recent genetic bottleneck event. This program examines the current levels of heterozygosity observed for each microsatellite locus $\left(\mathrm{H}_{\mathrm{O}}\right)$ and compares it to the heterozygosity expected based on the selected mutation model, combined with the expected value of the mutation-drift equilibrium. For this analysis, the two-phase mutation model ( $70 \%$ stepwise) was selected since it is thought to be the most representative for microsatellites. A total of 10,000 iterations were completed for both the sign test and the Wilcoxon sign rank test, with $\alpha=0.05$.

\section{Effective Population Size Analysis}

To calculate the effective population size for each identified West Virginia bobcat population, the program LDNe (Waples and Do 2008) was used. LDNe estimates the effective population size by first calculating the amount of linkage disequilibrium (LD) by using Burrows' $\Delta$ and then averaging across all allele combinations and loci. Thus, LDNe provides an effective way to estimate the average population size from microsatellite data without additional required information. For the analyses, only alleles with a frequency $\geq 0.02$ were considered, the random 
mating model was used, and both the parametric and jackknife confidence intervals were calculated.

\section{Results}

Of the 304 bobcat carcasses donated by hunters and trappers from the 2014 - 2015 season, $92.1 \%(n=280)$ produced genotypes at $\geq 5$ loci and were used for the subsequent analyses. However, microsatellite locus 6HDZ463 failed to amplify for $>50 \%$ of the remaining 280 samples and was removed from the study to avoid potential bias, dropping the total number of microsatellite loci analyzed in this study to nine. These constructed genotypes were then successfully used by the program STRUCTURE to investigate the most likely number of genetically distinct populations in this dataset. The analysis of the STRUCTURE output files by STRUCTURE Harvester revealed that the most likely number of populations for this dataset was $K=1$, since it had the greatest likelihood, or $\operatorname{LnP}(K)$, as compared to the other models (Figure 11), and little variance between iterations. Even though the value of $\Delta K$ was greatest for $K=3$, the putative population assignments of STRUCTURE, when viewed graphically, did not provide support for this hypothesis. Most individuals were nearly equally assigned to each of the three suggested populations (Figure 12a). A similar pattern was discovered when examining the population assignment probabilities for the other tested hypotheses of $K$, such as $K=2$ (Figure 12b). Since the $K=1$ hypothesis produced the greatest value of $\operatorname{LnP}(K)$, and no evidence of population differentiation was found in the graphical assignments, $K=1$ was determined to be the most likely hypothesis based on the STRUCTURE output. 


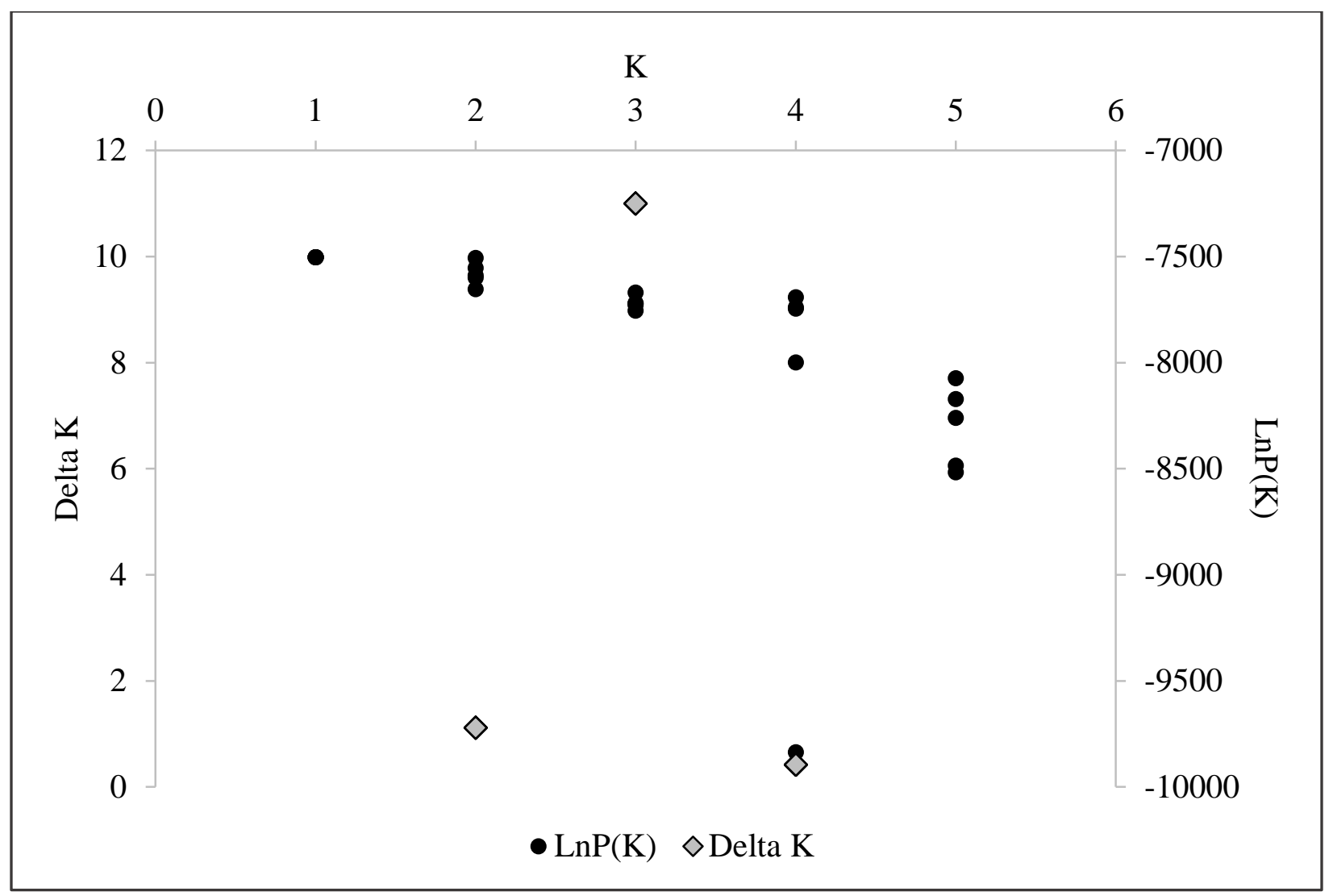

Figure 11 - Graph of $\Delta K$ and log likelihood $(\operatorname{LnP}(K))$ for each value of $K$ (hypothesized number of distinct genetic clusters) for 280 West Virginia bobcat samples genotyped at nine microsatellite loci. This graph was produced as the output of STRUCTURE Harvester (Earl and vonHoldt 2012) after analyzing the ten iterations completed for each value of $\mathrm{k}$ from $1-10$. However, values of $K>5$ were not graphed by STRUCTURE Harvester due to their significantly lower $\mathrm{LnP}(K)$ values. 

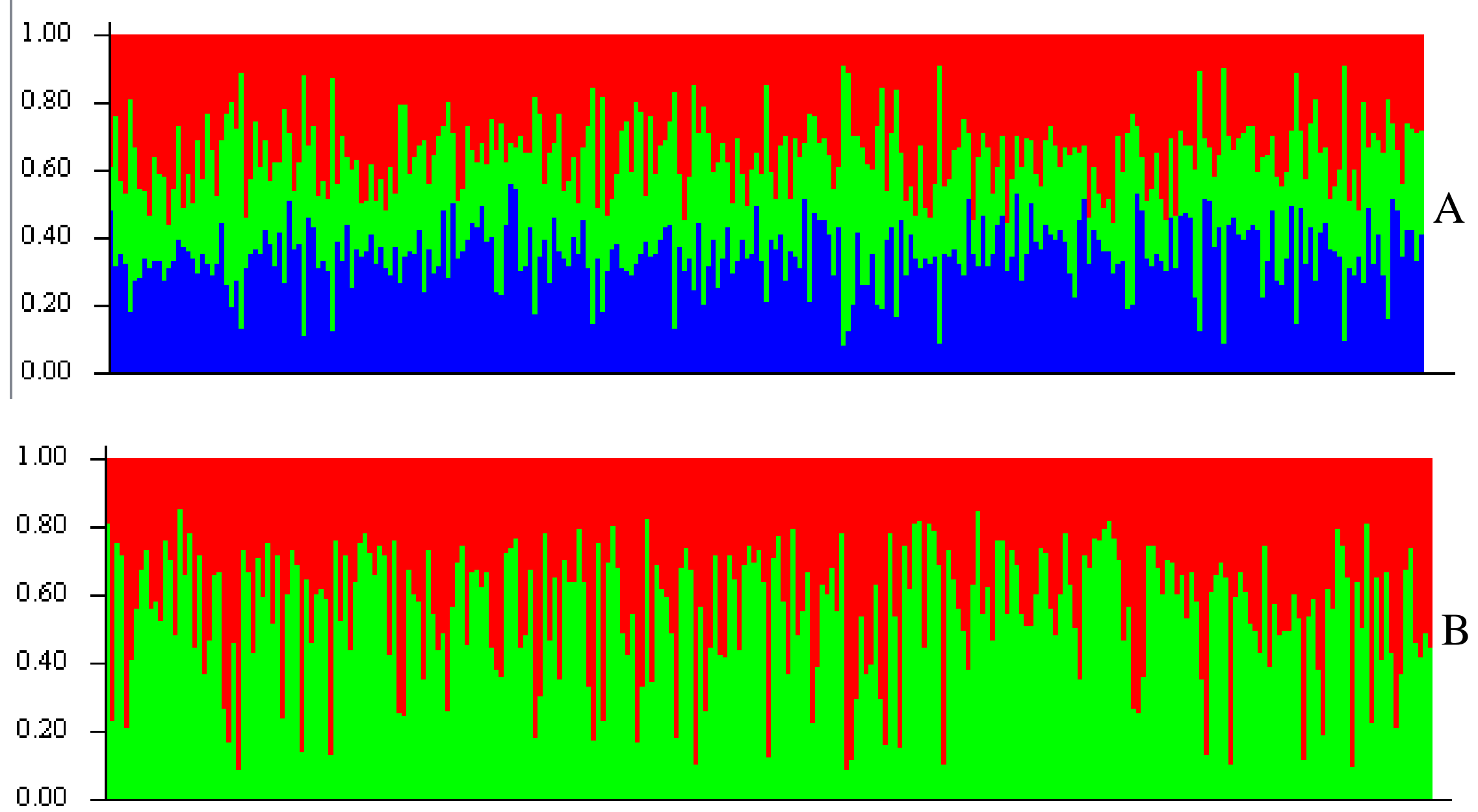

Figure 12 (A \& B) - Output of the program STRUCTURE (Pritchard et al. 2000, Falush et al. 2003, Falush et al. 2007) when $K=3$ (a) and $K=2$ (b) for the 280 bobcats of West Virginia analyzed at nine microsatellite loci. Each bar represents one individual. The height of each color on the bar is the probability of assignment for that individual to that particular genetic cluster. 
The PCA completed on the pairwise genetic distance produced three distinct genetic clusters; however, $85.4 \%$ of the total samples $(\mathrm{n}=239)$ were placed into one tight cluster (Figure 13). When the samples comprising the two outlying clusters of the PCA were plotted geographically, the distribution was found to be random across West Virginia. Thus, the PCA also failed to provide supporting evidence for $K=3$, because these groupings had no biological support. Analysis of TESS output revealed the most likely hypothesis for $K$ to be $K=5$, since this value was located below the inflection point of the plotted curve for the Deviance Information Criterion (DIC) plot (Figure 14). When examined graphically, population assignment probabilities were similar to that of STRUCTURE, with most individuals assigned to one group with a few outliers with multiple, nearly equal assignments (Figure 15). The failure of TESS to also find any biologically meaningful genetic population structure also supports the hypothesis that $K=1$ for the bobcats of West Virginia. Each of the three methods used on this dataset failed to find any meaningful population genetic structure, and thus, $K=1$, or complete panmixia, is the best supported hypothesis. All subsequent analyses were completed considering West Virginia bobcats to be members of a single, large population. 


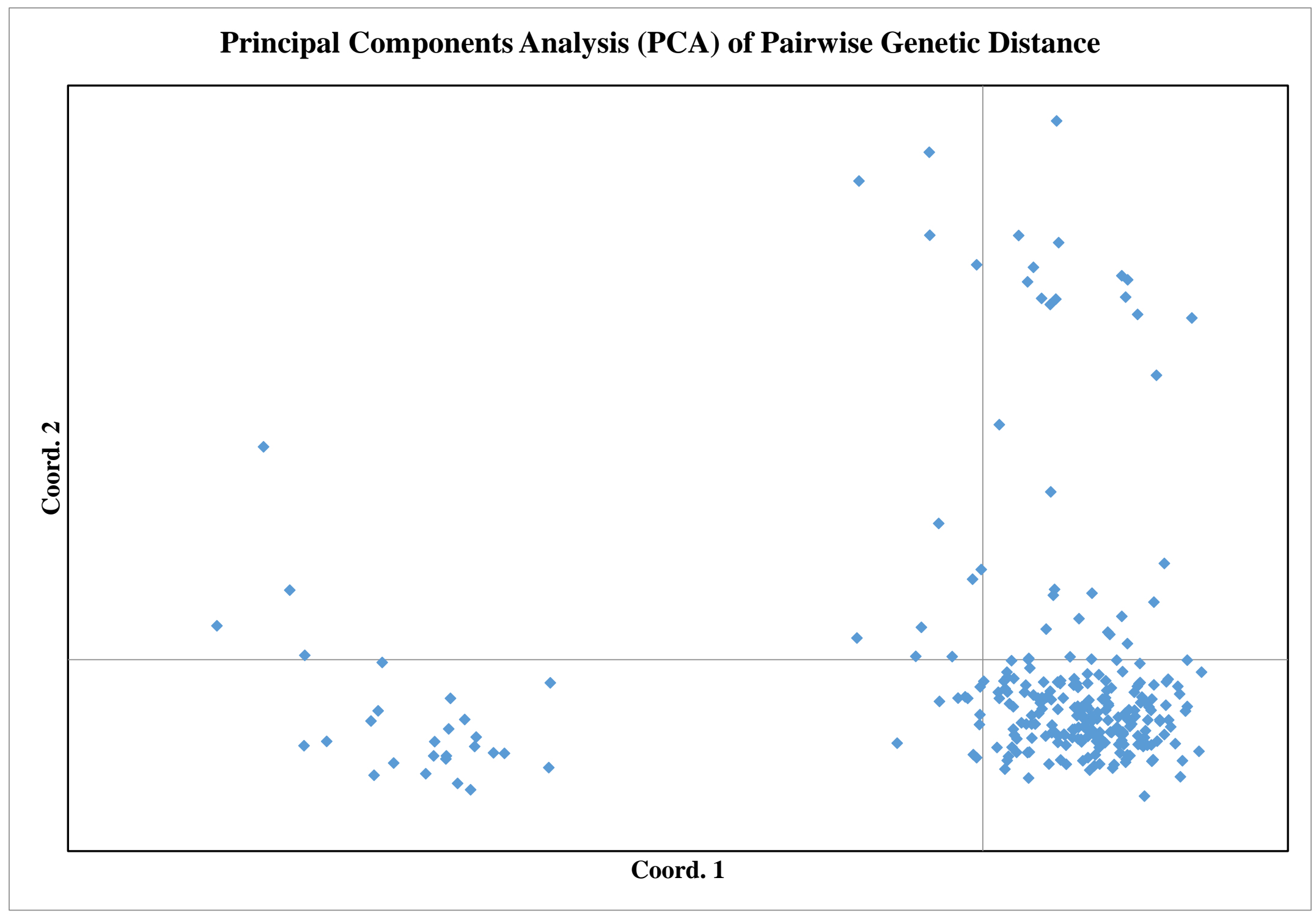

Figure 13 - Principal Components Analysis (PCA) of Nei’s pairwise genetic distance as calculated as a combined score across nine microsatellite loci from 280 bobcat samples from West Virginia. This PCA analysis was completed in GenAlEx v.6.503 (Peakall and Smouse 2012). Axis 1 contained 10.53\% of all variation and axis 2 contained 9.11\% of all variation, for a total of $19.64 \%$ of variation explained by the combination of these two axes. 


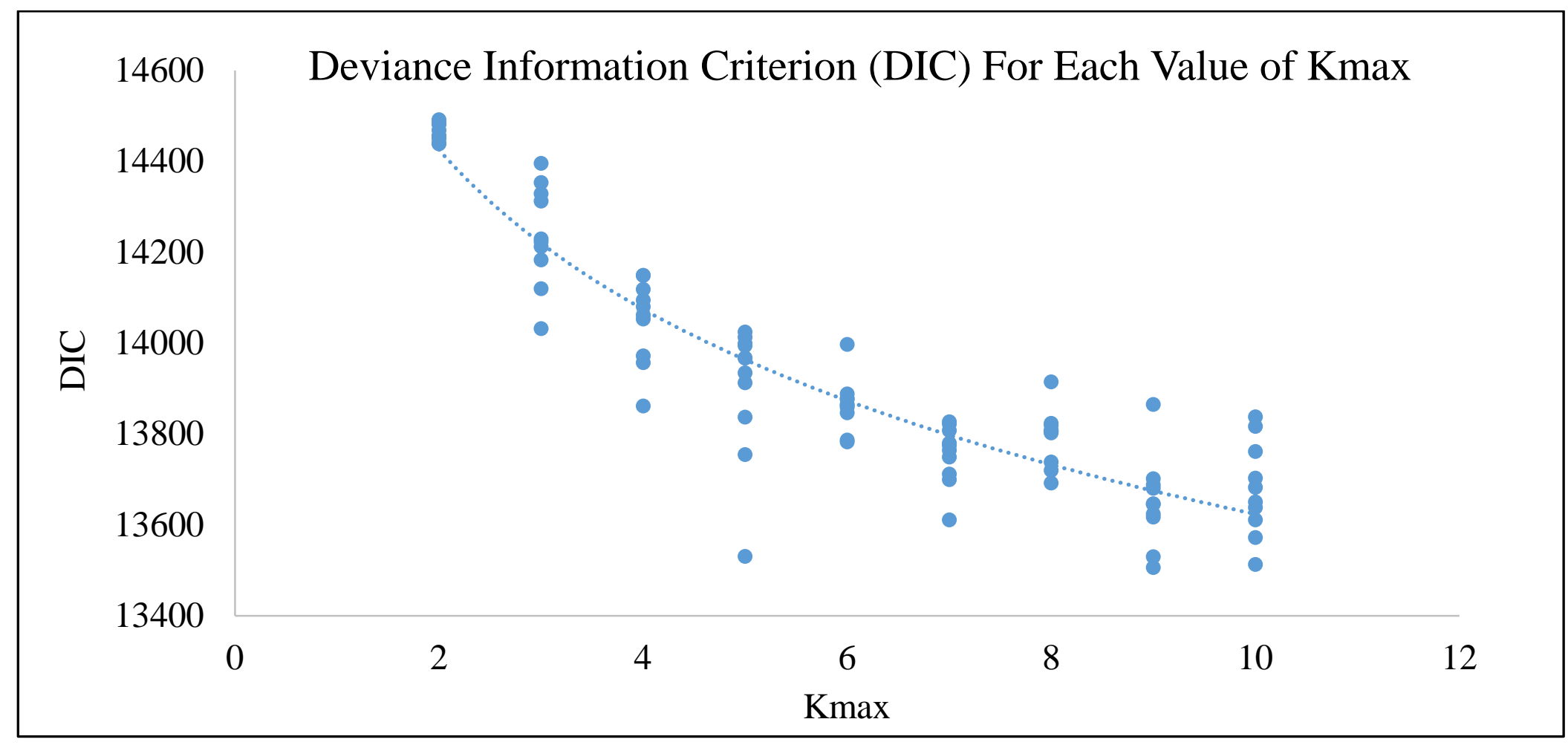

Figure 14 - The Deviance Information Criterion (DIC) for each value of the maximum number of populations (Kmax) as outputted by TESS v2.3 (Chen et al. 2007 , Durand et al. 2009) for 280 West Virginia bobcats genotyped at nine microsatellite loci.

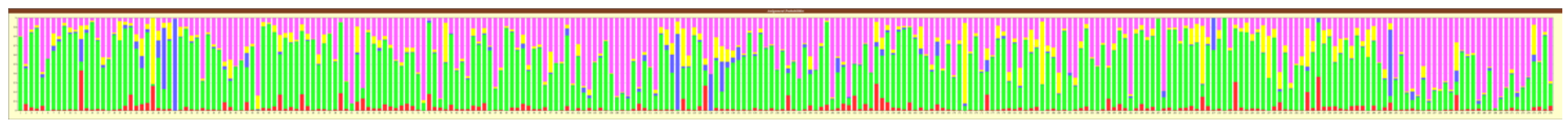

Figure 15 - Graphical output of population assignment probabilities for a second iteration of TESS v2.3 (Chen et al. 2007, Durand et al. 2009) for 280 West Virginia bobcats genotyped at nine microsatellite loci, when Kmax $=5$. 
The allelic richness (A) documented for each microsatellite locus of the single West Virginia bobcat population ranged from 9 - 15, with a mean of 12.44 (Table 16). Across all microsatellite loci, $\mathrm{H}_{\mathrm{O}}$ ranged between $0.645-0.845$, with a mean of 0.744 , in contrast to $\mathrm{H}_{\mathrm{E}}$ which ranged between $0.602-0.834$, with a mean of 0.729 . Out of the nine microsatellite loci, four (FCA008, FCA77, 6HDZ057, and 6HDZ700) were found to be out of HWE, after the Bonferroni correction of CERVUS $(\alpha=0.0056)$. Three of the four loci had an excess of heterozygotes, while the other locus had a homozygote excess.

Table 16 - Allelic richness (A), observed heterozygosity $\left(\mathrm{H}_{\mathrm{O}}\right)$, expected heterozygosity $\left(\mathrm{H}_{\mathrm{E}}\right)$, and p-value for test of Hardy-Weinberg Equilibrium deviation significance $\left(\mathrm{HWE}_{\mathrm{p}}\right)$ for each examined microsatellite locus of a 280 bobcat dataset collected in West Virginia between 2014 - 2015. HWE values with an asterisk indicate those that were found to be significantly out of Hardy-Weinberg Equilibrium by CERVUS v.3.0 after using a Bonferroni correction $(\alpha=0.0056)$.

\begin{tabular}{ccccc}
\hline Locus & A & Ho & HE & HWE $_{\boldsymbol{p}}$ \\
\hline FCA90 & 9 & 0.845 & 0.834 & 0.0742 \\
FCA23 & 10 & 0.716 & 0.772 & 0.2758 \\
FCA26 & 13 & 0.645 & 0.680 & 0.3649 \\
6HDZ057 & 11 & 0.702 & 0.602 & $2.683 \times 10^{-11 *}$ \\
FCA008 & 15 & 0.849 & 0.790 & $0.0011^{*}$ \\
FCA77 & 15 & 0.706 & 0.814 & $1.194 \times 10^{-4 *}$ \\
FCA43 & 15 & 0.736 & 0.672 & 0.0148 \\
6HDZ700 & 11 & 0.736 & 0.674 & $1.569 \times 10^{-4 *}$ \\
FCA45 & 13 & 0.777 & 0.725 & 0.1519 \\
\hline
\end{tabular}

As calculated by LDNe using a lowest allele frequency of 0.02 , the effective population size $\left(\mathrm{N}_{\mathrm{e}}\right)$ of West Virginia’s bobcat population was 1,448.7, with a 95\% parametric confidence interval of $535.6-\infty$, and a jackknifed $95 \%$ confidence interval across all loci of $426.8-\infty$. Both the Sign Test $(\mathrm{p}=0.0277)$ and the Wilcoxon one-tailed test for heterozygote deficiency $(\mathrm{p}=$ 0.0098) of BOTTLENECK produced evidence of a recent, statistically significant $(\alpha=0.05)$ population bottleneck in West Virginia's bobcats. Seven of the nine microsatellite loci examined had a heterozygote deficiency, as compared to the expected heterozygosity estimated by the two- 
phase model and the predicted mutation-drift equilibrium. This contemporary reduction in heterozygosity was attributed to a rapid reduction in the effective population size that occurred in recent history.

\section{Discussion}

As a whole, the bobcat population of West Virginia appears to be fairly genetically diverse, based on the microsatellite loci examined in this study. With an average of 12.44 alleles per locus and a mean $\mathrm{Ho}_{\mathrm{O}}$ of 0.744 , these values are similar to what was documented by Millions and Swanson (2007) in bobcats of Michigan, and Anderson et al. (2015) in the regional study of Ohio, Kentucky, Pennsylvania, and West Virginia. The analyses of STRUCTURE, the PCA, and TESS failed to find any significant population genetic structure in West Virginia. The lack of detectable population structure indicates that gene flow is occurring at the statewide scale. While the scale of West Virginia may be large, genetic panmixia in bobcats has been documented across a much larger region, including most of the western United States (Croteau et al. 2012, Reding et al. 2012). Unlike what was found in California (Riley et al. 2006, Lee et al. 2012, Ruell et al. 2012, Serieys et al. 2015), the major interstate highways of West Virginia do not appear to be a significant barrier to bobcat gene flow. The same conclusion was also documented in Michigan (Millions and Swanson 2007).

An unexpected result of this study was the finding that of the nine microsatellite loci examined, four significantly deviated from what was expected by HWE. Of the four loci out of HWE, three were due to a heterozygote excess (6HDZ057, FCA77, and 6HDZ700), while FCA77 was out of HWE due to a heterozygote deficiency. Heterozygote excess may be a result of the significant population bottleneck that was also identified by the program BOTTLENECK. 
When $\mathrm{N}_{\mathrm{e}}$ for a population is reduced by a bottleneck event, an artificial heterozygote excess is often created because rare alleles become lost faster than overall heterozygosity (Luikart and Cornuet 1998). Rarer alleles only have a small impact on heterozygosity and allelic richness can be greatly reduced without significantly impacting heterozygosity (Allendorf 1986). Thus, the apparent heterozygote excess may be an artifact of the historic pre-bottleneck population, instead of an actual overabundance of heterozygotes. This heterozygote excess is likely an additive factor causing three of the tested loci to be out of HWE, but it is not the only probable cause. Both male and female juvenile bobcats disperse; however, males usually disperse over greater distances (Janečka et al. 2007, Croteau et al. 2010), creating a sex-biased dispersal that has been found to disrupt expected values of heterozygosity and may lead to deviation from HWE (Tucker et al. 2017). Also, bobcats have overlapping generations, a violation of HWE assumptions, which can also cause significant deviations between predicted and observed genotype frequencies (Waples 2015).

While the complete range for the $95 \%$ confidence interval of $\mathrm{N}_{\mathrm{e}}$ could not be calculated for West Virginia's bobcat population, the lowest possible value of any range was an $N_{e}$ of 426.8. The failure to establish an upper boundary for the 95\% confidence interval by LDNe is usually a result of analyzing a subset of individuals from a census population that is also quite large (Gilbert and Whitlock 2015). Even the minimum possible value is robust enough that, should panmixia continue, the bobcat population of West Virginia meets the recommendations of the 50/500 rule introduced by Franklin (1980), where a minimum $\mathrm{N}_{\mathrm{e}}$ of roughly 500 is required for a population to maintain its evolutionary potential. However, recent developments in the field of population genetics have brought the validity of the 50/500 rule into question, and Frankham et al. (2014) suggested the minimum $\mathrm{N}_{\mathrm{e}}$ needed to maintain the evolutionary potential of a 
population was $\mathrm{N}_{\mathrm{e}} \geq 1,000$. As the bobcat populations of Ohio and Maryland continue to recover, and bobcat populations increase in other nearby states (Roberts and Crimmins 2010), gene flow entering West Virginia from these outside areas is expected to increase $\mathrm{N}_{\mathrm{e}}$. However, this will only occur if the current level of landscape connectivity is maintained to ensure bobcats can move from one locality to another without resistance caused by anthropogenic disturbances.

The detection of genetic evidence for the occurrence of a recent bottleneck in the West Virginia bobcat population is consistent with expectations based on historic harvest records. Bounties were paid by the West Virginia Division of Natural Resources to successful bobcat hunters and trappers until the program was ended in 1961 (personal communication: Rich Rogers, WVDNR). In the roughly seven years leading up to the removal of the bounty program, a total of 10 bobcats harvested in the state were sold to fur buyers every year, down from the average of 400 per year three decades earlier. After the establishment of a four month trapping season, bobcat numbers have rebounded significantly since the 1970s; however, West Virginia's bobcats still bear genetic evidence of this historic bottleneck.

\section{Management Implications}

This research failed to find evidence of genetic population structure in the bobcats of West Virginia. Thus, statewide gene flow, or panmixia is thought to be occurring across the landscape. Potential barriers, such as interstate highways, or major rivers are not having a noticeable impact on bobcat gene flow statewide. Due to the lack of genetic structuring, the bobcats of West Virginia may continue to be managed as a single, contiguously distributed population. Also, the effective population size was found to be sufficient to conserve the evolutionary potential of the population against genetic drift, as long as current levels of 
connectivity are maintained. While West Virginia's bobcats were found to possess the genetic signatures of a recent bottleneck event, their allelic richness and levels of observed heterozygosity remain similar to populations in adjacent locations. To best conserve genetic diversity, management actions should aim to at least maintain or assure no loss of connectivity for this species across the regional landscape.

\section{Acknowledgements}

We thank the hunters and trappers of West Virginia for donating the bobcat carcasses from which spleen samples were collected and the WVDNR employees that facilitated this collection. We also thank the WVDNR personnel from District 2 that assisted in the bobcat spleen DNA extractions, as well as Stephanie Landry from West Virginia University. We thank Avery Owers for completing much of the laboratory work necessary for this chapter. Finally, we thank Darren Wood of WVU for sharing his expertise with TESS. Financial support for this dissertation was provided by the West Virginia Division of Natural Resources through a grant with the U.S. Fish and Wildlife Service Wildlife and Sport Fish Restoration Program (W-48-R) and matching state funds. 


\section{References}

Allendorf, F. W. 1986. Genetic Drift and the Loss of Alleles Versus Heterozygosity. Zoo Biology 5:181-190.

Anderson, C. S., S. Prange, and H. L. Gibbs. 2015. Origin and Genetic Structure of a Recovering Bobcat (Lynx rufus) Population. Canadian Journal of Zoology 93:889-899.

Chen, C., E. Durand, F. Forbes, and O. François. 2007. Bayesian Clustering Algorithms Ascertaining Spatial Population Structure: a New Computer Program and a Comparison Study. Molecular Ecology Notes 7:747-756.

Croteau, E. K., E. J. Heist, and C. K. Nielsen. 2010. Fine-scale Population Structure and SexBiased Dispersal in Bobcats (Lynx rufus) from Southern Illinois. Canadian Journal of Zoology 88:536-545.

Croteau, E. K., E. J. Heist, C. K. Nielsen, J. R. Hutchinson, and E. C. Hellgren. 2012. Microsatellites and Mitochondrial DNA Reveal Regional Population Structure in Bobcats (Lynx rufus) of North America. Conservation Genetics 13:1637-1651.

Durand, E., F. Jay, O. E. Gaggiotti, and O. François. 2009. Spatial Inference of Admixture Proportions and Secondary Contact Zones. Molecular Biology and Evolution 26:19631973.

Earl, D. A., and B. M. vonHoldt. 2012. STRUCTURE HARVESTER: A Website and Program for Visualizing STRUCTURE Output and Implementing the Evanno Method. Conservation Genetics Resources 4:359-361.

Falush, D., M. Stephens, and J. K. Pritchard. 2007. Inference of population structure using multilocus genotype data: dominant markers and null alleles. Molecular Ecology Notes 7:574-578.

Falush D., M. Stephens, J. K. Pritchard. 2003. Inference of Population Structure Using Multilocus Genotype Data: Linked Loci and Correlated Allele Frequencies. Genetics 164:1567-1587.

Frankham, R., C. J. A. Bradshaw, and B. W. Brook. 2014. Genetics in Conservation Management: Revised Recommendations for the 50/500 rules, Red List criteria and Population Viability Analyses. Biological Conservation 170:56-63.

Franklin, I. R. 1980. Evolutionary change in small populations. Sunderland, Massachusetts, Sinauer Associates, U.S.A. pp. 135-149.

Gilbert, K. J., and M. C. Whitlock. 2015. Evaluating Methods for Estimating Local Effective Population Size with and without Migration. Evolution 69:2154-2166. 
Holderegger, R., and M. Di Giulio. 2010. The Genetic Effects of Roads: A Review of Empirical Evidence. Basic and Applied Ecology 11:522-531.

Jakobsson, M., and N. A. Rosenberg. 2007. CLUMPP: A Cluster Matching and Permutation Program for Dealing with Label Switching and Multimodality in Analysis of Population Structure. Bioinformatics 23:1801-1806.

Janečka, J. E., T. L. Blankenship, D. H. Hirth, C. William Kilpatrick, M. E. Tewes, and L. I. Grassman. 2007. Evidence for Male-biased Dispersal in Bobcats Lynx Rufus Using Relatedness Analysis. Wildlife Biology 13:38-47.

Kalinowski, S. T., M. L. Taper, and T. C. Marshall. 2007. Revising How the Computer Program Cervus Accommodates Genotyping Error Increases Success in Paternity Assignment. Molecular Ecology 16:1099-1106.

Lacy, R. C. 1997. Importance of Genetic Variation to the Viability of Mammalian Populations. Journal of Mammalogy 78:320-335.

Landry, S. M. 2017. Bobcat Population Ecology in West Virginia. M.S. Thesis. West Virginia University. pp. 1-179.

Lee, J. S., E. W. Ruell, E. E. Boydston, L. M. Lyren, R. S. Alonso, J. L. Troyer, K. R. Crooks, and S. VandeWOUDE. 2012. Gene Flow and Pathogen Transmission Among Bobcats (Lynx rufus) in a fragmented urban landscape. Molecular Ecology 21:1617-1631.

Luikart, G., and J.-M. Cornuet. 1998. Empirical Evaluation of a Test for Identifying Recently Bottlenecked Populations from Allele Frequency Data. Conservation Biology 12:228237.

Menotti-Raymond, M. A., and S. J. O’Brien. 1995. Evolutionary Conservation of Ten Microsatellite Loci in Four Species of Felidae. Journal of Heredity 86:319-322.

Menotti-Raymond, M., V. A. David, L. A. Lyons, A. A. Schäffer, J. F. Tomlin, M. K. Hutton, and S. J. O’Brien. 1999. A Genetic Linkage Map of Microsatellites in the Domestic Cat (Felis catus). Genomics 57:9-23.

Menotti-Raymond, M., V. A. David, J. C. Stephens, L. A. Lyons, and S. J. O’Brien. 1997. Genetic Individualization of Domestic Cats Using Feline STR Loci for Forensic Applications. Journal of Forensic Sciences 42:1039-1051.

Millions, D. G., and B. J. Swanson. 2007. Impact of Natural and Artificial Barriers to Dispersal on the Population Structure of Bobcats. Journal of Wildlife Management 71:96-102.

Moritz, C. 1994. Defining ‘Evolutionarily Significant Units’ for Conservation. Trends in Ecology \& Evolution 9:373-375. 
Peakall, R. and P.E. Smouse. 2012. GenAlEx 6.5: Genetic Analysis in Excel. Population Genetic Software for Teaching and Research-an Update. Bioinformatics 28:2537-2539.

Peakall, R. and P.E. Smouse. 2006. GenAlEx 6: genetic analysis in Excel. Population Genetic Software for Teaching and Research. Molecular Ecology Notes. 6:288-295.

Piry, S., G. Luikart, and J.-M. Cornuet. 1999. Computer Note. BOTTLENECK: A Computer Program for Detecting Recent Reductions in the Effective Size Using Allele Frequency Data. Journal of Heredity 90:502-503.

Pritchard J. K., Stephens M., Donnelly P. 2000. Inference of Population Structure Using Multilocus Genotype Data. Genetics. 155: 945-959.

Reding, D. M., A. M. Bronikowski, W. E. Johnson, and W. R. Clark. 2012. Pleistocene and Ecological Effects on Continental-scale Genetic Differentiation in the Bobcat (Lynx rufus). Molecular Ecology 21:3078-3093.

Reid, A. E. 2006. Spatial Genetic Structure of Four Bobcat Populations in the Southeastern US. M.S. Thesis. University of Georgia: pp 1-122.

Riley, S. P. D., J. P. Pollinger, R. M. Sauvajot, E. C. York, C. Bromley, T. K. Fuller, and R. K. Wayne. 2006. FAST-TRACK: A Southern California Freeway is a Physical and Social Barrier to Gene Flow in Carnivores. Molecular Ecology 15:1733-1741.

Roberts, N. M., and S. M. Crimmins. 2010. Bobcat Population Status and Management in North America: Evidence of Large-Scale Population Increase. Journal of Fish and Wildlife Management 1:169-174.

Ruell, E. W., S. P. D. Riley, M. R. Douglas, M. F. Antolin, J. R. Pollinger, J. A. Tracey, L. M. Lyren, E. E. Boydston, R. N. Fisher, and K. R. Crooks. 2012. Urban Habitat Fragmentation and Genetic Population Structure of Bobcats in Coastal Southern California. The American Midland Naturalist 168:265-280.

Serieys, L. E. K., A. Lea, J. P. Pollinger, S. P. D. Riley, and R. K. Wayne. 2015. Disease and Freeways Drive Genetic Change in Urban Bobcat Populations. Evolutionary Applications 8:75-92.

Tucker, J. M., F. W. Allendorf, R. L. Truex, and M. K. Schwartz. 2017. Sex-Biased Dispersal and Spatial Heterogeneity Affect Landscape Resistance to Gene Flow in Fisher. Ecosphere 8:e01839.

Van Oosterhout, C., W. F. D. Hutchinson, D. Wills, and P. Shipley. 2004. MICRO-CHECKER: Software for Identifying and Correcting Genotyping Errors in Microsatellite Data. Molecular Ecology Notes 4:535-538. 
Waples, R. S. 2015. Testing for Hardy-Weinberg Proportions: Have We Lost the Plot? Journal of Heredity 106:1-19.

Waples, R. S., and C. Do. 2008. ldne: A Program for Estimating Effective Population Size from Data on Linkage Disequilibrium. Molecular Ecology Resources 8:753-756.

Williamson, J. E., R. M. Huebinger, J. A. Sommer, E. E. Louis, and R. C. Barber. 2002. Development and Cross-species Amplification of 18 Microsatellite Markers in the Sumatran Tiger (Panthera tigris sumatrae). Molecular Ecology Notes 2:110-112. 


\title{
Chapter 6 - Prevalence and Molecular Characterization of Feline \\ Panleukopenia Virus (FPV) and Canine Parvovirus (CPV) in Bobcats of West Virginia $^{2}$
}

\begin{abstract}
Feline Panleukopenia Virus (FPV) and Canine Parvovirus (CPV), common pathogens of domestic cats (Felis catus) and domestic dogs (Canis familiaris), have been documented worldwide in numerous wildlife species. Data are lacking on local prevalence rates, as well as the potential impact these pathogens may be having on the bobcat (Lynx rufus). Spleen samples were collected from a total of 528 bobcats harvested in West Virginia during the 2014 - 2015 and 2015 - 2016 hunting and trapping seasons. PCR followed by DNA sequencing was used to determine which bobcats were infected with FPV or CPV at the time of harvest, as well as the viral type infecting each animal. Of the tested bobcats, $17.6 \%$, were found to be infected, of which 85.4\% were infected with CPV-2 / CPV-2a, 11.5\% were infected with CPV-2b, and 3.1\% were infected with FPV. A total of 96 sequences were analyzed since three bobcats were found to be simultaneously co-infected with two different viral types. A median-joining network revealed that the 96 collected DNA sequences consisted of 18 unique viral haplotypes. A spatial analysis identified a statistically significant hotspot of CPV / FPV infection in northwestern West Virginia, which was not found to be associated with an increased human population density.
\end{abstract}

\footnotetext{
2 This chapter was co-authored with Stephanie Landry while she was working on her M.S. degree at West Virginia University.
} 


\section{Introduction}

Feline panleukopenia virus (FPV) is a parvovirus with single-stranded DNA that belongs to the genus Parvovirus and has been recognized since the 1920s (Siegl et al. 1985, Horiuchi et al. 1996). Certain strands have displayed high rates of nucleotide change, allowing them to rapidly replicate and create new host variants (Decaro et al. 2008, Allison et al. 2013). Canine parvovirus (CPV) was discovered in the late 1970s (Decaro et al. 2010, Allison et al. 2013, Clegg et al. 2012) and is one of the best examples of viral emergence leading to an epidemic in a new host. It is a type of feline parvovirus that has been replicated in vivo in cats where it became a host variant after a cross-species transfer from the Feline Panleukopenia Virus (FPV) in felids to canids (Decaro et al. 2010, Allison et al. 2013, Stuetzer and Hartmann 2014). Canine Parvovirus was initially identified to have only one variation of the virus-CPV-2. Since its initial discovery in the 1970s, it now contains three different antigenic types of Parvoviruses: CPV-2a, CPV-2b (Schunck et al. 1995), and most recently CPV-2c that was detected in Italy in 2000 and is now found worldwide (Decaro et al. 2010). Since the 2000s, newer variants of CPV have adapted to infect feline species as well (Wasieri et al. 2009) and can co-infect an individual feline simultaneously with FPV (Decaro et al. 2008, Battilani et al. 2011, 2013). Recent taxonomic research has resulted in the reclassification of CPV and FPV into Carnivore protoparvovirus 1 (Cotmore et al. 2014), which includes Mink Enteritis Virus (MEV), and Raccoon Parvovirus (RaPV).

Both FPV and CPV cause acute disease in all members of Felidae (Demeter et al. 2010, Kruse et al. 2010, Foley et al. 2013). FPV infection can result in severe gastroenteritis (swelling and inflammation of the small intestines, anorexia, fever, pyrexia, diarrhea, vomiting, leukopenia, lymphopenia, lethargy, and weakness - with symptoms beginning one to four days 
post-infection and lasting up to two weeks (Berthier et al. 2000). The combination of these symptoms often leads to death of an infected animal—especially in kittens and juveniles (Kruse et al. 2010, Horiuchi et al. 1996, Schunck et al. 1995). Infected individuals shed viral particles into the environment in feces, nasal and ocular discharges, urine, saliva, and vomit for up to 22 days post-infection (Csiza et al. 1971). These viruses may remain highly infectious in the environment for up to a year (Waseiri et al. 2009, Foley et al. 2013). The viruses are typically transmitted through fecal ingestion (Schunck et al. 1995) or contact with contaminated individuals or objects (Wasieri et al. 2009). Parvoviruses may even move between hosts during predation or scavenging interactions between domestic and wild carnivores, such as bobcats (Allison et al. 2013).

Infections of FPV are common in domestic cats, often occurring in kittens at the time of weaning or under 6 months of age (Wasieri et al. 2009, Stuetzer and Hartmann 2014). Kittens, especially when stressed or co-infected with other pathogens, are subject to high mortality rates between 25 - 90\% in acute cases (Wasieri et al. 2009, Kruse et al. 2010). Feral cats (Felis catus) are carriers and common transmitters of FPV to non-domestic felid populations in heavily populated areas (Wasieri et al. 2009), such as mountain lions (Puma concolor) in California (Foley et al. 2013). Increased human densities may incidentally facilitate disease transmission to wild felids with the increasing overlap of non-domestic and domestic felid territories (Foley et al. 2013, Wasieri et al. 2009).

Among wildlife managers there is concern that the expansion of FPV and CPV (Carnivore protoparvovirus 1) poses a threat to numerous wildlife species. However, the prevalence of the virus and the impact to non-domestic populations are unknown and rarely investigated. Many scientists have encouraged an increase in host sampling to gather more 
information about how Carnivore protoparvovirus 1 is evolving and spreading (Allison et al. 2013). The West Virginia Division of Natural Resources (WVDNR) is concerned that Carnivore protoparvovirus 1 in West Virginia is spreading from domestic felids to wild bobcats. To begin evaluating the potential impact that these viruses are having on bobcat ecology in West Virginia, we tested samples from harvested animals to complete the following objectives: 1) investigate bobcat spleen samples for presence of parvovirus DNA and determine overall statewide prevalence rate; 2) determine type of parvovirus infecting each bobcat found to be positive; 3) determine if prevalence of bobcats with parvovirus infections is greater than would be expected by random chance in more urbanized counties of West Virginia; 4) investigate presence of potential geographical patterns in different types of parvoviruses found in West Virginia’s bobcats.

\section{Materials and Methods}

\section{Sample Collection}

During the 2014-2015 and the 2015-2016 hunting and trapping seasons (November February) the WVDNR collected voluntarily-donated bobcat carcasses from successful hunters and trappers. At the time of collection, the county from which the animal was harvested was recorded for further analyses. A total of 303 carcasses were collected during the 2014-2015 season and 225 were collected during the 2015—2016 season. A necropsy was completed on each bobcat carcass and entire spleens were removed for DNA analyses. Both bobcat carcasses and collected spleens were stored at $-20^{\circ} \mathrm{C}$ between procedures. 


\section{Parvovirus Detection}

Of the most common laboratory diagnostic tests for parvovirus infection, the polymerase chain reaction (PCR) was found by to be the most sensitive technique-as compared to the traditional methods of hemagglutination, immunochromatography, and virus isolation techniques (Desario and Decaro 2005). Traditional PCR of viral DNA was found to detect as little as $10^{3}$ Plaque Forming Units (PFU)/mL in fecal samples (Schunck et al. 1995). PCR has been successfully used to detect viral DNA in the gastrointestinal tract, spleen, liver, lungs, heart, bone marrow, mesenteric lymph node, tongue, or feces (Meurs et al. 2000, Haynes and Holloway 2012, Allison et al. 2013). Though PCR is the preferred method for identifying positively infected individuals, it only detects the presence of a current infection. It cannot detect if the individual was previously infected, nor can it accurately quantify the amount of infectious particles without substantial experimental efforts.

From each spleen sample collected during necropsy, a 10 - 15 mg subsample was removed and placed into a 1.5mL microcentrifuge tube. DNA extractions were completed using the ThermoFisher (Pittsburgh, PA) GeneJet Tissue and Cells DNA Extraction Kit. The manufacturer's suggested protocol was utilized throughout the extraction procedures, except for the exclusion of the 3-hour incubation. The only other modification was that $30 \mu \mathrm{L}$ of $1 \mathrm{M}$ DTT was also added to the recommended reagents to assist in the denaturation of splenic proteins. Once completed, the DNA extracts were normalized to a $10 \mathrm{ng} / \mu \mathrm{L}$ concentration using a ThermoFisher NanoDrop Lite spectrophotometer. To quickly screen out samples that were negative for parvovirus DNA, the VP1 and VP2 primer pair (Schunck et al. 1995) was used in an attempt to amplify parvovirus DNA from each spleen extract. This primer set was purposefully 
selected since it has lower specificity than other available primer sets and a smaller product size so it could be used to quickly screen and eliminate negative samples.

PCR reactions were completed using the following reagents and concentrations: $5 \mu \mathrm{L}$ of the 2x Qiagen Multiplexing Master Mix (Germantown, MD), 1 $\mu$ mol of the VP1 / VP2 primer set, and a minimum of 40ng of extracted DNA template (this template contained both viral and bobcat DNA so the actual amount of viral DNA used in each PCR is unknown). Thermal cycling conditions were modified from Schunck et al. (1995). A $94^{\circ} \mathrm{C}$ hot-start for 5 minutes was followed by 10 cycles of $95^{\circ} \mathrm{C}$ for $30 \mathrm{~s}, 60^{\circ} \mathrm{C}$ for 30 s, and $72^{\circ} \mathrm{C}$ for 60 s, with the annealing temperature being reduced by $1^{\circ} \mathrm{C}$ every cycle. This was followed by 25 additional cycles of $94^{\circ} \mathrm{C}$ for 30 s, $55^{\circ} \mathrm{C}$ for 90 s, and $72^{\circ} \mathrm{C}$ for 60 s, with a final extension of 30 minutes at $72^{\circ} \mathrm{C}$. PCR products were then resolved on a $1 \%$ agarose gel at $120 \mathrm{v}$ for 30 minutes. The presence of a band at roughly 200nt indicated a positive result for parvovirus DNA in the bobcat spleen extract.

Spleen samples determined to be positive for parvovirus DNA using the VP1/VP2 primer set were then amplified a second time via PCR. This second PCR reaction was used to reduce the possibility that contamination resulting from the first PCR would cause false positive results. The second amplicon was described by Buonavoglia et al. (2001) as 555for / 555rev and was designed specifically to sequence a variable region of the VP2 gene that allows for differentiation between the variants of Carnivore protoparvovirus 1: FPV, CPV (and types), MEV, and RaPV (Cotmore et al. 2014). These PCRs were completed in 20 $\mu \mathrm{L}$ reaction sizes with $10 \mu \mathrm{L}$ of 2x Qiagen Multiplexing Master Mix, $1 \mu \mathrm{mol}$ of the 555for / 555rev primer mix at least 150ng of total combined viral DNA and bobcat extract. The thermal cycling protocol was as follows: a $94^{\circ} \mathrm{C}$ hot-start for 10 minutes, followed by 43 total cycles of $94^{\circ} \mathrm{C}$ for $30 \mathrm{~s}, 55^{\circ} \mathrm{C}$ for 30 s, $72^{\circ} \mathrm{C}$ for 45 s, followed by a 10 minute final extension at $72^{\circ} \mathrm{C}$. Half $(10 \mu \mathrm{L})$ of each PCR 
product was then run on a $1 \%$ agarose gel at $120 \mathrm{v}$ for 30 minutes, with a positive result indicated by a band at roughly 550nt. Samples that successfully amplified DNA with both primer sets were then prepared for DNA sequencing using the remaining $10 \mu \mathrm{L}$ from this previous PCR and a QIAquick 96-well plate PCR Purification Kit on each sample to remove primers and contaminants. These PCR products were then Sanger sequenced using the Applied Biosystems (ABI) BigDye V.3 reaction chemistry for the sequencing reaction followed by resolution on an ABI 3130XL genetic analyzer. Samples were only considered to be positive for Carnivore protoparvovirus 1 if they produced a DNA sequence able to be used in downstream analyses.

\section{Phylogenetic Analyses}

Viral DNA sequences were aligned using the ClustalW application of the program BioEdit v.7.2.6 (Hall 1999), combined with manual manipulation. Known viral sequences of CPV2a (HQ589341.1), CPV2b (HQ589342.1), CPV2c (HQ450028.1), FPV (EU221280.1), and MEV (D00765.1), with these accession numbers, were acquired from the National Center for Biotechnology Information’s GenBank and were aligned to the sequences derived from this study. The viral type of sequenced samples was determined manually using the type-specific mutations described by Buonavoglia et al. (2001) at positions 4062 and 4449 (amino acids 426 and 555, respectively) of the VP2 gene, similar to the method described in Ohneiser et al. (2015). After each viral type was identified a median-joining haplotype network (Bandelt et al. 1999) was constructed using these sequences. NETWORK 5.0.0.3 (fluxus-engineering.com) was used with its default parameters for median-joining networks. Geographical distribution of the generated haplotype network was then investigated using the ecological regions of West Virginia as described by Uhlig and Wilson (1952) (appendix Figure 1). 


\section{Geospatial Analyses}

Geospatial analyses were completed in ArcMap v.10.4.1 (ESRI) to investigate any spatial patterns for the prevalence of parvoviruses or for certain viral types. From an initial West Virginia county shapefile, centroid points were created to be used for continued analyses. The Incremental Spatial Autocorrelation tool was then used to find the optimal spatial band size for each analysis to be completed. Next, the Getis-Ord Gi Hotspot analysis (Getis and Ord 1992, Ord and Getis 1995) was used to statistically determine if any patterns were present for the spatial arrangement of Parvovirus prevalence or viral types. For conceptualization of spatial relationships, the Zone of Indifference was used and the value of the first major peak from the Incremental Spatial Autocorrelation test was used as the distance band input. In addition, the False Discovery Rate correction was used to reduce the likelihood of finding erroneous relationships. The Inverse-Distance Weighted (IDW) tool was used to create an interpolated surface of the z-scores from statistically significant Getis-Ord Gi Hotspot analysis. For this analysis, z-scores with a percentile weight greater $90 \%$ or less than $10 \%$ were considered statistically significant indicators of hotspots or coldspots, respectively. Finally, to test the hypothesis that viral prevalence and landscape distribution are associated with human population density, a Pearson's correlation analysis was conducted on the Carnivore protoparvovirus 1 prevalence data and the 2016 population density estimates for each county of West Virginia (United States Census Bureau 2016).

\section{Results}

Of the original 528 spleen samples tested for the presence of Carnivore protoparvovirus 1,17.6\% $(\mathrm{n}=93)$, were positive. While viral DNA was successfully amplified from 93 samples, 
three were found to be co-infected with two different sequences of Carnivore protoparvovirus 1, bringing the total number of examined sequences to 96 . As such, a total of 96 viral DNA sequences were isolated and used for further analyses. Of these sequences, $85.4 \%(n=82)$ were determined to be CPV-2 / CPV-2a, 11.5\% $(\mathrm{n}=11)$ were CPV-2b, and 3.1\% $(\mathrm{n}=3)$ were identified as FPV. Due to the length of the DNA sequence used for this analysis, a differentiation between CPV-2 / CPV-2a could not be completed. Variant CPV-2c was not detected amongst these samples. Of the 46 counties in West Virginia tested for the presence of Carnivore protoparvovirus 1, at least one infected bobcat was documented in each of 36 counties, indicating a statewide distribution of the virus. Prevalence of Carnivore protoparvovirus 1 appears to be greatest in north-central West Virginia, as compared to the southern counties of the state, where prevalence is lower (Figure 16; appendix Table 2). 


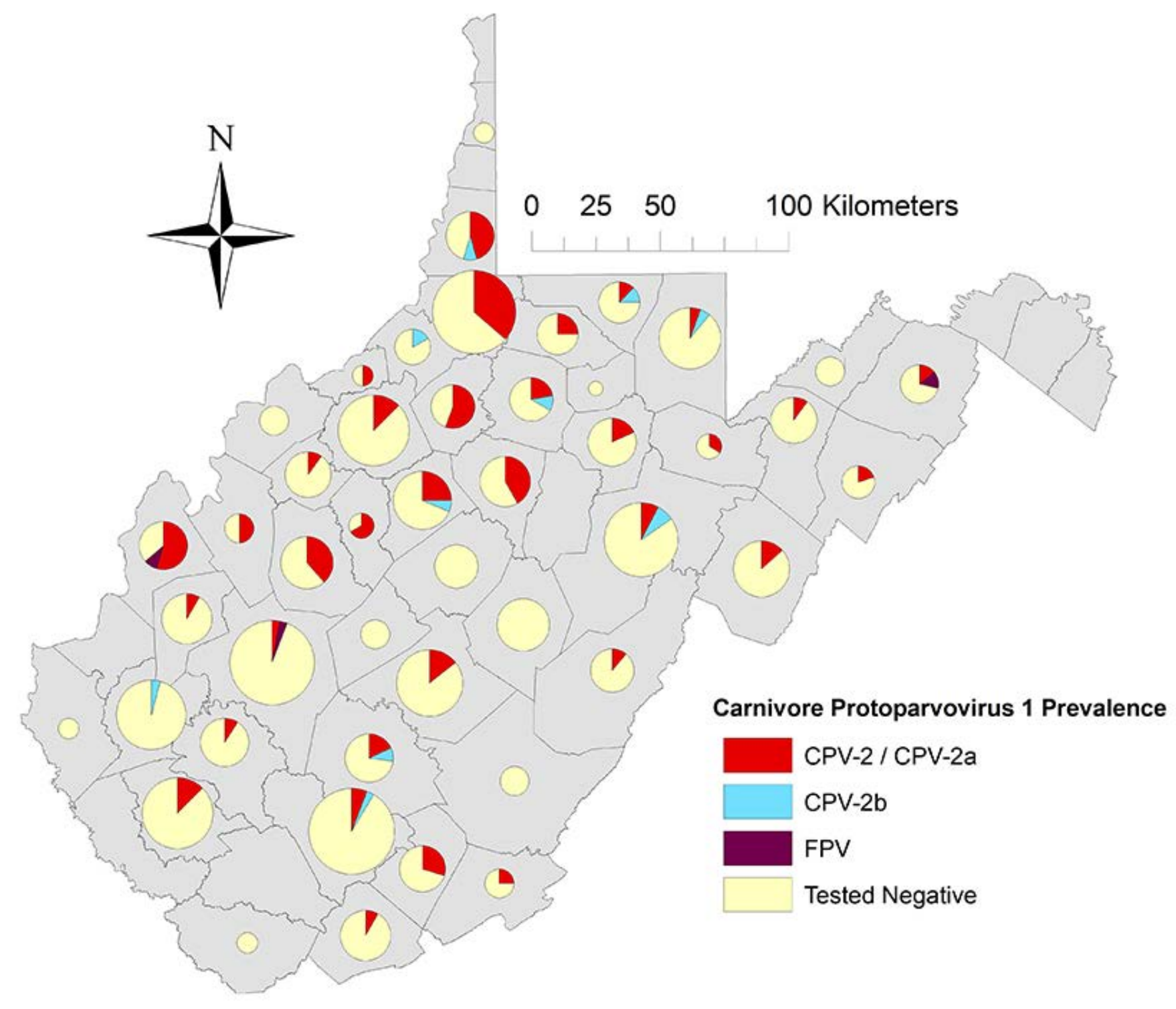

Figure 16 - Map of West Virginia detailing the prevalence of Carnivore protoparvovirus 1 in each county from which bobcat carcasses were collected during 2014 - 2016. The size of the pie chart in each county is indicative of the number of samples collected from each county and the color of the pie slice indicates the prevalence rate of each viral type detected. The detailed information used to create this map can be found on Table 1 of the Appendix. 


\section{Phylogenetic Results}

Of the 96 Carnivore protoparvovirus 1 sequences generated in this study, a total of 18 different haplotypes were identified: $75.3 \%$ were assigned to the four prevalent haplotypes, while the remaining 12 haplotypes were comprised of 1 - 4 sequences (Figure 17, appendix Table 3). Also, 22 of the West Virginia sequences derived in this study were found to have a substitution of $A \rightarrow G$ at VP2 position 4286, which was not found in any reference Carnivore protoparvovirus 1 sequences examined on the National Center for Biotechnology Information (NCBI) Genbank database. While other local variations were found in the West Virginia haplotypes, none were as significant as this polymorphism, which was present in nearly $25 \%$ of the entire dataset. 


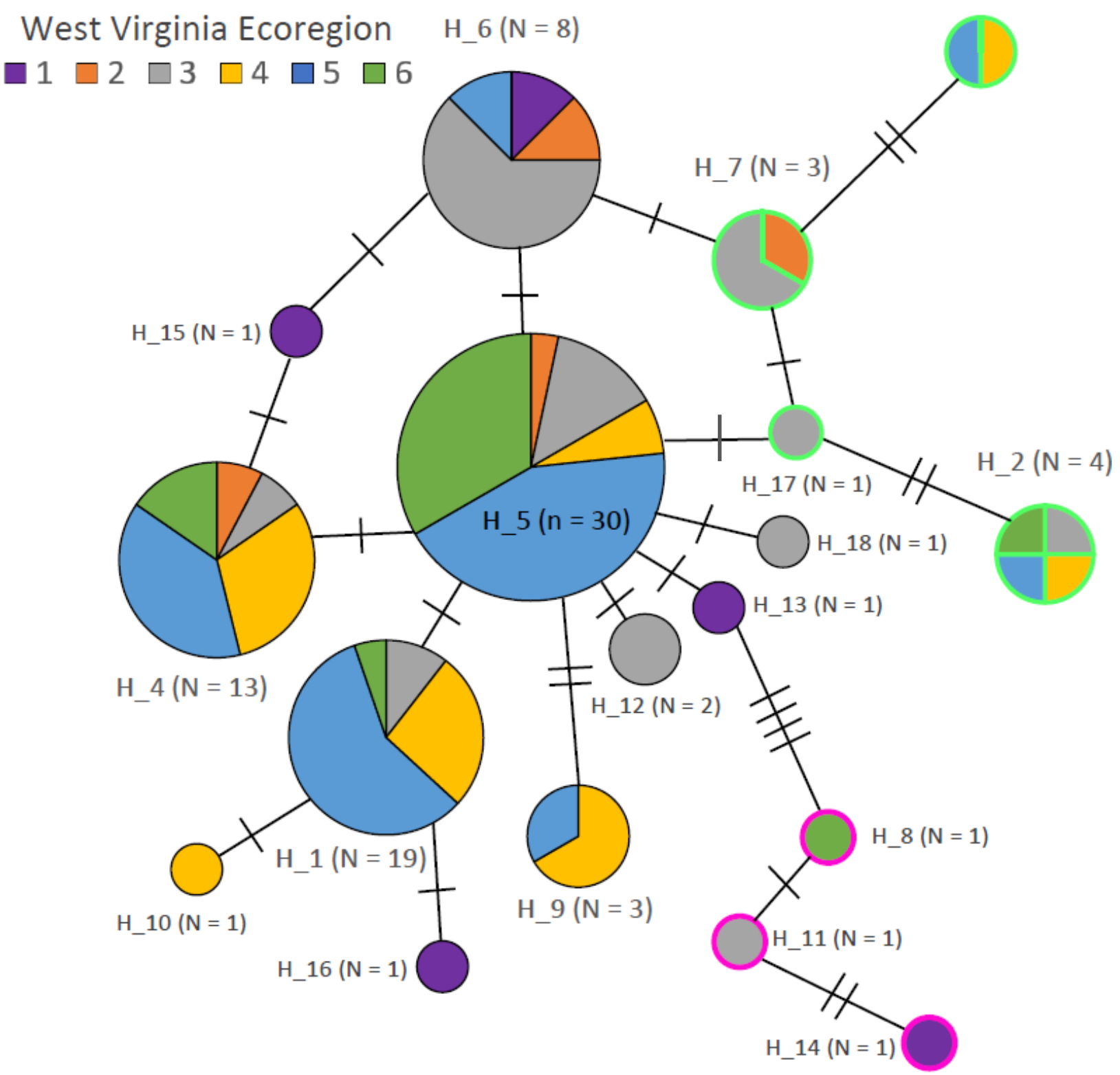

Figure 17 - Median-joining haplotype network as constructed using the program NETWORK 5.0.0.3 for Carnivore protoparvovirus 1 sequences amplified from bobcat spleens collected in West Virginia between 2014 - 2016. The size of each node is dependent on the number of samples that were found for each haplotype. Hashmarks on each linkage indicate the number of polymorphisms between the related haplotypes. Color of each pie slice is indicative of the ecological region (appendix Figure 1) from which each sample was collected. Outline color of the nodes indicates the viral typing of that particular haplotype. Nodes outlined in pink were determined to be FPV, those outlined in green were CPV-2b, and those outlined in black were CPV-2 / CPV-2a. 


\section{Geospatial Analyses}

Two spatial analyses were conducted on this viral sequence dataset to investigate the presence of spatial patterns, one for CPV2b and one for all Carnivore protoparvovirus 1 detections. Due to the low sample size of detected FPV infections $(\mathrm{n}=3)$, no further analyses were conducted on this viral subset. In contrast, no individual spatial analysis for CPV2 / CPV2a was completed since it made up $86 \%$ of the total sample and would be heavily correlated to a spatial analysis of all detected types of Carnivore protoparvovirus 1. The Getis-Ord Gi Hotspot analysis carried out on CPV2b did not reveal any hotspots across West Virginia. Thus, geospatial distribution of CPV2b does not appear to follow a particular spatial pattern. However, the same analysis conducted on all positive samples found a statistically significant hotspot of Carnivore protoparvovirus 1, centered around Doddridge, Tyler, and Wetzel Counties (Figure 18). Finally, correlation analysis between human population density and Carnivore protoparvovirus 1 prevalence produced an $\mathrm{R}^{2}$ value of 0.03 (appendix Figure 2), providing no evidence to support the claim that viral prevalence in wild bobcats is associated with human population density. 


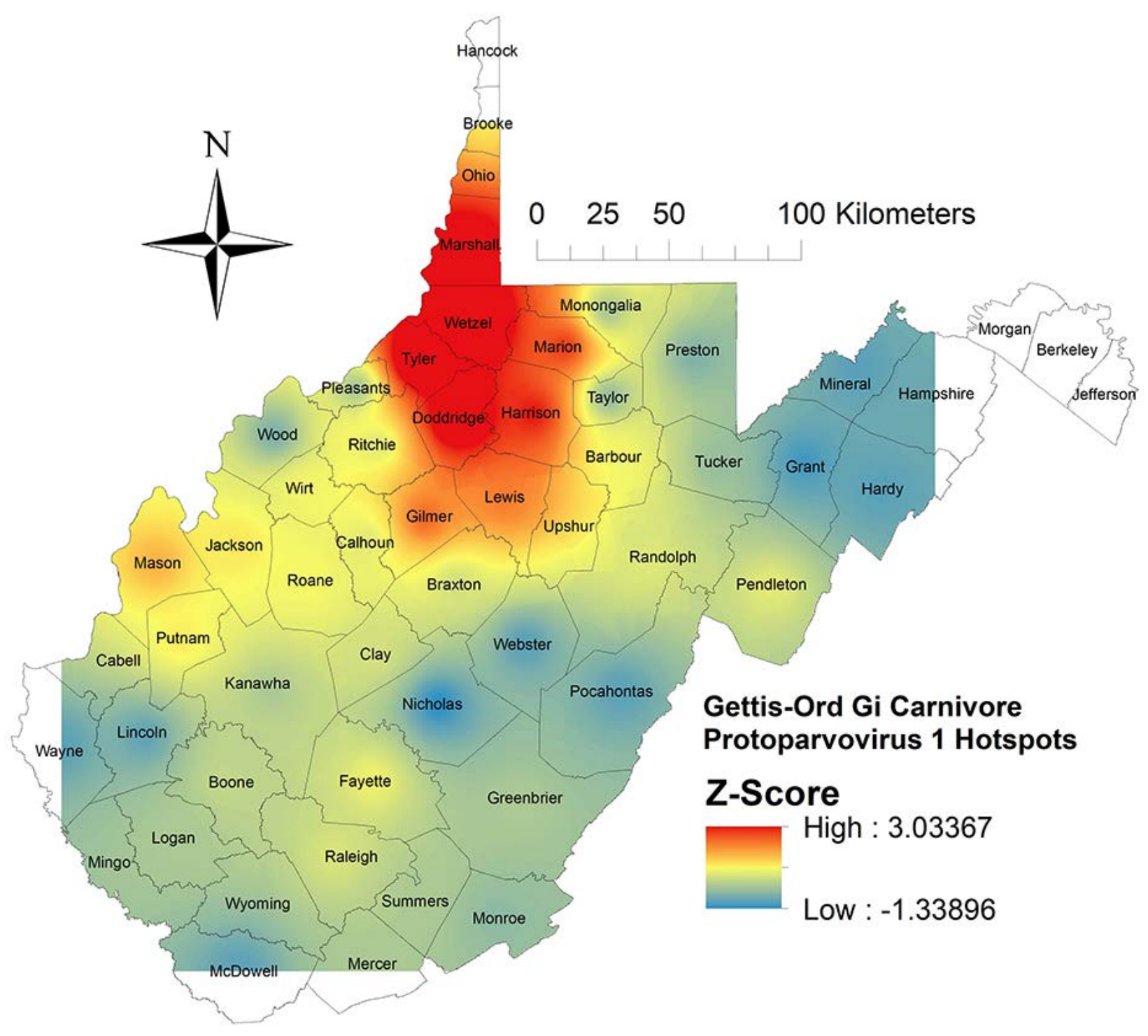

Figure 18 - Inverse Distance Weighted (IDW) interpolated map of Z-scores from the Getis-Ord Gi Hotspot Analysis of Carnivore protoparvovirus 1 prevalence rates for each county of West Virginia. Viral DNA sequences were amplified from bobcat spleens collected from 2014 - 2016. Locations in red indicate areas where prevalence of Carnivore protoparvovirus 1 is much greater than would be expected in an even distribution and locations in blue indicate areas where prevalence of Carnivore protoparvovirus 1 is less than what would be expected in an even distribution. Locations in yellow are those that have no statistically significant relationships. 


\section{Discussion}

The $17.6 \%$ Carnivore protoparvovirus 1 prevalence rate is similar to other studies on wild bobcats. Carver et al. (2016) found highly variable prevalence rates when using ELISA to screen for FPV / CPV in bobcats of California ( 10\%), Colorado ( 20\%), and Florida ( 40\%). While the virus has now been documented statewide, the true impact it is having on West Virginia’s bobcat population is unknown.

CPV-2 / CPV-2a was determined by a wide margin to be the most common type of Carnivore protoparvovirus 1 in West Virginia’s bobcats, comprising 86.5\% of total viruses sequenced. In contrast, this study failed to detect CPV-2c. These findings are similar to a recent worldwide study by Zhou et al. (2017) which found that CPV-2 / CPV-2a and CPV-2b were more common than CPV-2c in North America. While the original purpose of this study was to identify the prevalence of FPV in West Virginia’s bobcat population, FPV infection was rare, with an overall prevalence of only $0.57 \%$ from the total population and roughly $3 \%$ of the total

Carnivore protoparvovirus 1 sequences generated. Throughout the world, CPV variants are more commonly found in felids than the original FPV, which is disappearing rapidly, due to the suspected more virulent capsid antigens of CPV (Ikeda et al. 2000, Hoelzer et al. 2008). This trend of CPV replacing FPV was also documented in domestic felids of Vietnam and Taiwan (Ikeda et al. 2000) the United Kingdom (Clegg et al. 2012), and many species of wildlife in the United States (Allison et al. 2013).

Hotspot analysis identified a statistically-significant region with greater than expected prevalence of Carnivore protoparvorvirus 1. It was originally hypothesized that, if a viral hotspot were present, it would be centered on areas of increased human population due to the possible cross-species transfer between wild and domestic felids (Wasieri et al. 2009, Foley et al. 
2013). However, the counties over which this hotspot is situated have lower human population densities than most of the other counties in West Virginia (appendix Figure 2). Again, the correlation analysis between the human population density of each county of West Virginia and the prevalence rate of Carnivore protoparvovirus 1, found no relationship between these factors. Smaller sample sizes in some counties may be confounding this analysis; however, it is clear that there is little evidence for a relationship between human population density and Carnivore protoparvovirus 1 prevalence.

While it cannot be directly evaluated by this research, the location of this hotspot is not likely to be solely due to wildlife-domestic animal interactions. A recent study by Carver et al. (2016) investigated the cross-species transmission of six different pathogens in the domestic cats, mountain lions, and bobcats of California, Colorado, and Florida. The final conclusion of their spatial analysis was that exposure of wild felids to pathogens was often negatively associated with the wildland-urban interface. The authors hypothesized that cross-species disease transmission events are rare, and that it is more likely that these pathogens replicate in the new host species after introduction. A similar relationship may exist between the domestic and wild felids of West Virginia; however, future research will be needed to evaluate the causes behind this Carnivore protoparvovirus 1 hotspot.

While the most common haplotypes in this analysis (H_1, H_4, H_5 and H_6) made up over $75 \%$ of the generated viral sequences, there were a number of unique variants that were only sequenced a few times. A total of 27 polymorphic sites were identified in the viral sequences of this study, which is similar to what has been found in other localities, such as New Zealand (Ohneiser et al. 2015). When examining the haplotype map distribution geographically, three of the largest haplotype clusters $\left(\mathrm{H} \_1, \mathrm{H} \_4\right.$, and $\left.\mathrm{H} \_5\right)$ appear to be most closely associated 
with ecoregions 4 and 5, which are located within the newly identified Carnivore protoparvovirus 1 hotspot. When looking across the landscape, no single haplotype was found in all six ecological regions, which suggests that local viral evolution may be the driving force behind viral diversity instead of the mixing of viral types between ecological regions. As such, the limited viral diversity of ecoregions 1 and 2 indicate that bobcat populations in these areas appear to be more isolated from the rest of the state and less likely to mix with nearby populations as ecoregions $3,4,5$, and 6 .

Another important finding of this study was the discovery of the $A \rightarrow G$ mutation at position 4286 of the genome, which was present in 22 / 93 (23.7\%) of the viral sequences examined. As far as we are aware this mutation has not been described previously and may represent a locally evolved type of Carnivore protoparvovirus 1. Another recent study found much greater molecular diversity than was previously expected when examining CPV types worldwide (Li et al. 2017). When completing a diversity study Ohneiser et al. (2015) found CPV variants unique to New Zealand. As such, many local Carnivore protoparvovirus 1 variants are likely on the landscape and have yet to be discovered in locations across the world.

This study also documented three individual animals that were co-infected with different haplotypes of Carnivore protoparvovirus 1. Of these three bobcats, two were infected with different haplotypes of CPV-2 / CPV-2a, which has also been reported in domestic felids (Battilani et al. 2007). However, one bobcat was found to be infected with both CPV-2 / CPV-2a and FPV. Though rare, this co-infection has been documented in domestic cats in Italy (Battilani et al. 2011, 2013). The haplotype that this bobcat was infected with, H_13 of CPV-2 / CPV-2a is an intermediary form between CPV and FPV that was not found in any other animals examined in this study. 


\section{Management Implications}

This study provides the first prevalence rates for Carnivore protoparvovirus 1 infections in bobcats in West Virginia and the Appalachian Mountains of the eastern United States. The viral prevalence hotspot identified by this research should be used as a focal starting point for any planned management actions since this area is presumed to have the most significant impact on West Virginia’s bobcats. Continued monitoring should occur in this region since the virus may be rapidly evolving, as is evidenced by the new haplotype identified in this study. In contrast, many areas in central or southern West Virginia have prevalence rates lower than the rest of the state. It is in these areas that these viruses are probably having a minimal impact; therefore, resources should be focused elsewhere. In conclusion, this study found no evidence of an association between areas of dense human populations and the prevalence of Carnivore protoparvovirus 1 in the wild bobcat population. Additional research is needed to investigate the role that domestic felids play in West Virginia in terms of being a potential viral reservoir.

\section{Acknowledgements}

The research for this section was completed jointly with Stephanie Landry. She contributed the introduction for the final document and completed roughly half of the lab work used to generate these data. The DNA sequencing in this study was completed by the West Virginia University Genomics Core Facility. We thank the hunters and trappers of West Virginia for donating the bobcat carcasses from which spleen samples were collected. We also thank Avery Owers, Brittany Demski, Jarrod Ball for helping with DNA extractions and PCR reactions. Finally, we thank the WVDNR personnel from District 2 that assisted in the bobcat spleen DNA extractions. Financial support for this dissertation was provided by the West 
Virginia Division of Natural Resources through a grant with the U.S. Fish and Wildlife Service Wildlife and Sport Fish Restoration Program (W-48-R) and matching state funds. 


\section{References}

Allison, A., D. Kohler, K. Fox, J. Brown, R. Gerhold, V. Shearn-Bochsler, E. Dubovi, C Parrish, and E Holmes. 2013. Frequent Cross-Species Transmission of Parvoviruses Among Diverse Carnivore Hosts. Journal of Virology 87:2342-2347.

Bandelt H-J., Forster P., Röhl A. (1999) Median-joining Networks for Inferring Intraspecific Phylogenies. Molecular Biology and Evolution 16:37-48.

Battilani, M., A. Balboni, M. Giunti, and S. Prosperi. 2013. Co-infection with Feline and Canine Parvovirus in a Cat. Veterinaria Italiana 49:127-129.

Battilani, M., A. Balboni, M. Ustulin, M. Giunti, A. Scagliarini, and S. Prosperi. 2011. Genetic Complexity and Multiple Infections with more Parvovirus Species in Naturally Infected Cats. Veterinary Research 42:43.

Battilani, M., L. Gallina, F. Vaccari, and L. Morganti. 2007. Co-Infection with Multiple Variants of Canine Parvovirus Type 2 (CPV-2). Veterinary Research Communications; Dordrecht 31:209-12.

Berthier, K., M. Langlais, P. Auger, and D. Pontier. 2000. Dynamics of a Feline Virus with Two Transmission Modes within Exponentially Growing Host Populations. Proceedings of the Royal Society B: Biological Sciences 267:2049-2056.

Buonavoglia, C., V. Martella, A. Pratelli, M. Tempesta, A. Cavalli, D. Buonavoglia, G. Bozzo, G. Elia, N. Decaro, and L. Carmichael. 2001. Evidence for Evolution of Canine Parvovirus Type 2 in Italy. Journal of General Virology 82:3021-3025.

Carver, S., S. N. Bevins, M. R. Lappin, E. E. Boydston, L. M. Lyren, M. Alldredge, K. A. Logan, L. L. Sweanor, S. P. D. Riley, L. E. K. Serieys, R. N. Fisher, T. W. Vickers, W. Boyce, R. McBride, M. C. Cunningham, M. Jennings, J. Lewis, T. Lunn, K. R. Crooks, and S. VandeWoude. 2016. Pathogen Exposure Varies Widely Among Sympatric Populations of Wild and Domestic Felids Across the United States. Ecological Applications 26:367-381.

Clegg, S, K. Coyne, S. Dawson, N. Spibey, R. Gaskell, and A. Radford. 2012. Canine Parvovirus in Asymptomatic Feline Carriers. Veterinary Microbiology 157:78-85.

Cotmore, S. F., M. Agbandje-McKenna, J. A. Chiorini, D. V. Mukha, D. J. Pintel, J. Qiu, M. Soderlund-Venermo, P. Tattersall, P. Tijssen, D. Gatherer, and A. J. Davison. 2014. The family Parvoviridae. Archives of Virology 159:1239-1247.

Csiza, C. K., A. D. Lahunta, F. W. Scott, and J. H. Gillespie. 1971. Pathogenesis of Feline Panleukopenia Virus in Susceptible Newborn Kittens II. Pathology and Immunofluorescence. Infection and Immunity 3:838-846. 
Decaro, N., C. Desario, M. Lucente, F. Amorisco, M. Campolo, G. Elia, A. Cavalli, V. Martella, and C Buonavoglia. 2008. Specific Identification of Feline Panleukopenia Virus and its Rapid Differentiation from Canine Parvoviruses Using Minor Groove Binder Probes. Journal of Virological Methods 147:67-71.

Decaro, N, D. Buonavoglia, C. Desario, F. Amorisco, M. Colaianni, A. Parisi, V. Terio, G. Elia, M. Lucente, A. Cavalli, V. Martella, and C. Buonavoglia. 2010. Characterisation of Canine Parvovirus Strains Isolated from Cats with Feline Panleukopenia. Research in Veterinary Science 89:275-278.

Demeter, Z., E. Palade, and M. Rusvai. 2010. Feline Panleukopenia Virus Infection in Various Species from Hungary. Lucrari Stiintifice Medicina Veterinara 43:73-81.

Desario, C., and N. Decaro. 2005. Canine Parvovirus Infection: Which Diagnostic Test for Virus?. Journal of Virological Methods 126:179-185.

Foley, J., P. Swift, K. Fleer, S. Torres, Y. Girard, and C. Johnson. 2013. Risk Factors for Exposure to Feline Pathogens in California Mountain Lions (Puma concolor). Journal of Wildlife Diseases 49: 279-293.

Getis, A., and J. K. Ord. 1992. The Analysis of Spatial Association by Use of Distance Statistics. Geographical Analysis 24:189-206.

Hall, T.A. 1999. BioEdit: A User-Friendly Biological Sequence Alignment Editor and Analysis Program for Windows 96/98/NT. Nucleic Acids Symposium Series 41:95-98.

Haynes, S., and S. Holloway. 2012. Identification of Parvovirus in the Bone Marrow of Eight Cats. Australian Veterinary Journal 90: 136-139.

Hoelzer, K., L. A. Shackelton, C. R. Parrish, and E. C. Holmes. 2008. Phylogenetic Analysis Reveals the Emergence, Evolution and Dispersal of Carnivore Parvoviruses. Journal of General Virology 89:2280-2289.

Horiuchi, M., K. Yuri, T. Soma, H. Katae, H. Nagasawa, and M. Shinagawa. 1996. Differentiation of Vaccine Virus from Field Isolates of Feline Panleukopenia Virus by Polymerase Chain Reaction and Restriction Fragment Length Polymorphism Analysis. Veterinary Microbiology 53:283-293.

Ikeda, Y., M. Mochizuki, R. Naito, K. Nakamura, T. Miyazawa, T. Mikami, and E. Takahashi. 2000. Predominance of Canine Parvovirus (CPV) in Unvaccinated Cat Populations and Emergence of New Antigenic Types of CPVs in Cats. Virology 278:13-19.

Kruse, B., S. Unterer, K. Horlacher, C. Sauter-Louis, and K. Hartmann. 2010. Prognostic Factors in Cats with Feline Panleukopenia. Journal of Veterinary Internal Medicine 24:12711276. 
Li, G., S. Ji, X. Zhai, Y. Zhang, J. Liu, M. Zhu, J. Zhou, and S. Su. 2017. Evolutionary and Genetic Analysis of the VP2 Gene of Canine Parvovirus. BMC Genomics 18:534.

Meurs, K., P. Fox, A. Magnon, S. Liu, and J. Towbin. 2000. Molecular Screening by Polymerase Chain Reaction Detects Panleukopenia Virus DNA in Formalin-Fixed Hearts from Cats with Idiopathic Cardiomyopathy and Myocarditis. Cardiovascular Pathology 9:119-126.

Ohneiser, S. A., S. F. Hills, N. J. Cave, D. Passmore, and M. Dunowska. 2015. Canine parvoviruses in New Zealand form a monophyletic group distinct from the viruses circulating in other parts of the world. Veterinary Microbiology 178:190-200.

Ord, J. K., and A. Getis. 1995. Local Spatial Autocorrelation Statistics: Distributional Issues and an Application. Geographical Analysis 27:286-306.

Schunck, B., W. Kraft, and U. Truyen. 1995. A Simple Touch-Down Polymerase Chain Reaction for the Detection of Canine Parvovirus and Feline Panleukopenia Virus in Feces. Journal of Virological Methods 55:427-433.

Siegl, G., R. Bates, K. Berns, B. Carter, D. Kelly, E. Kurstak, and P. Tattersall. 1985. Characteristics and Taxonomy of Parvoviridae. Intervirology 23:61-73.

Stuetzer, B., and K. Hartmann. 2014. Feline Parvovirus Infection and Associated Diseases. The Veterinary Journal 201:150-155.

Uhlig, H. G., and H. L. Wilson. 1952. A Method of Evaluating an Annual Mast Index. The Journal of Wildlife Management 16:338-343.

United States Census Bureau, Population Division. 2016. Annual Estimates of the Resident Population: April 1, 2010 to July 1, 2016.

Wasieri, J., G. Schmiedeknecht, C. Forster, M. Konig, and M. Reinacher. 2009. Parvovirus Infection in a Eurasian Lynx (Lynx lynx) and in a European Wildcat (Felis sivestris silvestris). Journal of Comparative Pathology 140:203-20.

Zhou, P., W. Zeng, X. Zhang, and S. Li. 2017. The Genetic Evolution of Canine Parvovirus - A New Perspective. Y. Cao, editor. PLOS ONE 12:e0175035. 


\section{Appendix}

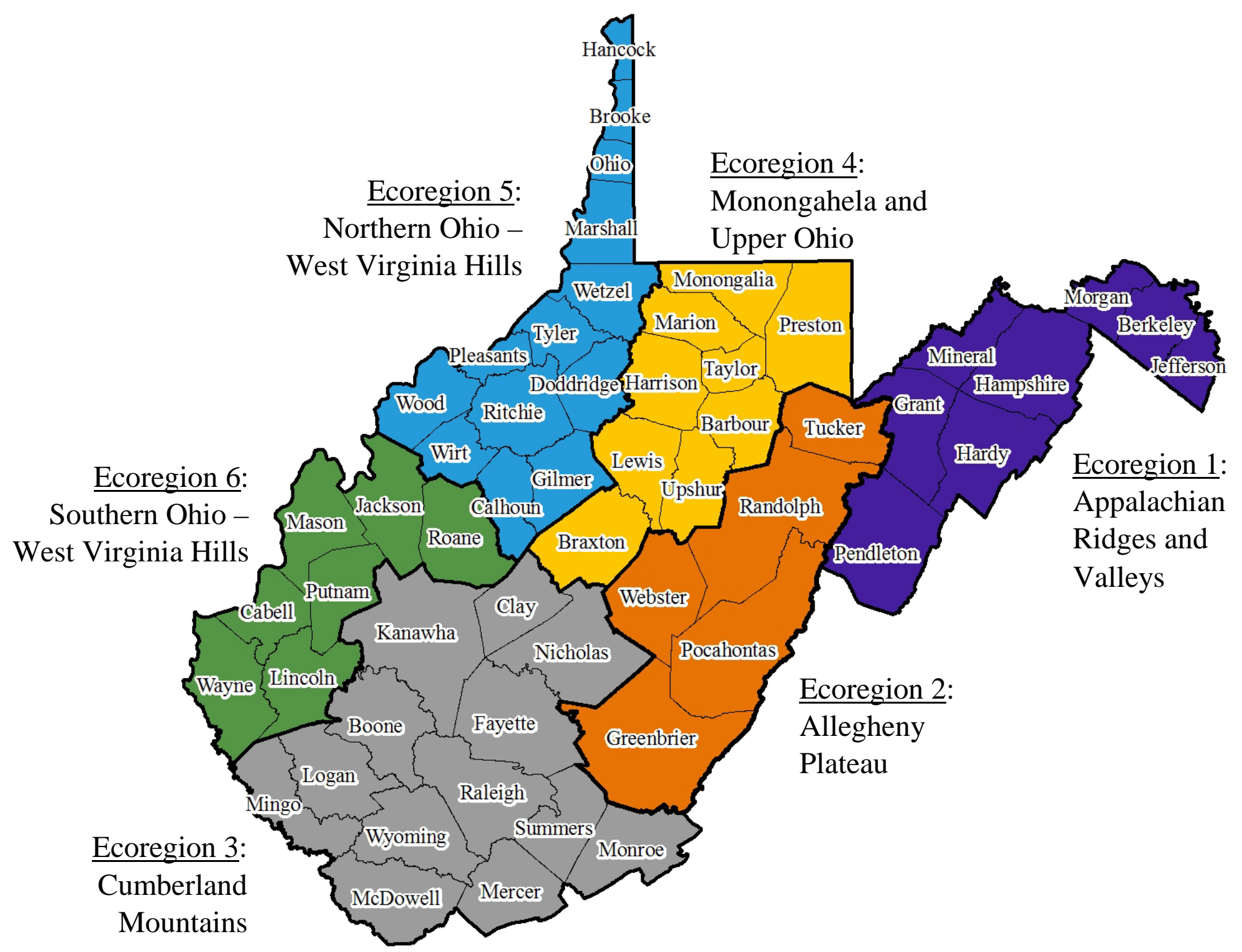

Appendix Figure 1 - Map of the ecological regions of West Virginia, as described by Uhlig and Wilson (1952). 
Appendix Table 1 - For each location sampled during the 2015 - 2016 bobcat hair snaring seasons in West Virginia, the following data are recorded: the grid number, year the grid was sampled, the county sampled, the ecoregion in which this county is located (appendix Figure 1), number of bobcat detections recorded by qPCR analysis, calculated 4week detection probability as estimated by the previously constructed occupancy model (Chapter 3 ) for that particular grid, the minimum known alive (MKA, which is a measurement of the number of unique individuals identified, not a measurement of the census population), the minimum density of bobcats calculated for $100 \mathrm{~km}^{2}$, minimum number of bobcats calculated for $100 \mathrm{~km}^{2}$ when taking the detection probability for that grid into account (100km² DET), and whether or not a location sampled in 2016 was previously sampled in 2016 (Redo).

\begin{tabular}{|c|c|c|c|c|c|c|c|c|c|}
\hline $\begin{array}{l}\text { Sample } \\
\text { Grid }\end{array}$ & Year & County & Ecoregion & Detections & $\begin{array}{c}\text { Detection } \\
\text { Probability }\end{array}$ & MKA & $\begin{array}{c}\text { Min. Density / } \\
100 \mathrm{~km}^{2}\end{array}$ & $\begin{array}{l}\text { Min. Density / } \\
100 \mathrm{~km}^{2} \mathrm{DET}\end{array}$ & Redo \\
\hline $1-1$ & 2015 & Harrison & 4 & 16 & $0.577 \pm 0.065$ & 11 & 4.40 & $7.619 \pm 0.496$ & \\
\hline $1-2$ & 2015 & Wetzel & 5 & 13 & $0.549 \pm 0.066$ & 7 & 2.80 & $5.095 \pm 0.337$ & \\
\hline $1-3$ & 2015 & Monongalia & 4 & 16 & $0.553 \pm 0.065$ & 9 & 3.60 & $6.498 \pm 0.427$ & \\
\hline $1-4$ & 2015 & Tucker & 2 & 14 & $0.550 \pm 0.066$ & 4 & 1.60 & $2.905 \pm 0.192$ & \\
\hline $1-5$ & 2015 & Preston & 4 & 16 & $0.550 \pm 0.066$ & 11 & 4.40 & $7.990 \pm 0.528$ & \\
\hline $2-1$ & 2015 & Hampshire & 1 & 9 & $0.621 \pm 0.071$ & 5 & 2.00 & $3.218 \pm 0.231$ & \\
\hline $2-2$ & 2015 & Morgan & 1 & 4 & $0.549 \pm 0.066$ & 2 & 0.80 & $1.456 \pm 0.096$ & \\
\hline $2-3$ & 2015 & Hardy & 1 & 4 & $0.553 \pm 0.065$ & 3 & 1.20 & $2.167 \pm 0.142$ & \\
\hline $2-4$ & 2015 & Grant & 1 & 16 & $0.605 \pm 0.068$ & 7 & 2.80 & $4.627 \pm 0.315$ & \\
\hline $2-5$ & 2015 & Pendleton & 1 & 8 & $0.563 \pm 0.065$ & 3 & 1.20 & $2.129 \pm 0.138$ & \\
\hline $3-1$ & 2015 & Lewis & 4 & 8 & $0.552 \pm 0.065$ & 1 & 0.40 & $0.724 \pm 0.047$ & \\
\hline $3-2$ & 2015 & Braxton & 4 & 23 & $0.553 \pm 0.065$ & 21 & 8.40 & $15.18 \pm 1.000$ & \\
\hline $3-3$ & 2015 & Randolph & 2 & 17 & $0.553 \pm 0.065$ & 6 & 2.40 & $4.336 \pm 0.285$ & \\
\hline $3-4$ & 2015 & Pocahontas & 2 & 9 & $0.568 \pm 0.065$ & 6 & 2.40 & $4.221 \pm 0.274$ & \\
\hline $3-5$ & 2015 & Webster & 2 & 1 & $0.622 \pm 0.072$ & 0 & 0 & 0 & \\
\hline $4-1$ & 2015 & Fayette & 3 & 3 & $0.550 \pm 0.066$ & 0 & 0 & 0 & \\
\hline $4-2$ & 2015 & Summers & 3 & 16 & $0.570 \pm 0.065$ & 8 & 3.20 & $5.610 \pm 0.365$ & \\
\hline $4-3$ & 2015 & Greenbrier & 2 & 3 & $0.548 \pm 0.066$ & 1 & 0.40 & $0.728 \pm 0.048$ & \\
\hline $4-4$ & 2015 & Monroe & 3 & 3 & $0.554 \pm 0.065$ & 1 & 0.40 & $0.721 \pm 0.047$ & \\
\hline $4-5$ & 2015 & Raleigh & 3 & 4 & $0.551 \pm 0.066$ & 2 & 0.80 & $1.450 \pm 0.095$ & \\
\hline $5-1$ & 2015 & Boone & 3 & 24 & $0.549 \pm 0.066$ & 16 & 6.40 & $11.63 \pm 0.770$ & \\
\hline $5-2$ & 2015 & Lincoln & 6 & 16 & $0.548 \pm 0.066$ & 9 & 3.60 & $6.564 \pm 0.435$ & \\
\hline $5-3$ & 2015 & Mason & 6 & 1 & $0.561 \pm 0.065$ & 1 & 0.40 & $0.712 \pm 0.046$ & \\
\hline $5-4$ & 2015 & Wayne & 6 & 11 & $0.579 \pm 0.065$ & 3 & 1.20 & $2.071 \pm 0.135$ & \\
\hline $5-5$ & 2015 & Putnam & 6 & 6 & $0.550 \pm 0.066$ & 3 & 1.20 & $2.180 \pm 0.144$ & \\
\hline $6-1$ & 2015 & Wirt & 5 & 0 & $0.609 \pm 0.069$ & 0 & 0 & 0 & \\
\hline $6-2$ & 2015 & Ritchie & 5 & 9 & $0.553 \pm 0.065$ & 5 & 2.00 & $3.611 \pm 0.237$ & \\
\hline
\end{tabular}




\begin{tabular}{|c|c|c|c|c|c|c|c|c|c|}
\hline $6-3$ & 2015 & Doddridge & 5 & 3 & $0.552 \pm 0.065$ & 2 & 0.80 & $1.447 \pm 0.095$ & \\
\hline $6-4$ & 2015 & Roane & 6 & 1 & $0.557 \pm 0.065$ & 1 & 0.40 & $0.716 \pm 0.046$ & \\
\hline $6-5$ & 2015 & Calhoun & 5 & 4 & $0.559 \pm 0.065$ & 2 & 0.80 & $1.430 \pm 0.093$ & \\
\hline $\mathrm{x} 1-1$ & 2016 & Preston & 5 & 3 & $0.235 \pm 0.036$ & 1 & 0.40 & $1.696 \pm 0.061$ & \\
\hline $\mathrm{x} 1-2$ & 2016 & Tucker & 4 & 4 & $0.125 \pm 0.032$ & 0 & 0 & 0 & YES \\
\hline $\mathrm{x} 1-3$ & 2016 & Barbour & 4 & 3 & $0.237 \pm 0.036$ & 3 & 1.20 & $5.053 \pm 0.183$ & \\
\hline $\mathrm{x} 1-4$ & 2016 & Marion & 4 & 2 & $0.241 \pm 0.036$ & 2 & 0.80 & $3.308 \pm 0.121$ & \\
\hline $\mathrm{x} 1-5$ & 2016 & Marshall & 5 & 5 & $0.238 \pm 0.036$ & 3 & 1.20 & $5.034 \pm 0.183$ & \\
\hline$x 2-1$ & 2016 & Pendleton & 1 & 10 & $0.298 \pm 0.056$ & 3 & 1.20 & $4.020 \pm 0.229$ & \\
\hline$x 2-2$ & 2016 & Grant & 1 & 4 & $0.285 \pm 0.050$ & 2 & 0.80 & $2.799 \pm 0.141$ & \\
\hline$x 2-3$ & 2016 & Hardy & 1 & 7 & $0.253 \pm 0.038$ & 8 & 3.20 & $12.64 \pm 0.487$ & \\
\hline$x 2-4$ & 2016 & Mineral & 1 & 0 & $0.235 \pm 0.036$ & 0 & 0 & 0 & \\
\hline$x 2-5$ & 2016 & Hampshire & 1 & 1 & $0.235 \pm 0.036$ & 1 & 0.40 & $1.698 \pm 0.061$ & \\
\hline x3-1 & 2016 & Clay & 3 & 2 & $0.235 \pm 0.036$ & 2 & 0.80 & $3.403 \pm 0.123$ & \\
\hline x3-2 & 2016 & Upshur & 4 & 10 & $0.233 \pm 0.036$ & 3 & 1.20 & $5.131 \pm 0.185$ & \\
\hline x3-3 & 2016 & Randolph & 2 & 8 & $0.231 \pm 0.036$ & 5 & 2.00 & $8.627 \pm 0.311$ & \\
\hline x3-4 & 2016 & Randolph & 2 & 1 & $0.125 \pm 0.032$ & 1 & 0.40 & $3.181 \pm 0.102$ & YES \\
\hline x3-5 & 2016 & Pocahontas & 2 & 1 & $0.230 \pm 0.036$ & 1 & 0.40 & $1.738 \pm 0.062$ & \\
\hline$x 4-1$ & 2016 & Greenbrier & 2 & 8 & $0.233 \pm 0.036$ & 4 & 1.60 & $6.865 \pm 0.247$ & \\
\hline$x 4-2$ & 2016 & Greenbrier & 2 & 2 & $0.124 \pm 0.031$ & 2 & 0.80 & $6.434 \pm 0.205$ & YES \\
\hline$x 4-3$ & 2016 & Monroe & 3 & 6 & $0.247 \pm 0.037$ & 2 & 0.80 & $3.226 \pm 0.121$ & \\
\hline$x 4-4$ & 2016 & Raleigh & 3 & 2 & $0.125 \pm 0.032$ & 2 & 0.80 & $6.391 \pm 0.204$ & YES \\
\hline$x 4-5$ & 2016 & Fayette & 6 & 1 & $0.125 \pm 0.032$ & 0 & 0 & 0 & YES \\
\hline x5-1 & 2016 & Putnam & 6 & 2 & $0.124 \pm 0.031$ & 2 & 0.80 & $6.404 \pm 0.204$ & YES \\
\hline$x 5-2$ & 2016 & Mason & 6 & 1 & $0.127 \pm 0.032$ & 1 & 0.40 & $3.130 \pm 0.101$ & YES \\
\hline$x 5-3$ & 2016 & Wayne & 6 & 0 & $0.240 \pm 0.036$ & 0 & 0.00 & 0 & \\
\hline$x 5-4$ & 2016 & Kanawha & 3 & 1 & $0.231 \pm 0.036$ & 1 & 0.40 & $1.731 \pm 0.062$ & \\
\hline$x 5-5$ & 2016 & Boone & 3 & 1 & $0.124 \pm 0.031$ & 2 & 0.80 & $6.427 \pm 0.205$ & YES \\
\hline x6-1 & 2016 & Wood & 5 & 5 & $0.127 \pm 0.032$ & 5 & 2.00 & $15.72 \pm 0.508$ & YES \\
\hline x6-2 & 2016 & Gilmer & 5 & 5 & $0.244 \pm 0.037$ & 2 & 0.80 & $3.265 \pm 0.121$ & \\
\hline x6-3 & 2016 & Tyler & 5 & 2 & $0.231 \pm 0.036$ & 0 & 0 & 0 & \\
\hline$x 6-4$ & 2016 & Pleasants & 5 & 1 & $0.230 \pm 0.036$ & 1 & 0.40 & $1.733 \pm 0.062$ & \\
\hline$x 6-5$ & 2016 & Jackson & 6 & 2 & $0.230 \pm 0.036$ & 2 & 0.80 & $3.472 \pm 0.125$ & \\
\hline
\end{tabular}


Appendix Table 2 - A detailed account of the number of bobcat spleen samples tested from each participating West Virginia county and the findings of the tests. Spleen samples were collected from bobcats harvested between 2014 2016 and samples confirmed as testing positive produced a DNA sequence consistent with that of Carnivore protoparvovirus 1.

\begin{tabular}{|c|c|c|c|c|c|c|c|}
\hline County & Total Tested & $\begin{array}{c}\text { Tested } \\
\text { Negative }\end{array}$ & $\begin{array}{c}\text { Tested } \\
\text { Positive }\end{array}$ & $\begin{array}{l}\text { CPV-2 / } \\
\text { CPV-2a }\end{array}$ & $\mathrm{CPV}-2 \mathrm{~b}$ & CPV-2c & FPV \\
\hline Barbour & 11 & 9 & 2 & 2 & 0 & 0 & 0 \\
\hline Boone & 11 & 10 & 1 & 1 & 0 & 0 & 0 \\
\hline Braxton & 9 & 9 & 0 & 0 & 0 & 0 & 0 \\
\hline Brooke & 2 & 2 & 0 & 0 & 0 & 0 & 0 \\
\hline Calhoun & 3 & 1 & 2 & 2 & 0 & 0 & 0 \\
\hline Clay & 4 & 4 & 0 & 0 & 0 & 0 & 0 \\
\hline Doddridge & 9 & 4 & 5 & 5 & 0 & 0 & 0 \\
\hline Fayette & 11 & 8 & 3 & 2 & 1 & 0 & 0 \\
\hline Gilmer & 16 & 11 & 5 & 4 & 1 & 0 & 0 \\
\hline Grant & 10 & 9 & 1 & 1 & 0 & 0 & 0 \\
\hline Greenbrier & 4 & 4 & 0 & 0 & 0 & 0 & 0 \\
\hline Hampshire & 7 & 5 & 2 & 1 & 0 & 0 & 1 \\
\hline Hardy & 5 & 4 & 1 & 1 & 0 & 0 & 0 \\
\hline Harrison & 9 & 6 & 3 & 2 & 1 & 0 & 0 \\
\hline Jackson & 4 & 2 & 2 & 2 & 0 & 0 & 0 \\
\hline Kanawha & 34 & 32 & 2 & 1 & 0 & 0 & 1 \\
\hline Lewis & 12 & 7 & 5 & 5 & 0 & 0 & 0 \\
\hline Lincoln & 23 & 22 & 1 & 0 & 1 & 0 & 0 \\
\hline Logan & 22 & 21 & 1 & 1 & 0 & 0 & 0 \\
\hline Marion & 8 & 6 & 2 & 2 & 0 & 0 & 0 \\
\hline Marshall & 11 & 5 & 6 & 5 & 1 & 0 & 0 \\
\hline Mason & 11 & 4 & 7 & 6 & 0 & 0 & 1 \\
\hline McDowell & 2 & 2 & 0 & 0 & 0 & 0 & 0 \\
\hline Mercer & 12 & 11 & 1 & 1 & 0 & 0 & 0 \\
\hline Mineral & 4 & 4 & 0 & 0 & 0 & 0 & 0 \\
\hline Monongalia & 8 & 6 & 2 & 1 & 1 & 0 & 0 \\
\hline Monroe & 4 & 3 & 1 & 1 & 0 & 0 & 0 \\
\hline Nicholas & 21 & 18 & 3 & 3 & 0 & 0 & 0 \\
\hline Pendleton & 15 & 13 & 2 & 2 & 0 & 0 & 0 \\
\hline Pleasants & 2 & 1 & 1 & 1 & 0 & 0 & 0 \\
\hline Pocahontas & 9 & 8 & 1 & 1 & 0 & 0 & 0 \\
\hline Preston & 18 & 16 & 2 & 1 & 1 & 0 & 0 \\
\hline Putnam & 12 & 11 & 1 & 1 & 0 & 0 & 0 \\
\hline Raleigh & 35 & 32 & 3 & 2 & 1 & 0 & 0 \\
\hline Randolph & 26 & 22 & 4 & 2 & 2 & 0 & 0 \\
\hline Ritchie & 24 & 21 & 3 & 3 & 0 & 0 & 0 \\
\hline
\end{tabular}




\begin{tabular}{cccccccc} 
Roane & 13 & 8 & 5 & 5 & 0 & 0 & 0 \\
Summers & 10 & 7 & 3 & 3 & 0 & 0 & 0 \\
Taylor & 1 & 1 & 0 & 0 & 0 & 0 & 0 \\
Tucker & 3 & 2 & 1 & 1 & 0 & 0 & 0 \\
Tyler & 6 & 5 & 1 & 0 & 1 & 0 & 0 \\
Wayne & 2 & 2 & 0 & 0 & 0 & 0 & 0 \\
Webster & 13 & 13 & 0 & 0 & 0 & 0 & 0 \\
Wetzel & 33 & 21 & 12 & 12 & 0 & 0 & 0 \\
Wirt & 10 & 9 & 1 & 1 & 0 & 0 & 0 \\
Wood & 4 & 4 & 0 & 0 & 0 & 0 & 0 \\
\hline
\end{tabular}

Appendix Table 3 - This table contains the raw data used to create the proportion charts on the Carnivore protoparvovirus 1 haplotype network constructed in this study (Figure 17) for DNA sequences isolated from bobcat spleens collected in West Virginia between 2014 - 2016. A map of the ecological regions of West Virginia is included in this Appendix as figure 1.

West Virginia Ecological Region

\begin{tabular}{cllllll}
\hline Haplotype ID & 1 & 2 & 3 & 4 & 5 & 6 \\
\hline H_1 & 0 & 0 & 2 & 5 & 11 & 1 \\
H_2 & 0 & 0 & 1 & 1 & 1 & 1 \\
H_3 & 0 & 0 & 0 & 1 & 1 & 0 \\
H_4 & 0 & 1 & 1 & 4 & 5 & 2 \\
H_5 & 0 & 1 & 4 & 2 & 13 & 10 \\
H_6 & 1 & 1 & 5 & 0 & 1 & 0 \\
H_7 & 0 & 1 & 2 & 0 & 0 & 0 \\
H_8 & 0 & 0 & 0 & 0 & 0 & 1 \\
H_9 & 0 & 0 & 0 & 2 & 1 & 0 \\
H_10 & 0 & 0 & 0 & 1 & 0 & 0 \\
H_11 & 0 & 0 & 1 & 0 & 0 & 0 \\
H_12 & 2 & 0 & 0 & 0 & 0 & 0 \\
H_13 & 1 & 0 & 0 & 0 & 0 & 0 \\
H_14 & 1 & 0 & 0 & 0 & 0 & 0 \\
H_15 & 0 & 0 & 0 & 0 & 1 & 0 \\
H_16 & 1 & 0 & 0 & 0 & 0 & 0 \\
H_17 & 0 & 0 & 1 & 0 & 0 & 0 \\
H_18 & 0 & 0 & 1 & 0 & 0 & 0 \\
Total & 5 & 4 & 9 & 7 & 8 & 5 \\
\hline
\end{tabular}




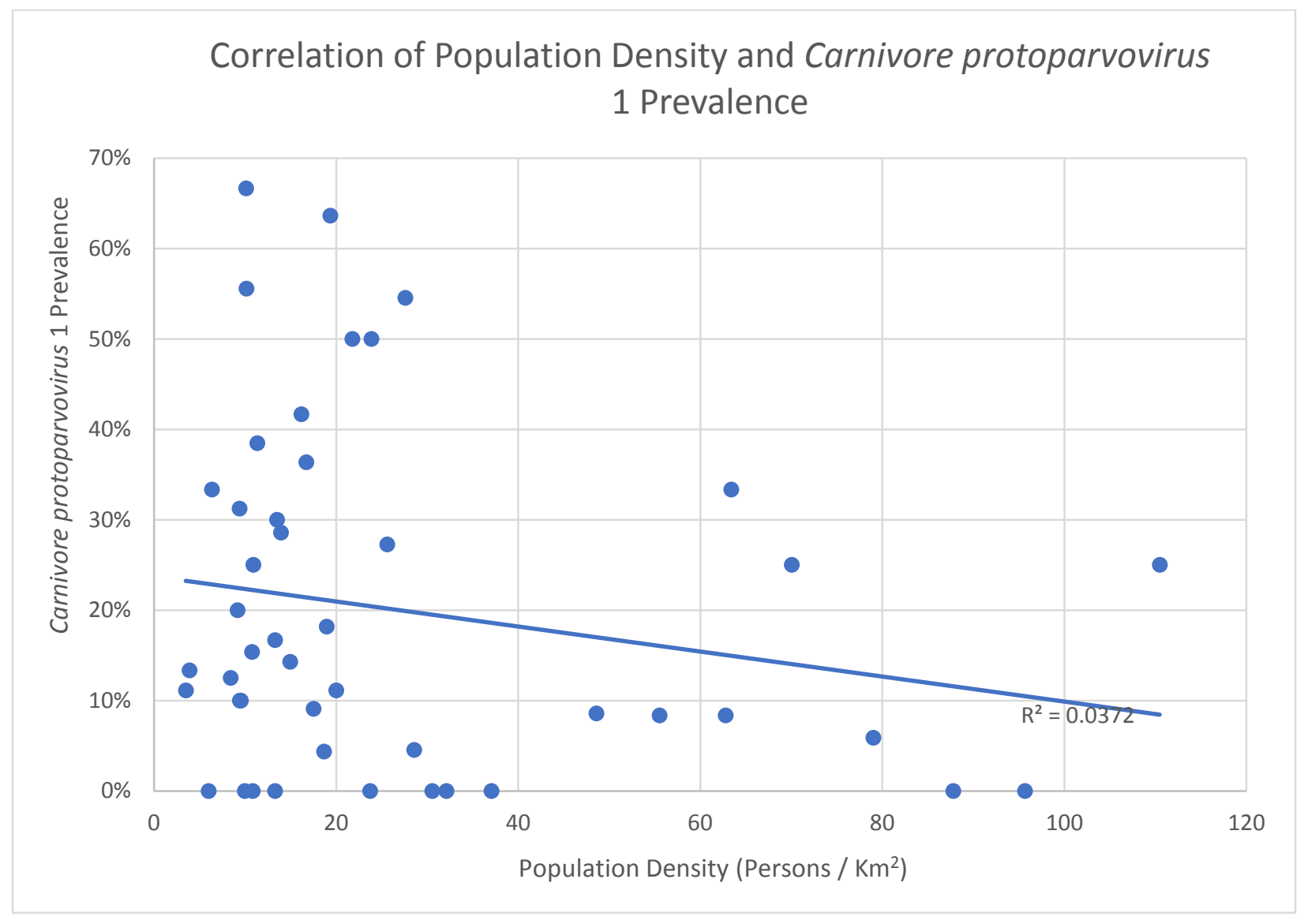

Appendix Figure 2 - Graph of the correlation between the human population density of each West Virginia county from which the prevalence of Carnivore protoparvovirus 1 was calculated. Prevalence rates were determined as the number of bobcat spleens collected from 2014 - 2016 from which Carnivore protoparvovirus 1 DNA was successfully sequenced as compared to all samples tested. 
Date: Oct 23, 2014

\section{IACUC PROTOCOL APPROVAL NOTICE TO THE INVESTIGATOR}

\begin{tabular}{l|l|l|} 
Investigator: James Anderson & ACUC Protocol \#: $14-1108$ \\
Project Title: & Population and landscape genetics of the bobcats (Lynx rufus) of West Virginia
\end{tabular}

Type of approval: New Protocol

Replacing protocol \#:

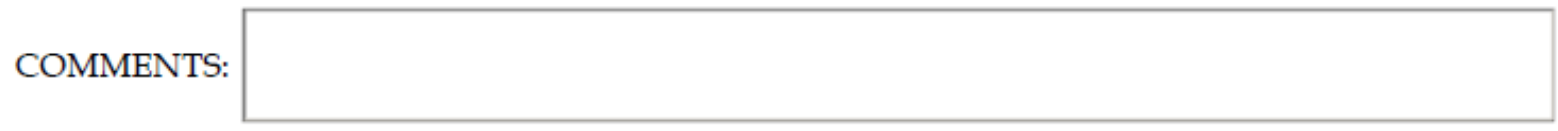

Funding Agency: WVDNR

The West Virginia University Animal Care and Use Committee (WVU ACUC) has granted approval for the above referenced project. Approval was based on review of the protocol and, if appropriate, an inspection of the laboratory/facility space where procedures on animals are done. Should any modifications (including, but not limited to, an increase in the number of animals, changes in procedures, or additions/removals of personnel) be necessary, you must obtain prior approval from the WVU ACUC by submitting an amendment.

NOTE: Please remember that a copy of the complete approved protocol, which will include any amendments, must be posted in the laboratory and be made available to all study personnel.

Initial Approval Date: Oct 23, 2014
Number of animals: undetermined
Scquisition of your animals MUST be through
OLAR
USDA Pain Category: $B \quad$ bubcat

Animals will be taken out of the vivarium and may or may not return. Therefore, this protocol will be monitored for re-approval every 6 months and you will be contacted by members of the WVU ACUC for a laboratory inspection every 6 months. This inspection must be completed prior to the 6 month deadline or your protocol will be closed.

Animals are not taken out of the vivarium OR this is an agricultural or wildlife protocol. Therefore, this protocol - will be reviewed annually for continuing approval. You will receive a blue self inspection form 3 months prior to the 12 month deadline. This form MUST be returned by the date indicated or the protocol will be closed.

ALL personnel on WVU ACUC protocols MUST be up to date with CITI training (good for 3 years) and the Animal Health Questionnaire (good for 1 year) before protocols can be reviewed and/or approved. For assistance with this training, please go to http://oric.research.wvu.edu.

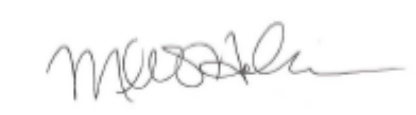

Mindy Hollander

Animal Compliance and Training Officer, Office of Research Integrity and Compliance email: mhollander@mail.wvu.edu phone: 304-293-9368 cell: 304-216-1641

\footnotetext{
Chestnut Ridge Research Building

hone: 304-293-7073 886 Chestnut Ridge Road, Suite 211

\begin{tabular}{l|l} 
Fax: 304-293-3098 & PO Box 6845
\end{tabular}

http://oric.research.wvu.edu/ Morgantown, WV 26506-6845
}

Equal Opportunity/Affirmative Action Institution

Appendix Document 1 - Copy of IACUC Protocol 14-1108 approval form to begin bobcat hair snare trapping. 